\title{
Ab-initio Studies of X-ray Scattering
}

\author{
Dissertation zur Erlangung \\ des mathematisch-naturiwssenschaftlichen Doktorgrades \\ "Doctor rerum naturalium" \\ der Georg-August-Universität Göttingen
}

vorgelegt von

\section{Andrea Debnárová}

aus Banská Bystrica, Slowakei

Göttingen, 2009 
Referent: Prof. Dr. Markus Münzenberg Koreferent: Prof. Dr. Jürgen Troe

Tag der mündlichen Prüfung: 28.08.2009 
Dedicated to the memory of my dear father Ján Debnár.

The sudden loss of him greatly saddened us. 



\section{Preface}

I would like to give many thanks to a number of people, who all in their own way contributed to the making of this thesis. First of all I am very grateful to Prof. Markus Münzenberg for his willingness to take over the position of the primary referee for my dissertation and all his help, and Prof. Dr. Jürgen Troe for taking over the position of the second referee. Additionally I want to thank Prof. Troe for enabling me to perform my PhD work at the Max Planck Institute for Biophysical Chemistry.

A particular thank you is addressed to Inge Dreger and Martin Fechner for the administrative and technical support. Support by the Max-Planck-Society and the SFB755 is gratefully acknowledged.

Gratitude also goes to my colleagues Wilson Quevedo, Jörg Hallman and Gerhard Busse, who not only supported my work by constructive discussion but also created a nice working atmosphere. My supervisor Dr. Simone Techert gets the final big thank you, not only for accepting me into her group at MPI but also for her endless patience, optimism and knowledge. Thank you!

Andrea Debnarova

Göttingen, July 2009 



\section{Contents}

$\begin{array}{lll}1 & \text { Introduction } & 1\end{array}$

\begin{tabular}{lll}
\hline 2 & Theory & 7
\end{tabular}

$2.1 \quad$ Wave Functions and Electron Density $\ldots \ldots \ldots \ldots$

2.1 .1 Born-Oppenheimer Approximation . . . . . . . . . . 8

2.1 .2 Wave Functions of the Nuclei . . . . . . . . . . . . 10

$2.1 .3 \quad$ Electron Wave Functions and Electron Density . . . . . . . 12

$2.2 \quad$ X-Ray Scattering . . . . . . . . . . . . . . . . . . . . . . 17

2.2 .1 Time-Resolved X-Ray Scattering . . . . . . . . . . . . . 17

2.2 .2 Electron Density and its Fourier Transformation . . . . . 20

$2.2 .3 \quad$ Ensemble of Molecules, Gases and Alignment . . . . . . . . 24

2.3 Density Functional Theory $\ldots \ldots \ldots \ldots \ldots$

$2.3 .1 \quad$ Density Functional Theory $\ldots \ldots \ldots \ldots$. . . . . . . 27

$2.3 .2 \quad$ Time-Dependent Density Functional Theory . . . . . . . . . 32

$2.3 .3 \quad$ Strong Fields: TDDFT Beyond Linear Response . . . . . . 34

2.4 Nuclear Wavepacket Dynamics $\ldots \ldots \ldots \ldots$

$3 \quad$ X-ray Scattering and Photoisomerization of Stilbene 39

3.1 Introduction . . . . . . . . . . . . . . . . . . . . . . . 39

3.2 Potential Energy Surface $\ldots \ldots \ldots \ldots$. . . . . . . . . . . 45

3.3 The X-Ray Scattering Spectra $\ldots \ldots \ldots \ldots \ldots$. . . . . . . 47

3.4 Dynamics $\ldots \ldots \ldots \ldots \ldots$

$3.5 \quad$ Conclusions $\ldots \ldots \ldots \ldots \ldots \ldots$

4 X-ray Scattering and Photodissociation of Iodine 61

4.1 Introduction . . . . . . . . . . . . . . . . . . . . . . . 61 
vi

4.2 The $\mathrm{I}_{2}$ X-Ray Scattering Spectra and Excited States . . . . . . . . 65

4.3 Observing $\mathrm{I}_{2}$ Dissociation with Time-Resolved X-Ray Scattering . 71

4.4 Conclusions $\ldots \ldots \ldots \ldots \ldots \ldots$

\begin{tabular}{|lll}
5 X-ray Scattering on Aligned Molecules & 81
\end{tabular}

5.1 Introduction . . . . . . . . . . . . . . . . . . . . 81

$5.2 \quad$ X-ray Scattering on Aligned Systems $\ldots \ldots \ldots \ldots$. . . . . . . 84

5.3 Time-Resolved Scattering on Aligned Systems . . . . . . . . . . . 94

5.4 Conclusions . . . . . . . . . . . . . . . . . . . . . . . . . . 104

$\begin{array}{lll}6 & \text { Water in Strong Soft X-Ray Laser Fields } & 107\end{array}$

$6.1 \quad$ Introduction . . . . . . . . . . . . . . . . . . . . . 107

6.2 Computational Details . . . . . . . . . . . . . . . . . . . 112

6.3 Energy Absorption . . . . . . . . . . . . . . . . . . . 115

6.4 Distances and Velocities . . . . . . . . . . . . . . . . 124

$6.5 \quad$ Electron Density Change. . . . . . . . . . . . . . . . . . . 130

6.6 Conclusions . . . . . . . . . . . . . . . . . . . . . . . . 140

\begin{tabular}{lll}
\hline 7 & Summary & 143
\end{tabular}

\begin{tabular}{|l|l|}
\hline A Program for x-ray scattering on aligned molecules & 159
\end{tabular} 


\section{Chapter 1}

\section{Introduction}

For many decades, x-ray crystallography has dominated the field of atomic scale structural measurements. Whenever crystallization of a system is possible it has been an excellent method, achieving atomic resolution even for complicated systems like large biomolecules such as proteins. It has not been constrained to static structure determination, but has also been used to determine the structure of reaction intermediates, for example an early intermediate of bacteriorhodopsin photocycle trapped at low temperature ${ }^{1}$. Even pump-probe time-resolved experiments have been devised and performed studying a variety of systems, such as picosecond excited state structural change in organic crystals of 4,4'-dimethylaminobenzonitrile ${ }^{2}$, or Ångstrom scale atomic motion involved in nonthermal melting of germanium ${ }^{3}$.

However, many important systems, especially in structural biology, cannot be crystallized. Diffraction on these samples does not benefit from Bragg amplification. In the case of crystalline samples the amplification of the signal at the Bragg peaks is caused by the constructive interference from periodically repeated unit cells. Therefore the diffraction signal from noncrystalline samples is much weaker, the increase of the source brightness being the only possibility for increased signal. Until now even the brightest x-ray sources, such as the synchrotrons of the third generation or plasma sources, did not provide enough photons to enable measurable high resolution diffraction on noncrystalline samples.

In recent years the next generation of x-ray sources - free electron lasers (FELs) - have been under construction. They are expected to provide source brilliance on the level where coherent diffraction measurements of noncrystalline 
samples would be possible. However, with such high brilliance radiation damage of the samples becomes an important issue. Using todays synchrotron sources and minimizing the radiation damage by cryoprotection techniques, a beam brightness of 200 photons per $\AA^{2}$ with $1 \AA$ x-rays ${ }^{4}$ is considered the maximum before the radiation damage becomes too strong for successful structural measurement.

Much more intense probe pulses would be necessary for single molecule measurements. At the necessary intensity the effect of the so called Coulomb explosion is unavoidable. The high number of ionization events under the necessary photon fluxes would strip the sample of electrons leaving behind what is in essence a cluster of repulsive positively charged ions. Depending on the size of the original molecule this would disintegrate in time-scales on the order of tens to hundreds femtoseconds. However, it has been reported ${ }^{5}$ that even such intense ultrashort pulses might be used, if they are short enough to provide the coherent diffraction signal in the very first stages of Coulomb explosion, when the structure factor changes of the atoms in the sample caused by photoionization are small enough for reliable structural measurement. The spacial coherence of the FEL pulses opens the door for new imaging techniques such as coherent diffractive imaging. The object is computationally reconstructed from coherent scattering patterns, making use of phase retrieval techniques. $\frac{6778}{67}$ The strong laser fields of FEL provide new challenges for experiments as well as theory. This work is is focused on the detailed description of processes in the sample and the effects they have on x-ray scattering.

As the emergence of the FEL sources opens up new opportunities in material research, the theory and modeling of the behavior of matter in strong x-ray laser fields, as well as novel approaches to scattering, become of an increased importance. The high intensity allows for measurements with increased electron density details refinement due to possible increase of signal to noise ration in the diffuse spectra region. Another topic of interest for theoretical study is the coherent diffraction during the event of Coulomb explosion. Time-resolved x-ray scattering studies of small molecular systems using the strong FEL pulses can elucidate a number of questions concerning their reaction dynamics. This has been so far studied mainly by spectroscopic techniques, which in many cases do not lead to conclusive results.

There is a number of possible theoretical approaches in theoretical studies of molecular structure and dynamics and matter-laser interaction. In the case of 
large molecular systems and clusters (as for example in the work by Neutze et al [5]) the preferred theoretical description is one based on molecular dynamics and stochastic methods. If, however, the studied molecular system is relatively small, bearing in mind the required computational time, a choice of a suitable ab-initio quantum chemistry method is beneficial. This provides a detailed account of quantum mechanical processes involved in the time-dependent changes of the molecular structure in pump-probe experiment, as well as the matter-laser interaction with a specific laser probe pulse. One of the most widely used ab-initio methods for the description of ground state structures is the so called density functional theory (DFT), extended as time-dependent density functional theory (TDDFT) in the case of excited states. Throughout this thesis these methods are not only used in modeling the molecular structure and dynamics but also the matter-laser interaction. Chapter 2 contains the overview of these methods as well as other theoretical and modeling tools used in this thesis.

Focusing on small molecular systems a number of important effects can be studied, benefiting from the improved scattering resolution offered by the strong FEL pulses. Effects such as delocalized electron density distribution, as it is exhibited for example in chemical bonds, can become measurable even in gaseous or liquid samples. A study of the effects of detailed electron density distribution on photoisomerization of stilbene are described in detail in the Chapter 3 .

Another interesting subject for time-resolved studies is the dynamics of chemical reactions. One of widely studied prototypical reactions is the photodissociation and recombination of molecular iodine. The high number of electrons in iodine means a strong scattering signal, so that even an experiment with an $\mathrm{I}_{2}$ gas is possible. The Chapter 4 deals with various aspects of the reaction dynamics, such as coherent and non-coherent processes, periodic features of structural relaxation versus non-periodic dissociative process, as well as small electron density changes caused by electronic excitation, all with respect to x-ray scattering.

Recent advances in laser alignment of molecular systems offer an alternative to proposed single molecule coherent diffraction. Alignment introduces a form of periodicity in the molecular system and so increases the diffraction intensity. As explained in Chapter 5 this enhances the structural information encoded in the scattering images and enables improved resolution in studies of reaction dynamics, as it is shown on the example of the photoisomerization of stilbene. Interesting 
applications could follow also from the fact that the scattering signal is strongly influenced by the alignment axis, which can be modified by attaching suitable polarizable groups onto the studied molecule.

Theoretical studies of Coulomb explosion have gained importance with respect to the interest in the single molecule coherent diffraction. Instead of the usual approach using classical molecular dynamics, the TDDFT approach to the problem is the subject of Chapter 6. This is a computationally expensive method and therefore the size of the studied cluster has to be correspondingly small. However, it has an important advantage in comparison to the stochastic methods as it accounts for the laser field explicitly and so does not impose constrains on the interaction of the laser pulse with the cluster. 


\section{Chapter 2}

\section{Theory}

\subsection{Wave Functions and Electron Density}

Atomic systems, be it molecules, crystals or even just isolated atoms, are in quantum mechanics described by a number of properties. Some of them have a classical equivalent which assists our intuitive understanding of them, forming a bridge between the classical and quantum mechanical world. Others are more or less symbolic, like bonds and molecular orbitals, understanding of which is predominantly based on their theoretical representation by equations and mathematical expressions. Electron density is one of the properties belonging to the first group.

This Chapter is concerned with the understanding of the relationship and interplay between electronic and vibrational wave functions and consequently between the electron densities and vibrational degrees of freedom in a molecule. This understanding is essential for detailed study of x-ray scattering intensities of molecular systems (see Chapter 2.2. A detailed overview can be found in e.g. Ref. [9] and for the detailed evaluation of the vibrational degrees of freedom Ref. [10] or [11.

\subsubsection{Born-Oppenheimer Approximation}

When interested in the physical behavior of a molecular system, the first step in a quantum mechanical study is describing the system through the Schrödinger equation

$$
\hat{H} \Phi=E \Phi
$$


The Hamiltonian of a molecular system is built from a few important terms

$$
\begin{aligned}
& \hat{H}=\hat{T}_{n}+\hat{T}_{e}+\hat{V}_{n e}+\hat{V}_{e e}+\hat{V}_{n n}= \\
& -\sum_{a} \frac{\hbar^{2}}{2 M_{a}} \nabla_{a}^{2}-\sum_{j} \frac{\hbar^{2}}{2 m_{e}} \nabla_{j}^{2} \\
& -\sum_{a} \sum_{j} \frac{Z_{a} e^{2}}{\left|\mathbf{r}_{j}-\mathbf{R}_{a}\right|}+\sum_{i} \sum_{j>i} \frac{e^{2}}{\left|\mathbf{r}_{i}-\mathbf{r}_{j}\right|}+\sum_{a} \sum_{b>a} \frac{Z_{a} Z_{b} e^{2}}{\left|\mathbf{R}_{a}-\mathbf{R}_{b}\right|}
\end{aligned}
$$

where $a, b$ denote nuclei and $i, j$ electron term, thus $\mathbf{R}_{a}, \mathbf{r}_{i}$ correspond to the position of the nuclei and electrons respectively, $M_{a}, m_{e}$ to the mass of nuclei and electrons. In the kinetic energies $\hat{T}$ and particle pair interaction potentials $\hat{V}$ the subscripts $e$ and $n$ denote the dependence of electron and nuclear positions, with for example $\hat{T}_{e}$ being the kinetic energy of the electrons and $\hat{V}_{n e}$ the nuclei-electrons pair interaction potential.

Since the Schrödinger equation of the many particle system 2.2 is a complicated second order partial differential equation, one has to perform a number of approximations in order to solve it. Let us assume that we have the complete set of orthonormal eigenstates $\Psi_{i}(\mathbf{R}, \mathbf{r})$ to the electronic part of the Hamiltonian 2.2 , $\hat{H}_{e}=\hat{T}_{e}+\hat{V}_{n e}+\hat{V}_{e e}+\hat{V}_{n n}$, with the corresponding energy eigenvalues $\mathcal{E}_{i}(\mathbf{R})$. The eigenstates of the total Hamiltonian can be generally written in the form

$$
\Phi(\mathbf{R}, \mathbf{r})=\sum_{i=1}^{\infty} \chi_{i}(\mathbf{R}) \Psi_{i}(\mathbf{R}, \mathbf{r})
$$

Here the functions $\chi_{i}(\mathbf{R})$ serve as $\mathbf{R}$-dependent expansion coefficients for the complete set of electronic wave functions $\Psi_{i}(\mathbf{R}, \mathbf{r})$. This expression is known as the Born representation. After substituting the wave function in the Schrödinger equation 2.1 by the general wave function 2.3 multiplying from the left by a specific $\Psi_{j}^{*}(\mathbf{R}, \mathbf{r})$ from the complete set of electronic wave functions and integrating over the electronic coordinates one arrives at

$$
\left[\hat{T}_{n}+\mathcal{E}_{j}(\mathbf{R})\right] \chi_{i}(\mathbf{R})-\sum_{i} \Lambda_{j i} \chi_{i}(\mathbf{R})=E \chi_{j}(\mathbf{R})
$$

This form of the equation 2.1 is more intuitive, including the eigenenergies $\mathcal{E}_{j}(\mathbf{R})$ of the electronic Hamiltonian. The term $\Lambda_{j i}$ corresponds to the so-called nonadi- 
abatic couplings, which describe the coupling between the nuclear and electronic wave functions:

$$
\Lambda_{j i}=\delta_{j i} \hat{T}_{n}-\left\langle\Psi_{j}(\mathbf{R}, \mathbf{r})\left|\hat{T}_{n}\right| \Psi_{i}(\mathbf{R}, \mathbf{r})\right\rangle
$$

Since the $\mathbf{r}$ dependence is integrated out the $\Lambda_{j i}$ are operators acting only on the $\mathbf{R}$ space.

Practically a complete basis in 2.3 cannot be used and one has to truncate the sum considerably. One can take into account a small number of coupled states and truncate the sum accordingly. This approximation is called the group BornOppenheimer approximation. In the case when only a single product in the sum is taken into account we talk about Born-Oppenheimer approximation. Even though only one term is considered, the coupling element $\Lambda_{11}$ is still maintained in the equation 2.5. Omitting this element we would arrive at the so-called adiabatic approximation, where:

$$
\left[\hat{T}_{n}+\mathcal{E}(\mathbf{R})\right] \chi(\mathbf{R})=E \chi(\mathbf{R}) .
$$

This equation in essence describes the motion of the nuclei moving on the potential energy surface $\mathcal{E}(\mathbf{R})$. This is a good approximation in most of the cases, mainly due to the fact that the electron mass is much smaller than the mass of nuclei and the fast moving electrons follow the comparably slow movement of the nuclei virtually instantaneously. Another condition that has to be fulfilled, in order to keep the coupling elements $\Lambda_{j i}$ small, resp. negligible, is that the electronic energy levels have to be well enough separated in energy.

\subsubsection{Wave Functions of the Nuclei}

The best way to illustrate the dynamics of the nuclei on the potential energy surface (PES) is through the harmonic approximation of the nuclear motion. In the closest vicinity of a minimum, denoted as $\mathbf{R}^{0}$, the PES can be approximated by a multidimensional quadratic form from the Taylor expansion:

$$
\mathcal{E}(\mathbf{R})=\mathcal{E}\left(\mathbf{R}^{0}\right)+\sum_{m, n=1}^{3 N_{n u c l}} \frac{1}{2} k_{m n} \Delta R_{m} \Delta R_{n}
$$


where $\Delta R_{m}=R_{m}^{0}-R_{m}$ is a small deviation from the position of the minimum $R_{m}^{0}$. After a transformation to the mass weighted normal mode coordinates

$$
\Delta R_{n}=\sum_{\alpha} \frac{A_{n \alpha} Q_{\alpha n}}{\sqrt{M_{n}}}
$$

which conveniently exclude the dependence on mass from the final Hamiltonian, with $A_{n \alpha}$ the normal mode transformation matrix element. The approximate Hamiltonian for the nuclear motion around the minimum then becomes:

$$
H_{\text {approx }}=\mathcal{E}(\mathbf{Q}=0)+\frac{1}{2} \sum_{\alpha}\left(P_{\alpha}^{2}+\omega_{\alpha}^{2} Q_{\alpha}^{2}\right)
$$

which corresponds to a system of independent harmonic oscillators vibrating around the equilibrium position $\mathbf{Q}=0$. The eigenenergies of this system are:

$$
\epsilon_{N}=\sum_{\alpha} \hbar \omega_{\alpha}\left(N_{\alpha}+\frac{1}{2}\right)
$$

where $N_{\alpha}$ is the number of vibrational quanta in each mode and $\omega_{\alpha}$ the normal mode frequencies. This approximation helps us not only study the equilibrium vibrational states but also simplify vibrational dynamics on a PES.

Generally after a vertical excitation the molecule does not end up in the vibrational ground state of the excited state PES, but rather moves along this surface described by the Hamiltonian $H_{n u c l}=\hat{T}_{n}+\mathcal{E}(\mathbf{R})$, where the PES $\mathcal{E}(\mathbf{R})$ is $3 N-6$ dimensional. Practically solving the electronic Schödinger equation using quantum chemistry methods for a large number of dimensions becomes computationally prohibitively difficult. Therefore the vibrational degrees of freedom have to be separated into active coordinates $\mathbf{R}_{a}$, which take part in the geometry change during the dynamics, and spectator coordinates $\mathbf{R}_{s}$, which are considered fixed around their equilibrium positions $\mathbf{R}_{s}^{0}\left(\mathbf{R}_{a}\right)$ dependent on the active coordinate $\mathbf{R}_{a}$. The spectator coordinates can then be treated in the harmonic approximation, introducing their displacements $Q_{(s)}$ analogically to 2.8 . The Hamiltonian 
for the nuclear motion on the PES can be then written as:

$$
\begin{aligned}
H_{\text {nucl }}=\sum_{n=1}^{N_{a}} \frac{p_{n}^{2}}{2 M_{n}}+\mathcal{E}\left(\mathbf{R}_{a}, \mathbf{R}_{s}\right)+ \\
\quad \frac{1}{2} \sum_{\alpha=1}^{3 N-N_{a}-6}\left[P_{\alpha}^{2}+\omega_{\alpha}^{(s) 2} Q_{\alpha}^{(s) 2}-2 f_{\alpha} Q_{\alpha}^{(s) 2}\right]
\end{aligned}
$$

where

$$
\mathbf{f}\left(\mathbf{R}_{s}\right)=-\left(\frac{\partial \mathcal{E}\left(\mathbf{R}_{a}, \mathbf{R}_{s}\right)}{\partial \mathbf{R}_{s}}\right)_{\mathbf{R}_{s}=\mathbf{R}_{s}^{0}\left(\mathbf{R}_{a}\right)}
$$

describes the forces on the spectator atoms due to the motion of the active atoms.

\subsubsection{Electron Wave Functions and Electron Density}

Finding the solution for the stationary Schrödinger equation $\hat{H}_{e} \Psi=\mathcal{E} \Psi$ given by the electron part $\hat{H}_{e}$ of the Hamiltonian 2.2 is not a simple task. Since it is practically impossible to solve the Schrödinger equation for a many particle system exactly one has to resort to a number of approximations which will be shortly summarized in this Section.

One of the basic tasks of any quantum chemistry calculation is finding the ground state energy of the system. This is facilitated by the use of the variational principle. When the electronic Hamiltonian is applied to an arbitrary many electron wave function of an appropriate form we get the energy as:

$$
E\left[\Psi_{a r b}\right]=\frac{\left\langle\Psi_{a r b}\left|\hat{H}_{e}\right| \Psi_{a r b}\right\rangle}{\left\langle\Psi_{a r b} \mid \Psi_{a r b}\right\rangle}
$$

Naturally, this functional has a minimum at the ground state energy $\mathcal{E}_{G S}$ when $\Psi_{\text {arb }} \equiv \Psi_{G S}$. Building on this, a method of finding the ground state energy and wave function of the Hamiltonian can be devised. First one takes a trial wave function $\Psi_{a r b}$ of suitable mathematical form containing a number of parameters. In the second step one finds the correct set of parameters which minimize the energy.

The electronic wave function $\Psi$ must satisfy appropriate boundary conditions - it must decay towards zero at infinity for atoms and molecules or satisfy specific periodic boundary conditions for solids. Additionally, it should be normalized to give the correct number of electrons. Another condition originates in the relativis- 
tic quantum field theory and states that an electron wave function must be an antisymmetric function. This means that the many electron wave function must change sign when the coordinates, for both position and spin, of any two electrons are interchanged.

The search for the solution to Hamiltonian $\hat{H}_{e}$ would be greatly simplified if it was possible to approximate it by a sum over effective one electron Hamiltonians $\hat{H}_{e}=\sum_{i} \hat{h}_{i}$, since then the total wave function would have a form of a product of one electron wave functions - orbitals $-\Psi=\prod_{i} \psi_{i}$ which is also called a Hartree product wave function. However, a simple product of one electron orbitals does not obey the antisymmetry condition. Instead, one has to build a Slater determinant of orbital functions:

$$
\Psi_{S D}=\frac{1}{\sqrt{N !}}\left|\begin{array}{cccc}
\psi_{1}(1) & \psi_{2}(1) & \ldots & \psi_{N}(1) \\
\psi_{1}(2) & \psi_{2}(2) & \ldots & \psi_{N}(2) \\
\vdots & \vdots & \ddots & \vdots \\
\psi_{1}(N) & \psi_{2}(N) & \ldots & \psi_{N}(N)
\end{array}\right|
$$

where the factor $\frac{1}{\sqrt{N !}}$ guarantees the normalization condition $\left\langle\Psi_{S D} \mid \Psi_{S D}\right\rangle=1$. The Slater determinant is the electronic wave functions expression of choice in quantum chemistry calculations. In the following, the use of this function in the most basic of ab-initio methods - the Hartree-Fock method — will be summarized.

Let us now take a wave function $\Psi_{H F}$ in the form of a normalized Slater determinant 2.14 with the molecular orbital $\psi_{i}(\mathbf{r})$ being products of orthonormal spatial orbital $\phi_{i}(\mathbf{r})$ and spin function $\sigma_{i}(s)=\alpha(s)$ or $\beta(s)$. For clarity, the terms in the electronic Hamiltonian will be separated according to the number of electron indices $\hat{H}_{e}=\sum_{i} \hat{h}_{i}+\sum_{i} \sum_{j} \hat{g}_{i j}$, where $\hat{h}_{i}$ is the sum of one-electron kinetic energy and electron-nuclear attraction and $\hat{g}_{i j}$ is the electron-electron repulsion. The total energy can then be described by the form:

$$
E_{H F}\left[\Psi_{H F}\right]=\left\langle\Psi_{H F}\left|\hat{H}_{e}\right| \Psi_{H F}\right\rangle=\sum_{i=1}^{N} H_{i}+\frac{1}{2} \sum_{i, j=1}^{N}\left(J_{i j}-K_{i j}\right)
$$

with $H_{i}=\int d \mathbf{r} \psi_{i}^{*}(\mathbf{r}) \hat{h}_{i} \psi_{i}(\mathbf{r})$, the Coulomb integral

$$
J_{i j}=\iint d \mathbf{r} d \mathbf{r}^{\prime} \psi_{i}(\mathbf{r}) \psi_{i}^{*}(\mathbf{r}) \hat{V}_{e e} \psi_{j}^{*}\left(\mathbf{r}^{\prime}\right) \psi_{j}\left(\mathbf{r}^{\prime}\right)
$$


and exchange integral

$$
K_{i j}=\iint d \mathbf{r} d \mathbf{r}^{\prime} \psi_{i}^{*}(\mathbf{r}) \psi_{j}(\mathbf{r}) \hat{V}_{e e} \psi_{i}\left(\mathbf{r}^{\prime}\right) \psi_{j}^{*}\left(\mathbf{r}^{\prime}\right)
$$

which are all real and satisfy $J_{i j} \geq K_{i j} \geq 0$. The Hartree-Fock ground state energy of a system is obtained by minimizing equation 2.15 over all possible orthogonal molecular orbitals.

An important part of quantum chemistry calculations is to choose a suitable form of molecular orbitals $\psi(\mathbf{r})$ that build the Slater determinant. Theoretically, the trial molecular orbitals can be expanded in terms of a complete basis set, thus the true minimum of 2.13 (over the set of single-determinant wave functions) could be find by optimizing the expansion coefficients. However, actual calculations can be performed only using a finite basis set, which introduces an additional approximation in the framework of quantum chemistry calculations. Naturally it follows that the more the basis set is truncated the poorer the results of the energy minimum calculation.

The exact solution of the Schrödinger equation for hydrogen atom serves as a foundation for the introduction of Slater Type Orbitals (STO). They have the form

$$
\chi_{\zeta, n, l, m}(r, \theta, \phi)=N Y_{l, m}(\theta, \phi) r^{n-1} e^{-\zeta r}
$$

where $Y_{l, m}$ are the spherical harmonics and $N$ is a normalization constant. Here the correct character of the wave functions, namely the radial nodes, are created by using linear combinations of STOs. The advantage of this basis set is its correct exponential dependence $e^{-\zeta r}$ which prompts a fast convergence of the energy calculation with the increasing number of basis functions. However, the disadvantage of the Slater functions - nonexistence of analytical expressions for three- and four-center two-electron integrals - deems them useful only in a narrow range of problems, in most of the cases only dealing with atomic and diatomic systems, or else require complicated numerical integration procedures.

Preferred for their analytical properties are the Gaussian Type Orbitals (GTO) of the form

$$
\begin{array}{r}
\chi_{\zeta, n, l, m}(r, \theta, \phi)=N Y_{l, m}(\theta, \phi) r^{2 n-2-l} e^{-\zeta r^{2}} \\
\chi_{\zeta, n, l, m}(x, y, z)=N x^{l_{x}} y^{l_{y}} z^{l_{z}} e^{-\zeta r^{2}}
\end{array}
$$


Although they do not have the correct cusp behavior of the STOs near the nucleus and the fall-off $e^{-\zeta r^{2}}$ with increasing $r$ is too fast, the advantage of analytical twoelectron integrals makes them the basis set of choice for most of quantum chemistry calculations. The drawbacks of GTOs can be compensated for by increasing the number of basis functions used which is computationally preferable to numerical integration. Since the application of GTOs in detailed x-ray scattering simulation is discussed later in this thesis, let us now describe their construction with some detail here.

The basic work on the so-called split valence molecular orbitals was done by Pople (see for example [12], 13]). The molecular orbitals $\psi_{i}$ in the Slater determinant 2.14 are composed of basis functions $\psi_{i}=\sum_{\mu} c_{\mu i} \phi_{i}$. Here the functions $\phi_{i}$ consist of a fixed set of Gaussian functions, while the coefficients $c_{\mu i}$ are optimized to minimize the total energy of the molecule. In the case of the first row elements these so-called contracted basis functions are built as a sum over single, also called primitive, Gaussian functions

$$
\begin{aligned}
\phi_{1 s}(\mathbf{r}) & =\sum_{k=1}^{N_{1}} d_{1 s, k} g_{s}\left(\alpha_{1 k}, \mathbf{r}\right), & & \\
\phi_{2 s}^{\prime}(\mathbf{r}) & =\sum_{k=1}^{N_{2}^{\prime}} d_{2 s, k}^{\prime} g_{s}\left(\alpha_{2 k}^{\prime}, \mathbf{r}\right), & \phi_{2 p x}^{\prime}(\mathbf{r}) & =\sum_{k=1}^{N_{2}^{\prime}} d_{2 p x, k}^{\prime} g_{p x}\left(\alpha_{2 k}^{\prime}, \mathbf{r}\right), \\
\phi_{2 s}^{\prime \prime}(\mathbf{r}) & =\sum_{k=1}^{N_{2}^{\prime \prime}} d_{2 s, k}^{\prime \prime} g_{s}\left(\alpha_{2 k}^{\prime \prime}, \mathbf{r}\right), & \phi_{2 p x}^{\prime \prime}(\mathbf{r}) & =\sum_{k=1}^{N_{2}^{\prime \prime}} d_{2 p x, k}^{\prime \prime} g_{p x}\left(\alpha_{2 k}^{\prime \prime}, \mathbf{r}\right),
\end{aligned}
$$

with the functions $\psi$ representing the core shell, $\phi^{\prime}$ and $\phi^{\prime \prime}$ the inner and outer valence shell, the coefficients $\alpha_{\nu}$ and $d_{\nu, k}$ are fixed to minimize the ground state energy for the given atom, and $g_{s}, g_{p x}$ are Gaussian functions

$$
g_{s}=\frac{2 \alpha^{3 / 4}}{\pi} \exp \left(-\alpha \mathbf{r}^{2}\right), \quad g_{p x}={\frac{128 \alpha^{5}}{\pi^{3}}}^{1 / 4} \exp \left(-\alpha \mathbf{r}^{2}\right) .
$$

After choosing the number of primitive basis functions $N_{1}, N_{2}^{\prime}$ and $N_{2}^{\prime \prime}$ one arrives at the corresponding split-valance basis set representation $N_{1}-N_{2}^{\prime} N_{2}^{\prime \prime}$ G, the widely used basis sets being $4-31 \mathrm{G}$ or $6-31 \mathrm{G}$. In another words, in the case of first row elements, each molecular orbital in this representation is described by a core $1 s$, inner valence $2 s$ and $2 p x$ orbitals and outer valence $2 s$ and $2 p x$ orbitals, with the 
weights $c_{\mu i}$ determining the prevalent character of this orbital.

\section{$2.2 \quad$ X-Ray Scattering}

This Section summarizes the main principles governing x-ray scattering when studied quantum mechanically. Details on the theory of time-resolved diffraction as well as examples of time-resolved measurements performed using synchrotron sources can be found for example in the review work [14] by Helliwell, or References [15, 16, 17. Additionally, x-ray scattering from molecular systems with random orientations of molecules - liquids or gases and systems of aligned molecules — will be described in detail.

\subsubsection{Time-Resolved X-Ray Scattering}

For start, let us assume that the system under consideration is a single molecule in the x-ray field. This assumption will be later dropped in order to study the scattering from large assemblies of molecules.

Quantum mechanically x-ray scattering is expressed in terms of the following Hamiltonian:

$$
\hat{H}=\hat{H}_{r}+\sum_{j} \frac{\mathbf{p}_{j}}{2 m_{j}}+V-\sum_{j} \frac{e_{j}}{m_{j} c} \mathbf{A}_{j} \cdot \mathbf{p}_{j}+\sum_{j} \frac{e_{j}^{2}}{2 m_{j} c^{2}}\left|\mathbf{A}_{j}\right|^{2}
$$

with $\hat{H}_{r}$ corresponding to the Hamiltonian of the radiation field, $\mathbf{p}_{j}$ the momentum operator of the $j$-th particle and the vector potential $\mathbf{A}_{j}$ :

$$
\mathbf{A}_{j}=\sum_{k} \hat{e}_{k}\left(\frac{2 \pi \hbar c^{2}}{\omega_{k} L^{3}}\right)^{1 / 2}\left[\hat{a}_{k} \exp \left(i \mathbf{k}_{k} \cdot \mathbf{r}_{j}\right)+\hat{a}_{k}^{*} \exp \left(-i \mathbf{k}_{k} \cdot \mathbf{r}_{j}\right)\right]
$$

with $\hat{e}_{k}$ and $\mathbf{k}_{k}$ being the polarization and propagation vectors of the mode $k$ and frequency $\omega_{k}$, and the annihilation and creation operators $\hat{a}_{k}, \hat{a}_{k}^{*}$.

From the time-dependent perturbation theory it follows that the transition rate for this kind of processes can be expressed as:

$$
\begin{gathered}
W_{F I}=\frac{2 \pi}{\hbar}\left|\left\langle F\left|H^{(2)}\right| I\right\rangle\right|^{2} \rho(E)= \\
\frac{2 \pi}{\hbar}\left(\frac{2 \pi \hbar e^{2}}{m \omega_{k} L^{3}}\right)^{2}\left(n_{k}+1\right) n_{k_{0}}\left|\left\langle f\left|\sum_{j} \exp \left(i \mathbf{q} \cdot \mathbf{r}_{j}\right)\right| i\right\rangle\left(\hat{e}_{k} \cdot \hat{e}_{k 0}\right)\right|^{2}
\end{gathered}
$$


where $H^{(2)}$ corresponds to the last term of the Hamiltonian 2.24 .

$|I\rangle=\left|\epsilon_{i}, n_{k_{0}} \hbar \omega_{k_{0}}, n_{k} \hbar \omega_{k}\right\rangle$ is the initial state of the system,

$|F\rangle=\left|\epsilon_{f},\left(n_{k_{0}}-1\right) \hbar \omega_{k_{0}},\left(n_{k}+1\right) \hbar \omega_{k}\right\rangle$ is the final state of the system and $\rho(E)$ is the number of radiation oscillators per unit energy. The differential scattering cross section will be:

$$
\frac{d \sigma}{d \Omega}=\left(\frac{e^{2}}{m c^{2}}\right)^{2}\left(\hat{e}_{k} \cdot \hat{e}_{k 0}\right)^{2}\left|\left\langle f\left|\sum_{j} \exp \left(i \mathbf{q} \cdot \mathbf{r}_{j}\right)\right| i\right\rangle\right|^{2}
$$

here $|f\rangle$ and $|i\rangle$ correspond to the wave functions of the final and initial state. In the case of x-ray scattering and diffraction the initial state $|i\rangle$ of the molecule is equal to the final state, the radiation potential does not cause any excitations. For a molecule these wave function would be of the form of the total wave function including electronic and nuclear wave functions:

$$
\Phi(\mathbf{R}, \mathbf{r})=\Psi_{e l}(\mathbf{r} \mid \mathbf{R}) \chi_{n u c l}(\mathbf{R})
$$

with $\Psi_{e l}$ being implicitly $R$ dependent and $\chi_{n u c l}$ build from vibrational wave functions which in the harmonic approximation (see the Section 2.1.2 have the form $\chi_{n u c l}=\prod_{j=1}^{M} \xi_{j}\left(Q_{j}\right), M$ denoting the degrees of freedom in vibrational coordinates. These vibrational coordinates arise from the harmonic approximation in which vibrational coordinates are separated. The use of this approach helps to clarify the influence of the molecular movement on the differential cross section and scattering intensity.

The main term in the $d \sigma / d \Omega$ expression can be expanded as:

$$
\begin{aligned}
& \langle f|\exp (i \mathbf{q} \cdot \hat{\mathbf{r}})| i\rangle= \\
& \int d Q_{1} \ldots d Q_{M} d \mathbf{r} \xi_{1}^{*}\left(Q_{1}\right) \ldots \xi_{M}^{*}\left(Q_{M}\right) \Psi_{e l}^{*}(\mathbf{r} \mid \mathbf{Q})(\exp (i \mathbf{q} \cdot \hat{\mathbf{r}})) \xi_{1}\left(Q_{1}\right) \ldots \xi_{M}\left(Q_{M}\right) \Psi_{e l}(\mathbf{r} \mid \mathbf{Q})= \\
& \int d Q_{1} \ldots d Q_{M} \mid \xi_{1}\left(\left.Q_{1}\right|^{2} \ldots\left|\xi_{M}\left(Q_{M}\right)\right|^{2} \int d \mathbf{r} \rho(\mathbf{r} \mid \mathbf{Q}) \exp (i \mathbf{q} \cdot \mathbf{r})\right.
\end{aligned}
$$

where $\hat{\mathbf{r}}$ corresponds to the electron position operator.

In many practical cases some modes in the vibrational space are strongly confined. By substituting the wave functions of these modes for the delta function $\xi_{j}\left(Q_{j}\right)=\delta\left(Q_{j 0}\right)$ these modes would be easily integrated out of the expression by 
substituting the corresponding dependence on the vector $\mathbf{Q}$ with the positions $Q_{j 0}$ in the electron density term $\left.\rho(\mathbf{r} \mid \mathbf{Q})\right|_{Q_{j}=Q_{j 0}}$.

In order to be able to deal with the equation 2.29 one has to consider the implicit dependence of the electron density on the nuclear degree of freedom. From quantum chemistry calculations one obtains a set of $\rho(\mathbf{r})$ each corresponding to a different $\mathbf{Q}$ rather than explicit function $\rho(\mathbf{r}, \mathbf{Q})$. From this follows that we have to deal with the vibrational degree of freedom through numerical integration. A sufficiently small number of vibrational modes $m$ which are considered having an effect on the scattering intensity can be assessed by numerical integration and the differential cross section can be expressed in the form:

$$
\begin{aligned}
& \left.\left.\frac{d \sigma}{d \Omega} \propto\left|\int d Q_{1} \ldots d Q_{m}\right| \xi_{1}\left(Q_{1}\right)\right|^{2} \ldots\left|\xi_{m}\left(Q_{m}\right)\right|^{2} \mathcal{F} \mathcal{T}[\rho(\mathbf{r} \mid \mathbf{Q})]\right|^{2}= \\
& \left|\sum_{a} \Delta Q_{1} \ldots \sum_{c} \Delta Q_{m} p_{1}\left(a \Delta Q_{1}\right) \ldots p_{m}\left(c \Delta Q_{m}\right) \mathcal{F} \mathcal{T}\left[\rho\left(\mathbf{r} \mid a \Delta Q_{1}, \ldots, c \Delta Q_{m}\right)\right]\right|^{2}
\end{aligned}
$$

where $\mathcal{F} \mathcal{T}[\rho(\mathbf{r} \mid \mathbf{Q})]$ denotes the Fourier transformation of the electron density in a given point $\mathbf{Q}$ in the space of vibrational coordinates and indexes $a, \ldots, c$ run through the discretized probability distribution functions of the vibrational wave functions $p_{j}\left(Q_{j}\right)=\left|\xi_{j}\left(Q_{j}\right)\right|^{2}$. Practically the number of contributing modes $m$ stays small since it is mainly the weakly confined ones that significantly change the overall intensity.

Having the implicit $\mathbf{Q}$ dependence of the electron density in mind we can now move on to studying its influence on the time-dependence of $\mathrm{x}$-ray scattering. This enters the equations through the nuclear degree of freedom as $\xi(\mathbf{Q}, t)$ and $p_{j}\left(Q_{j}, t\right)=\left|\xi_{j}\left(Q_{j}, t\right)\right|^{2}$ and so also the electron density $\rho(\mathbf{r} \mid \mathbf{Q}, t)$.

\subsubsection{Electron Density and its Fourier Transformation}

The usual way of dealing with the electron density part of the differential-cross section 2.30 is an approximation by introducing the atomic scattering factors $f$. These practically approximate the electron density by a suitably normalized delta function in the positions of the nuclei. In this way, any effect on the scattering intensity arising from the realistic electron density distribution, as are for example 
bonds or electron delocalization in aromatic molecules, is neglected. However, by using the GTO basis set expression for the molecular orbitals and electron density, as introduced in the Section 2.1.3, one can include the details of electron density distribution in the differential-cross section expression. In the Hartree-Fock theory and Kohn-Sham density functional theory the electron density can be expressed in terms of the molecular orbitals as:

$$
\rho(\mathbf{r})=\sum_{i=1}^{n_{\mathrm{occ}}} b_{i}\left|\psi_{i}(\mathbf{r})\right|^{2}
$$

with occupation numbers $b_{i}=1,2$ and the number of occupied orbitals $n_{\text {occ }}$. The orbitals are expanded as:

$$
\psi_{i}(\mathbf{r})=\sum_{\nu=1}^{N} C_{\nu i} \phi_{\nu}(\mathbf{r})
$$

where the $N$ real basis functions $\phi_{\nu}$ centered at $\mathbf{r}_{\nu}=\left(x_{\nu}, y_{\nu}, z_{\nu}\right)$ are defined as

$$
\phi_{\nu}(\mathbf{r})=\mathcal{N}\left(x-x_{\nu}\right)^{l_{\nu}}\left(y-y_{\nu}\right)^{m_{\nu}}\left(z-z_{\nu}\right)^{n_{\nu}} \exp \left(-\alpha_{\nu}\left(\mathbf{r}-\mathbf{r}_{\nu}\right)^{2}\right)
$$

with normalization constant $\mathcal{N}$. Here, $(l+m+n)=0,1,2, \ldots$ denotes $s-, p$-, $d$-type functions etc. Since the product of two Gaussians is a Gaussian, we obtain for the electron density

$$
\begin{aligned}
& \rho(\mathbf{r})=\sum_{i=1}^{n_{o c c}} \sum_{\mu=1}^{N} \sum_{\nu=1}^{N} b_{i} C_{\mu i} C_{\nu i} \phi_{\mu}(\mathbf{r}) \phi_{\nu}(\mathbf{r}) \\
& =\sum_{i=1}^{n_{\text {occ }}} \sum_{\mu=1}^{N} \sum_{\nu=1}^{N} b_{i} C_{\mu i} C_{\nu i} \exp \left(\frac{\alpha_{\mu} \alpha_{\nu}}{\alpha_{\mu}+\alpha_{\nu}}\left(\mathbf{r}_{\mu}-\mathbf{r}_{\nu}\right)^{2}\right)\left(x-x_{\mu}\right)^{l_{\mu}}\left(x-x_{\nu}\right)^{l_{\nu}} \\
& \left(y-y_{\mu}\right)^{m_{\mu}}\left(y-y_{\nu}\right)^{m_{\nu}}\left(z-z_{\mu}\right)^{n_{\mu}}\left(z-z_{\nu}\right)^{n_{\nu}} \exp \left(-\alpha_{\mu \nu}\left(\mathbf{r}-\mathbf{r}_{\mu \nu}\right)^{2}\right) \\
& =\sum_{i=1}^{n_{o c c}} \sum_{\mu=1}^{N} \sum_{\nu=1}^{N} b_{i} C_{\mu i} C_{\nu i} \prod_{r^{\prime}=x, y, z}\left(r^{\prime}-r_{\mu}^{\prime}\right)^{p_{r^{\prime}, \mu}}\left(r^{\prime}-r_{\nu}^{\prime}\right)^{p_{r^{\prime}, \nu}} \exp \left(-\alpha_{\mu \nu}\left(r^{\prime}-r_{\mu \nu}^{\prime}\right)^{2}\right)
\end{aligned}
$$

with

$$
\alpha_{\mu \nu}=\alpha_{\mu}+\alpha_{\nu}
$$

and

$$
\mathbf{r}_{\mu \nu}=\frac{\alpha_{\mu} \mathbf{r}_{\mu}+\alpha_{\nu} \mathbf{r}_{\nu}}{\alpha_{\mu}+\alpha_{\nu}}
$$


The Fourier transformation (FT) of the electron density is then given by

$$
\begin{aligned}
\mathcal{F}_{\mathbf{k}}[\rho(\mathbf{r})]= & \sum_{i=1}^{n_{\text {occ }}} \sum_{\mu=1}^{N} \sum_{\nu=1}^{N} b_{i} C_{\mu i} C_{\nu i} \\
& \prod_{r^{\prime}=x, y, z} \mathcal{F}_{k_{r^{\prime}}}\left\{\left(r^{\prime}-r_{\mu}^{\prime}(t)\right)^{p_{r^{\prime}, \mu}}\left(r^{\prime}-r_{\nu}^{\prime}(t)\right)^{p_{r^{\prime}, \nu}} \exp \left(-\alpha_{\mu \nu}\left(r^{\prime}-r_{\mu \nu}^{\prime}\right)^{2}\right)\right\} \\
= & \sum_{i=1}^{n_{o c c}} \sum_{\mu=1}^{N} \sum_{\nu=1}^{N} b_{i} C_{\mu i} C_{\nu i} \\
& \prod_{r^{\prime}=x, y, z} \mathcal{F}_{k_{r^{\prime}}}\left\{\sum_{j=0}^{p_{r^{\prime}, \mu}+p_{r^{\prime}, \nu}} c_{j} r^{\prime p_{r^{\prime}, \mu}+p_{r^{\prime}, \nu}-j} \exp \left(-\alpha_{\mu \nu}\left(r^{\prime}-r_{\mu \nu}^{\prime}(t)\right)^{2}\right)\right\}
\end{aligned}
$$

with $c_{j}=f\left(r_{\mu}^{\prime}, r_{\nu}^{\prime}\right)$ and $c_{0}=1$.

The FT of a Gaussian function is again a Gaussian function:

$$
\mathcal{F} \mathcal{T}_{\mathbf{k}}\left[\exp \left(-\alpha\left(\mathbf{r}-\mathbf{r}_{0}\right)^{2}\right)\right]=\sqrt{\frac{\pi}{\alpha}} \exp \left(-\frac{\mathbf{k}^{2}}{4 \alpha}\right) \exp \left(i \mathbf{k r}_{0}\right)
$$

From the differentiation theorem of the FT,

$$
\mathcal{F T}_{k}[d f(r) / d r]=i k \mathcal{F}_{k}[f(r)]
$$

we can easily calculate the FT of higher angular momentum atomic orbitals. For the FTs of the functions $f_{l}\left(r^{\prime}\right)=r^{\prime l} \exp \left(-\alpha r^{\prime 2}\right)$ we obtain

$$
\mathcal{F}_{k}\left[f_{l+1}\left(r^{\prime}\right)\right]=\frac{i k}{2 \alpha} \mathcal{F}_{k}\left\{f_{l}\left(r^{\prime}\right)\right\}-\frac{l}{2 \alpha} \mathcal{F} \mathcal{T}_{k}\left[f_{l-1}\left(r^{\prime}\right)\right]
$$

In particular, we find for the terms in Eq. 2.37

$$
\begin{gathered}
\mathcal{F} \mathcal{T}_{k}\left[r^{\prime} e^{-\alpha\left(r^{\prime}-r_{0}^{\prime}\right)^{2}}\right\}=\left(\frac{i k}{2 \alpha}+r_{0}^{\prime}\right) \mathcal{F}_{k}\left\{e^{-\alpha\left(r^{\prime}-r_{0}^{\prime}\right)^{2}}\right] \\
\mathcal{F}_{k}\left[r^{\prime 2} e^{-\alpha\left(r^{\prime}-r_{0}^{\prime}\right)^{2}}\right]=\left(\left(\frac{i k}{2 \alpha}+r_{0}^{\prime}\right)^{2}+\frac{1}{2 \alpha}\right) \mathcal{F} \mathcal{T}_{k}\left[e^{-\alpha\left(r^{\prime}-r_{0}^{\prime}\right)^{2}}\right],
\end{gathered}
$$

and

$$
\mathcal{F}_{k}\left[r^{\prime 3} e^{-\alpha\left(r^{\prime}-r_{0}^{\prime}\right)^{2}}\right]=\left(\frac{i k}{2 \alpha}+r_{0}^{\prime}\right)\left[\left(\frac{i k}{2 \alpha}+r_{0}^{\prime}\right)^{2}+\frac{3}{2 \alpha}+3\right] \mathcal{F}_{k}\left\{e^{-\alpha\left(r^{\prime}-r_{0}^{\prime}\right)^{2}}\right\}
$$

for $p$-, $d$ - and $f$-type Gaussian functions. These results can also be obtained from 
the equation

$$
\mathcal{F T}_{k}\left[r^{\prime n} f\left(r^{\prime}\right)\right]=(-i)^{n} \frac{\mathrm{d}^{(n)} \mathcal{F}_{k}\left\{f\left(r^{\prime}\right)\right\}}{\mathrm{d} k^{n}} .
$$

Generally, the FT of the polynomial expression of the density 2.34 has the form:

$$
\mathcal{F} \mathcal{T}_{\mathbf{k}}[\rho(\mathbf{r})]=\sum_{j} C_{j} k_{x}^{l_{j}} k_{y}^{m_{j}} k_{z}^{n_{j}} \exp \left(-D_{j} \mathbf{k}^{2}-i \mathbf{k r}_{\mu \nu}\right) .
$$

If we are interested in the x-ray scattering from a liquid sample, the power of the absolute value of the FT of the electron density has to be averaged over all orientations (or spherical angles):

$$
I(k)=\frac{1}{4 \pi} \int_{0}^{2 \pi} d \varphi_{k} \int_{0}^{\pi} d \vartheta_{k} \sin \vartheta_{k}\left|\mathcal{F}_{\mathbf{k}}\{\rho(\mathbf{r})\}\right|^{2}
$$

where the index $k$ in $\varphi_{k}$ and $\vartheta_{k}$ denotes the spherical coordinates in $k$-space. This definite integral contains exponentials of trigonometric functions caused by the shifts $\mathbf{r}_{\mu \nu}$ from Eq. 2.45 and we integrate can it numerically using quadrature in polar coordinates, on a fine enough grid to give converged results.

\subsubsection{Ensemble of Molecules, Gases and Alignment}

Scattering from a random distribution of molecular orientations in gaseous or liquid samples can be assessed straightforwardly by summing the intensity contribution from all molecules in the sample which practically means integrating the expression 2.30 over all angles in spherical coordinates, for all possible orientations of the molecules in the sample:

$$
\begin{aligned}
& \frac{d \sigma}{d \Omega} \propto \frac{1}{4 \pi q^{2}} \int d \psi d \theta \sin \theta\left|\sum_{a} \Delta Q_{1} \ldots \sum_{c} \Delta Q_{m} p_{1}\left(a \Delta Q_{1}\right) \ldots p_{m}\left(c \Delta Q_{m}\right) \mathcal{F} \mathcal{T}(\mathbf{q})[\rho]\right|^{2} \\
& =\frac{1}{2 \pi q^{2}} \int d \theta \sin \theta|f(\mathbf{q})|^{2}
\end{aligned}
$$

Due to this integration information about the structure is lost, the intensity is no longer dependent on the directions of the scattering vector $\mathbf{q}$ but only on its length $q$. In order to boost the structure characteristic part of the scattering intensity distribution, which is the important part of the signal used to identify the molecular structure of the system under study, one has to introduce periodicity 
into the system, as for example is the case in the crystalline systems. In the case of gaseous samples this can be done trough one of the methods of molecular orientation, for example by orienting the molecules in magnetic field or by orienting them in laser field. By doing so we generally arrive at an ensemble of molecules with a preferred orientation of their molecular axis with respect to an the external coordinate system, which corresponds to the external alignment field. The efficiency of the achieved alignment is characterized by a probability distribution of the uncertainty of the angle between the molecular axis and the external alignment field axis.

In order to study a system of aligned identical molecules we have to consider the properties of Fourier transformation with respect to an arbitrary rotation of the function $f(\mathbf{r})$ that is transformed. Any rotation of an object in 3D space can be constructed from three sequential rotations with respect to the coordinate system axis $x, y$ and $z$, which is mathematically expressed by sequential application of the rotation matrices $\hat{R}_{x}(\alpha), \hat{R}_{y}(\beta), \hat{R}_{z}(\gamma)$ corresponding to the rotation angles $\alpha, \beta$ and $\gamma$. The Fourier transformation of a rotated function is then:

$$
\mathcal{F} \mathcal{T}(\mathbf{k})\left[f\left(\hat{R}_{z}(\gamma) \hat{R}_{y}(\beta) \hat{R}_{x}(\alpha) \mathbf{r}\right)\right]=\int d^{3} \mathbf{r} f\left(\hat{R}_{z}(\gamma) \hat{R}_{y}(\beta) \hat{R}_{x}(\alpha) \mathbf{r}\right) e^{-i \mathbf{k r}}
$$

Due to the consecutive application of the rotation matrices to the function $f(\mathbf{r})$ we can constrain the evaluation to one rotation $\hat{R}(\alpha)$ and simplify the integral to $\int d^{3} \mathbf{r} f(\hat{R}(\alpha) \mathbf{r}) e^{-i \mathbf{k r}}$. We apply the substitution $\mathbf{u}=\hat{R}(\alpha) \mathbf{r}$ which can be also written as $u_{i}=\sum_{j} R_{i j} r_{j}$, where for the Jacobian we have $J(\hat{R} \mathbf{r})=\hat{R}$ since rotation is a linear transformation. This leads to $\operatorname{det}(J(\hat{R} \mathbf{r}))=\operatorname{det}(\hat{R})=1$. We also consider that the back substitution is $\mathbf{r}=\hat{R}(-\alpha) \mathbf{u}$ and the property of the rotation matrix $\hat{R}^{T}(\alpha)=\hat{R}(-\alpha)$ from which follows that $\mathbf{k} \cdot(\hat{R}(\alpha) \mathbf{r})=(\hat{R}(-\alpha) \mathbf{k}) \cdot \mathbf{r}$. The Fourier transformation of the rotated function is then:

$$
\int d^{3} \mathbf{r} f(\hat{R}(\alpha) \mathbf{r}) e^{-i \mathbf{k} \cdot \mathbf{r}}=\int d^{3} \mathbf{u} f(\mathbf{u}) e^{-i \mathbf{k} \cdot(\hat{R}(-\alpha) \mathbf{u})}=\int d^{3} \mathbf{u} f(\mathbf{u}) e^{-i(\hat{R}(\alpha) \mathbf{k}) \cdot \mathbf{u}}
$$

from which we can conclude that for a general expression for the Fourier transformation of rotated function:

$$
\mathcal{F} \mathcal{T}(\mathbf{k})\left[f\left(\hat{R}_{z}(\gamma) \hat{R}_{y}(\beta) \hat{R}_{x}(\alpha) \mathbf{r}\right)\right]=\mathcal{F} \mathcal{T}\left(\hat{R}_{z}(\gamma) \hat{R}_{y}(\beta) \hat{R}_{x}(\alpha) \mathbf{k}\right)[f(\mathbf{r})]
$$


Let us now consider a system of $N$ identical molecules aligned with respect to their molecular axis with a probability distribution $p(\alpha)$ of the uncertainty in alignment angle $\alpha$. In order to maintain clarity and simplicity of the expressions we will in the following omit the molecular wave function contribution to the total scattering. An ensemble of aligned identical molecules with the electron density $\rho(\mathbf{r})$ can be simply mathematically expressed as $\sum_{i=1}^{N} \rho\left(\hat{R}\left(\alpha_{i}\right) \mathbf{r}\right)$ and the total differential cross-section and a sum over the contributions from all molecules which leads to:

$$
\begin{aligned}
& \frac{d \sigma}{d \Omega} \propto \sum_{i=1}^{N} c\left(\alpha_{i}\right)\left|\mathcal{F} \mathcal{T}(\mathbf{q})\left[\rho\left(\hat{R}\left(\alpha_{i}\right) \mathbf{r}\right)\right]\right|^{2}=\sum_{i=1}^{N} c\left(\alpha_{i}\right)\left|\mathcal{F} \mathcal{T}\left(\hat{R}\left(\alpha_{i}\right) \mathbf{q}\right)[\rho(\mathbf{r})]\right|^{2}= \\
& \int d \alpha p(\alpha)|\mathcal{F} \mathcal{T}(\hat{R}(\alpha) \mathbf{q})[\rho(\mathbf{r})]|^{2}
\end{aligned}
$$

where $c\left(\alpha_{i}\right)>0$ are the probability distribution coefficients for which it holds $\sum_{i=1}^{N} c\left(\alpha_{i}\right)=1$ and $p(\alpha)$ is the corresponding probability distribution function in the limit $N \rightarrow \infty$ holding $\int_{\alpha} d \alpha p(\alpha)=1$.

Using the expressions presented in this Section the x-ray scattering intensities can be studied in space and time, without neglecting either realistic electron density distribution or vibrational degrees of freedom.

\subsection{Density Functional Theory}

One of the most widely used methods of quantum chemistry that includes electron correlation is the density functional theory (DFT) and the time-dependent density functional theory (TDDFT). These methods conveniently combine computational efficiency with precision. A detailed description of these methods can be found in a number of quantum chemistry texts, for example the works [18] and 9] can be refered to for DFT and works [19, 20] for TDDFT. The following Sections summarize some of the basic principles of these methods and discuss their performance and use when applied to the problems of photo-induced processes and systems in high intensity laser fields. 


\subsubsection{Density Functional Theory}

The basis of the DFT - the Hohenberg-Kohn theorem — states that the ground state properties, most prominently the ground state energy, are completely determined by the ground state electron density trough a one-to-one mapping between the ground state electron density and the external potential 18 .

We have an electronic many particle system described by a Hamiltonian:

$$
\hat{H}=\sum_{i=1}^{N}-\frac{1}{2} \nabla_{i}^{2}+\sum_{i=1}^{N} v\left(\mathbf{r}_{i}\right)+\sum_{i<j}^{N} \frac{1}{\left|\mathbf{r}_{i}-\mathbf{r}_{j}\right|}
$$

which is a conveniently rewritten form of the Hamiltonian 2.2, where

$$
v\left(\mathbf{r}_{i}\right)=-\sum_{\alpha} \frac{Z_{\alpha}}{\left|\mathbf{r}_{i}-\mathbf{R}_{\alpha}\right|}
$$

is the electronic potential of nuclei $Z_{\alpha}$ acting on the electron $i$.

The Hohenberg-Kohn theorem proves that the ground state of an electronic system is completely described by the external potential $v(\mathbf{r})$ and number of electrons $N$. Due to the fact that the electron density $\rho(\mathbf{r})$ determines not only the external potential, up to an additive constant, but also the number of electrons it follows that the knowledge of $\rho(\mathbf{r})$ is sufficient for a complete description of the system, particularly the ground state energy.

Generally, the electron density function $\rho(\mathbf{r})$ must have specific properties. Theoretically, the ground state density is connected to the antisymmetric ground state solution of the electronic Hamiltonian with some external potential $v(\mathbf{r})$. A general density function is called $v$-representable if it is linked to the external potential in this manner. For $v$-representable densities follows from the HohenbergKohn theorem

$$
E_{v}[\rho] \equiv F_{H K}[\rho]+\int v(\mathbf{r}) \rho(\mathbf{r}) d \mathbf{r} \geq E_{v}\left[\rho_{0}\right]
$$

where $F_{H K}[\rho]=\left\langle\Psi\left|\hat{T}+\hat{V}_{e e}\right| \Psi\right\rangle$ and $\rho_{0}$ is the ground state electron density. However, not all densities can be linked to the external potential through a ground state wave function. Rather they can be associated with some antisymmetric wave function of the correct number of particles. This kind of densities are called $N$ representable. Fortunately, it can be shown that the DFT can be formulated to apply to electron densities that satisfy the more general $N$-representability condi- 
tion.

The energy functional is straightforwardly divided into the following parts, which are the kinetic energy functional $T[\rho]$, the $E_{e e}[\rho]$ electron-electron interaction energy part, and the $v[\rho]$ electron-nuclei interaction part, where $E_{e e}[\rho]$ consists of the Coulomb part $J[\rho]$ and the Exchange part $K[\rho]$ terms which implicitly include the correlation energy. Here the Coulomb and $v[\rho]$ parts are given by their classical expressions, while the kinetic energy, exchange and correlation parts are expressed using various models of electron interaction which have undergone a gradual fine-tuning to describe different quantum chemical situations.

In the simplest Thomas-Fermi model ${ }^{21}$, based on the electron density of a noninteracting homogeneous electron gas, the functionals are dependent strictly only on the electron density. The energy functional in this model has a form

$$
\begin{aligned}
& E_{T F}[\rho]=T_{T F}[\rho]+E_{n e}[\rho]+J[\rho]=\frac{3}{10}\left(3 \pi^{2}\right)^{2 / 3} \int d \mathbf{r} \rho^{5 / 3}(\mathbf{r})+ \\
& \sum_{a} \int d \mathbf{r} \frac{Z_{a} \rho(\mathbf{r})}{\left|\mathbf{R}_{a}-\mathbf{r}\right|}+\frac{1}{2} \iint d \mathbf{r} d \mathbf{r}^{\prime} \frac{\rho(\mathbf{r}) \rho\left(\mathbf{r}^{\prime}\right)}{\left|\mathbf{r}-\mathbf{r}^{\prime}\right|}
\end{aligned}
$$

Here $T_{T F}[\rho]$ is the kinetic energy functional, $E_{n e}[\rho]$ the nuclei-electron interaction energy part, and the Coulomb functional $J[\rho]$ is one part of the electron-electron interaction energy.

In this basic Thomas-Fermi model the functionals are dependent strictly only on the local electron density. It provides total energies with the error of $15-50 \%$ and more importantly it does not allow for chemical bonds because of the assumption of the non-interacting homogeneous electron gas. ${ }^{22}$ An improvement of this model considers not only electron density itself but also the gradients of electron density. This is similar to taking Taylor expansion of non-uniform electron densities. It improves the problem with the chemical bonds but the error of this method by far does not approach the accuracy of the wave mechanics methods. The most difficult part of the total energy to approximate with an electron density function is the kinetic energy.

The solution to the problem with kinetic energy functional is sought in the Kohn-Sham formalism. Here the kinetic energy functional is split into two terms from which one is calculated exactly and one represents a small correction. Similarly to the Hartree-Fock orbital method the electron density is expressed in a set 
of orbitals of non-interacting particles:

$$
\rho(\mathbf{r})=\sum_{i=1}^{N}\left|\phi_{i}(\mathbf{r})\right|^{2}
$$

which leads to the expression for the kinetic energy of the corresponding Slater determinant

$$
T_{S}=\sum_{i=1}^{N}\left\langle\phi_{i}\left|-\frac{1}{2} \nabla^{2}\right| \phi_{i}\right\rangle .
$$

These orbitals are chosen to minimize the kinetic energy $T_{S}=\min _{\phi \rightarrow \rho}\left\langle\phi_{i}|\hat{T}| \phi_{i}\right\rangle$ while giving the density $\rho$. In order to understand the level of accuracy of this approximation we can compare it to the exact kinetic energy as expressed in terms of natural orbitals:

$$
\begin{aligned}
& T\left[\rho_{\text {exact }}\right]=\sum_{i=1}^{\infty} n_{i}\left\langle\phi_{i}^{\text {norb }}\left|-\frac{1}{2} \nabla^{2}\right| \phi_{i}^{\text {norb }}\right\rangle \\
& \rho_{\text {exact }}=\sum_{i=1}^{\infty} n_{i}\left\langle\phi_{i}^{\text {norb }} \mid \phi_{i}^{\text {norb }}\right\rangle \\
& N=\sum_{i=1}^{\infty} n_{i}
\end{aligned}
$$

with the orbital occupancy numbers $n_{i} \in[0,1]$. Therefore when the approximate density is expressed as a set of one-electron orbitals

$$
\rho(\mathbf{r})=\sum_{i=1}^{\infty} n_{i}\left\langle\phi_{i} \mid \phi_{i}\right\rangle,
$$

it would correspond to the $T\left[\rho_{\text {exact }}\right]$ if the occupancies were exactly 0 and 1 . The small correction coming from the difference between the kinetic energy of interacting and non-interacting electrons is is usually accounted for in the exchangecorrelation term $E_{x c}[\rho]$ and thus the energy can be generally written as:

$$
E_{D F T}[\rho]=T_{S}[\rho]+v[\rho]+J[\rho]+E_{x c}[\rho]
$$

The DFT exchange and correlation energy, with exchange constituting the largest part of $E_{x c}$, is different from the exchange and correlation energy as defined in the wave function theory. In the wave function theory, the exchange energy is 
defined as the total electron repulsion minus the Coulomb energy, the correlation energy is the difference between the exact energy and the Hartree-Fock energy. They both have short-range and long-range parts with the long-range part of the exchange energy canceling the long range part of the correlation energy. In DFT the exchange and correlation has only short-range part since it depends only on the electron density and the local gradient, therefore by applying the wave mechanics expression for exchange here one would exclude the cancellation of the long-range energy parts.

In order to provide necessary accuracy in the kinetic energy calculation the Kohn-Sham orbitals have to be introduced and so the computational costs of DFT are similar to those of Hartree-Fock calculations. However, the DFT provides additional accuracy due to the inclusion of the electron correlation.

Theoretically, there exists a general $E_{x c}$ functional valid for all system. Practically its exact form is not known and it has to be approximated by different functional forms for different classes of electron systems, e.g. molecular systems, solid state materials etc. There are three main approximation methods for arriving at an expression for this functional.

In the most basic approximation it is assumed that the electron density locally behaves as the electron density of homogeneous electron gas, that is, electron density varies only slowly as a function of the coordinate $\mathbf{r}$. This approximation is called the Local Density Approximation (LDA) or more generally Local Spin Density Approximation (LSDA) in the case when the $\alpha$ and $\beta$ spin densities are not equal. The accuracy of the results provided by this approximation is on average similar the Hartree-Fock method. In the case of molecules the HartreeFock method provides better results, while the LDA is a better approximation for metals.

In order to improve the accuracy one has to account for non-homogeneous electron gas. This is done by making the $E_{x c}$ depend not only on the local electron density but also on the derivatives of the electron density and generally methods using this approach are called Generalized Gradient Approximation (GGA). A number of different GGA functionals was proposed, most notably by Lee, Yang and Parr 23 - the LYP functional, providing the correction for the correlation energy, Becke $^{\sqrt{24}}$ in B or B88 functionals and Perdew and Wang ${ }^{25}$ in PW86 functional providing the corrections for the exchange energy, etc. 
The third class of methods are so called hybrid methods. In this case, the exchange functionals is built from the exact exchange as defined in the HartreeFock method combined with LSDA and GGA exchange, while the correlation is built as a combination of LSDA correlation with an additional GGA term.

\subsubsection{Time-Dependent Density Functional Theory}

The Runge and Gross ${ }^{26}$ theorem is an equivalent of the Hohenberg-Kohn theorem for time-dependent electron densities. It can be shown that there is a one-to-one mapping between the time-dependent external potential $v(\mathbf{r}, t)$ and the density $\rho(\mathbf{r}, t)$, up to an additive coordinate independent term $c(t)$ in $v(\mathbf{r}, t)$, under the condition that $v(\mathbf{r}, t)$ can be expressed by a Taylor expansion around $t=t_{0}$. The time-dependent Kohn-Sham equation can then be written as:

$$
i \frac{\partial \phi_{j}(\mathbf{r}, t)}{\partial t}=\left[-\frac{\nabla^{2}}{2}+v_{K S}[\rho(\mathbf{r}, t)]\right] \phi_{j}(\mathbf{r}, t)
$$

with the density

$$
\rho(\mathbf{r}, t)=\sum_{i=1}^{N}\left|\phi_{i}(\mathbf{r}, t)\right|^{2}
$$

and the potential

$$
v_{K S}(\mathbf{r}, t)=v_{\text {External }}(\mathbf{r}, t)+\int d^{3} \mathbf{r}^{\prime} \frac{\rho(\mathbf{r}, t)}{\left|\mathbf{r}-\mathbf{r}^{\prime}\right|}+v_{X C}(\mathbf{r}, t)
$$

where the integral term corresponds to the Hartree potential, from which it follows that the time-dependent exchange-correlation functional is dependent on electron density $\rho(\mathbf{r}, t)$ at all previous points in time. However, one can simplify matters by assuming the adiabatic approximation which takes $v_{X C}[\rho](\mathbf{r}, t)=\left.v_{X C}^{G S}[\rho]\right|_{\rho=\rho(\mathbf{r}, t)}$.

In the simplest case the the time-dependent part of the external potential is just a small perturbation of the system, causing a linear response in the electron density change, or in another words the time-dependent density $\rho(\mathbf{r}, t)$ depends linearly on the small perturbation part of the external potential. This is the basic assumption of the linear response theory which facilitates obtaining the excitation energies of a system from the time-dependent Kohn-Sham equations (TDKS). The system is perturbed by a weak field at $t=0$, the TDKS equations are propagated while evaluating a linear density-density response function $\chi[\rho](\mathbf{r}, t)$, the Fourier 
transformation of which gives the optical absorption spectrum. A small perturbation would cause a small difference in electron density $\rho(\mathbf{r}, t)=\rho_{G S}(\mathbf{r}, t)+\delta \rho(\mathbf{r}, t)$. A point-wise susceptibility $\chi\left[\rho_{G S}\right]\left(\mathbf{r}, \mathbf{r}^{\prime}, t-t^{\prime}\right)$ response of the ground state to the small perturbation can be written as:

$$
\begin{aligned}
& \chi[\rho]\left(\mathbf{r}, \mathbf{r}^{\prime}, \omega\right)=\chi\left[\rho_{K S}\right]\left(\mathbf{r}, \mathbf{r}^{\prime}, \omega\right) \\
& \quad+\int d^{3} r_{1} \int d^{3} r_{2} \chi\left[\rho_{K S}\right]\left(\mathbf{r}, \mathbf{r}_{1}, \omega\right)\left\{\frac{1}{\left|\mathbf{r}_{1}-\mathbf{r}_{\mathbf{2}}\right|}+f_{x c}\left(\mathbf{r}_{1}, \mathbf{r}_{2}, \omega\right)\right\} \chi[\rho]\left(\mathbf{r}_{\mathbf{2}}, \mathbf{r}^{\prime}, \omega\right)
\end{aligned}
$$

with the so-called exchange-correlation kernel

$$
f_{x c}\left[\rho_{G S}\right]\left(\mathbf{r}, \mathbf{r}^{\prime}, t-t^{\prime}\right)=\left.\frac{\delta v_{x c}(\mathbf{r}, t)}{\delta \rho\left(\mathbf{r}^{\prime}, t^{\prime}\right)}\right|_{\rho=\rho_{G S}}
$$

The response function $\chi$ has poles at the transition frequencies of the system which correspond to electronic excitations.

In the case of frequency independent kernels, the search for poles of $\chi[\rho]\left(\mathbf{r}, \mathbf{r}^{\prime}, \omega\right)$ is equivalent to the eigenvalue problem 27 :

$$
\sum_{q^{\prime}} R_{q q^{\prime}} F_{q}^{\prime}=\Omega_{q}^{2} F_{q}
$$

with $R_{q q^{\prime}}=\omega_{q}^{4} \delta_{q q^{\prime}}+4 \sqrt{\omega_{q} \omega_{q^{\prime}}} K_{q q^{\prime}}$ where $K_{q q^{\prime}}=\int d^{3} r \int d^{3} r \xi_{q}^{*}(\mathbf{r}) f_{H x c}\left(\mathbf{r}, \mathbf{r}^{\prime}\right) \xi_{q^{\prime}}$ and the Hartree-exchange-correlation kernel $f_{H x c}\left(\mathbf{r}, \mathbf{r}^{\prime}\right)=1 /\left|\mathbf{r}-\mathbf{r}^{\prime}\right|+f_{x c}\left(\mathbf{r}, \mathbf{r}^{\prime}\right)$. The lowest eigenvalues give the excitation energies $\Omega$. Since there exist efficient algorithms to find only the lowest excitation energies from these equations this is the form of TDDFT most widely implemented in quantum chemical program packages.

\subsubsection{Strong Fields: TDDFT Beyond Linear Response}

The solution to the TDKS Eq. 2.63 gives the exact density of the system without any constrains on the external potential. Let us cunsider the potential $v_{t d}(\mathbf{r}, t)=$ $E f(t) z \sin (\omega t)$ due to a laser field with frequency $\omega$ intensity $E$ and pulse shape $f(t)$ that acts on a system in its ground state. If the field is strong, it requires stepping beyond the linear approximation of the density-density response function by integrating the TDKS in time explicitly 28 . 
From the Runge-Gross theorem (see 2.3.2 follows that even non-linear timedependent behavior of a system, for example the ionization probabilities, must be described by a functional of the time-dependent density $\rho(\mathbf{r}, t)$. Although these functionals are not known, there are approximate methods of handling the ionization of a system in strong fields. A space of bound states can be defined which corresponds to the space where the electron density of the system is confined before the interaction with the strong external field. During the interaction, the density which crosses the boundary of this region accounts for the ionization processes.

\subsection{Nuclear Wavepacket Dynamics}

In a wide range of problems the molecular dynamics of a system is assessed in simple classical terms, the most common reason being a very high number of atoms resp. molecules taking part in the dynamics of the studied system. The classical approach is based on the Newton's equation of motion $\mathbf{F}_{\mathbf{i}}=m_{i} \mathbf{a}_{\mathbf{i}}$. Depending on the total size of the system (the number of atoms), the molecular system is divided into mass subunits $i$ which can be either single atoms or molecular fragments with the mass $m_{i}$, acceleration $\mathbf{a}_{\mathbf{i}}$ which move on the potential energy surface due to the forces $\mathbf{F}_{\mathbf{i}}$.

A number of numerical methods of solving the equation of motion exists, Verlet and velocity Verlet algorithm, leap-frog algorithm and Beeman's algorithm just to mention a few. ${ }^{29|30| 31 \mid 32}$ In the Verlet algorithm the on-the-fly integration proceeds according to the prescription:

$$
\mathbf{r}(t+\delta t)=2 \mathbf{r}(t)-\mathbf{r}(t-\delta t)+\mathbf{a}(t) \delta t^{2}
$$

which is derived from the expressions for the position $\mathbf{r}$ at time points $(t+\delta t)$ and $(t-\delta t)$ with the acceleration $\mathbf{a}$. This algorithm is very simple and straightforward. It can be supplemented by the velocity Verlet algorithm in which both the positions and velocities are obtained at every step.

When dealing with relatively small molecular systems however, the motion of the nuclei can be treated quantum-mechanically. Generally one has two options for solving the time-dependent Schrödinger equation for the nuclear degrees of freedom. The first one is based on the knowledge of the complete set of eigenstates 
of the time-independent Schrödinger equation from which one obtains the timedependent solutions as:

$$
\Psi(\mathbf{r}, t)=\sum_{j=1}^{N} c_{j} \exp \left(-i \frac{E_{j} t}{\hbar}\right) \Psi_{j}(\mathbf{r})
$$

where $\sum_{j=1}^{N} c_{j} \Psi_{j}(\mathbf{r})$ denotes the nuclei wave-function of the system at $t=0$ expanded in the set of eigenfunctions $\Psi_{j}(\mathbf{r})$ of the Hamiltonian with the eigenenergies $E_{j}$. In most cases however the system is too complicated to allow the knowledge of the complete set of eigenvalues and one has to resort to the direct integration of the time-dependent Schrödinger equation.

The wave-packet propagation in time draws on the benefits of expressing the wave-packet problem on a coordinate-space grid. In this basis the potential part of the Hamiltonian is diagonal while the coupling between the terms stems from the kinetic energy operator. However, the kinetic energy operator is straightforward to deal with for example in terms of fast Fourier transformation (FFT) described in the following (for a review see [33]).

In the FFT method of dealing with the kinetic energy the wave function is expressed through its Fourier transformation:

$$
\Psi\left(x_{j}\right)=\sqrt{\frac{1}{N}} \sum_{k=-N / 2}^{k=N / 2} c_{k} \exp (-i 2 \pi k j / N)
$$

where $j=0,1, \ldots, N$ is the number of grid points, $x_{j}=j \Delta x+0.5 \Delta x$ and $\Delta x=$ $\left(x_{\max }-x_{\min }\right) / N$. The kinetic energy will thus have a simple form in the Fourier space based on the general form:

$$
\frac{\partial^{n} \Psi(x)}{\partial x^{n}}=\sqrt{\frac{1}{N}} \sum_{k} c_{k}\left(-\frac{i 2 \pi k}{N \Delta x}\right)^{n} \exp (-i 2 \pi k j / N)
$$

with a trivial reverse transformation. Although the FFT method is faster, it is sometimes beneficial to use the so called discrete variable representation method, which is based on interpolating a function between the grid points. The advantage of this method is that the interpolation functions can be chosen to fulfill specific boundary conditions of a given problem.

The time propagation of the wave-packet can be also expressed in a number of 
methods. The simplest scheme is the so called second order difference scheme:

$$
\Phi\left(x_{n}, t+\Delta t\right)=\Phi\left(x_{n}, t-\Delta t\right)+\frac{2 \Delta t}{i \hbar} \hat{H} \Phi\left(x_{n}, t\right)
$$

It requires small steps $\Delta t$ due to its error $\sim(\Delta t)^{3}$.

One of the popular and stable methods is the split operator method. In this scheme one uses that:

$$
\begin{aligned}
\Phi\left(x_{n}, t+\Delta T\right) & =\exp \left(-\frac{i \Delta t \hat{H}}{\hbar}\right) \Phi\left(x_{n}, t\right) \\
& \approx \exp \left(\frac{i \hbar \nabla^{2} \Delta t}{4 m}\right) \exp \left(-\frac{i V \Delta t}{\hbar}\right) \exp \left(\frac{i \hbar \nabla^{2} \Delta t}{4 m}\right) \Phi\left(x_{n}, t\right)
\end{aligned}
$$

This method is a third order method in $\Delta t$ due to the splitting of kinetic energy operator. In order to evaluate the exponential operators either the FFT or DVR method can be used. In the case of FFT, using Eq. 2.72, one gets:

$$
\exp \left(\frac{i \hbar \nabla^{2} \Delta t}{2 m}\right) \Phi\left(x_{j}\right)=\frac{1}{\sqrt{N}} \sum_{k} c_{k} \exp \left[\frac{i \hbar \Delta t}{2 m}\left(-\frac{i 2 \pi k}{N \Delta x}\right)^{2}\right] \exp \left(-\frac{i 2 \pi k j}{N}\right)
$$

An advantage of the split operator method is that it is unitary, and therefore preserves normalization of the wave function at every time-step.

There is a number of other time-propagation methods. One of the methods capable of taking larger time-steps and also convenient to use in case of timeindependent Hamiltonians, is the so called Chebyshev method. ${ }^{34}$ In the case of time-dependent Hamiltonians the method of choice is the so called Lanczos reduction technique. $\frac{35}{35}$ 


\section{Chapter 3}

\section{X-ray Scattering and Photoisomerization of Stilbene}

\subsection{Introduction}

In the Section 2.2 we derived a general theoretical outline for calculating timedependent atom-atom correlation functions from first principles. In this formalism for the description of such correlation functions and their dependencies one can show their proportionality to the molecular form factors and therefore to quantities obtained from time-resolved x-ray scattering experiments. This formalism is in this Chapter applied to the photoisomerization of stilbene. The wide-angle $\mathrm{x}-$ ray scattering signal is calculated directly from electron densities as obtained from quantum-chemical calculations. The x-ray scattering techniques can provide additional information about molecular processes to the information obtained employing spectroscopic methods, since x-ray scattering probes the electron density which theoretically describes a system in its ground state completely. Wide-angle x-ray scattering offers a possibility to study electron densities in non-periodic complex systems, which renders it a suitable technique for the investigation of (bio)organic systems.

In recent years, as the experimental challenges have been overcome, in biol- 
ogy and chemistry time-resolved x-ray diffraction and x-ray scattering have gained in importance in the field of structural change characterization and dynamics of photo-activated molecular systems. The technical achievements at today's synchrotron sources (synchronization of ultrafast lasers to synchrotrons, enhancement of the x-ray flux, high repetition frequency of the experiment) opened the possibility to investigate structural changes of weakly scattering matter like liquids and to study their structural responses upon photo-excitation up to a time resolution

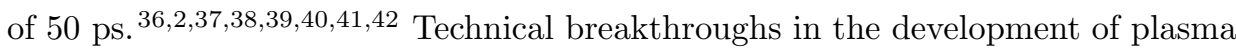
sources for the generation of x-rays make it possible to study ultrafast structural dynamics of condensed matter with periodical order. ${ }^{43 / 44 / 45 / 46}$ As the technical improvements move forward, $\underline{47}$ and planned $\mathrm{x}$-ray sources like the $\mathrm{x}$-ray free electron laser (XFEL) or high-repetition rate sources like the energy recovery linac (ERL) come to a stage of building-up,, $48 / 4950$ new scientific questions have to be raised and answered, also from a theoretical point of view.

One of the questions which automatically arise and which has to be answered is to what extend a quantum chemical treatment is required in order to describe the dynamics of ultrafast processes from an x-ray scattering point of view. In ultrafast spectroscopy, the concept of wave-packet dynamics has been successfully applied to the description of photo-induced ultrafast processes in molecular systems. Non-adiabatic transitions between the potential surfaces ${ }^{51}$ as well as vibrational and/or rotational energy redistribution and relaxation processes can be expressed in a wave-packet propagation scheme leading to a description of ultrafast coherent molecular motions, dephasing and energy dissipation into rovibrational eigenstates of the system. Even the control of wave packet motion by focusing the delocalized wave packet has been proposed ${ }^{52}$ and experimentally proved ${ }^{53}$. In time-resolved x-ray scattering experiments one observes the changes of scattered $\mathrm{x}$-ray intensities as a function of time. Since the scattered x-ray intensities can be described as Fourier transformations (FT) of electron densities, the time-evolution of x-ray intensities can also be described in an electron density time-propagation scheme. In this Chapter we apply the description of electron density changes which is based on a full quantum-chemical approach from first principles.

Obtaining the electron density distribution of an electronic system from quantumchemical methods is theoretically straightforward and as such the electron density has been suggested as a candidate for comparison of theoretical predictions and 
experiments 5455 especially while studying the ground-state structure of the system under consideration. The electron density is experimentally obtained most commonly from x-ray diffraction methods. The methods practically used for the electron density analysis are based on the comparison between calculated and experimentally determined electron densities. By applying Laplacian analysis to the electron density distributions, and partitioning, chemical bonding and their changes could be characterized. $\frac{55}{5}$ The relation between x-ray charge densities and chemical bonding has been summarized by Coppens. $\frac{56}{5}$

In this Chapter, we take a closer look at the description of x-ray scattering as it is theoretically derived from the electron density distribution. The wellknown relation between the electron density and the x-ray scattering signal can be used to directly calculate the scattering spectra. In the case of the electron density description in terms of Gaussian-type orbitals (GTOs) $)^{57}$ the calculation is analytically exact. Moreover, we will show how this approach can be easily used for studying time-dependent processes in electronic systems.

Atomic orbitals are best represented by Slater-type orbitals (STOs) which are similar to the exact analytical solution of the Schrödinger equation for hydrogenlike atoms. These functions include the cusp of the $s$-orbitals at the position of the nucleus which is responsible for the sharp peaks of the electron density at the atomic nuclei. For computational reasons, GTOs, introduced by Boys 57 and nowadays used in most common electronic structure programs, are much more suitable due to the considerably facilitated evaluation of four-center two-electron integrals.

The knowledge of the electron density of a particular electronic system is sufficient for the determination of the ground state energy of this system ${ }^{58}$ as well as some of its important properties. Experimental techniques measuring this quantity are the x-ray scattering methods. Quantum-mechanical treatment (e.g. by Fei ${ }^{599}$ ) shows that in the first Born approximation scattering of a radiation field on a molecule with electron density $\rho(\mathbf{r})$ leads to the well known dependence of the scattering intensity on the electron density which, normalized against the scattering intensity of one electron, reads:

$$
I=\left|\int d^{3} \mathbf{r} \rho(\mathbf{r}) \exp (-i \mathbf{k r})\right|^{2} .
$$


Here, $\mathbf{k}=\left(k_{x}, k_{y}, k_{z}\right)$ is the scattering vector.

A method of particular importance for studies of liquid samples, organic solutions or biological samples, is wide-angle x-ray scattering (WAXS). $\underline{60}$ The signal obtained in WAXS basically corresponds to the spherically averaged FT of the electron density of the studied system and naturally a large part of the information about the electron density is lost after this averaging. If we attempted to use this method for time-resolved studies, the experimental resolution would have to be very high in order to resolve all the information needed to describe the electron density changes in the system under investigation. The requirements on the experimental resolution depend on how detailed electron density changes we are interested to measure. In standard classical x-ray diffraction theory the atoms are modeled by a spherical distribution of electron density which is practically connected to the atomic scattering factor.

Since a liquid sample consists of the system (molecule) of interest, dissolved in a suitable solvent, we should shortly mention the effects of the solvent. The solvent should be chosen so that the signal from its structure is pronounced in the spectra as little as possible and in a non-disturbing way. In the case of timeresolved measurements the strongest signal from the solvent corresponds to solvent heating.

As a model process for our time-dependent study we chose the photoisomerization of stilbene (Fig. 3.2 as a typical case of a photo-induced process. Generally photo-induced processes are of utmost importance in biochemistry, practically they are being assessed in the growing field of organic electronics. Understanding these processes is essential for their successful application in the various branches of material science. As usually molecules containing a large number of atoms are involved, the potential energy surfaces are accordingly complex. Furthermore, in photophysics, processes prohibited to spectroscopic measurements occur, though the studies of the electron density resp. its time evolution are still attainable.

There are various reasons for choosing the photoisomerization of stilbene as a test process. There has been a large amount of experimental work $\frac{6162163164165166167168}{6}$ done on this system because of its prototypical photochemical character. The transition from the excited state is almost $100 \%$ non-radiative and the process is irreversible. From a theoretical point of view, it is a system of moderate size, and the number of atoms involved can be easily assessed by most of the conventional 
quantum-chemical methods. Indeed stilbene has been already extensively studied theoretically. $69 / 70 / 71$ The reason for the choice of this system for x-ray scattering measurements is also quite clear. The large amplitude motion from planar $180^{\circ}$ trans conformation to the $7^{\circ}$ cis conformation, where the angles refer to the central dihedral angle, means strong electron density changes. The signal from these changes is the most pronounced part of the scattering spectra. On top of this we will show the fine structure of signal changes stemming from small variations of the electron density due to bonding and delocalization.

Our goal is to estimate the possible application of x-ray scattering methods to the measurement of time-dependent processes. In Section 2.2 we recollect the theoretical aspects needed for assessing the problem.

We calculate the WAXS spectra from the FT of the electron densities as given by density functional theory (DFT) calculations and compare the results with the WAXS spectra obtained from the classical Debye equation. We show that the WAXS signal theoretically has a potential to show features such subtle in electron density delocalization as double bonds or delocalized electrons in phenyl rings though experimentally resolution of XFEL is needed to resolve these.

\subsection{Potential Energy Surface}

The calculations of the ground and excited state PESs of stilbene were performed using the Gaussian03 ${ }^{72}$ program package. The results can be seen in Figure 3.1. We employed DFT for the electronic ground state calculations, resp. timedependent DFT (TDDFT) for calculations of vertical excitation energies, using the B3LYP $\frac{73 \sqrt{23}}{\text { and B3P86 }} 6^{\sqrt[24 / 74]{74}}$ functionals as implemented in the Gaussian package. The choice of B3P86 can be supported by the fact that it was found to perform the best in calculating the excitation energies of ethene. $\frac{75}{\sqrt{75}}$ We use the $6-311++\mathrm{G}(\mathrm{d}, \mathrm{p})$ basis set in the calculation of the PES and the 6-31G(d) basis set in the calculation of the x-ray scattering.

The main geometry changes during the isomerization of the stilbene molecule (see Fig. 3.2 correspond to the dihedral angle $\alpha$ and the torsional angles of the phenyl rings $\beta$. The hydrogens at the central carbon atoms are also involved in the process. To avoid complications with the optimization of the geometries in the excited state we choose a simplified reaction path with an equidistant grid in the 


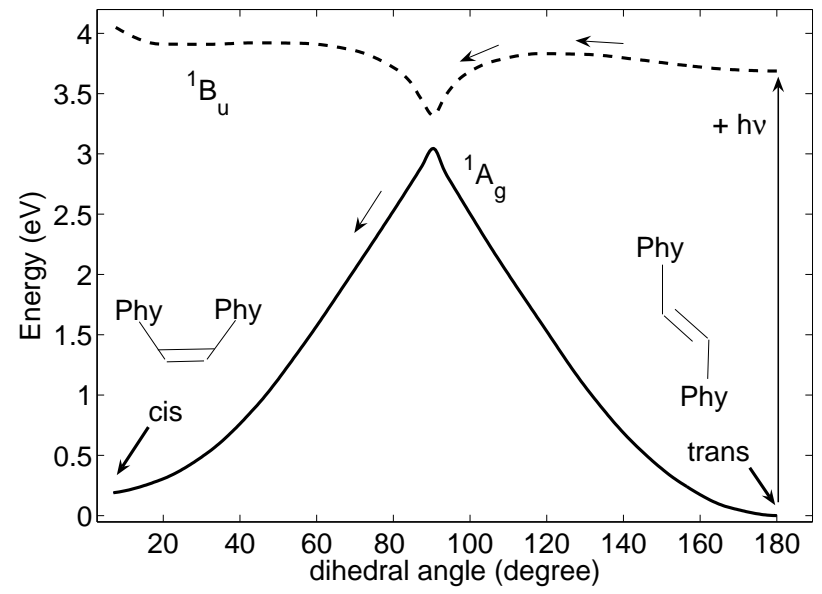

(a)

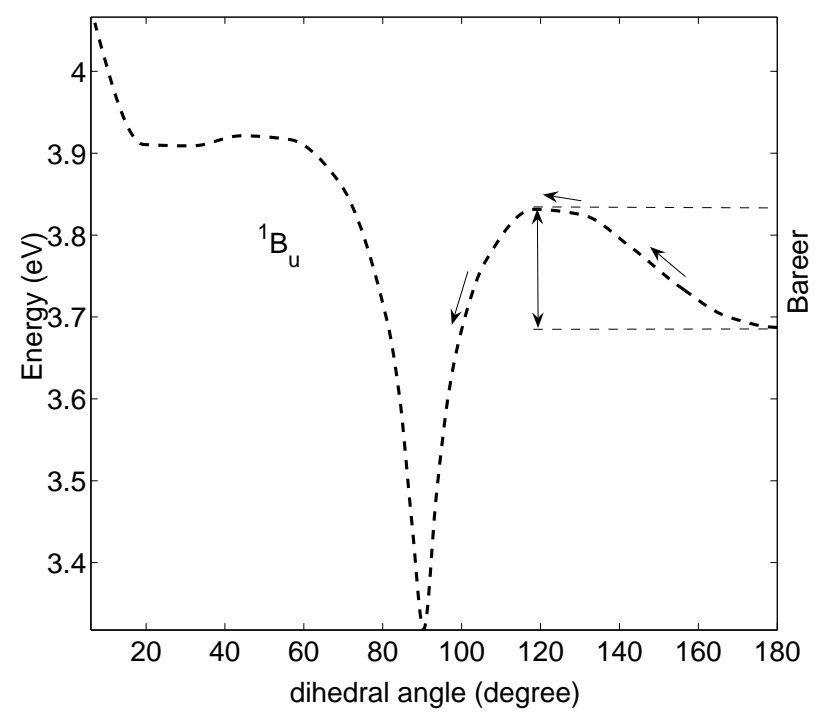

(b)

Figure 3.1. a) Potential energy surface of the ground state and the $S_{1}$ state. b) Zoom of the PES.

angles $\alpha, \beta$ and the angles of the central hydrogens from the optimized trans to the optimized cis geometry. The elongation of the central double bond as reported by Dietl et al. $\left[99\right.$ and Improta and Santoro $\frac{70}{}$ is also taken into account. We employ this simplification in order to obtain an approximate PES, bearing in mind that the 
detailed energetics of the process is not the aim of our studies. The small barrier (measured ${ }^{76}$ to be $1200 \mathrm{~cm}^{-1}$ and recently theoretically estimated $\frac{77}{70}$ to $750 \mathrm{~cm}^{-1}$ ) in the excited state between the Franck-Condon state and the $90^{\circ}$ configuration where the non-radiative transition over the conical intersection occurs ${ }^{77}$ is well reproduced (1197 $\mathrm{cm}^{-1}$ without correction for the zero point energy) even on the simplified reaction path chosen for our calculations.

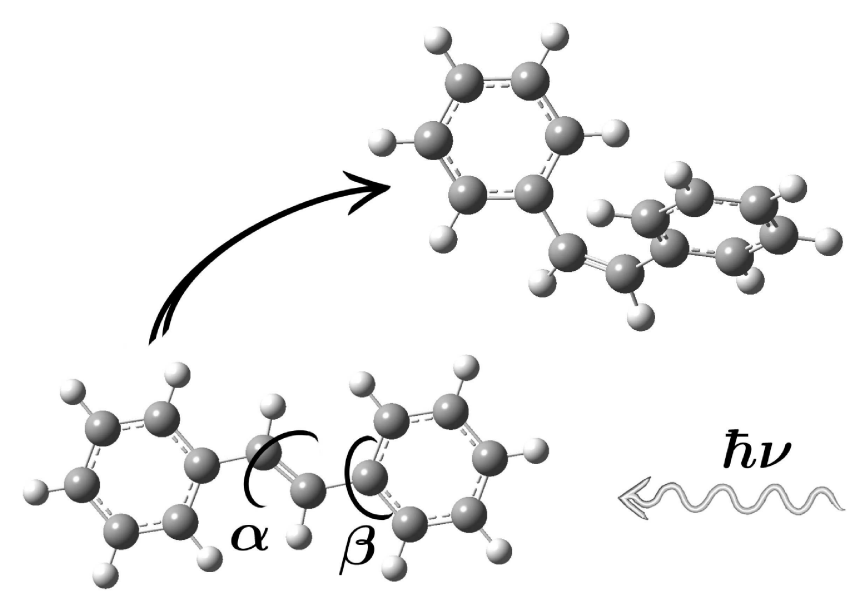

Figure 3.2. Sketch of the photoisomerization geometries (using GaussView 3.09 $9^{78}$ ). The main photoisomerization angles are the dihedral $\alpha$ which corresponds to the large amplitude motion and phenyl rings twisting angle $\beta$.

The conical intersection is the cause for difficulties in calculating the excited state reaction path. The TDDFT calculation has intrinsic limitations in this region (Ref. [70] approaches the intersection only to $20^{\circ}$ in the $\alpha$ torsional angle). A complete active space SCF (CASSCF) calculation approaching TDDFT in accuracy requires a large active orbital space ${ }^{71}$. But at this stage, our main reason for obtaining the reaction path is the following study of the isomerization dynamics in order to investigate the scattering signal differences as obtained from classical resp. quantum-mechanical calculations. Although approximate, the chosen reaction path is sufficient as a base for our further calculations where we take snapshots of the electron density along the reaction path by calculating the single molecule scattering signal. 


\subsection{The X-Ray Scattering Spectra}

Before integration, the $\left|\mathcal{F}_{\mathbf{k}}\{\rho(\mathbf{r}, t)\}\right|^{2}$ term in equation 2.46 corresponds to $\mathrm{x}$ ray scattering on one molecule. This is naturally much more sensitive to electron density changes than the WAXS signal which is averaged for random orientations of molecules. For a better insight into the problem, we will show the difference in the single molecule scattering from two different electron density distributions.

We start with a calculation of the signal change in the case of the smallest typical electron density change, namely HOMO-LUMO excitation of trans-stilbene. Figure 3.3 (a) shows the difference signal from the single molecule scattering of trans-stilbene in the ground electronic state and first excited Franck-Condon state. As we see, the maximum signal difference in this case is about $0.1 \%$ (given in $\%$ of the $k=0$ intensity, that is $\Delta I / I(k=0) \times 100)$. Stronger signal change can be expected if we remove one electron from a strongly localized core state (on one central carbon atom) and excite it into the LUMO orbital. Indeed the calculated absolute difference of the scattering signal amounts to $0.6 \%$ (Fig. 3.3 (b)).

The differences in the single-molecule scattering signal that come from electron density changes caused by changes in orbital occupancies are only subtle. We can compare them with the single-molecule scattering differences due to large geometry changes. Fig. 3.4 (a) represents the difference in the signal from trans and cis isomers, Fig. 3.4 (b) the difference in the signal from trans and $130^{\circ}$ dihedral angle geometry. The absolute difference (when normalized to $\Delta I / I(k=0) \times 100$ which means $\%$ of the $k=0$ intensity) is high (around 50\%) however, this is not as strongly pronounced in the WAXS spectra since the negative and positive contributions cancel each other.

Single-molecule scattering calculations present a motivation for further studies of scattering signals. When averaged over spherical angles they correspond to the WAXS signals. This averaging obviously leads to a loss of details in the spectra. The following results show what kind of effects are the WAXS spectra sensitive to. Our main aim here is to show the sensitivity to the detailed description of the electron density and later the sensitivity to the wave-packet description of the molecular degrees of freedom. We calculate WAXS signal changes as expected for the time-resolved scattering during the photoisomerization process. We take 'snapshots' of the molecule at different parts of the PES and compare the signal with the one obtained from the standard scattering formalism with atoms described by 


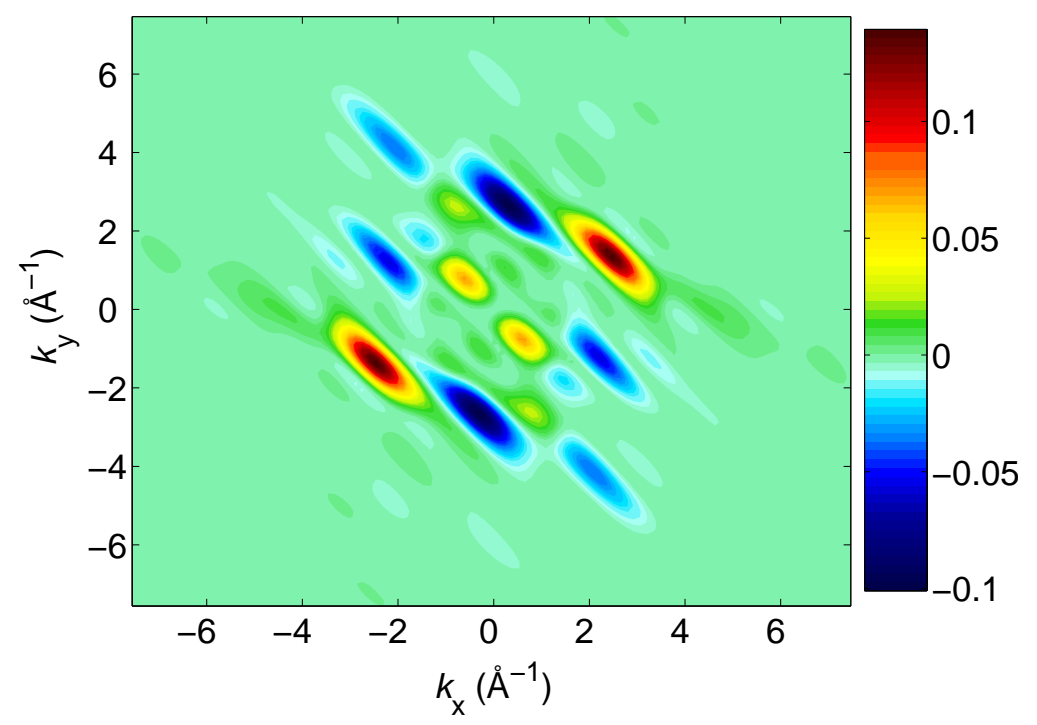

(a)

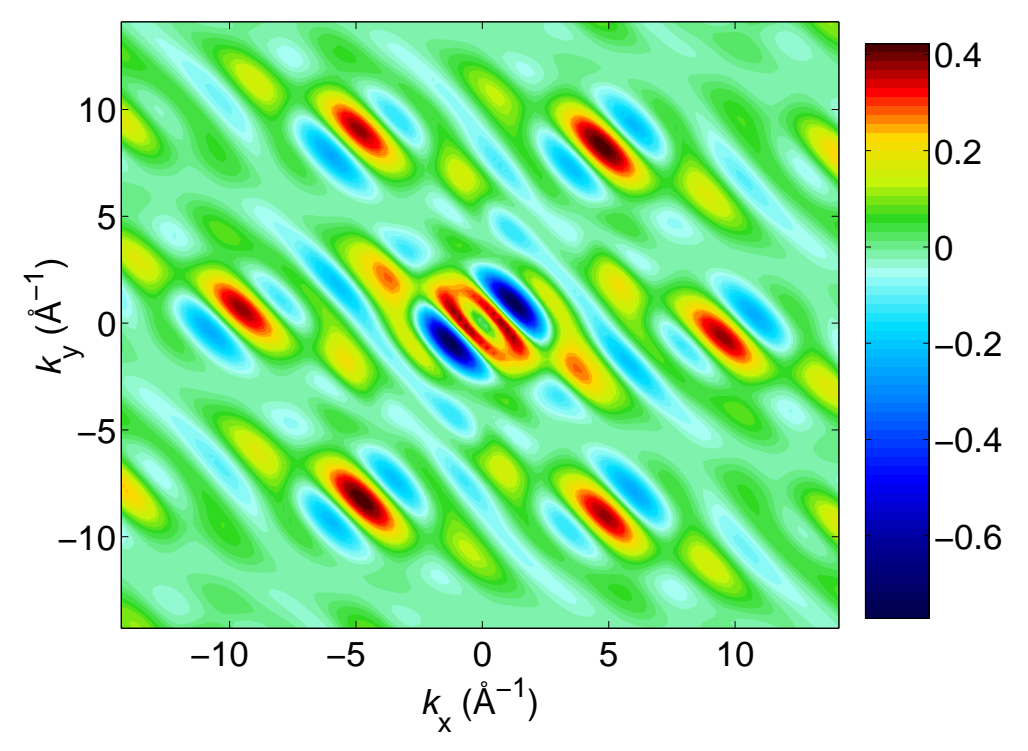

(b)

Figure 3.3. a) Scattering intensity difference of ground state and vertically excited state in $\%$ of $k=0$ intensity depicted on the color bar. A filled-contour graph. b) Scattering intensity difference of ground state and core excited state. For both we assume singlemolecule scattering on the trans stilbene. 


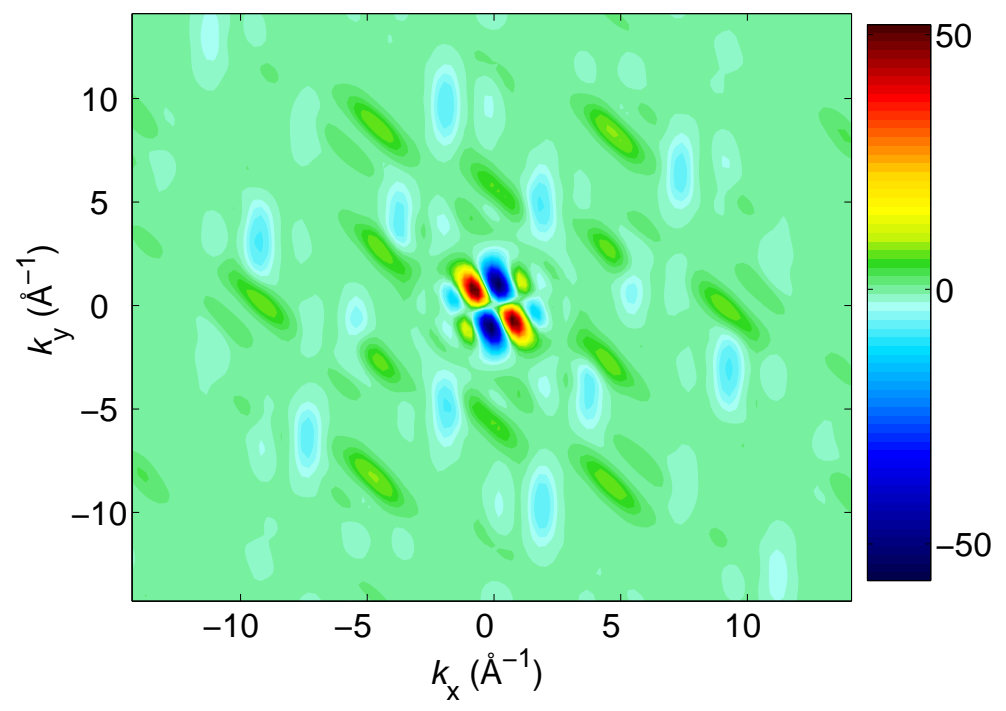

(a)

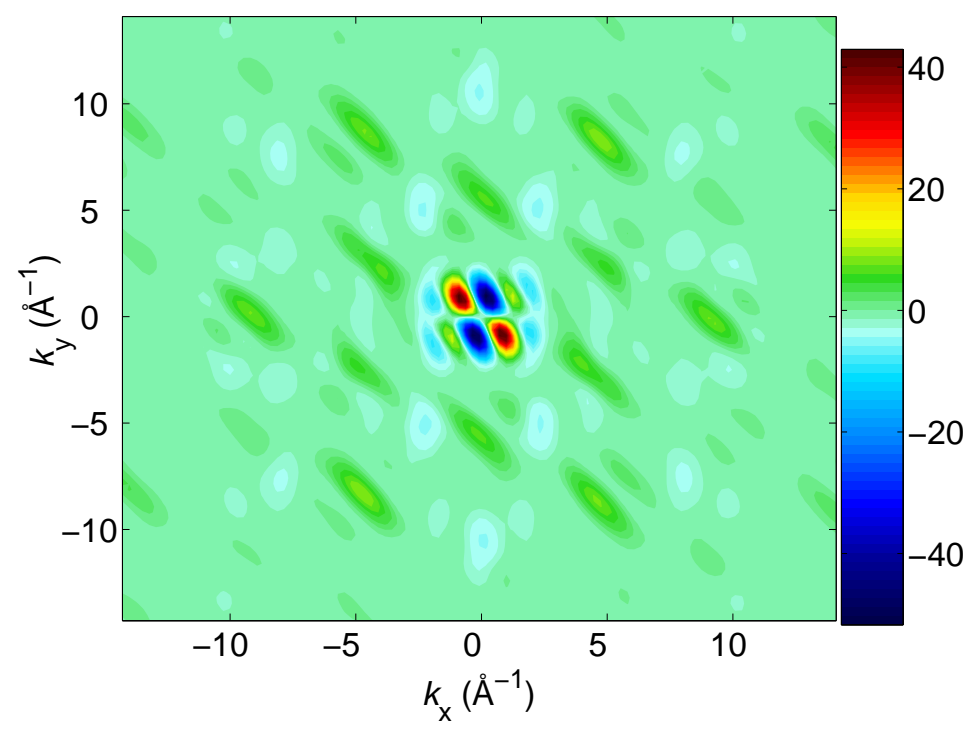

(b)

Figure 3.4. a) Scattering intensity difference of trans and $130^{\circ}$ state in $\%$ of $k=0$ intensity depicted on the color bar. A filled-contour graph. b) Scattering intensity difference of trans and cis state.

the atomic scattering factors in the Debye equation. These are further compared to the ones resulting from the wave-packet description of the nuclear degrees of 
freedom in the next section. By doing so we would like to examine if the WAXS method is suitable for time-resolved measurements.

In the following we call the difference in the WAXS intensity from two different molecular geometries at the same level of electron density assessment (Debye equation resp. detailed density approach) 'difference intensity'. As we can see from Fig. 3.5 (a) resp. from Fig. 3.5 (b) the trans to cis difference intensities as given by the Debye equation and direct Fourier transformation of electron densities are in a good agreement approximately up to $k=2 \AA^{-1}$. From here the difference between these two approaches starts to grow with higher $k$ It amounts to $0.3 \%$ at $k=3.1 \AA^{-1}$ (when normalized to $\Delta I / I(k=0) \times 100$ or $\%$ of the $k=0$ intensity), which in relative terms means that the Debye equation overestimates the difference signal by a factor of 3.3 at this $k$-point. At $k=5.2 \AA^{-1}$ it grows to $0.5 \%$ which means a factor of 9.3 overestimation by the Debye equation. We should point out that the region of higher $k$ values is the one where the effects of more localized changes in geometry become pronounced. The periodicity and the main features of the difference intensity remain the same.

If we would compare the original WAXS curves; Debye WAXS intensity and the detailed electron density intensity at the same geometry; they start to differ at $k \simeq$ $0.5 \AA^{-1}$ and the difference grows to approximately $3 \%$ at $k=3 \AA^{-1}$ as the signal from the detailed density decreases faster than the Debye signal. However, since the detailed electron density brings the similar delocalization to every geometry the WAXS signal from every geometry decreases in the same manner which causes the difference intensities from the detailed density to be approximately ten times smaller as compared to the Debye difference intensity.

\subsection{Dynamics}

In order to estimate the timescale of the process we have performed a classical simulation of the dynamics on the calculated PES, using the standard Verlet integration $^{29130}$ of the equation of motion. In the case of the photoisomerization of stilbene, this can offer only an order-of-magnitude guess about the timescale, since for full consideration the nonadiabaticity of the process must be assessed. Instead of taking into account the two state system we assume a simple path from the Franck-Condon state to the cis ground state over the conical intersection as if 


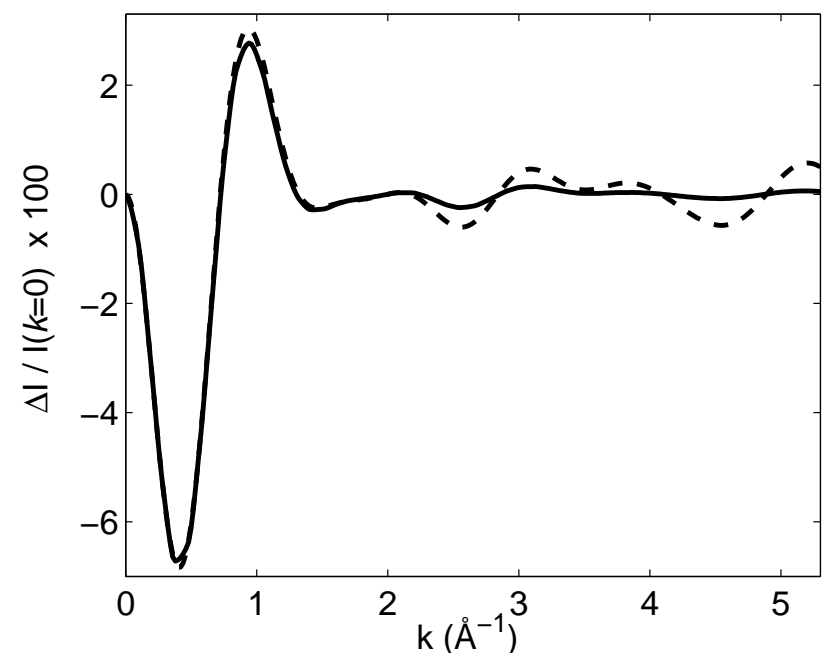

(a)

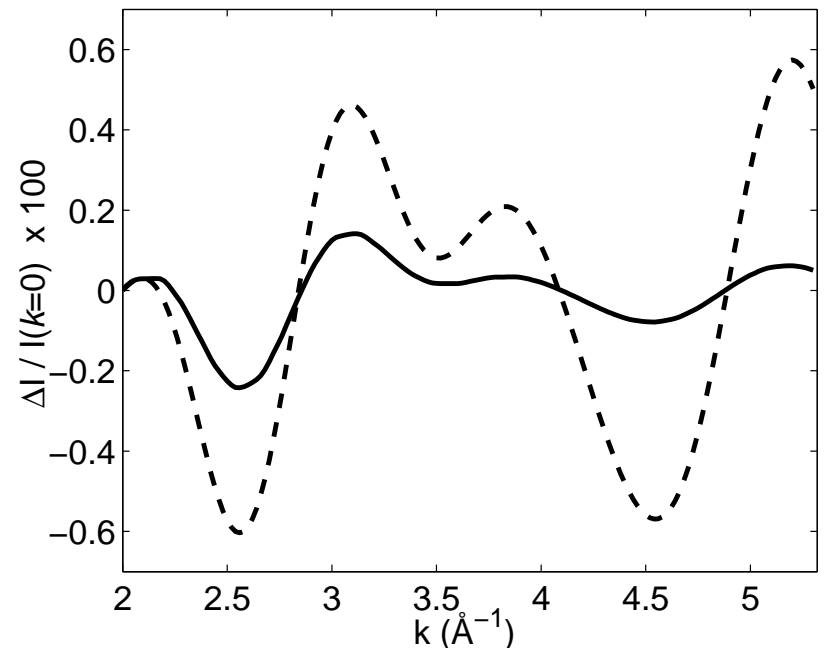

(b)

Figure 3.5. a) WAXS difference signal as given by Debye equation (dashed line) vs signal from the DFT/B3LYP electron densities (solid line). b) Detail of the WAXS difference signal at higher $k$-values.

it would belong to a single state. In the chosen internal coordinates described in the Section 3.2 the PES to a large extend changes smoothly and a small number of points is sufficient for the energy calculation. We perform the calculation in 22 points with higher density of points at the intersection and for the further calculations we increase the number of points with a simple quadratic spline on the 
obtained surface. We perform the Verlet integration on this approximate minimal energy photoisomerization path. In this way we approximate the timescale of the process to be on the order of picoseconds (about 2 ps, depending on the starting kinetic energy), with a fast relaxation from the first excited state to the ground state trough the conical intersection in the time scale on the order of femtoseconds (about $200 \mathrm{fs}$ ). The small starting kinetic energy used is just sufficient for the molecule to cross the small barrier in the excited state.

Some attention should be payed to the choice of the active coordinates. We have simplified the problem to one internal coordinate - the reaction path. This is by definition also the coordinate with the weakest confinement for vibrational motion. Including more than one dimension brings about problems in the integration according to Eq. 2.46 since the number of analytical functions needed for the description of the density of the wave-packet grows accordingly and the calculation becomes time-consuming.

In the following we present the studies on the influence of the wave-packet description of nuclear degrees of freedom on the WAXS signal and the difference intensities. Since at this stage we are not interested in a rigorous description of the isomerization reaction path, but rather in the possible effects which the wave-packet description can have on the scattering spectra, we will again employ significant simplifications. The calculation of the wave-packet dynamics on our simplified surface (as in the Verlet integration case) is performed in a standard split-operator procedure. ${ }^{[79}$ We include a small starting momentum to make the molecule move in one direction across the barrier. Figure 3.6 shows the first 250 fs of the photoisomerization process. The ground state wave-packet is strongly confined by the ground state potential, in the internal degree of freedom taken into account. As we could predict the WAXS signal of this well localized wave-packet is practically indistinguishable from the signal as coming from the trans single geometry electron distribution. After the vertical excitation $(t=0)$ the wave-packet quickly broadens on the shallow $\mathrm{S}_{1}$ surface. The wave-packet broadens during the isomerization process and is mostly much broader than the ground state wavepacket (see Fig. 3.6. In order to compare the classical and wave-packet approach to the time development of scattering spectra we calculate these spectra at different time points of the isomerization process. We choose the trans ground state, the wave packets in the first $60 \mathrm{fs}$ of the process and a later wave packet at $214 \mathrm{fs}$ 
the expectation value of which corresponds to a geometry with dihedral angle $\alpha=130.6^{\circ}$. The later wave-packet is chosen as one which corresponds to the excited state before the transition over the conical intersection because of our choice of the simplified reaction path. Note, that we have assumed circularly polarized light for the stilbene excitation which would generate a homogeneous distribution of the orientation of photoexcited chromophores. Under this assumption the dephasing effects for orientational distribution can be ignored.

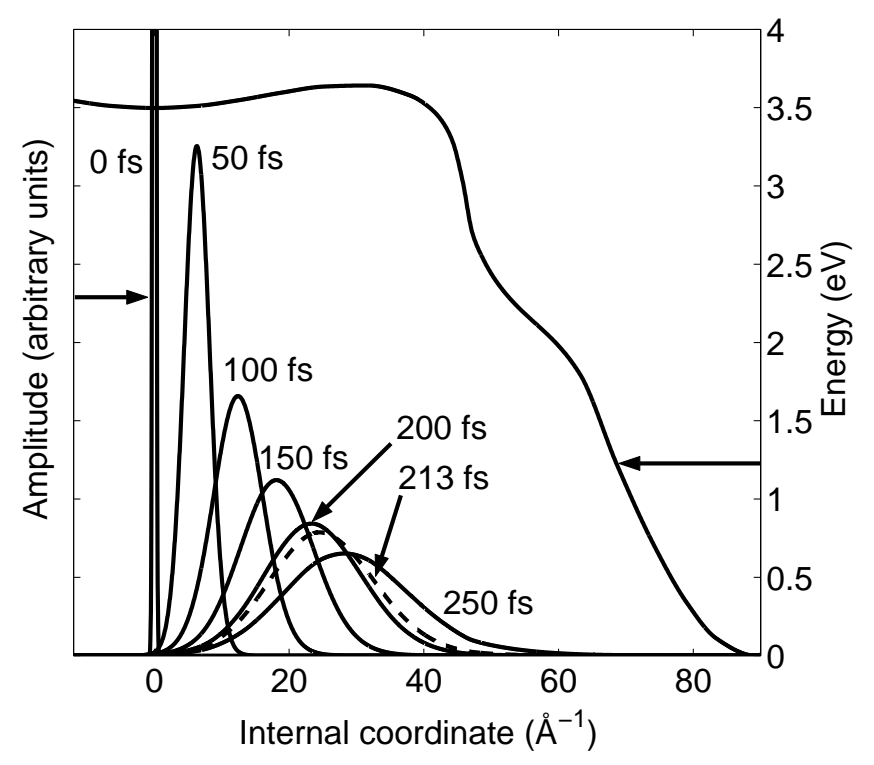

Figure 3.6. Wave-packet dynamics in the first $250 \mathrm{fs}$ of the photoisomerization. The PES used for in the dynamics calculation is shown for clarity. Both the PES and the wave-packet evolution are plotted against the internal mass weighted coordinate used in the calculation.

The x-ray scattering from a wave-packet can be treated in two different ways. We can assume times shorter than the internal vibrational redistribution decoherence time, which is expected to be about 50 to $100 \mathrm{fs}$. At this very short time scales the nuclear degrees of freedom are described as coherent wave-packets. The second case corresponds to times when the decoherence is pronounced in the system either due to the vibrational redistribution or interaction with the molecules from the environment like collisions. Here the wave-packet corresponds to a probability distribution of specific geometries in an ensemble of molecules.

Let us first consider the first, coherent case. The electron density of a system 
can be expressed as the expectation value:

$$
\rho(\mathbf{r}, t)=\langle\chi(\mathbf{R}, t)|\rho(\mathbf{r}, \mathbf{R}, t)| \chi(\mathbf{R}, t)\rangle
$$

where $\chi(\mathbf{R}, t)$ corresponds to the nuclear wave-packet with nuclear coordinates $\mathbf{R}$. In order to decouple the influence of the wave-packet description on the WAXS signal and the difference intensity we assume a simplified electron density distribution where the molecule is build solely from s-functions as in the Debye equation. This simple model density is easy to expand in nuclear wave functions, as described in Eq 3.2. In this way we get a delocalization in electron density due to the wave-packet description of the nuclear degrees of freedom. Figure 3.7 shows the difference intensities from the coherent wave-packets corresponding to $t_{0}(0 \mathrm{fs})-t(10,20,30,40,50,60 \mathrm{fs})$ and for comparison also the difference intensity coming from the ensemble wave packet description at times $t_{0}(0 \mathrm{fs})-t(10,60 \mathrm{fs})$. The decrease in WAXS intensity caused by the electron density delocalization in the coherent wave-packet is strong. As the wave packet broadens in time on the shallow $\mathrm{S}_{1}$ surfac the electron density delocalizes accordingly. The WAXS intensity

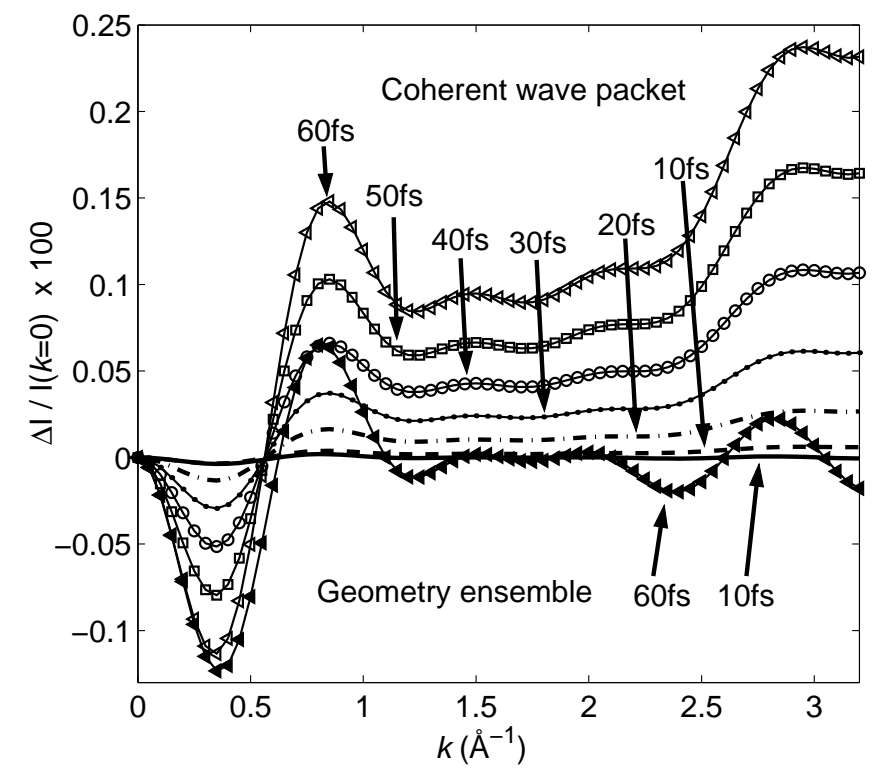

Figure 3.7. WAXS difference intensity as simulated from the wave-packet dynamics at $t=0-10,20,30,40,50,60$ fs coherent wave packets, compared to the intensity from an ensemble with geometry probabilities corresponding to the wave packet at $t=0-10,60 \mathrm{fs}$. 
from a broad wave packet is decreased in comparison to the WAXS intensity form a more localized wave packet at an earlier time point. The difference intensity $t_{0}-t$ then grows with increasing time $\mathrm{t}$, in particular at higher $k$ values since our time $t_{0}=0$ corresponds to the sharply localized Franck-Condon wave packet. The comparison with the corresponding difference intensities as calculated using the expectation geometry in the Debye equation and incoherent wave-packet described by probability distribution of the nuclear geometries is made in Figure 3.8. As we can see here the signal form the geometry ensemble shows only very small changes to the Debye equation difference signal at the expectation value of the particular wave packet for the whole $k$ range.

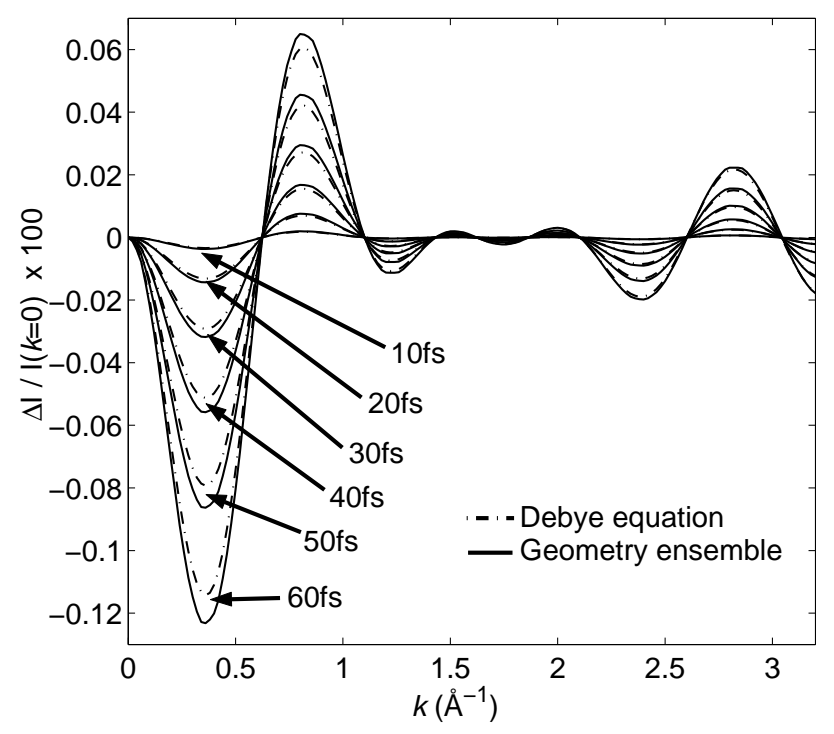

Figure 3.8. WAXS difference intensity as simulated from the Debye equation (solid line) at the expectation value of the nuclear geometry at $t=0-10,20,30,40,50,60 \mathrm{fs}$ coherent wave packets, compared to the intensity from an ensemble with geometry probabilities corresponding to the incoherent wave-packet at the same time points (dashed line)

After the coherence of the wave-packet is destroyed by molecular vibrations and interactions, the wave-packet corresponds to the incoherent probability distribution. For the sake of the test of the method we choose the wave-packet after 213 fs (with the expectation value corresponding to $130.6^{\circ}$ ) and calculate the WAXS signal using the Eq 2.46 with integration over the wave-packet geometry ensemble included. Although the WAXS signal from this kind of ensemble is not equal to the signal from the expectation value geometry, the calculated difference between 
them is in our case very small and no characteristic effect is observable, similarly to earlier time points of the isomerization process shown in Figure 3.8

\subsection{Conclusions}

As the experimental equipment gradually enables us to study liquid samples by WAXS measurements, it is important to reflect on the theory commonly used, in this case the Debye equation, which ignores the difference between the classical model of electron density and the more rigorous quantum chemical approach. As we have shown, this classical approach can be used only at small $k$ values (from 0 to approximately $2 \AA^{-1}$ ) which means the $k$ range where large-scale electron density effects are observable. In the range of $k$ values, where actual molecular geometry effects can be observed, electron delocalization becomes increasingly important and omitting quantum effects would lead to a misinterpretation of the spectra. In particular the Debye equation overestimates the sensitivity of WAXS which can be misleading when interpreting experimental data.

The time-dependent calculation of the scattering signal from the wave-packet dynamics has important implications. Assuming that trans-stilbene is in a low lying vibrational state (ground state in our case) the corresponding wave-packet can be considered as strongly localized. After a Franck-Condon excitation from this ground state the wave-packet broadens as it follows the reaction path. The broadening of the wave packet which corresponds to a vibrational redistribution in the excited state is observable on the further broadening of the scattering spectra. Evolution of the overall wave-packet in time is so observable on the scattering spectra. Both effects, the consideration of real electron density distribution and in the time-resolved case the consideration of nuclear degrees of freedom described by a wave-packet bring a considerable decrease of the intensity of the WAXS signal in particular at high $k$-values. However, in the case of the wave-packet calculations the dynamic interaction of x-rays with the molecule must be considered, which is a task for further studies. 


\section{Chapter 4}

\section{X-ray Scattering and Photodissociation of Iodine}

\subsection{Introduction}

The photodissociation and recombination of molecular iodine is a simple prototypical reaction, and as such it has been extensively experimentally studied. 80181182183184 Recently it has become an object for the development of time-resolved pump-probe x-ray diffraction experiments, measuring the time-scales of the process on the order of picoseconds. $\frac{85[86 / 87 / 88}{1 n}$ this Chapter we focus on detailed theoretical x-ray scattering studies of relevant excited states of molecular iodine and wave packet dynamics of the movements of nuclei during the photodissociation.

The motivation for the choice of molecular iodine as a model system for $\mathrm{x}$ ray scattering studies is straightforward. The high number of electrons means a strong scattering signal so that even an experiment with an $\mathrm{I}_{2}$ gas is possible. The photo-dissociation, triggered by 520nm laser pump pulse, can follow one of the two following paths. On the simpler first path, $\mathrm{I}_{2}$ is excited onto a dissociative energy surface. The second possibility is that $I_{2}$ is first excited onto an energy surface with a possible bound state but due to energy surface crossing it passes to a dissociative energy surface, $\frac{89}{20}$ as it can be seen on the Figure 4.1 a) between the energy levels B and $\mathrm{B}^{\prime \prime}$. The B state is also the most studied excited state in $\mathrm{I}_{2}$, as it is responsible for the visible absorption spectrum. The nature of the 
photodissociation reaction makes it a good choice for time resolved scattering experiments due to the dramatic change of bond the length, which has a strong effect on the x-ray scattering signal even when the studied system is an $\mathrm{I}_{2}$ solution.

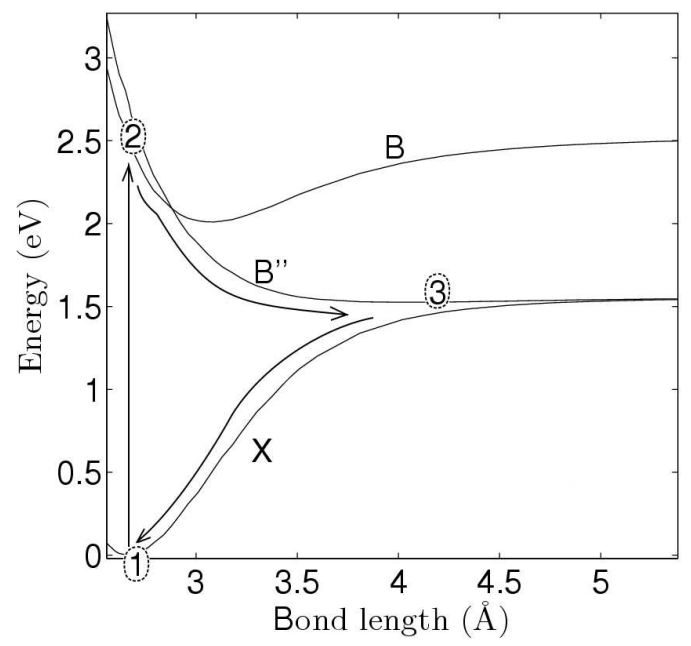

(a)

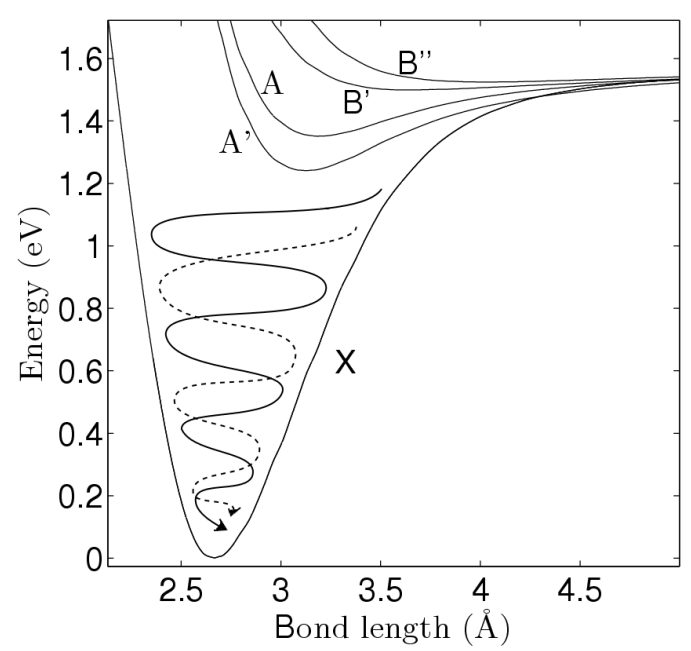

(b)

Figure 4.1. a) Potential energy surfaces of $I_{2}$. Schematically denoted are the photodissociation process $(1 \rightarrow 2 \rightarrow 3)$ and the relaxation to the ground state $(3 \rightarrow 1)$. b) Potential energy surfaces of $\mathrm{I}_{2}$ with the relaxation on the ground state denoted

The solvent plays an important part in the dissociation process, as it hinders the 
diffusion of the newly created iodine pair by absorbing the kinetic energy of the separated atoms 90 . This can lead to recombination of these same atoms, which is also called geminate recombination. This process happens on a time-scale on the order of $10^{2}$ picoseconds to nanoseconds. If the recombination ends with the molecule in the $\mathrm{A}$ or $\mathrm{A}^{\prime}$ state, the solvent further assists the relaxation into the grounds state over an energy level crossing. The time-scale of this relaxation process is strongly dependent on the solvent. $\underline{81 / 82}$ The geminate recombination is a faster process in comparison to recombination of tho iodine atoms that did not originate in the same $\mathrm{I}_{2}$ molecule. The time-scale of both of these processes has been the object of study of a number of publications using laser spectroscopy.

The determination of energy levels and properties of the iodine molecule $\mathrm{I}_{2}$ has been an object of several theoretical studies. $\frac{93|94| 95 \mid 96}{1}$ The main reason for the complicated energy levels of this seemingly simple molecule is the high nuclear charge of iodine, which leads to spin-orbit splitting of the electronic levels. The main electronic states studied in this work are depicted on the Figure 4.1 a) and $\mathrm{b})$. The state denoted as $\mathrm{B}$ is the ${ }^{3} \Pi_{u}\left(0_{u}^{+}\right)$state of the visible absorption spectrum. It dissociates into ${ }^{2} P_{3 / 2}+{ }^{2} P_{1 / 2}$ atomic states, while crossing repulsive or weakly bound states originating from ${ }^{2} P_{3 / 2}+{ }^{2} P_{3 / 2}$ atomic states. This

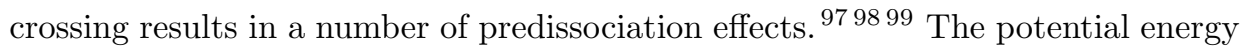
surfaces used for wave packet propagation calculations in this Chapter were taken from de Jong et al [100]. These PES were calculated using two different high level relativistic approaches for the ground and excited state calculations respectively. All-electron Dirac-Fock method followed by $\operatorname{CCSD}(\mathrm{T})$ calculations were applied in the case of the ground state and so include all relativistic effects from the outset. The excited states were calculated using average of configurations Dirac-HartreeFock approach followed by a complete active space CI calculation. The knowledge of the potential energy surface (PES) is required for the simulations of the the averaged wide angle x-ray scattering signal, and studies on the time evolution involving vibrational wave packet propagation.

Symmetric top molecules such as $\mathrm{I}_{2}$ are used also as test systems in x-ray scattering experiments. X-ray diffraction of aligned symmetric molecules has been studied on the example of $\mathrm{Br}_{2}$ by Ho and Santra in Reference [101. Here $\mathrm{Br}_{2}$ was chosen as a simple test system in order to study the influence of alignment and and laser field properties on the x-ray scattering spectra. 
One of the interesting implication of coherence in the case of vibrational molecular wave packets is the way it influences the total electron density of the sample in comparison to non-coherent wave packet. The coherence enhances the scattering signal and as such it is a good candidate for scattering studies of functional dependence of the electron density on time. Since the scattered x-ray intensities can be described as Fourier transformations (FTs) of electron densities, the time-evolution of x-ray intensities can also be described in an electron-density time-propagation scheme, as was described in the chapter 2.2 and applied on the example photoisomerization of stilbene in the Chapter 3.

\subsection{The $\mathrm{I}_{2} \mathrm{X}$-Ray Scattering Spectra and Excited States}

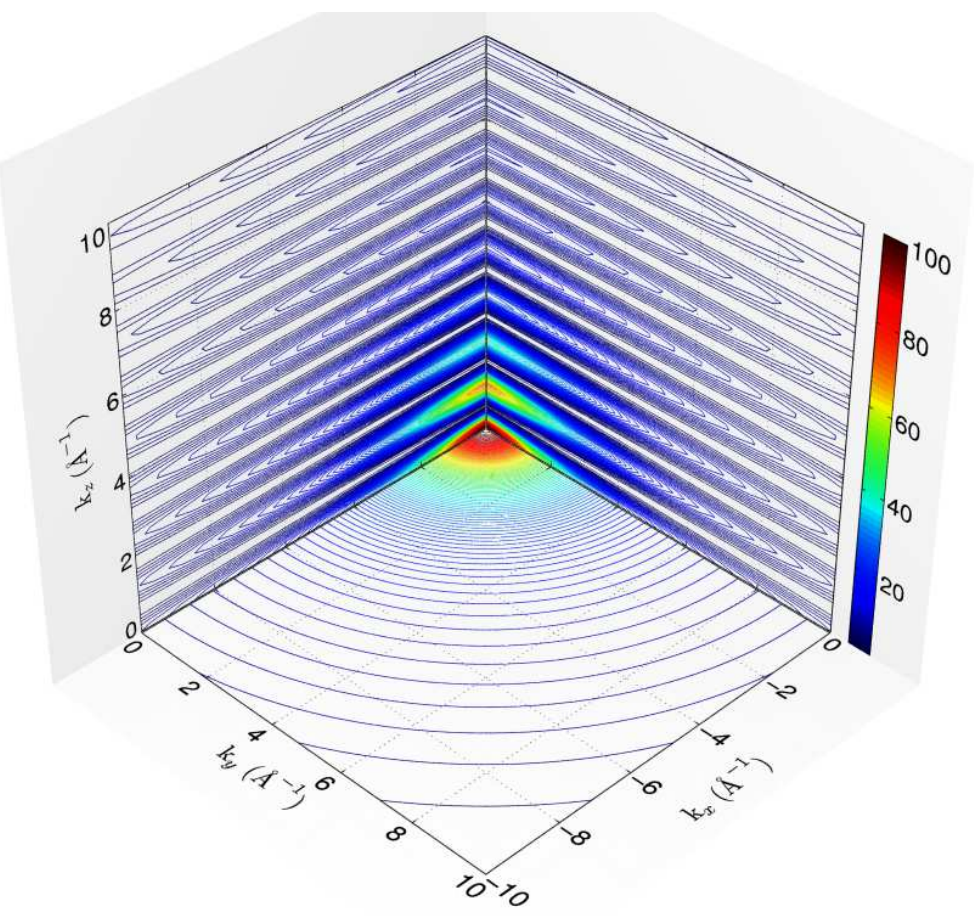

Figure 4.2. Total scattering intensity of $\mathrm{I}_{2}$. Color scale in $I / I(k=0)(\%)$

One of the reasons for choosing iodine for this study is the simplicity and high 


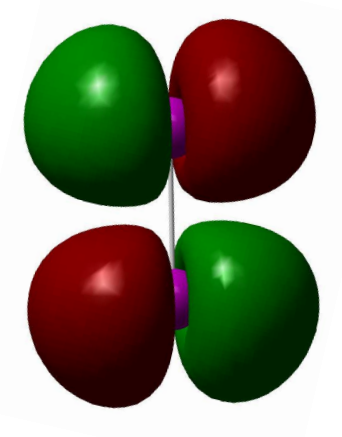

(a)

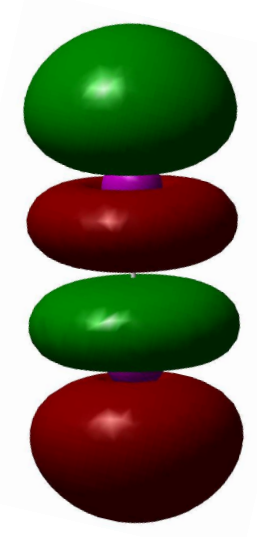

(b)

Figure 4.3. a) Highest occupied orbital ( $\Pi_{g}$ symmetry) of $I_{2}$ b) Lowest unoccupied orbital $\left(\Sigma_{u}\right.$ symmetry) of $\mathrm{I}_{2}$

symmetry of this molecule which allows focusing on a selected properties of the scattering spectra. One of the interesting effects is the influence of the electronic orbital structure on the scattering pattern. In order to observe the effect of such details as electron density change between different excited state one has to resort to the description of the scattering signal through the FT of detailed electron densities as it was describe in Section 2.2. In this scattering signal calculation the iodine molecule was described using the 6-31G(d) basis set at B3LYP level of theory. This would be insufficient for a high quality electronic calculation but it can easily quantify such subtle electron density changes that occur for example during vertical excitations. We start with the total scattering map of one iodine molecule as it is depicted in Figure 4.2 Most significant here are the fast changing maxima on the $\mathrm{k}_{z}$ axis representing the $\mathrm{I}_{2}$ the bond length. The $\mathrm{k}_{z}=0$ cut of the scattering intensity spectrum appears spherically symmetric due to the strong signal representing the core electrons density confined closely to the the nuclei.

One of the smallest theoretical orbital changes and consecutive electron density changes of a molecule is change represented by HOMO-LUMO excitation. In the previous Chapter we have shown that in the case of stilbene molecule this change causes a very small scattering signal change of only about $0.15 \%$. As we will demonstrate, in the case of iodine the difference between the ground state electron density and electron density of a vertically HOMO-LUMO excited state gives a 


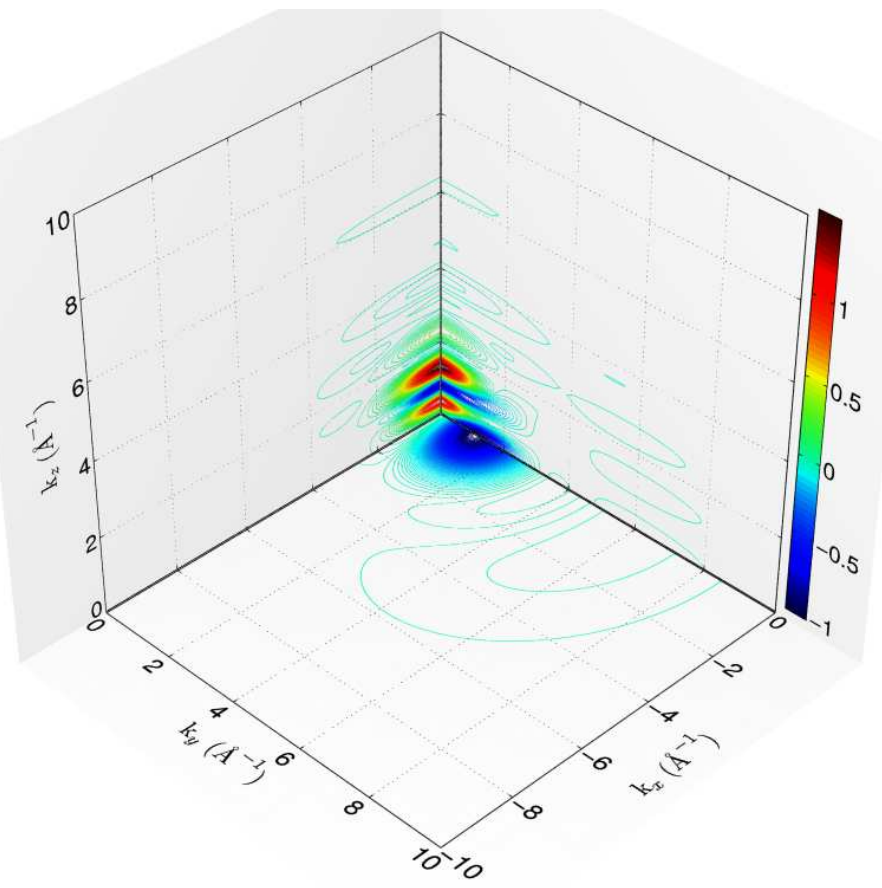

(a)

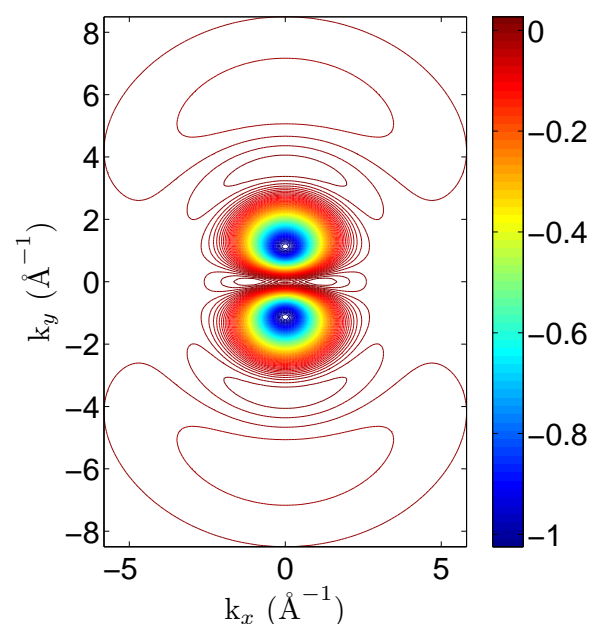

(b)

Figure 4.4. $\mathrm{I}_{2}$ scattering intensity difference in $\%$ of $k=0$ intensity. a) Intensity difference corresponding to the electron density change due to the HOMO-LUMO excitation from the ground state; b) The $\mathrm{k}_{z}=0$ part of the scattering intensity 


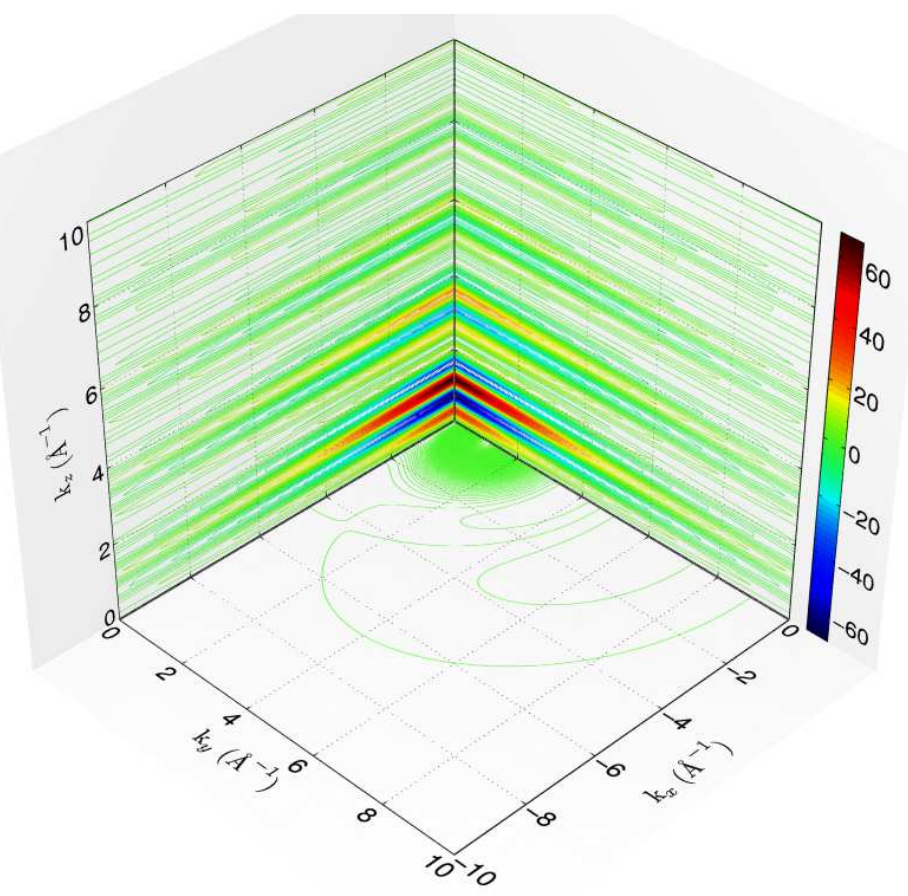

(a)

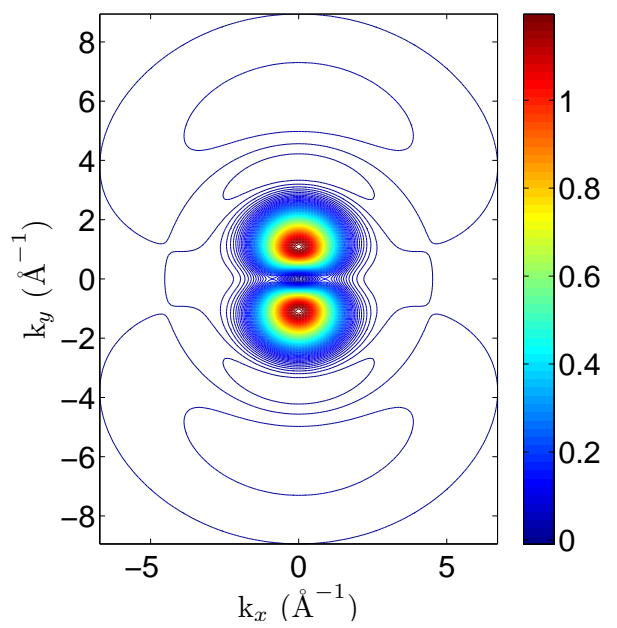

(b)

Figure 4.5. $\mathrm{I}_{2}$ scattering intensity difference in $\%$ of $k=0$ intensity. a) Intensity difference corresponding to the electron density difference between HOMO-LUMO with the bond length of $2.7 \AA$ and $\mathrm{I}_{2}$ during recombination with the bond length of $4.2 \AA$; b) The $\mathrm{k}_{z}=0$ part of the scattering intensity 


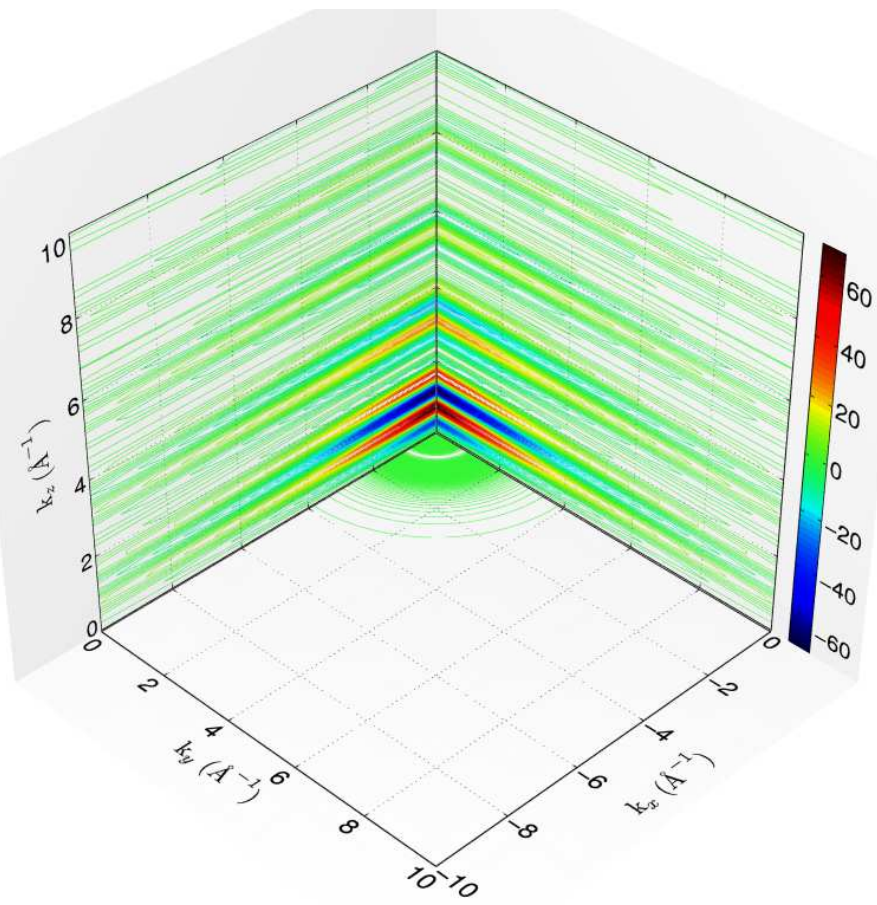

(a)

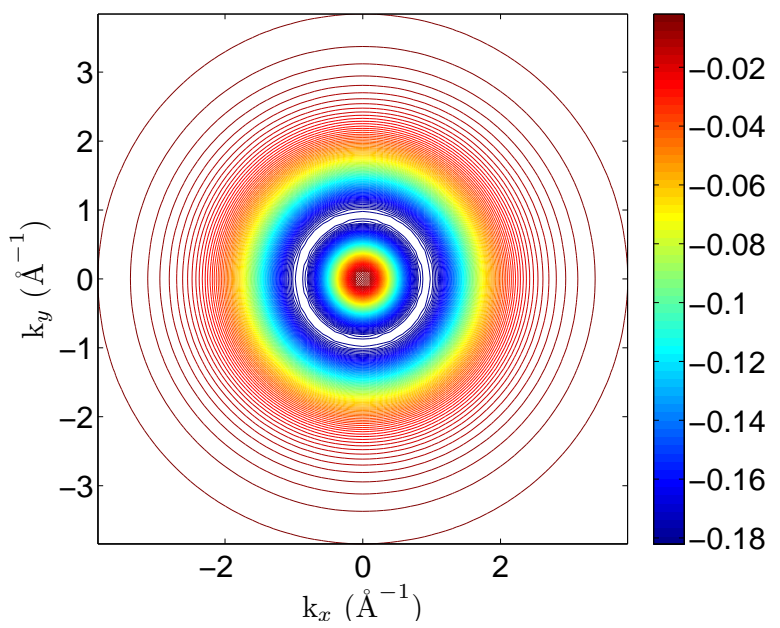

(b)

Figure 4.6. $\mathrm{I}_{2}$ scattering intensity difference in $\%$ of $k=0$ intensity. a) Intensity difference corresponding to the electron density change between ground states with two different bond lengths: $2.7 \AA$ and $4.2 \AA$; b) The $\mathrm{k}_{z}=0$ part of the scattering intensity 
much stronger signal in the scattering spectrum. The highest occupied and lowest unoccupied electronic orbitals of iodine in the ground state are depicted on the Figure 4.3 . Figure 4.4 shows the intensity difference between ground state and HOMO-LUMO excited state, or explicitly scattering intensity of an $\mathrm{I}_{2}$ molecule in the lowest excited state, with the bond length of $2.73 \AA$, minus the scattering intensity of an $\mathrm{I}_{2}$ molecule in its ground state. Therefore this intensity difference is caused purely by change in orbital occupancy rather than bond length change. In this way the negative values correspond to signal that is larger for the ground state structure while positive values correspond to signal that is stronger in the excited state intensity spectrum. As can be seen the symmetries of the difference scattering map correspond to the symmetries of structural difference. The main positive maxima, about $1.5 \%$ signal change, of this intensity difference are situated on the z-axis and correspond to the redistribution of electron density from the highest occupied orbital (see Fig. 4.3 a) to the lowest unoccupied orbital (Fig. 4.3 b).

In order to compare this still quite small intensity change we consider the dissociation of iodine. Figure 4.5 shows the intensity change when assuming a dissociation from the $\mathrm{A}^{\prime}$ state, the starting structure being the $\mathrm{I}_{2}$ molecule with the bond length of $2.73 \AA$ (which is the ground state bond length) with the orbital structure corresponding to the HOMO-LUMO excited state. The final structure corresponds to $\mathrm{I}_{2}$ in predissociation with the bond length of $4.2 \AA$ with the ground state orbital structure. The main maxima of the scattering signal, about $65 \%$, here correspond to the bond length change. However the signal corresponding to the change in the orbital structure can be seen on the $\mathrm{k}_{z}=0$ cut, being symmetrically similar to the HOMO-LUMO electron density scattering signal change.

Interesting is a comparison of the just mentioned case with the predissociation intensity difference corresponding to two ground state $(X)$ electron densities. This is depicted on the Figure 4.6. Although again the strongest signal naturally represents the bond length change, two additional observations can be made from this picture. First the electronic density does not undergo a symmetry change as in the previous case, which is observable from the spherically symmetric $\mathrm{k}_{z}=0$ cut of the scattering intensity spectrum. However it additionally shows another property. The picture has only negative values, showing two concentric minima of small absolute value (about $0.18 \%$ ). The intensity decrease here corresponds to the electron density that is tighter bound in the stretched molecule. 


\subsection{Observing $\mathrm{I}_{2}$ Dissociation with Time-Resolved X-Ray Scattering}

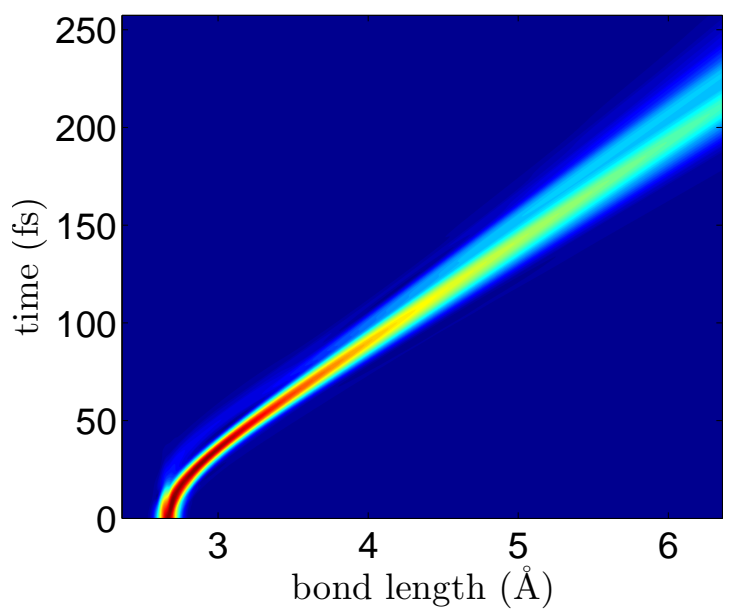

(a)

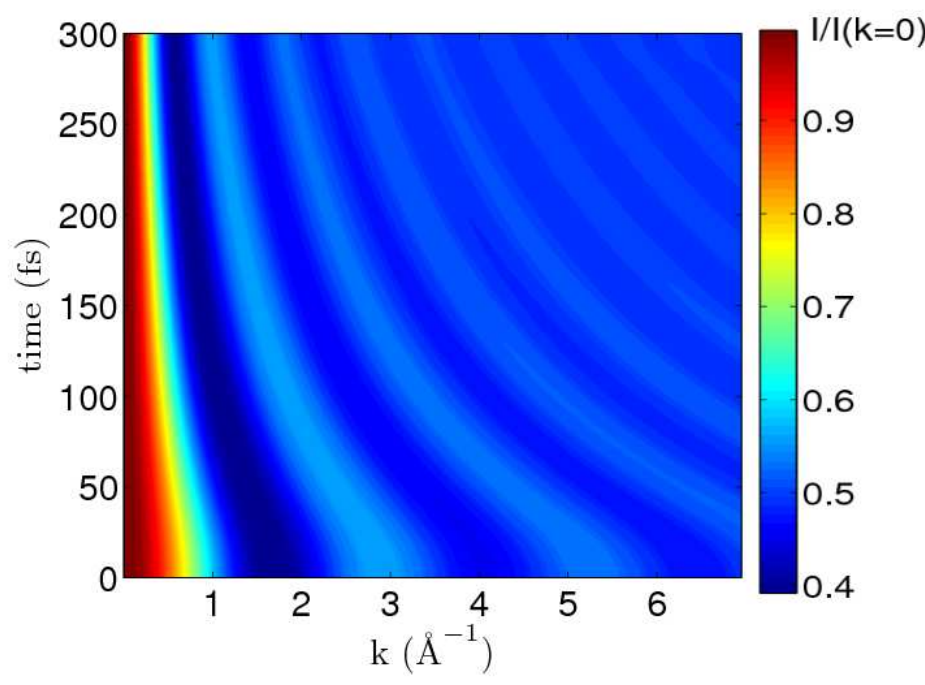

(b)

Figure 4.7. The dynamics of $I_{2}$ dissociation a) in real space (vibrational wave packet) b) $\mathrm{x}$-ray scattering signal (reciprocal space)

The dissociation of $\mathrm{I}_{2}$ is an example of chemical reaction especially suitable for study by x-ray scattering experiments since the scattering signal dramatically 


\subsection{Observing $\mathrm{I}_{2}$ Dissociation with Time-Resolved X-Ray Scattering 59}

changes with time up to the dissipation regime. In this case the simulated system in our calculations is represented by a randomly oriented ensemble of iodine molecules, what would be the case of liquid samples. Figure 4.7 shows the coherent part of the dissociation dynamics of iodine in real and reciprocal space. We use the split operator method to calculate the wave packet dynamics. It can be seen that the wave packet not only changes position with the proceeding dissociation, but also loses confinement. Reflecting this behavior the oscillations in the scattering spectrum become faster.

Figures 4.8 a) b) and c) and 4.9 a) and b) show the effect of pulse length on the scattering signal. It can be clearly seen that in the case of dissociation relatively long probe pulses give still quite a strong signal and time resolved studies should be possible for up to $\sim 100$ fs probe pulses. Here one has to consider the interaction between the $\mathrm{I}_{2}$ molecule and the solvent, since the dissipation process restricts the reliable time interval of the measurement.

At long time scales all that can be measured with the x-ray scattering is the thermal distribution of the vibrational modes on the ground state electronic surface as shown on the Figure 4.10. The long time scales we refer to here are time scales in which the system after dissipation achieved thermal equilibrium. A different number of vibrational modes are occupied corresponding to each temperature. The picture shows that although the number of occupied vibrational levels increases the change on the scattering intensity pattern is very small, on the order of tenths of percent. As expected, the higher temperatures contribute to the increase in the scattering signal in the higher k-range. The noncoherent thermal processes by their nature contribute to the diffuse signal and as such have only a small influence on the scattering signal of a randomly oriented molecular ensemble.

One gets a different picture while studying processes confined to a small area on the PES. To test this kind of process we study a fictional (meaning that we have no means of starting this particular collective coherent bouncing in reality) example of coherent bouncing of the iodine molecule on the ground state PES. The dynamics in real and reciprocal space can be seen on the Figure 4.11 a) and b). When we study the effect of finite x-ray pulse lenghts on this scattering measurements we see a different picture from the dissociation case. The Figures 4.12 a) and b) show us that very short probe pulses are necessary for a time resolved measurement of a process confined to a realtively small area on the PES, while pulses of length 


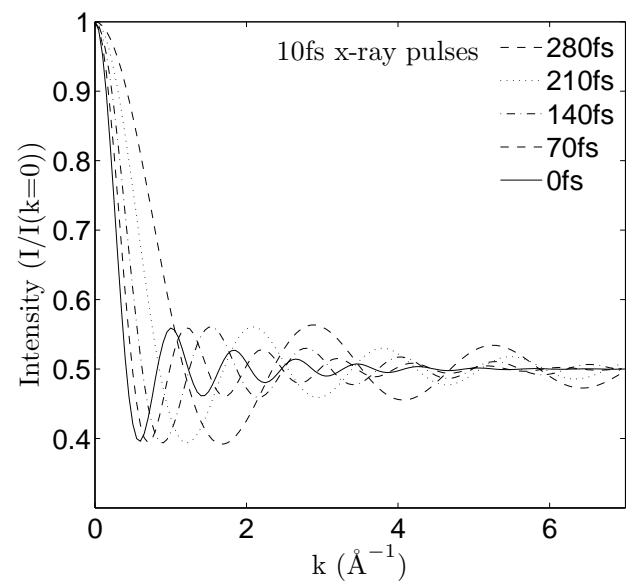

(a)

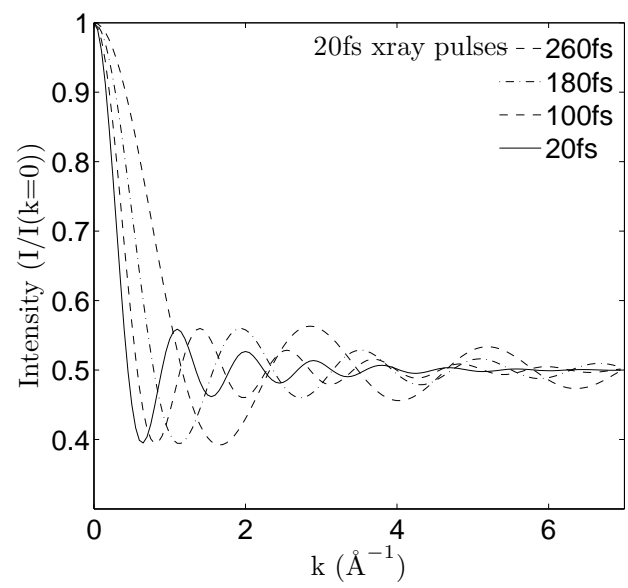

(b)

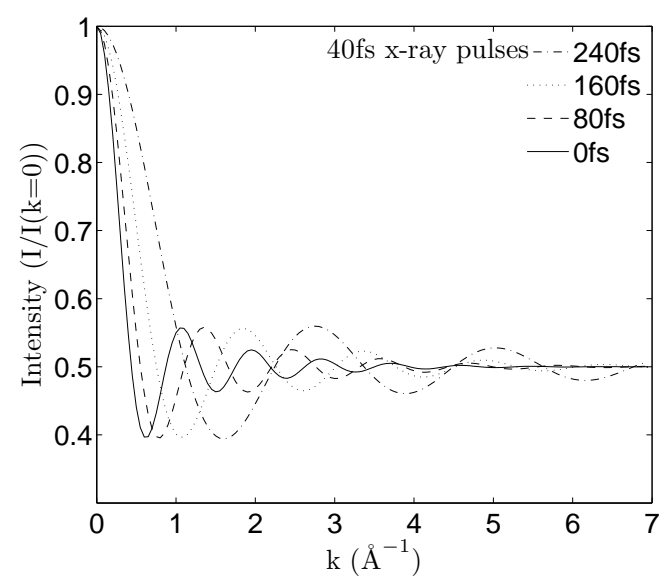

(c)

Figure 4.8. The dissociation dynamics of $\mathrm{I}_{2}$, probed at indicated times after the photoexcitation, with x-ray pulses of finite length: a) 10fs x-ray pulses b) 20fs x-ray pulses c) 40fs x-ray pulses

$\sim 80$ fs can measure only the average thermal behavior. 


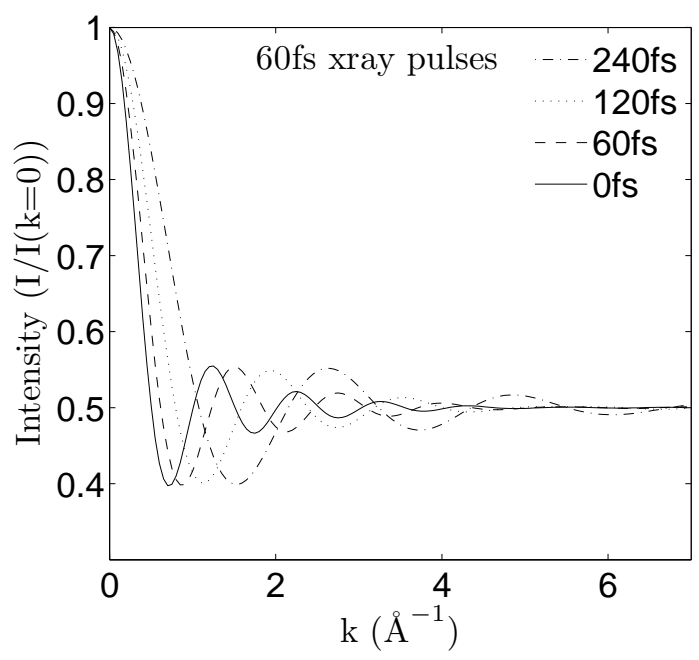

(a)

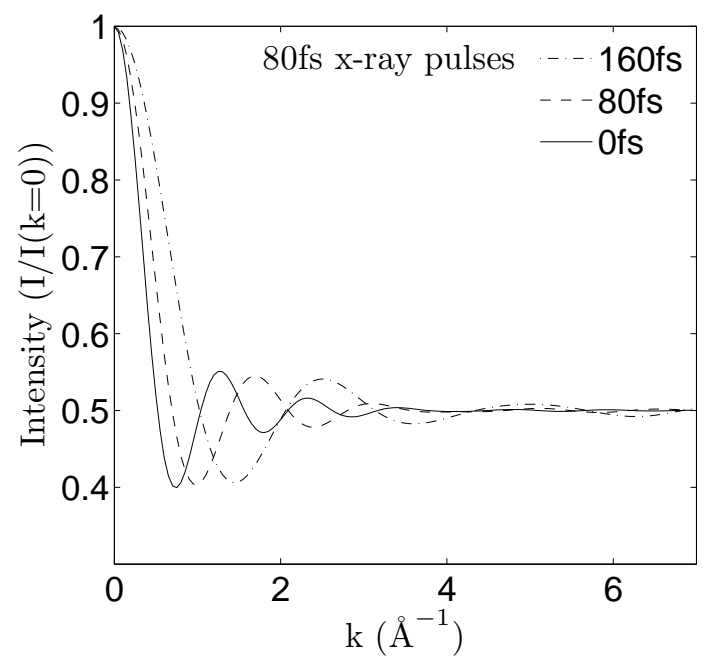

(b)

Figure 4.9. The dissociation dynamics of $I_{2}$ probed with $x$-ray pulses of finite length a) 60fs x-ray pulses b) 80fs x-ray pulses 


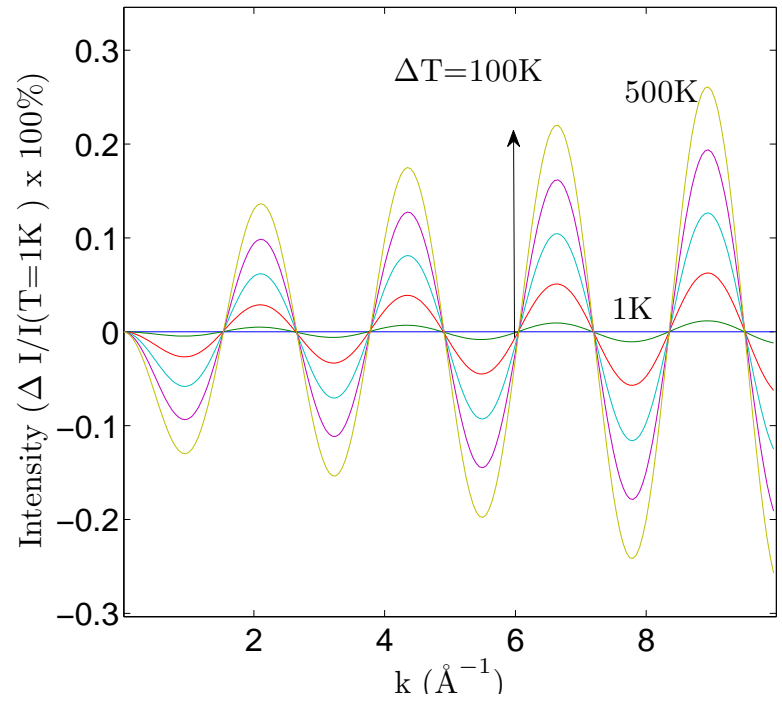

Figure 4.10. Scattering intensity difference in $\Delta I / I(T=1 K)$ for iodine in thermal equilibrium with Boltzmann distribution of vibrational states at temperatures $\mathrm{T}=1 \mathrm{~K}$ to $\mathrm{T}=500 \mathrm{~K}$ 


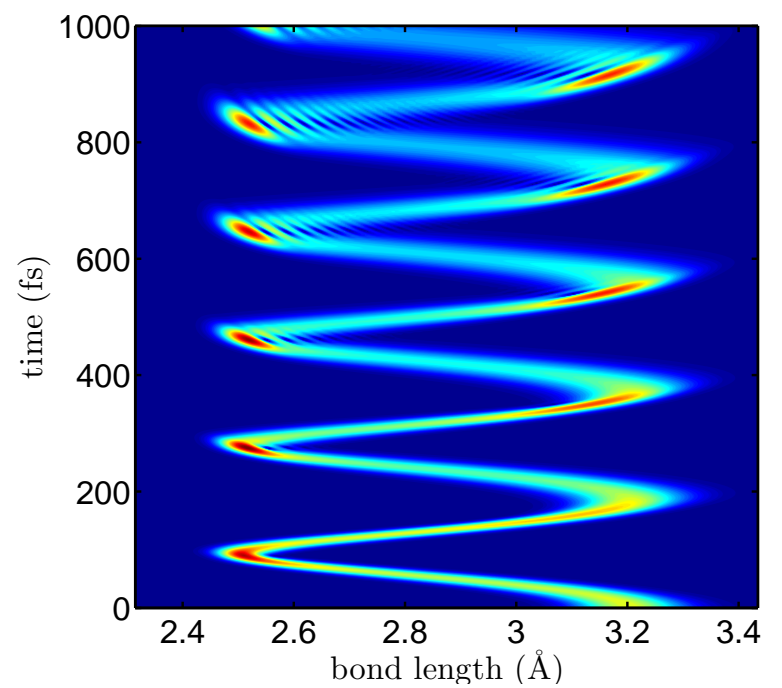

(a)

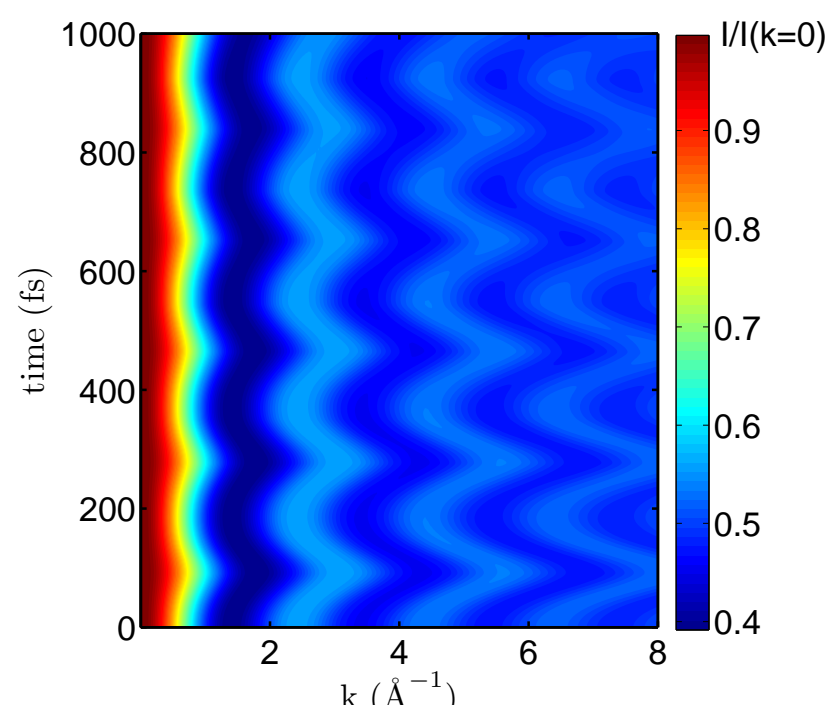

(b)

Figure 4.11. Coherent relaxation of the $I_{2}$ vibrational wave packet on the ground state energy surface a) in real space b) x-ray scattering signal (reciprocal space) 

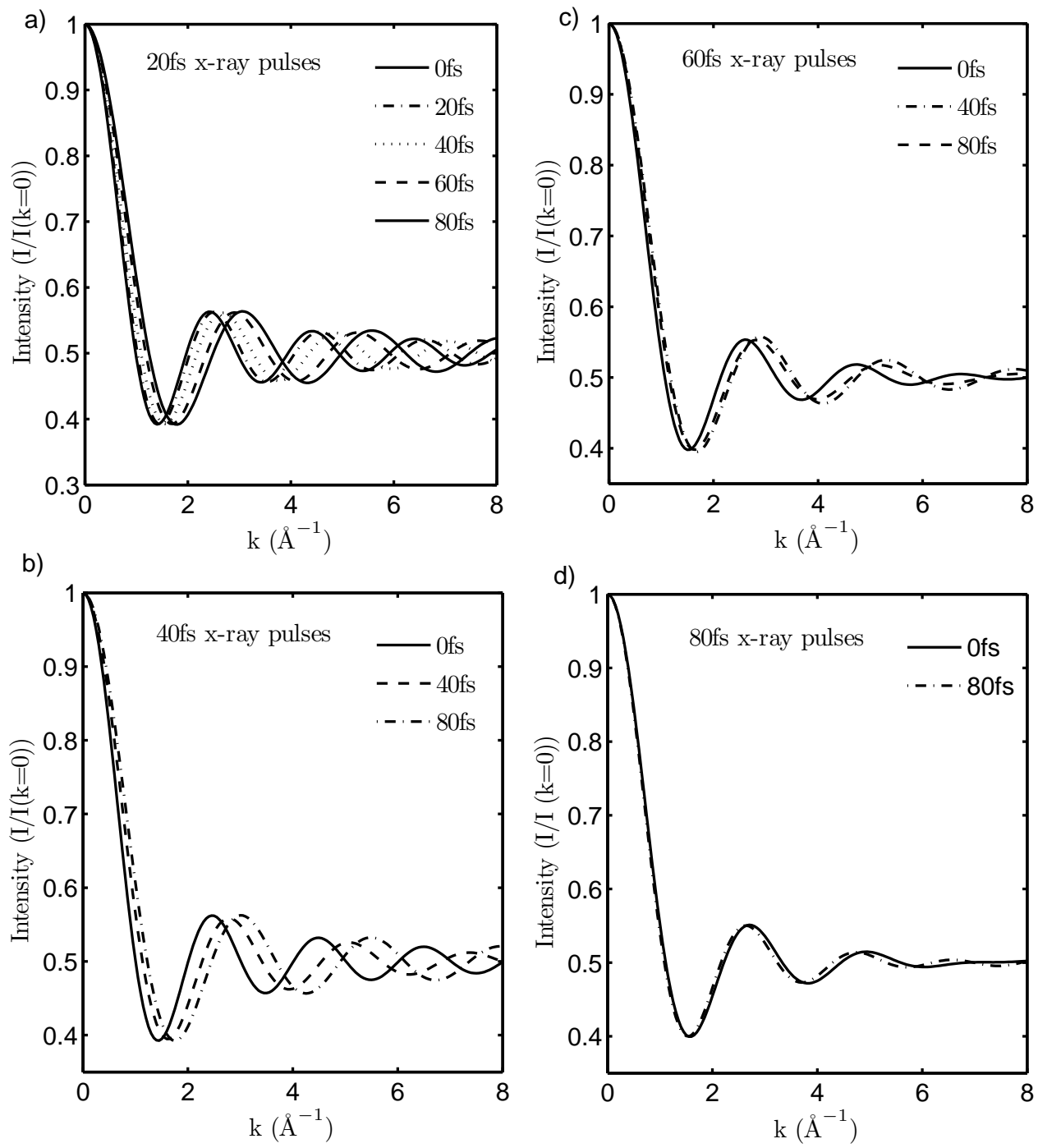

Figure 4.12. Coherent relaxation of $I_{2}$ on the ground state energy surface probed with $\mathrm{x}$-ray pulses of finite length a) $20 \mathrm{fs}$ x-ray pulses b) $40 \mathrm{fs}$ x-ray pulses c) $60 \mathrm{fs}$ x-ray pulses d) 80fs x-ray pulses 


\subsection{Conclusions}

The modeling of the x-ray scattering of molecular iodine brought forward a number of interesting observations. First of all even a very small electron density change, as represented by the HOMO-LUMO excitation gives a non-negligible signal contribution when dealing with single molecule or potentially aligned samples. Due to the symmetry of the $I_{2}$ molecule the small signal change that corresponds to electron density change caused by different orbital occupation could become observable for aligned systems. The change of the electronic state is visible on the scattering spectrum in our simulation with about $1 \%$ of signal change at $\mathrm{k}_{z}, \mathrm{k}_{x}=0 \AA^{-1}$ in the vicinity of $\mathrm{k}_{y}=1 \AA^{-1}$.

The notion of studying coherent molecular processes in a time-resolved x-ray scattering experiment, as for example time resolved wide angle x-ray scattering, is also intriguing. Especially since even the control of wave packet motion by focusing the delocalized wave packet has been proposed ${ }^{52}$ and experimentally proven. 53 The wave packet coherence provides a boost for the scattering signal since it provides new equivalent structure for the constructive interference. The typical time duration of coherent processes on the order of tens to hundreds of femtoseconds, depending on the environment (gas or liquid sample), presents a limitation for the possible probe pulse duration. At the same time the pulse duration is limited in the same way by the necessity for high resolution in the time domain in the time-resolved experiments.

The choice of probe pulse length also depends on the sort of the studied process, which can be either periodic or aperiodic in time. Naturally, in the case of periodic processes, such as relaxation of the wave packet on an energy surface after an excitation event, the probe pulse length has to be just a fraction of the period of the process. In our test case on the relaxation of iodine on the ground state surface that has a period of about 80 fs the probe pulse must be shorter than about $60 \mathrm{fs}$. The more important is the case of aperiodic processes, as is for example the iodine dissociation. Here the pulse length can be longer. In our case of the first stage of the dissociation, or lengthening of the bond, an event that was found to take about 300 fs can be measured by probe pulses of up to about 80 to even $100 \mathrm{fs}$. 


\section{Chapter 5}

\section{X-ray Scattering on Aligned Molecules}

\subsection{Introduction}

With the build up of the new x-ray sources the interest in their utilization for detailed measurements of structure and dynamics of various molecular systems mainly bio-molecules and their dynamics — has naturally increased.

Already in the work [5] Neutze et al consider the implications that the combination of high intensity and short pulse lengths of the FEL has for structural studies. The authors there evaluate the feasibility of structural measurements of samples consisting of a single molecule, while taking into account that the Coulomb explosion takes place due to the high intensity of the laser pulse. Although this proposed technique avoids the problems with crystallization, which has been a necessity in structural studies using conventional x-ray sources, it also introduces new challenges for the experimentalists. Some of the theoretical implications of the $\mathrm{x}$-ray scattering measurements using very strong probe pulses are presented in the next Chapter 6. Generally one would prefer to study molecular systems and their chemical dynamics in their natural environments, e.g. in solutions. In order to introduce a periodicity into such a system various methods of alignment have been proposed and in experimentally performed for small test systems.

Originally the interest in manipulating the orientation of molecules in space 
comes from the studies of chemical reactions which are in most cases dependent on the mutual orientation of the reactants. The most promising method emerging is the alignment under the influence of laser fields. A number of theoretical and experimental works has been published in recent years on the laser induced alignment of molecules. $\frac{1021103 / 104105}{} \mathrm{~A}$ review of alignment techniques with the main focus on alignment in strong laser fields can be found in [106]. Here we offer a summary of different alignment techniques and the conditions and problems constraining the efficiency of alignment in experiments.

The alignment of molecules by a laser field is based on the interaction of a strong non-resonant laser field with an induced dipole moment of a molecule. This interaction creates an energy minimum for the alignment along the polarization axis of the field and so limits the free rotation of the molecules. Under these conditions molecules librate in a limited angular range.

Depending on the pulse length of the aligning laser one distinguishes between two main alignment set-ups. In the first case the laser pulse, in the following represented by the laser pulse length $\tau$, is much longer than the rotational period of the molecule $\tau \gg \tau_{\text {rot }}$ and we can talk about a continuous wave alignment laser pulse. This so called adiabatic alignment, vanishes after the alignment pulse is turned off. The second case, when $\tau \ll \tau_{\text {rot }}$ is the so called non-adiabatic alignment. Here the alignment predictably periodically returns after the laser pulse is turned off and so we can talk about field free alignment. Under specific $\tau$ conditions the alignment strengthens with the laser off.

The laser alignment technique is fast emerging method of choice in experiments. It is assumed that the vibrational degrees of freedom can be neglected and the alignment is said to be dependent only on the rotational degree of freedom of the molecule. In order to minimize rotations, embedding of molecules in rare gas liquids, such as argon, has been proposed.

For x-ray scattering the degree of alignment is an important property since it introduces additional "periodicity" to an otherwise disorganized system and thus enhances the characteristic details in the scattering signal. As we will show, even the most basic one dimensional (1D) alignment offers additional structural information in comparison to a completely amorphous sample. We talk about 1D alignment when the rotation about one of the axis of the molecule is hindered. This simply means that the molecule can freely rotate around one axis, without 
orientation restrictions about the remaining axis. It provides a good compromise between added demands on the experimental set-up and enhanced sample periodicity, and so enhancing constructive interference in the scattering signal. The x-ray scattering spectra of tested molecules with the one dimensional alignment provide the extra structural information necessary to study even relatively small structural changes and so enable for example the time-resolved studies of systems undergoing structural change. Even higher control over the periodicity in the sample provides the tree dimensional alignment (3D) and orientation control. This would to some extend simulate single-molecule scattering, however orienting molecules is a much more difficult problem than simple alignment.

In recent years a number of works concerned with various methods of x-ray imaging applied on aligned systems has been published, dealing with small test systems such as $\mathrm{Br}_{2}{ }^{101}$ etc. In this Chapter we will study whether sample alignment is a suitable option for x-ray scattering studies of systems with sizes ranging from tens of atoms to hundreds of atoms as in various organic molecules and bio-molecular systems, since these are traditionally in the center of interest for structure and dynamics studies.

As an additional motivation for the interest in the aligned samples we should add that while already the short pulse length of the FEL, on the level of femtoseconds, promises many interesting applications for measurements, methods for achieving even shorter pulse lengths, on the level of atto-seconds, has been proposed. $107 / 108$ A side effect of this is, however, a decrease in the pulse intensity. This intensity would not be sufficient for one molecule measurements. On the other hand it gives us an incentive to look into the feasibility of time-resolved measurements of aligned samples.

\subsection{X-ray Scattering on Aligned Systems}

Since this Chapter is mainly concerned with the alignment effects on the scattering spectra, the calculated scattering intensity will be treated using the classical approach (Debye approximation) with no delocalized electron densities.

In the case of the so called one-dimensional alignment — when the molecule can freely rotate along one axis - we deal with a straightforward integration in 


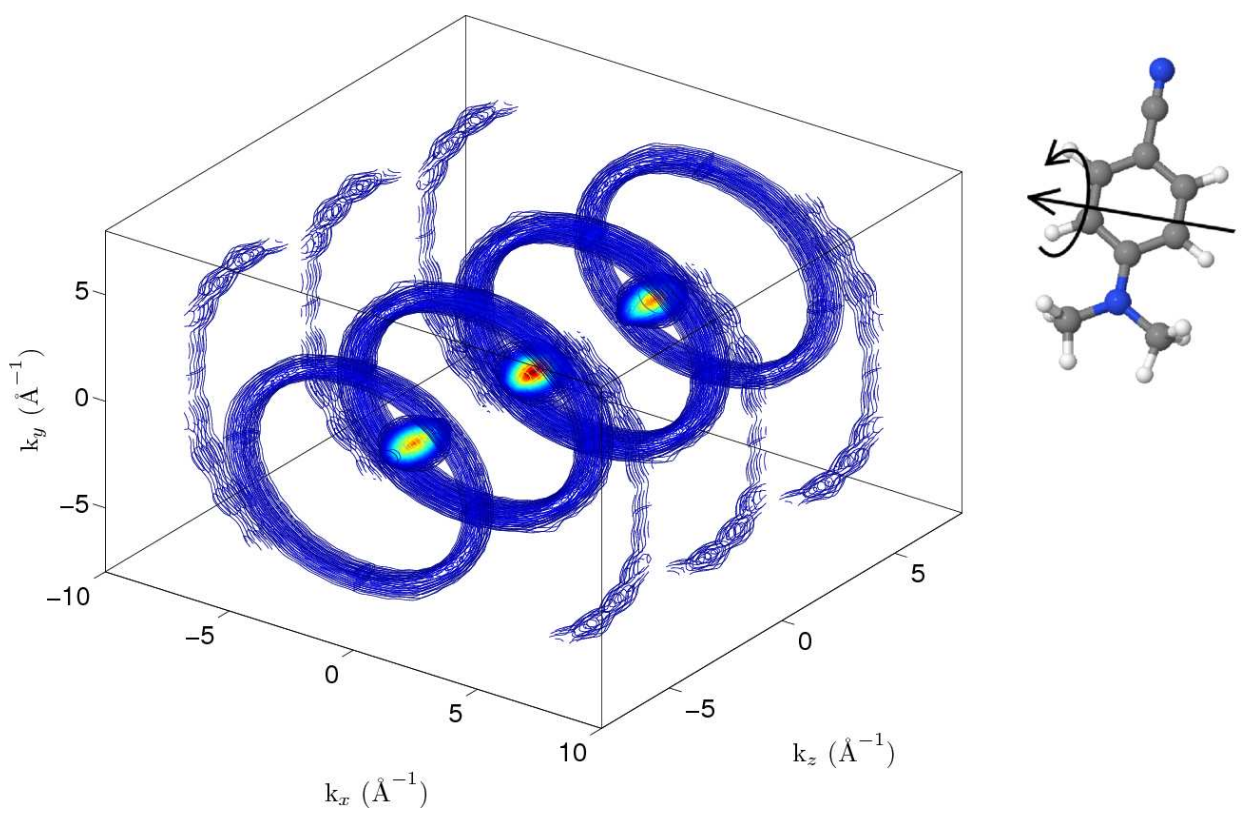

Figure 5.1. Scattering map of an ensemble of 32 DMABN molecules aligned in onedimension

one rotational degree of freedom

$$
I(k, \theta)=\frac{1}{2 \pi} \int_{0}^{2 \pi} d \phi\left|\mathcal{F}_{k, \phi, \theta}\{\rho(\mathbf{r})\}\right|^{2}
$$

where one can conveniently include the uncertainty in confinement in $\theta$ with the probability distribution of the uncertainty $p(\theta)$ as:

$$
I(k, \theta)=\frac{1}{2 \pi} \int_{0}^{\theta_{\max }} p(\theta) \sin \theta d \theta \int_{0}^{2 \pi} d \phi\left|\mathcal{F}_{k, \phi, \theta}\{\rho(\mathbf{r})\}\right|^{2}
$$

where $\theta_{\max }$ is defined as the angle where $p\left(\theta_{\max }\right) \sim 0$ and $\int_{0}^{\theta_{\max }} p(\theta) \sin \theta d \theta=1$. An example of scattering intensity of one-dimensionally aligned molecules of 4,4'dimethylaminobenzonitrile (DMABN) can be seen in Figure 5.1

As the calculated scattering intensity maps in this Chapter show the one dimensional alignment offers a significant improvement on the information about the geometry of the studied sample, when compared to the sample with random 
orientations. The uncertainty in the alignment angle $\theta$ is an important parameter. Due to convenience in the angular integration we chose to approximate the angular uncertainty distribution by a $(\cos x)^{2 \alpha}$ function, which for large $\alpha$ very well approximates the Gaussian function $\exp \left(-\alpha x^{2}\right)$ with the full width at half maximum related to the parameter $\alpha$ as $\sqrt{\log 2 / \alpha}$.

The most notable property of the 1D alignment is the dependence of the spectra on the alignment axis. The Figures 5.2 and 5.3 show the $1 \mathrm{D}$ alignment scattering intensities of stilbene with respect to different alignment axis. The difference in the scattering spectra is significant. This is a promising feature since the main polarizability axis, which coincides with the laser alignment axis, can be modified by attaching various polarizable groups to the molecule.

Keeping the alignment axis constant we can show the importance of the precision in the alignment. The Figures 5.4 and 5.5 show the same alignment geometry as the Figure 5.3 but with an uncertainty in alignment axis of $10^{\circ}$ and $30^{\circ}$ respectively. The strong scattering features of the well aligned molecules fast diminish with growing uncertainty in the alignment angle. However, even the uncertainty distribution with the FWHM of $30^{\circ}$ still offers a scattering spectra that is much improved when compared to a fully spherically symmetric scattering spectra of a sample with randomly oriented molecules. The spectra of randomly oriented sample are depicted in Figure 5.15 b) as a part of next Section of this Chapter. In the experiments with the 1D laser alignment reported in [109] the smallest values of $\Delta \theta$ achieved is in the range of $20^{\circ}$. 


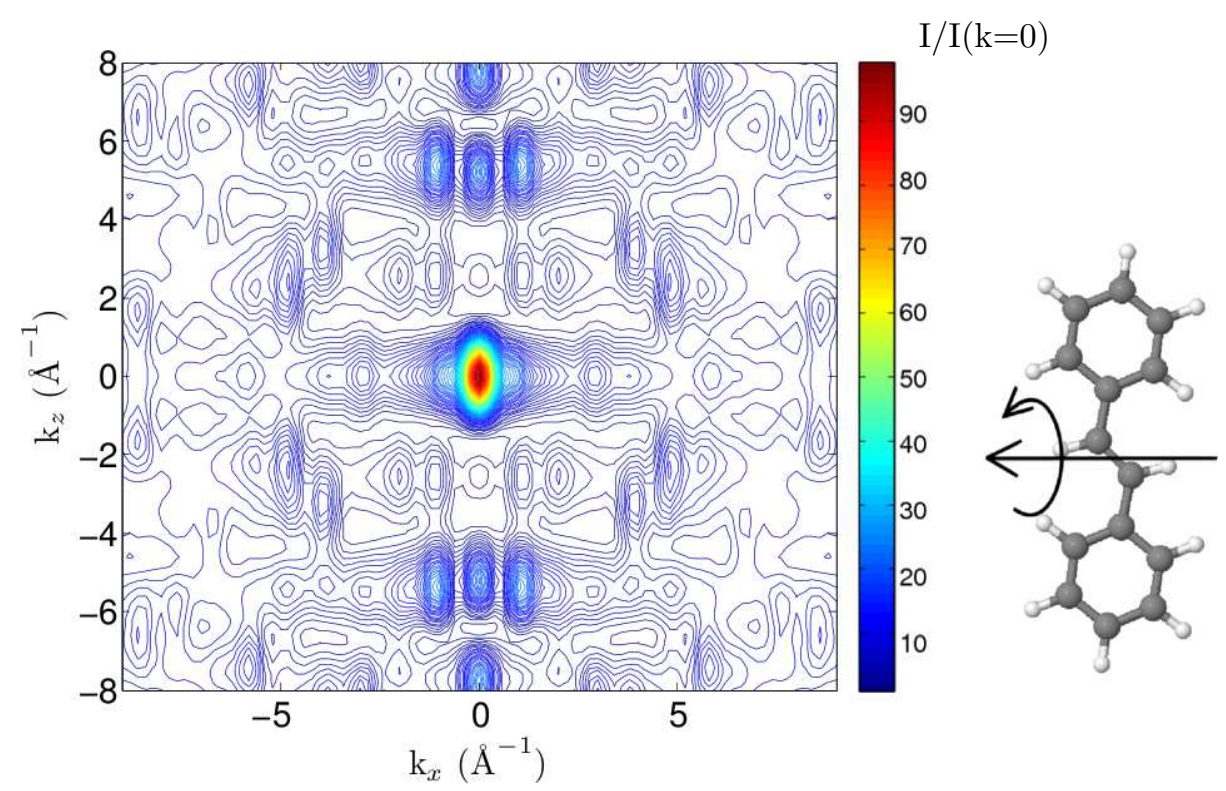

(a)

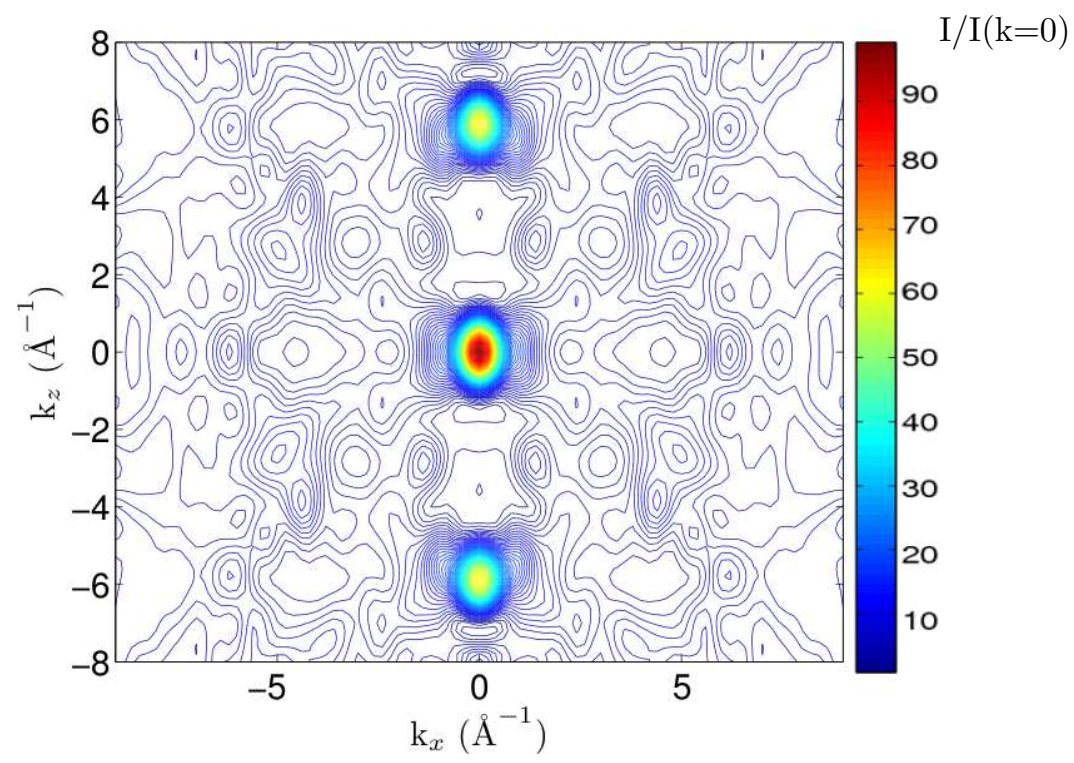

(b)

Figure 5.2. One-dimensional alignment of stilbene with the alignment axis perpendicular to the main moment of inertia axis of stilbene a) trans with $0^{\circ}$ uncertainty in alignment b) cis with $0^{\circ}$ uncertainty in alignment 


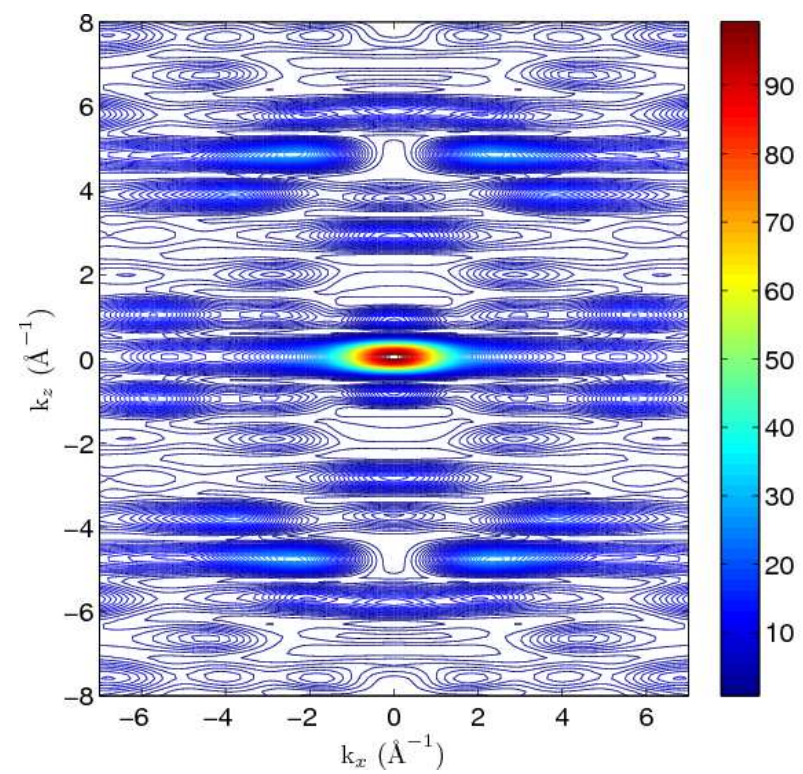

$\mathrm{I} / \mathrm{I}(\mathrm{k}=0)$

90

80

70

60

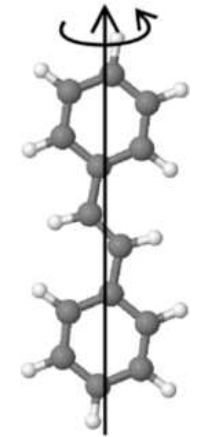

(a)

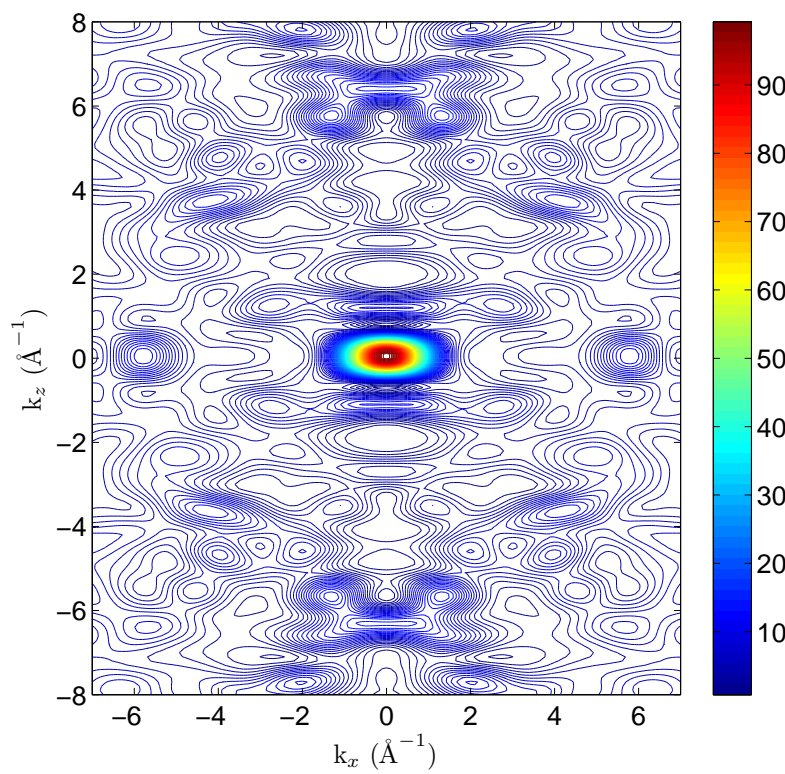

$\mathrm{I} / \mathrm{I}(\mathrm{k}=0)$

80

70

60

50

40

30

20

10

(b)

Figure 5.3. One-dimensional alignment of stilbene with the alignment axis equal to the moment of inertia axis of stilbene a) trans with $2^{\circ}$ uncertainty in alignment b) cis with $2^{\circ}$ uncertainty in alignment. See text for details 


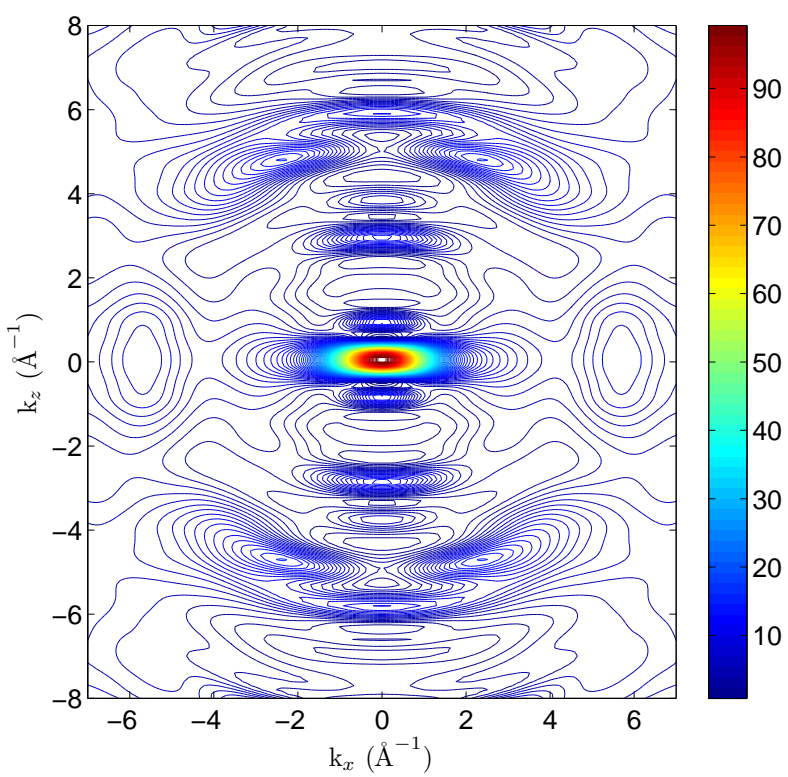

(a)

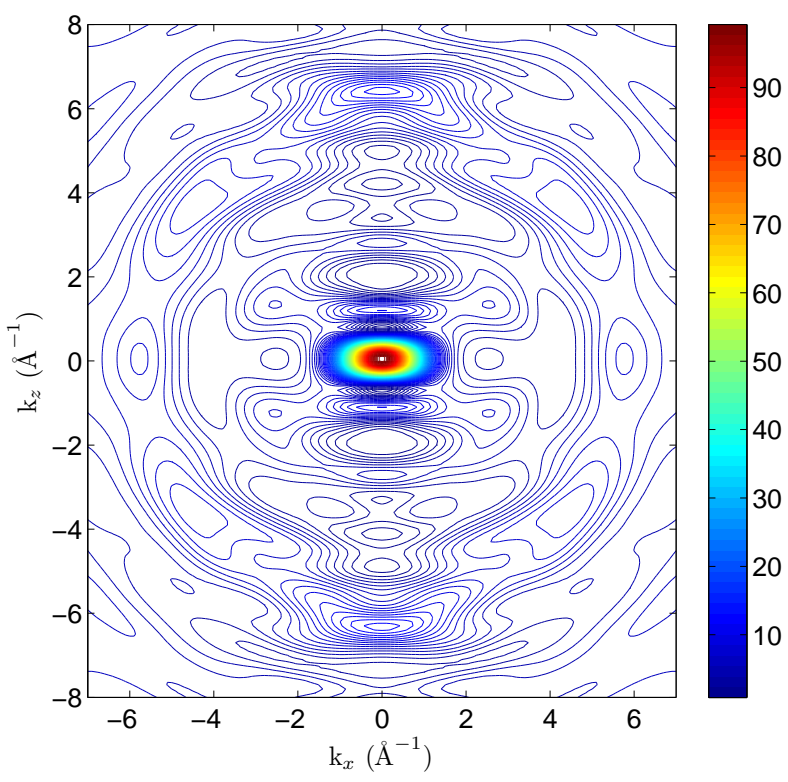

(b)

Figure 5.4. Stilbene scattering from a) trans with $10^{\circ}$ uncertainty in alignment b) cis with $10^{\circ}$ uncertainty in alignment 


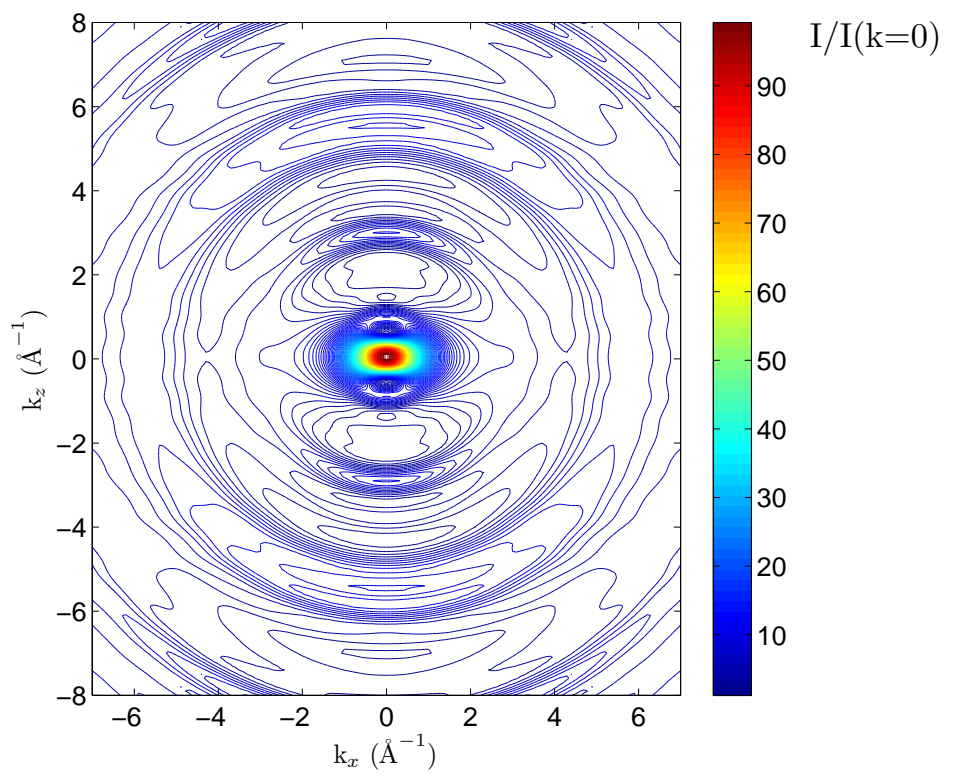

(a)

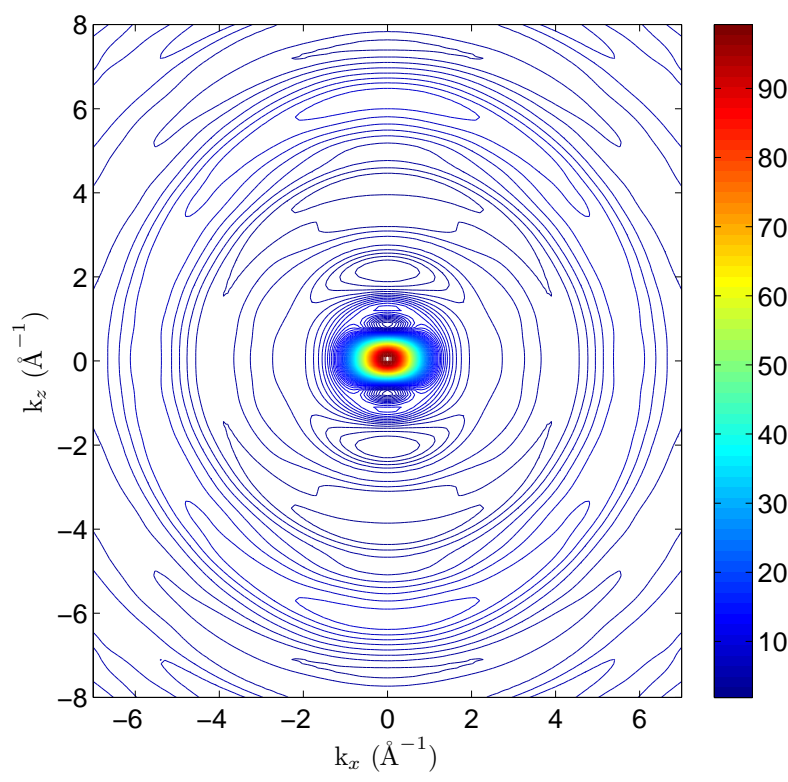

(b)

Figure 5.5. Scattering from a) trans and b) cis stilbene geometries with $30^{\circ}$ uncertainty in alignment 

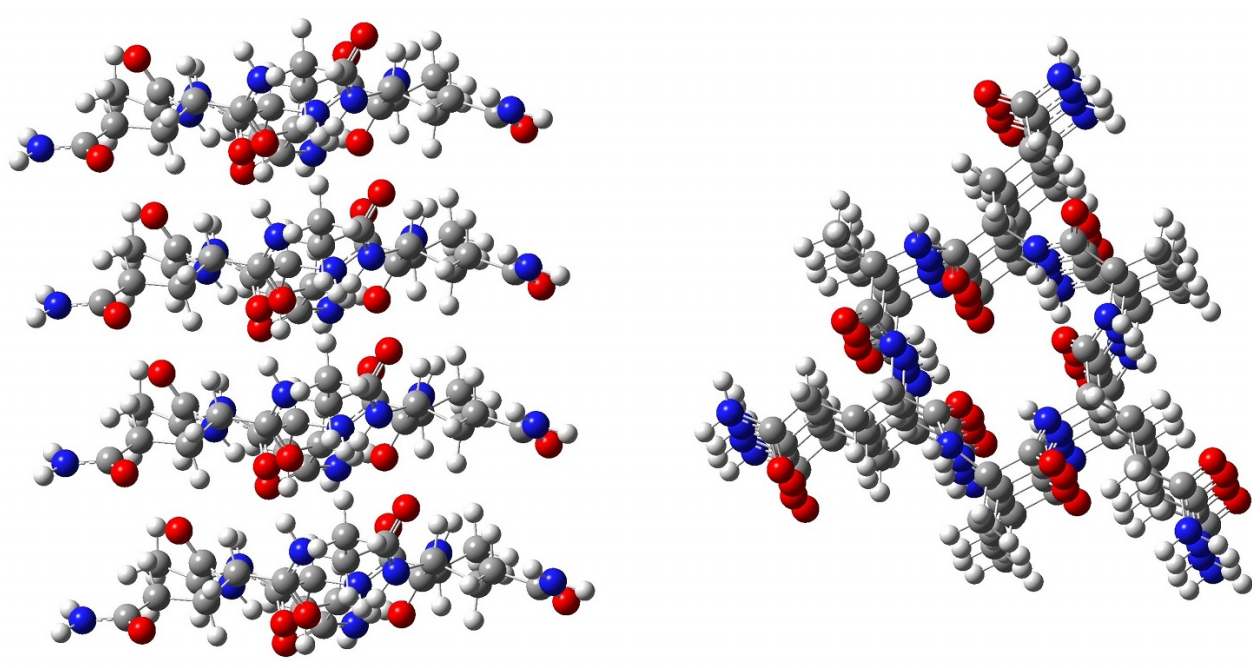

Figure 5.6. Structure of the peptide nanotube

The scattering spectra of larger molecules are much more rich in features, with a much stronger intensity decrease with growing scattering vector $k$. We compare the effect of alignment and the uncertainty of alignment for larger molecules using the example of peptide nanotubes (structure shown in Figure 5.6). The scattering intensity of $1 \mathrm{D}$ aligned ensemble of these peptide nanotubes is shown in Figure 5.7. Even though the scattering signal has been averaged over rotations around one particular axis to represent the 1D alignment this intensity map is still relatively rich in detail. We focus to a smaller $k$-range in Figure 5.8, which is a more experimentally realistic picture due to the strong intensity decrease with larger $k$. Comparing the effect of uncertainty in alignment for an ensemble of larger molecules, even the intensity spectra with the uncertainty in alignment of $20^{\circ}$ (Fig 5.8 b) remains relatively rich and so even the simple 1D alignment is potentially beneficial for improved x-ray scattering measurements when performed on larger molecules.

Theoretically the three dimensional alignment offers the best structural resolution of the studied sample. The expression for the intensity distribution including the uncertainty in the alignment $\Delta \theta$ and $\Delta \phi$ and the probability distributions of 


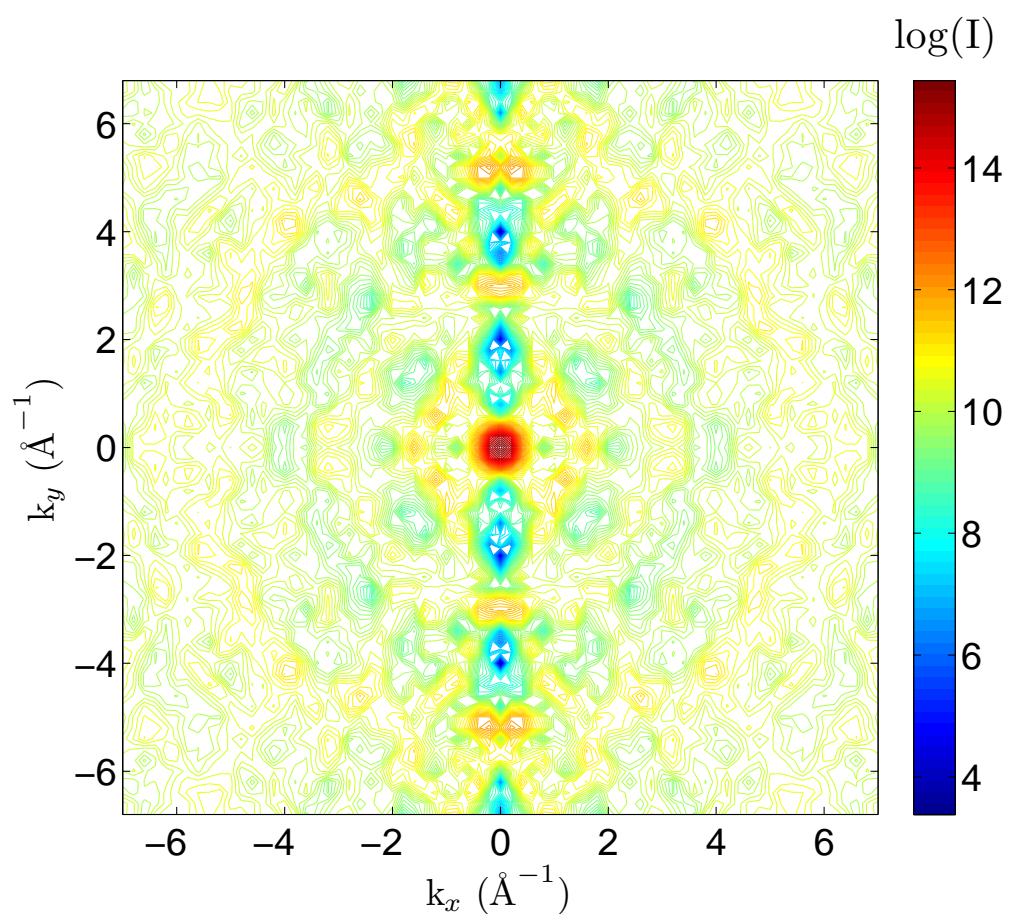

Figure 5.7. 1D alignment of peptide nanotubes with intensity in logarithmic scale

the uncertainty $p_{1}(\theta), p_{2}(\phi)$ is simply:

$$
I(k, \theta)=\int_{0}^{\theta_{\max }} p_{1}(\theta) \sin \theta d \theta \int_{0}^{\phi_{\max }} p_{2}(\phi) d \phi\left|\mathcal{F}_{\mathbf{k}}\{\rho(\mathbf{r})\}\right|^{2}
$$

with $p_{1}\left(\theta_{\max }\right) \sim 0$ and $p_{2}\left(\phi_{\max }\right) \sim 0$. Illustrative examples of scattering intensity with three dimensional alignment coincide with the single molecule scattering examples and can be found in the previous Sections on x-ray scattering from stilbene and iodine. 


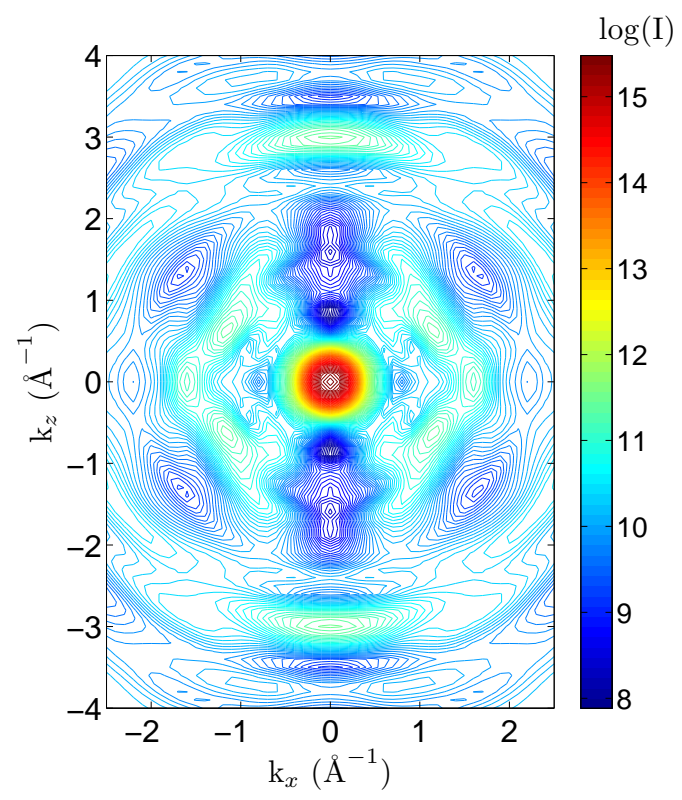

(a)

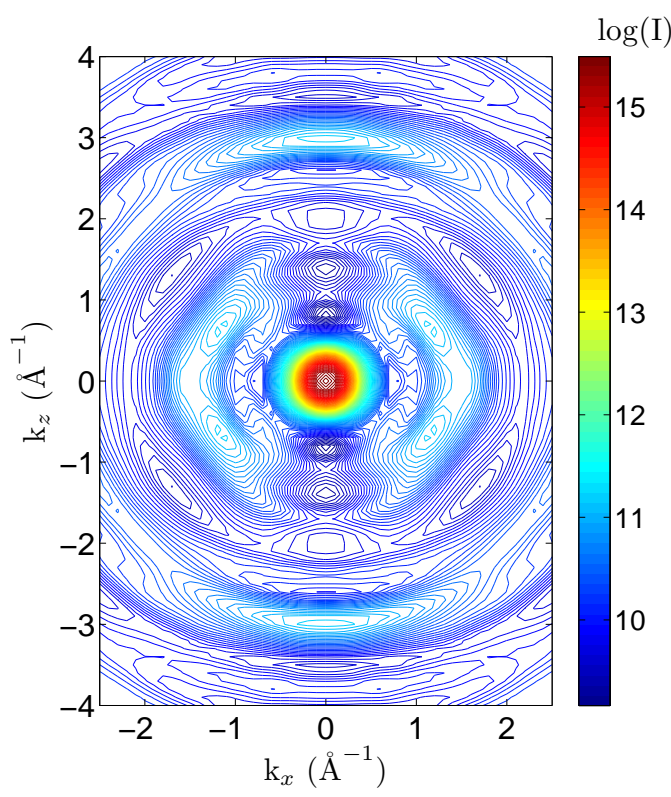

(b)

Figure 5.8. 1D alignment of peptide nanotubes; a) uncertainty in alignment $0^{\circ} \mathrm{b}$ ) uncertainty in alignment $20^{\circ}$; Intensity in logarithmic scale 


\subsection{Time-Resolved Scattering on Aligned Systems}

Scattering on aligned samples gives the information about the electron density projection on the alignment axis. This offers a number or interesting opportunities for measurements. If one could change the polarizability axis of the molecule one could observe different projections of the density and so to some extent focus on the area of interest - maximum density change corresponding to observed process.

As the previous Section showed, the one dimensional alignment provides additional structural information about the imaged object when compared to randomly oriented sample. In this Section we evaluate if this information is sufficient in order to follow time-dependent processes. We will focus on 1D alignment which poses the lowest experimental demands.

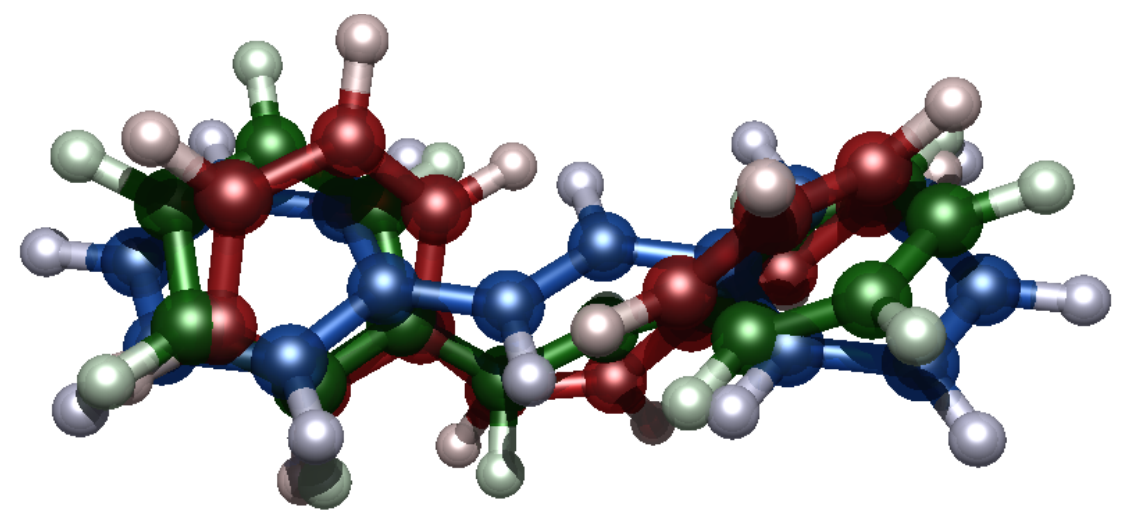

Figure 5.9. Geometry change of aligned stilbene during the photo-isomerisation adjusted to angular momentum change $\delta L=0$, with blue model corresponding to trans stilbene, green to a half-way conformation with dihedral angle of $93.5^{\circ}$ and red to cis conformation

We return to the photoisomerization of stilbene as the test process. The geometry change excluding rotation is depicted in Figure 5.9. The photoisomerization process starts in the trans geometry when the molecular ensemble is aligned with respect to the main moment of inertia axis. Figures 5.10 to 5.14 show the scattering of an aligned system undergoing the photo-isomerisation where the alignment has a $20^{\circ}$ uncertainty. The alignment axis remains the main moment of inertia axis at the given time point. Since we want to examine the sensitivity of the 1D scattering spectra to the geometry change we chose 10 equidistant steps in geometry change on the simplified minimum energy path (see Fig. 3.1), which correspondents to 
about $20^{\circ}$ change of the dihedral angle. The details of the photoisomerization process were described in Chapter 3 .

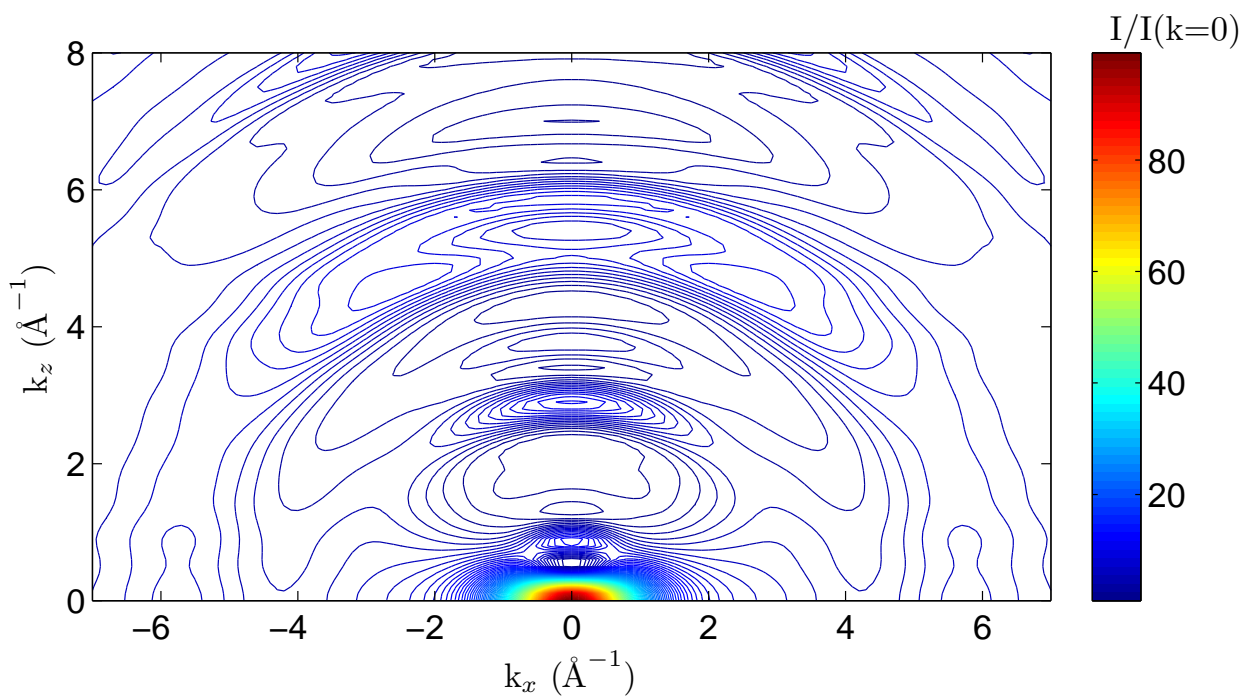

(a)

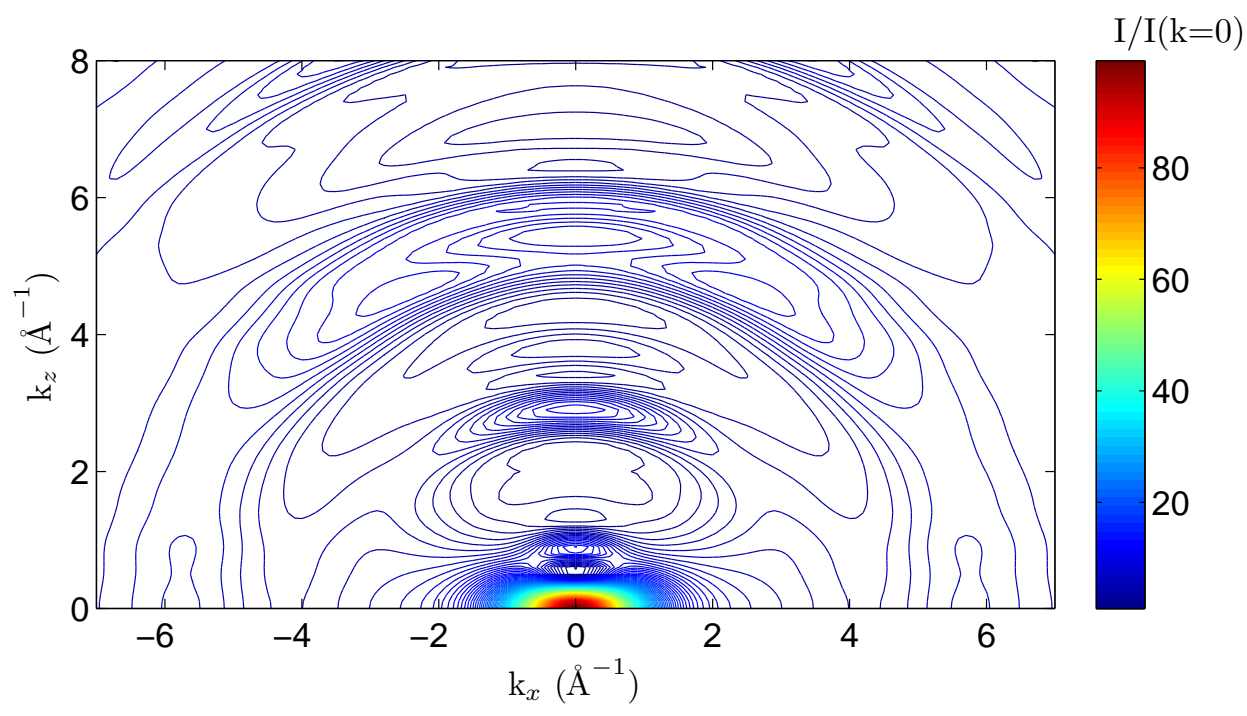

(b)

Figure 5.10. Isomerisation of stilbene; $1 \mathrm{D}$ scattering intensity of the geometry a) trans, b) dihedral angle of $161^{\circ}$ both with an uncertainty in alignment $20^{\circ}$; Color scale in $\%$ of the scattering intensity at $k=0$

The time-resolved 1D spectra are rich enough to enable simplified comparison 


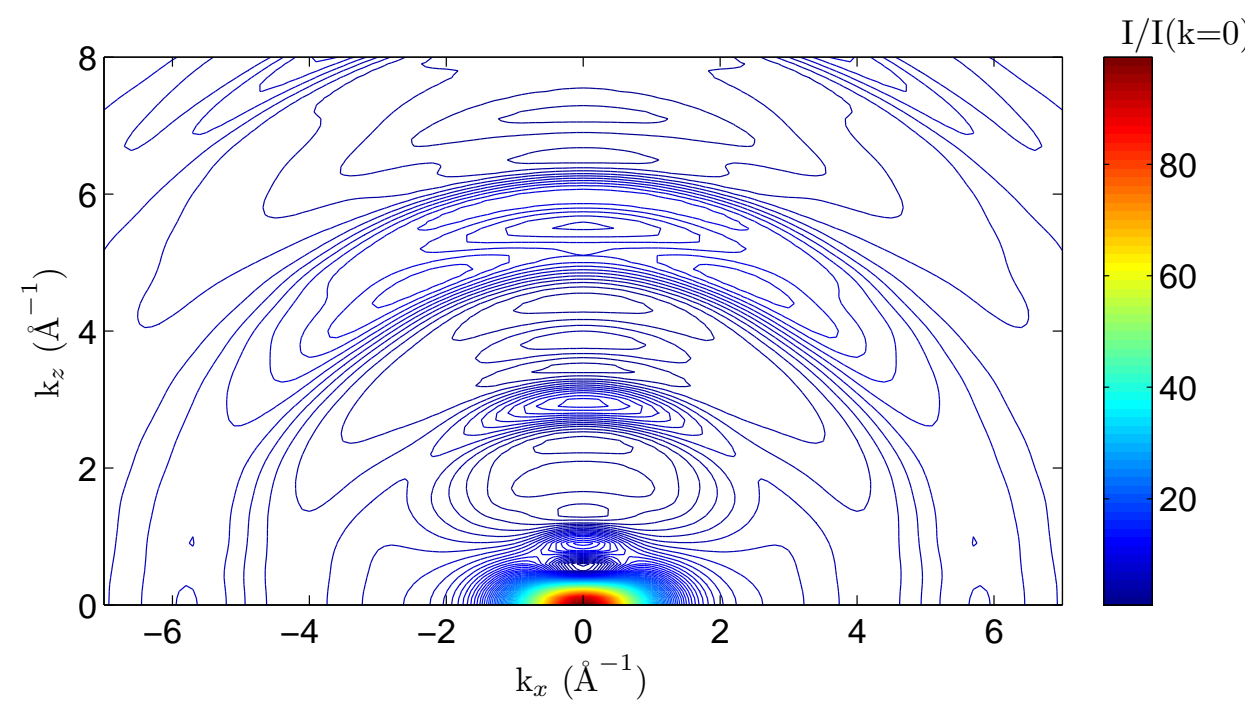

(a)

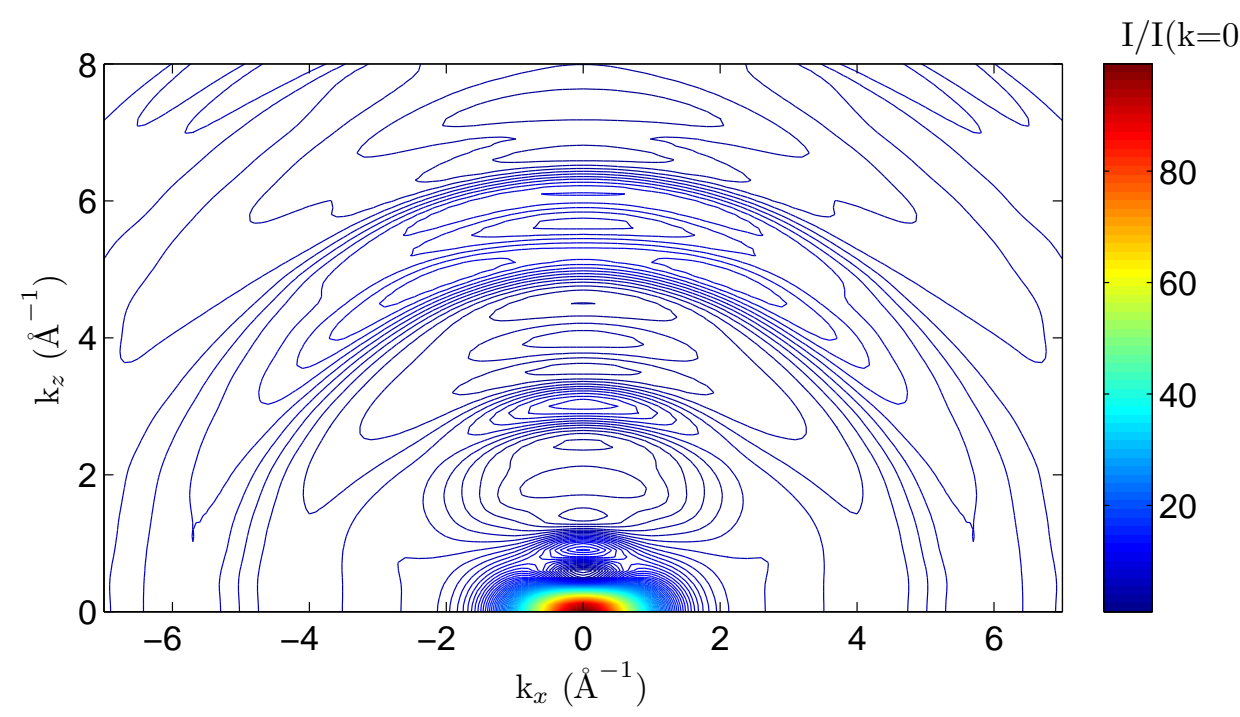

(b)

Figure 5.11. Isomerisation of stilbene; 1D scattering intensity of the geometry with the dihedral angle of a) $142^{\circ}$, b) $122^{\circ}$ with an uncertainty in alignment $20^{\circ}$; Color scale in $\%$ of the scattering intensity at $k=0$

to the spectra from the amorphous sample. Figures 5.10 to 5.14 show a relatively strong signal change even when we focus just on the one-dimensional $I\left(k_{z}, k_{x}=0\right)$. The resulting 1D aligned time-resolved spectra together with the amorphous coun- 


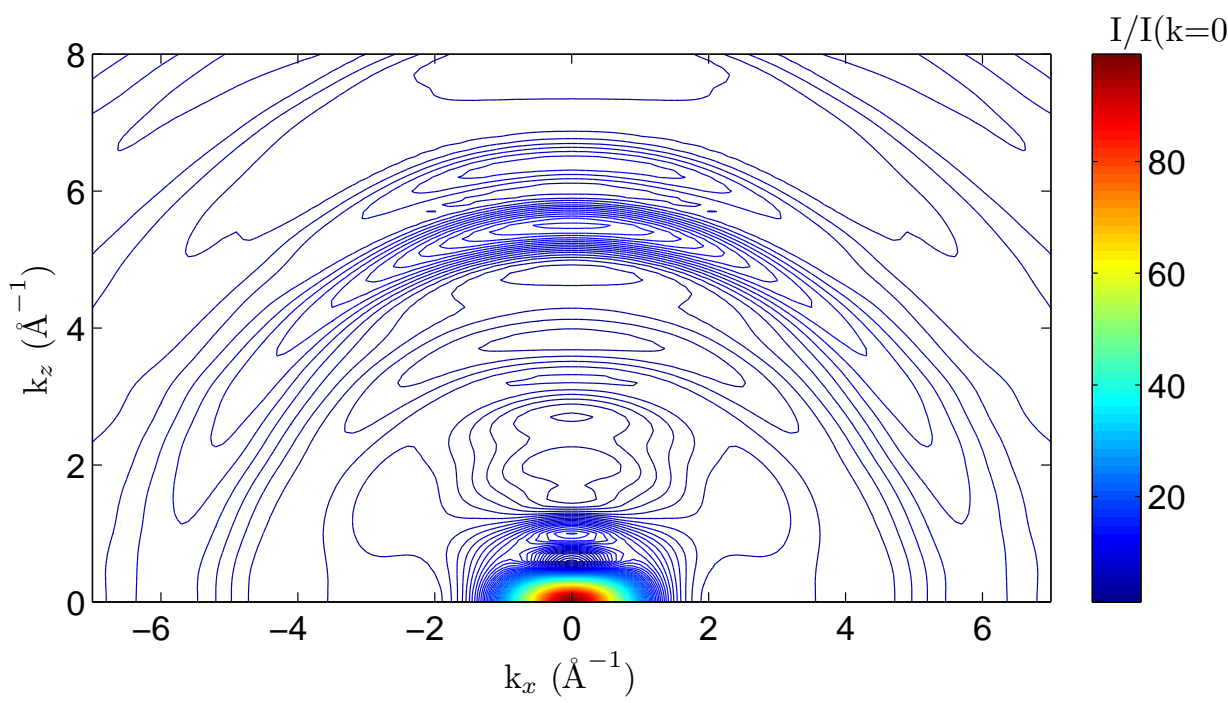

(a)

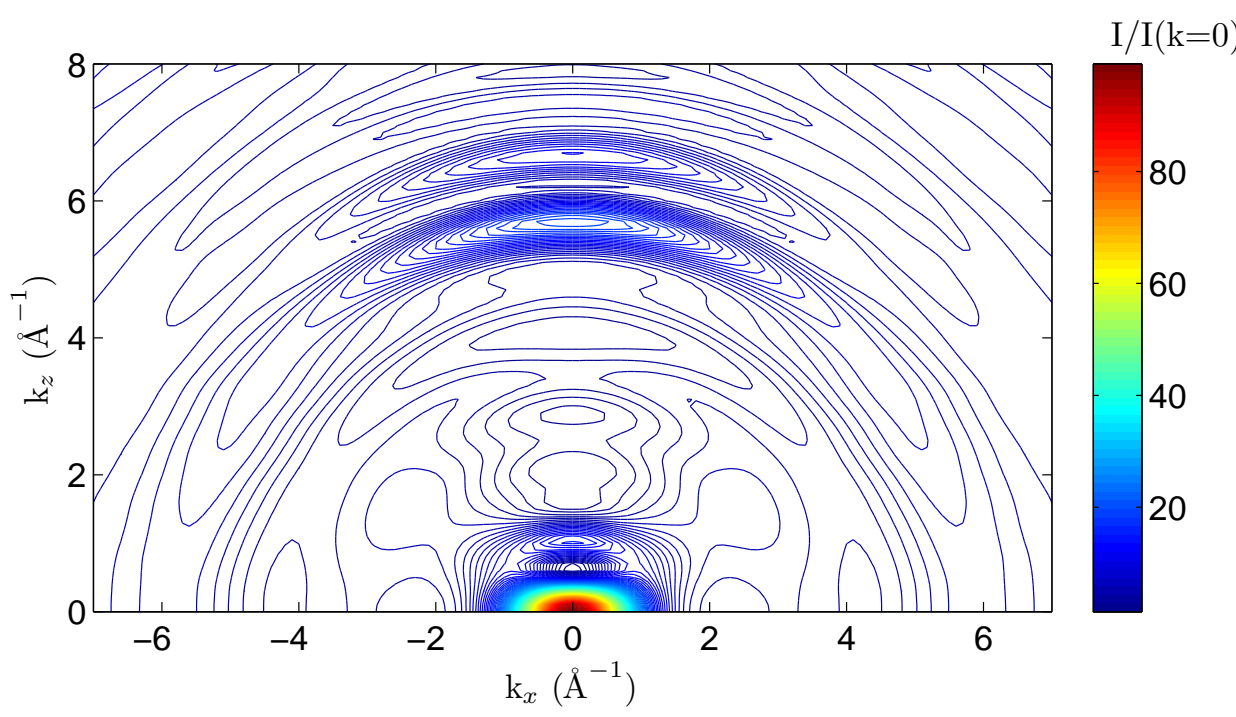

(b)

Figure 5.12. Isomerisation of stilbene; 1D scattering intensity of the geometry with the dihedral angle of a) $103^{\circ}$, b) $84^{\circ}$ with an uncertainty in alignment $20^{\circ}$; Color scale in $\%$ of the scattering intensity at $k=0$

terparts are depicted on the Figures 5.15 showing the comparison of the total intensities, 5.16 showing the difference intensities between the trans geometry and the geometry with the given dihedral angle, and 5.17] shows the intensity differ- 


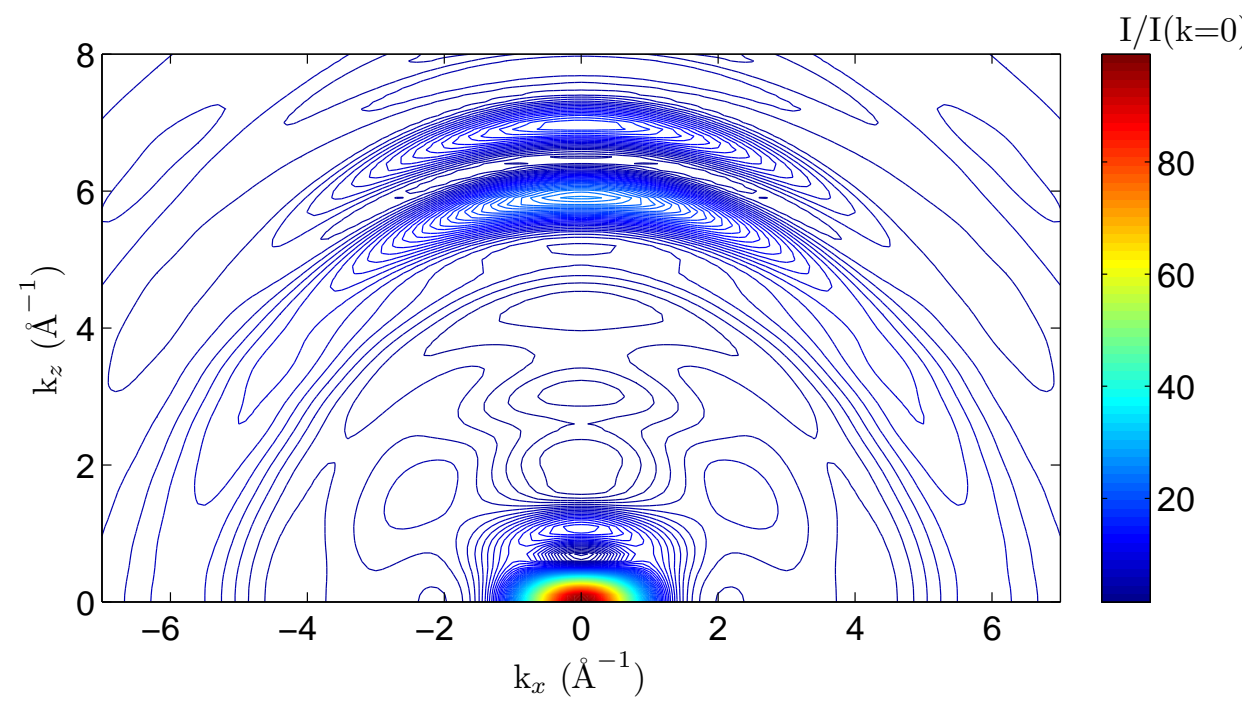

(a)

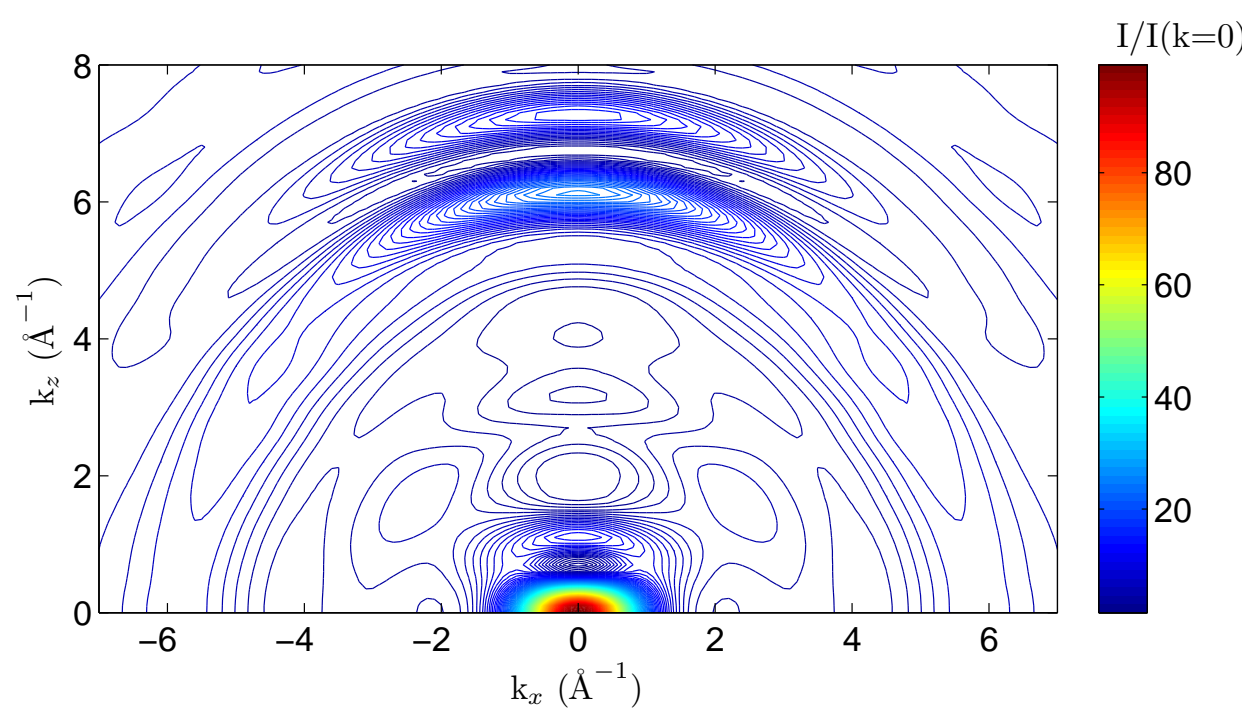

(b)

Figure 5.13. Isomerisation of stilbene; $1 \mathrm{D}$ scattering intensity of the geometry with the dihedral angle of a) $65^{\circ}$ b) $45^{\circ}$ with an uncertainty in alignment $20^{\circ}$; Color scale in $\%$ of the scattering intensity at $k=0$

ence between two consecutive geometries with the dihedral angle difference of $10^{\circ}$. As we can see the even the reduced $I\left(k_{z}, k_{x}=0\right)$ intensity $1 \mathrm{D}$ scattering maps enable much better resolution for structural changes than the spectra from the 


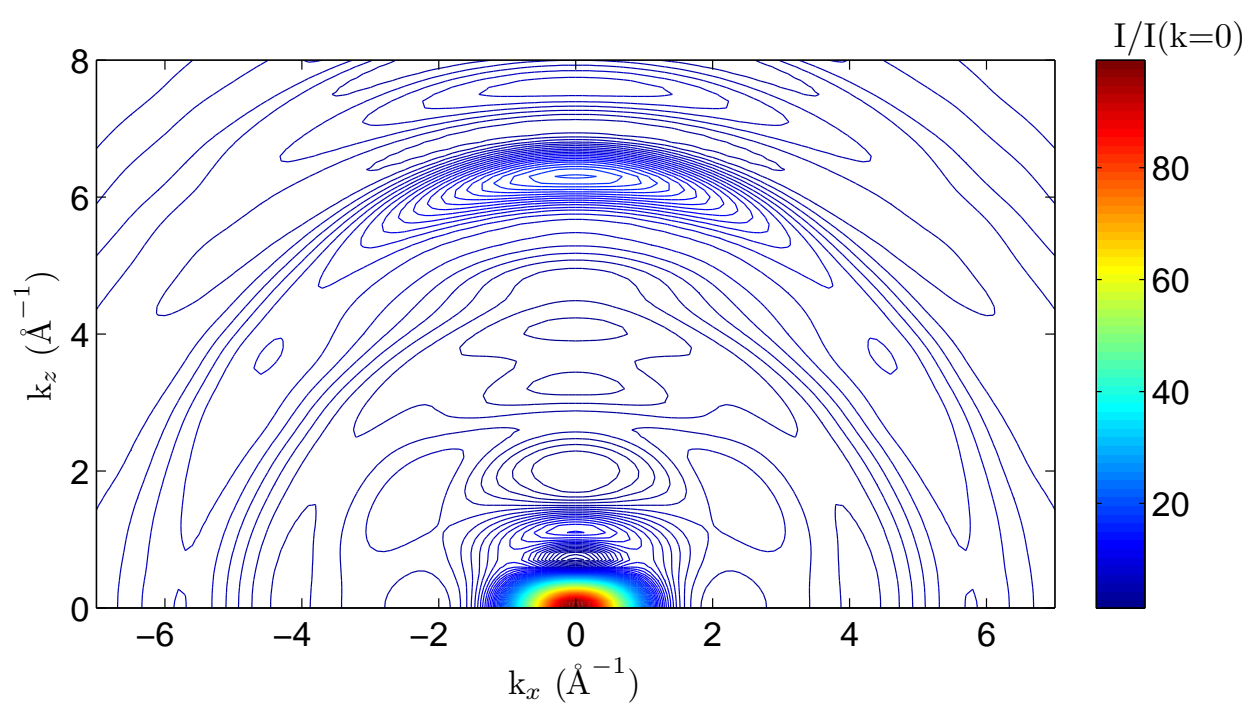

(a)

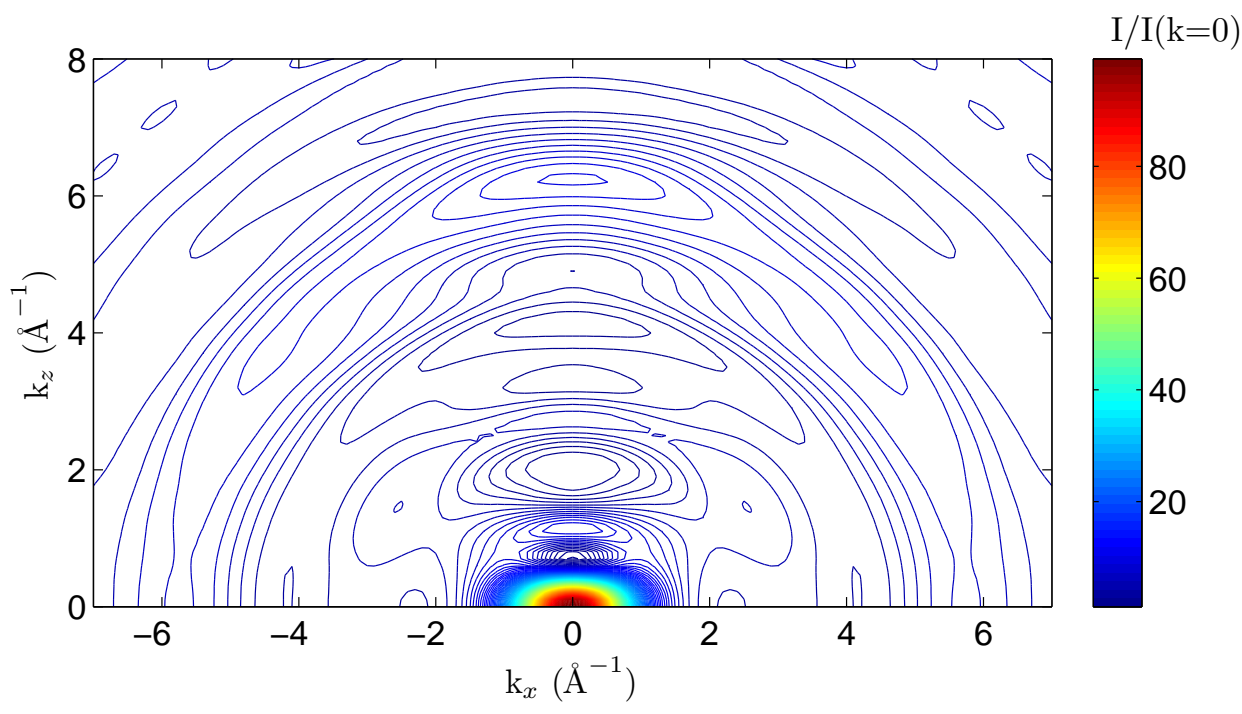

(b)

Figure 5.14. Isomerisation of stilbene; 1D scattering intensity of the geometry with the dihedral angle of a) $26^{\circ}$ b) cis, with an uncertainty in alignment $20^{\circ}$; Color scale in $\%$ of the scattering intensity at $k=0$

amorphous sample. In comparison to the amorphous sample data, the the aligned data shows resolution enhancement especially in the geometry range between the cis conformation and conformation with the dihedral angle of about $120^{\circ}$. This 
practically means that a time-resolved measurement has much better prospects when measuring 1D aligned samples. 

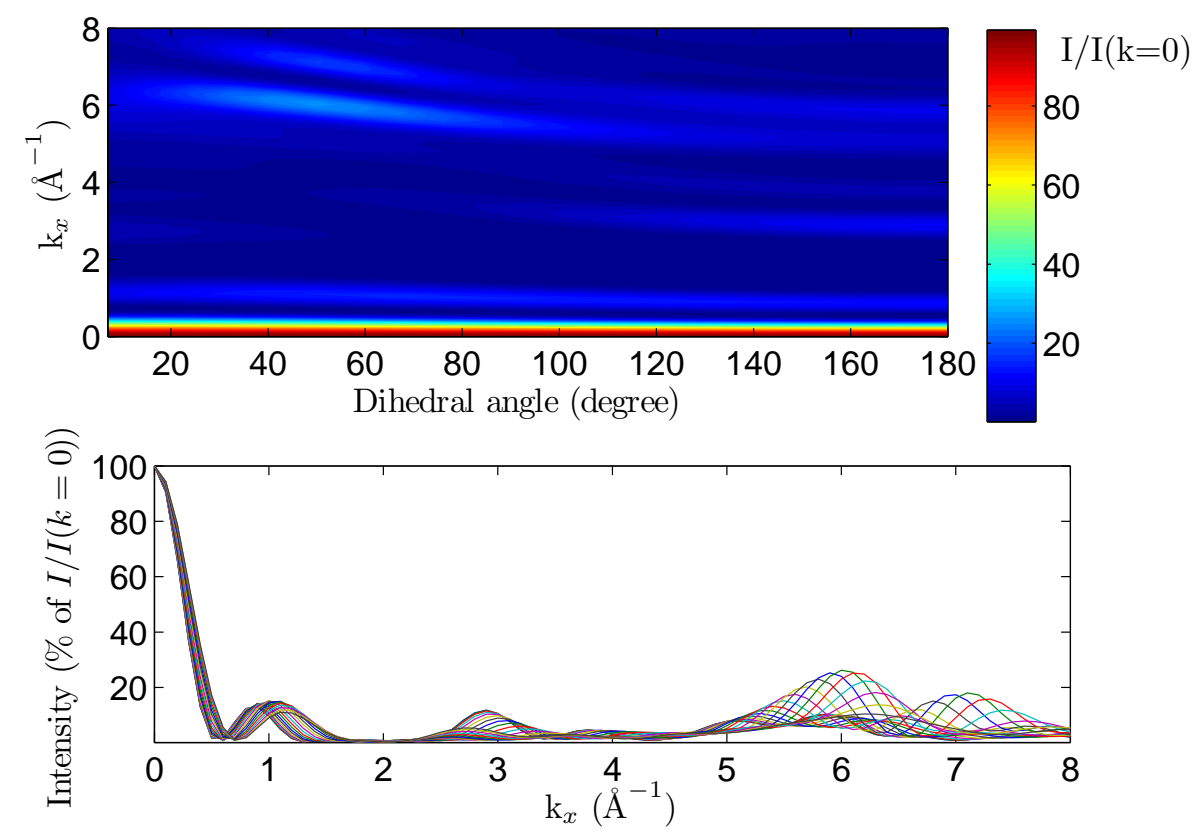

(a)
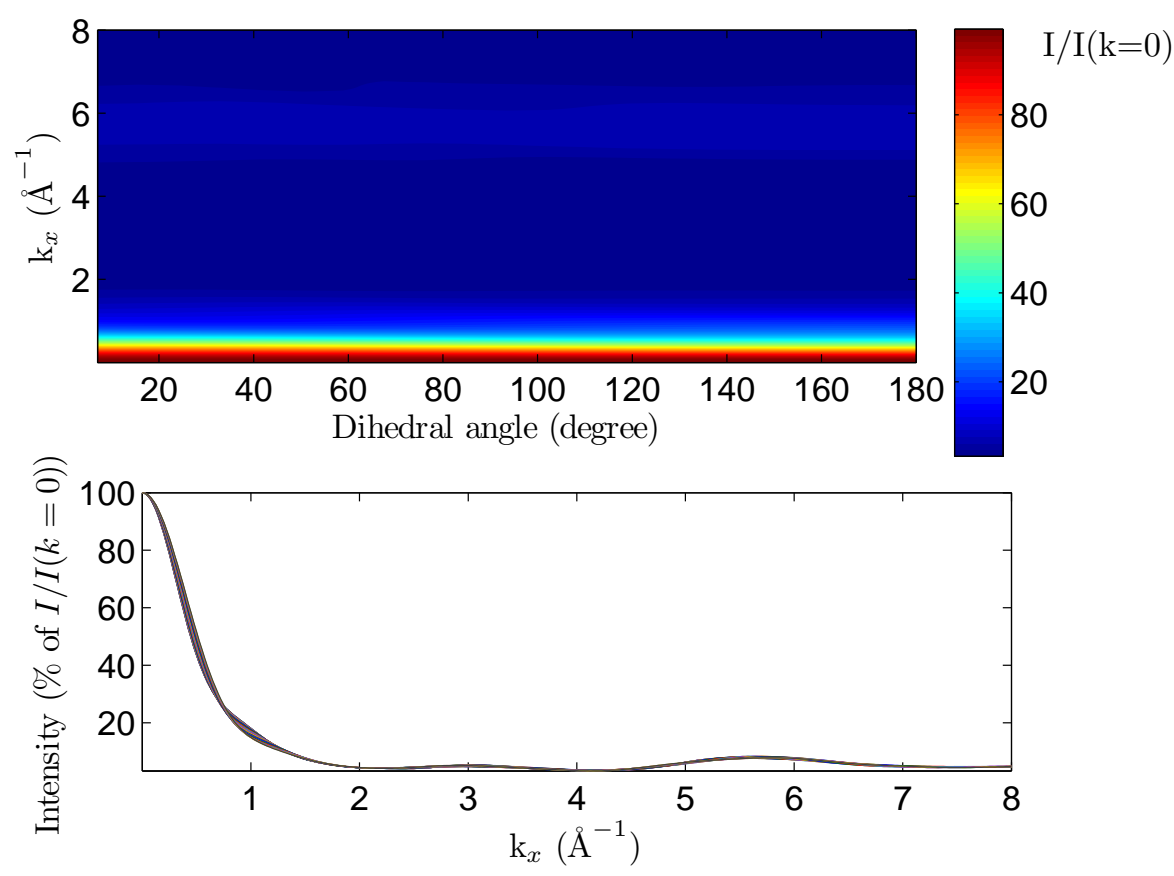

(b)

Figure 5.15. Total intensity during the isomerisation of stilbene with a) 1D alignment, b) randomly oriented ensemble 

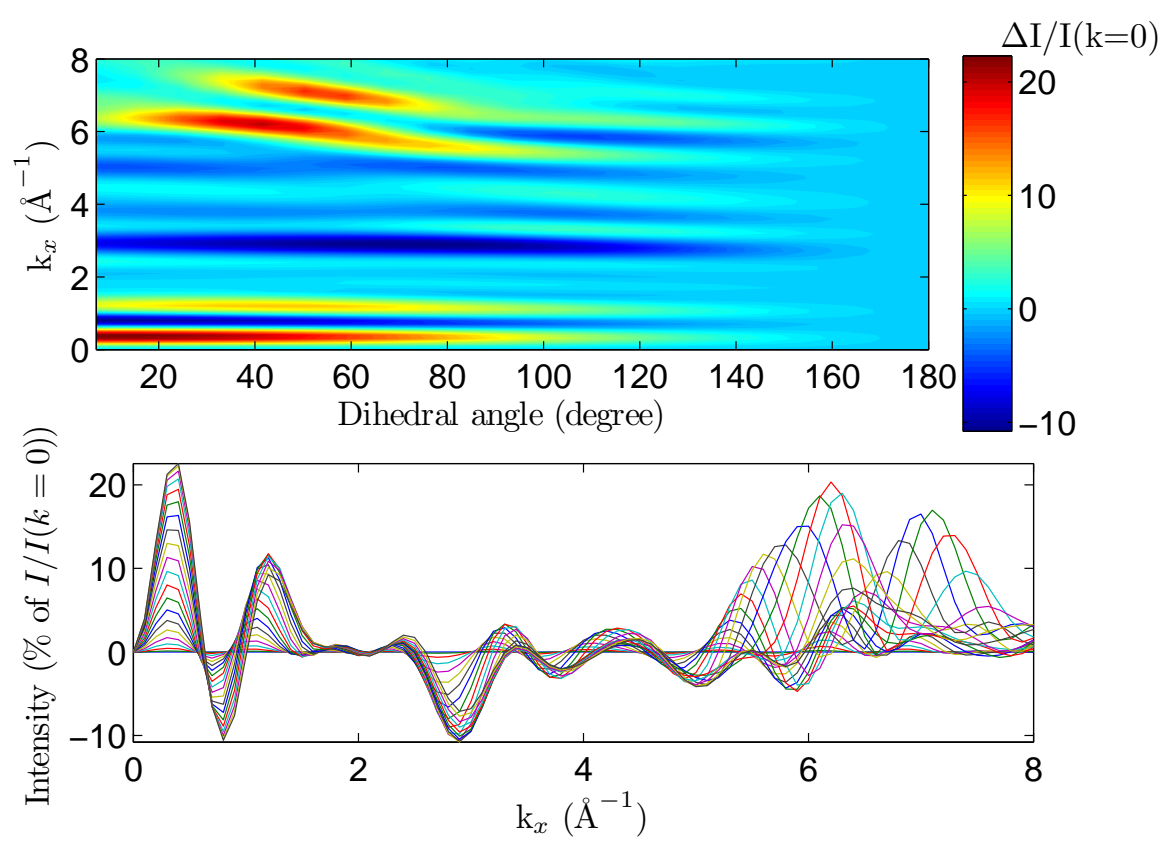

(a)
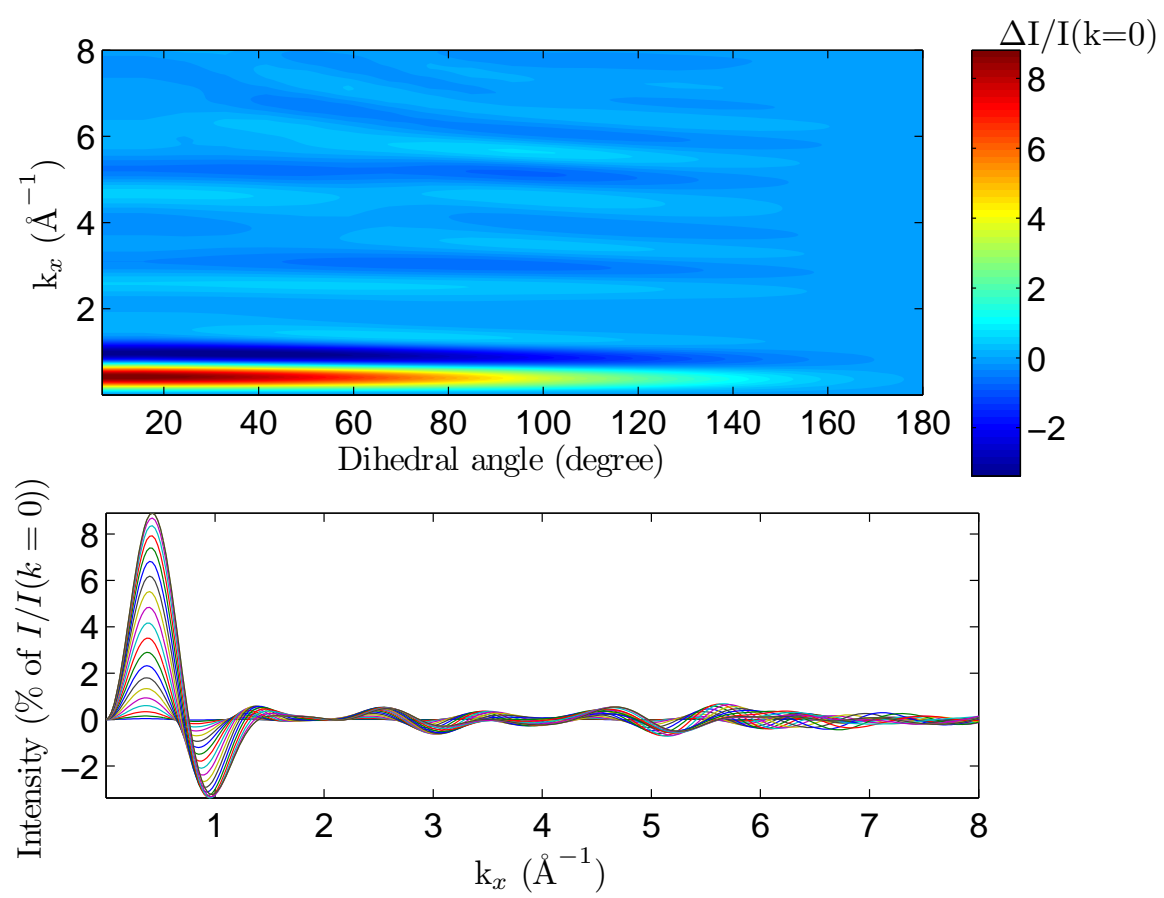

(b)

Figure 5.16. Intensity difference between the intensity of trans stilbene and a geometry with a given dihedral angle with a) alignment, b) randomly oriented ensemble 

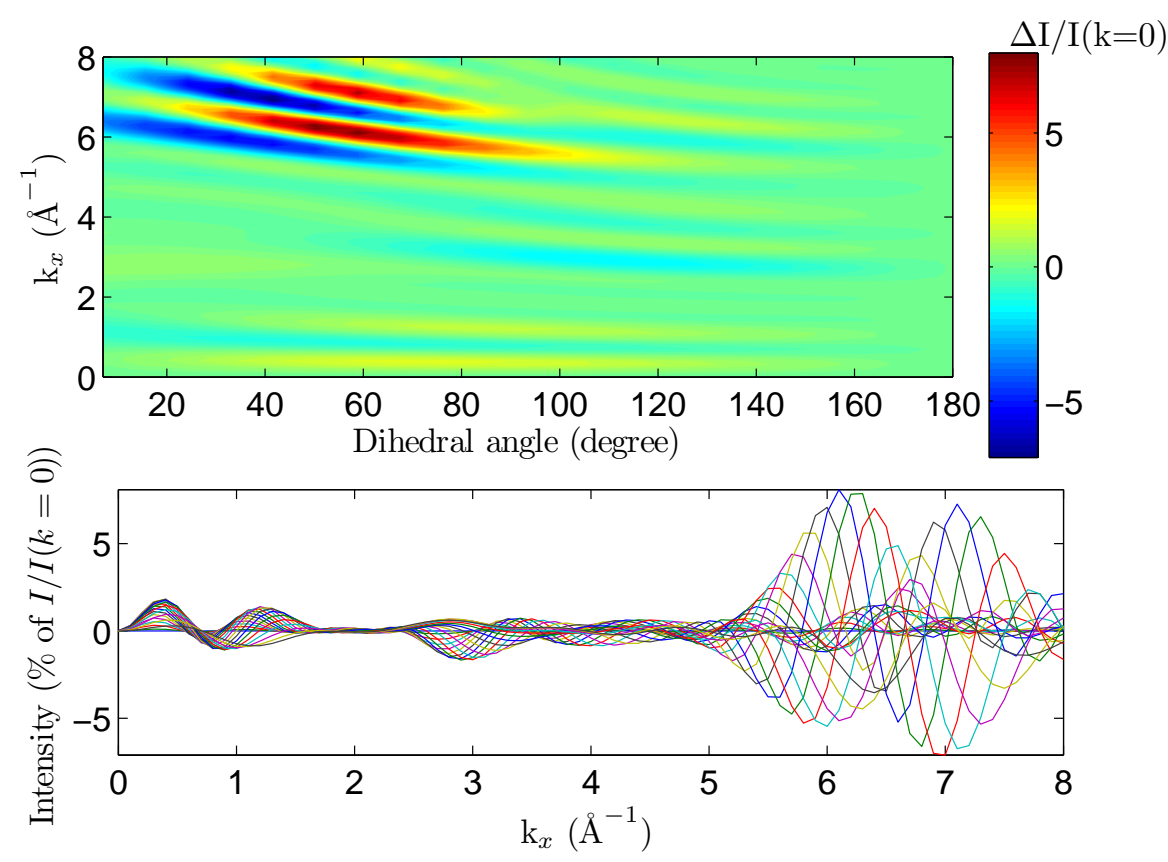

(a)
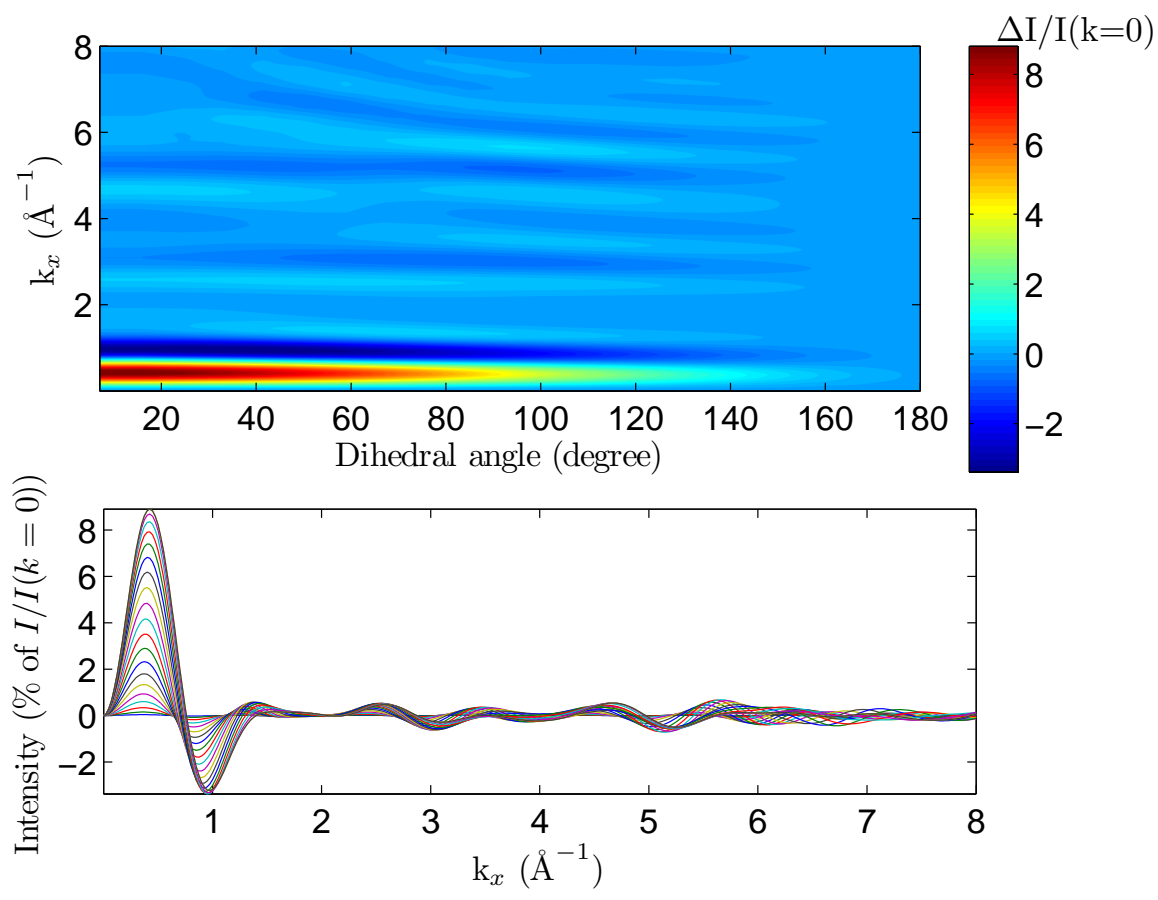

(b)

Figure 5.17. Intensity difference between two following geometries, with a 10 degree dihedral angle step, with a) alignment, b) randomly oriented ensemble 


\subsection{Conclusions}

The one dimensional alignment offers significant improvement to the structural information provided by the randomly oriented sample. While the three dimensional alignment is experimentally very demanding, especially having in mind the necessary concentration of the aligned molecules, the 1D alignment is slightly less of a challenge and so a good candidate especially in the area of time dependent scattering measurements.

Since the 1D alignment offers improved scattering intensity information it opens the opportunity for better time-resolved x-ray scattering measurements. At the same time, although it is sensitive to the uncertainty in the alignment angle, it offers good results even at a realistic alignment uncertainty distribution of $20^{\circ}$ full width at half maximum.

It is necessary to look at the importance of the alignment axis on the scattering spectra. In this test case the alignment axis was chosen to be the main axis of the moment of inertia of the molecule. This is just a test axis used to study the alignment properties. In reality the alignment axis is the main polarizability axis. As the molecular geometry changes in the isomerisation process the alignment axis changes accordingly, which virtually enhances the possible information provided by the scattering signal. As we mentioned before, the spectrum of the 1D aligned system basically provides the information about the electron density projected onto the alignment axis. Therefore, theoretically, by changing the alignment axis one could choose the projection with most pronounced density change during the observed process. If we introduced suitable methods of tagging and determining the alignment axis this would gives us the opportunity to study the molecule with respect to different axis and so possibly focus on the electron density area of interest.

The possible tree-dimensional alignment and orientation would bring the scattering experiment closer to single molecule scattering conditions. However, this is a much more difficult task for the experiment, while as we have seen, the 1D alignment significantly improves the scattering resolution between geometrically close structures. This could contribute to vital enhancement of the time-resolved structural dynamics scattering measurements. 


\section{Chapter 6}

\section{Water in Strong Soft X-Ray Laser Fields}

\subsection{Introduction}

So far in this thesis we have studied different aspects of x-ray scattering at low x-ray intensities. As the x-ray source becomes more intense, the energy transfer to the sample becomes more prominent. Strong laser pulses, such as those from the free electron laser (FEL), reach pulse intensities up to $10^{16} \mathrm{~W} / \mathrm{cm}^{2}$. For comparison the static electric field between proton and electron in the hydrogen atom has a field strength of the same order of magnitude. At these fields the energy transfer from the x-ray field to molecules or clusters in the sample becomes so great that, on a very short time scale, due to single- and multi-photon excitations, the sample transforms to a collection of highly charged ions embedded in a quasi free electron gas. The formation and dynamics of this state is called a Coulomb explosion. Necessarily such process influences any measurement performed on the sample in a number of ways. The description and study of these is the objective of this Chapter.

The process of Coulomb explosion can be generally divided into tree main phases. In the first phase the laser light couples to the atoms as if they were isolated, the cluster environment has no effect. This phase is dominated by photoionizations. In the second phase the cluster expands due to the repulsion between 
ions that were created in the first phase, together with 'inner ionized', 'quasi free' electrons - highly energetic electrons that are still bound to the cluster. In the third phase the energy redistributes and recombinations occur.110111

In structural measurements, radiation damage has set a limit for the probe pulse intensity that a sample can be exposed to. For example, in the case of x-rays of $12 \mathrm{keV}$ the limit is 200 photons per $\AA^{2}$. [4 The emergence of $\mathrm{x}$-ray sources with high brilliance has led to proposals of single molecule structural measurements that would highly exceed this damage threshold. At the intensity levels proposed for single molecule coherent diffraction, the effect of Coulomb explosion is inevitable. A way to avoid the problem with sample disintegrating in the course of structural measurement is theoretically simple - the probe pulse must be intense and short enough to provide structural information before the structural changes, due to the Coulomb explosion, erode the collected scattering data. $112|113| 114 \mid 115$

A number of theoretical works ${ }^{116[117 \mid 118}$ have studied the effect of Coulomb explosion in connection with single molecule diffraction. Neutze et al in the Reference [5] presents simulations of intense x-ray pulse-protein sample interaction and the effects on coherent diffraction. The radiation damage is described stochastically, based on probabilities for photoelectric or inelastic event. The behavior of the test system - molecule of T4 lysozyme including its 118 crystallographically determined water molecules - was described trough molecular dynamics, with force fields incorporating Morse potentials for the description of all chemical bonds. Using this approach it was shown that $12 \mathrm{keV}$ pulses with intensities of $10^{11}$ to $10^{13}$ photons per $\AA^{2}$, on the order of up to tens of femtoseconds long, can be theoretically used for single molecule diffraction before the onset of Coulomb explosion renders the measurement meaningless. However, this approach omits the screening effect provided by the free electron gas that builds up in the sample during the irradiation. It also neglects the effects on the molecular bonds from the changes in electronic state of the system.

In the publication by Bergh et al ${ }^{119}$ the x-ray laser-matter interaction model is expanded by accounting for the free electron gas through iterative use of the Poisson-Boltzmann equation. This electron gas created by Auger and secondary electrons is assumed to have the simple Maxwell-Boltzmann distribution. The test systems in this case were relatively large water clusters, containing 660 molecules and 1320 molecules respectively. It was shown that the presence of the electron 
gas slows down the Coulomb explosion. A radiation-hydrodynamic model for the interaction of a soft x-ray laser pulse with matter was studied in the Ref. [120.

A detailed model of laser-matter interaction can be provided by time dependent density functional theory in its form beyond linear response (for details on DFT and TDDFT see Chapter 2.3). Since this ab-initio approach is computationally expensive, the size of the studied system has to be accordingly limited. The $\left(\mathrm{H}_{2} \mathrm{O}\right)_{8}$ water cluster with $\mathrm{S}_{4}$ symmetry was chosen as a test system (see Figure 6.1) in our calculations. It is small enough to minimize the computational costs of TDDFT, while the geometry of this cluster allows for a limited level of volume effects, such as free electron gas shielding which will be described later in this Chapter. This water octamer is notable because of its tricoordinate $\mathrm{H}$-bond deficient water structure. It has been an object of both theoretical ${ }^{[121}$ and experimental ${ }^{[122}$ studies. The two energetically lowest water octamer conformations form a cube with the water molecules positioned in the corners, oriented to form $\mathrm{D}_{2 d}$ or $\mathrm{S}_{4}$ symmetry. The tricoordinate structures are known to form water ${ }^{123}$ and ice ${ }^{124}$ surfaces, as opposed to the tetracoordinate water that dominates the bulk.

The dynamics of molecules under the influence of fields with very high intensity and very short pulse length, from the FEL fields, is by nature highly non-linear. As we described in the Chapter 2.3.3 a convenient method of describing the behavior of a system like this is the TDDFT in its form beyond linear response. The full TDDFT is a promising method offering an ab-initio approach to the problem as opposed to the widely used molecular dynamics treatment, lowing the study of small molecular systems. The coupling of the laser field to the molecular system is formulated in semi-classical way, so called minimal coupling scheme, where laser is treated as a classical field. In the used TDDFT implementation the external laser field is is described using the dipole approximation, which is always justified for optical wavelengths, while for hard x-rays it is satisfied only in the case of inner-shell electrons.

It should be noted that the TDDFT functionals used for describing the electron behavior in strong fields are only in the development stage. Therefore experiments, such as the time-dependent scattering measurements, may prove invaluable for gaining better understanding of the behavior of electronic structures in strong fields and help improve the precision of the computational methods and TDDFT functional development. 


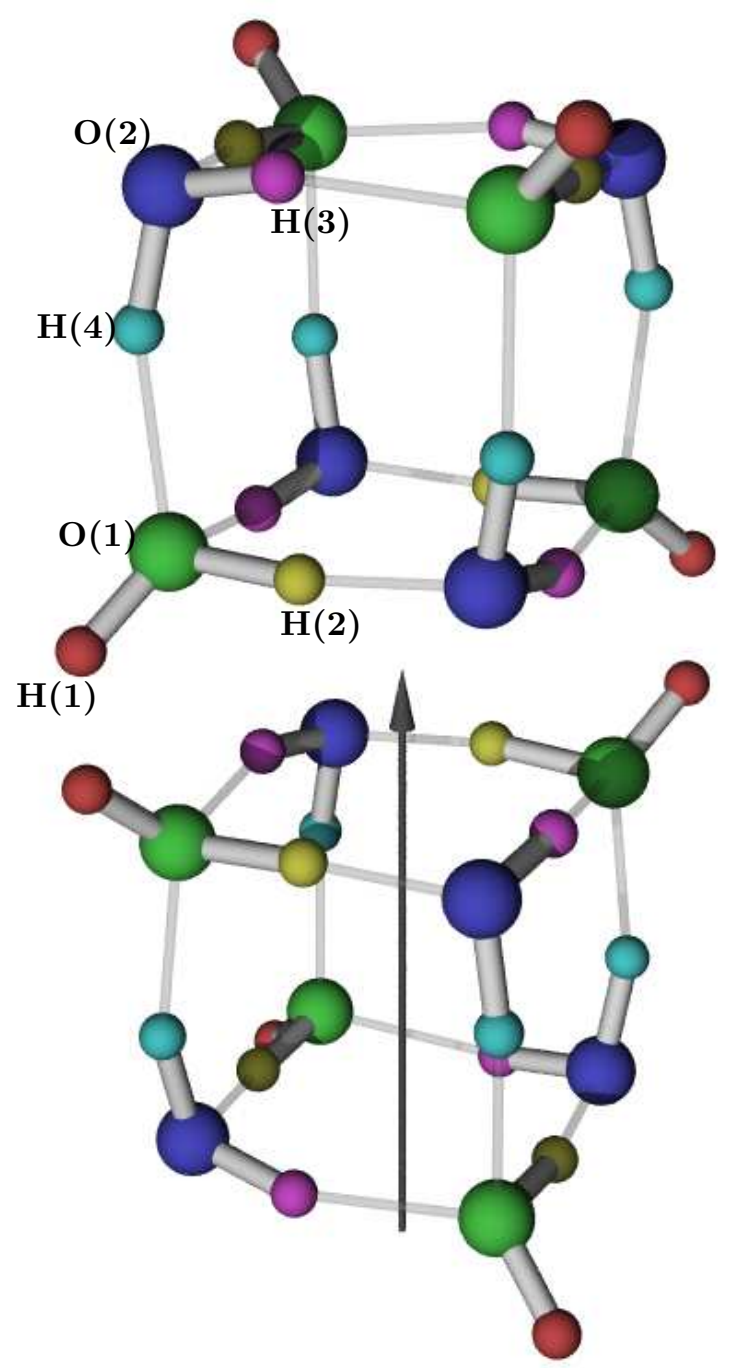

Figure 6.1. Water octamer with symmetrically independent atom groups colored separately. The arrow shows the axis of the $C_{4}$ rotation, which followed by a reflection in the horizontal plane is the generator of the $S_{4}$ symmetry group of the cluster

An important question for the structural measurements with strong x-ray laser pulses is how the behavior of the Coulomb explosion changes with the field strength. As the pulse lengths generally considered are on the order of tens of fs to $100 \mathrm{fs}$, the changes of the structure in this time should stay small if a strong, well defined scattering signal is to be obtained. The forming free electron gas has 
a dominant effect on the structural data deterioration. TDDFT is a convenient method for the description of this electron gas formation in detail.

In the following we study the Coulomb explosion behavior with respect to four different field intensities. The strongest studied intensity of $10^{16} \mathrm{~W} / \mathrm{cm}^{2}$ causes a very fast Coulomb explosion with significant changes to the structure even on the short computed time interval of 5 fs. For a comparison three weaker fields with the strengths of $10^{13} \mathrm{~W} / \mathrm{cm}^{2}, 10^{14} \mathrm{~W} / \mathrm{cm}^{2}$ and $10^{15} \mathrm{~W} / \mathrm{cm}^{2}$ are considered as well.

\subsection{Computational Details}

For the treatment of matter interaction with strong laser fields in the framework of TDDFT we must resort to solving the time-dependent Kohn-Sham equations explicitly. This approach has been implemented in the quantum chemistry program package OCTOPUS ${ }^{281125}$ in the manner shortly described in the following.

In the OCTOPUS program the nuclei are treated classically using the Ehrenfest molecular dynamics. The system is so represented as two coupled dynamical systems consisting of the system of non-interacting particles - electrons - described by the Kohn-Sham equations and classically described system of ions. The number of active electrons is reduced by using norm-conserving pseudopotentials to describe the core electrons. The time-dependent Kohn-Sham equations are solved by integration in real time with all quantities discretized on a grid in real space. The electromagnetic laser field is described classically as an additional external potential and the calculation is limited to the dipole approximation. For the laser field we then have:

$$
v_{\text {laser }}=E_{0} f(t) \sin (\omega t) \hat{\mathbf{p}} \cdot \mathbf{r}
$$

with the pulse intensity $E_{0}$, envelope of the pulse $f(t)$, polarization of the light $\hat{\mathbf{p}}$ and frequency $\omega$.

The OCTOPUS program package has been successfully applied in a number of previous studies which has been published in literature. For example in the paper by Isla [126] the Coulomb explosion and fragmentation of deuterium clusters $\mathrm{D}_{13}^{+}$ has been studied.

A TDDFT study of Coulomb explosion of $\mathrm{D}_{2}$ by Livshits and Baer [127] compares three different approaches: the time-dependent Hartree-Fock method, the the simplest TDDFT method - so called ALDA which stands for adiabatic lo- 
cal density approximation and TDDFT with the Baer-Neuhauser functional 128 . This work concludes that the Baer-Neuhauser functional, which does not suffer from long-range self-repulsion while incorporating ground state correlation effects similar to ALDA, does not offer any significant improvement to ALDA.

Because of the fact that the OCTOPUS program uses a pseudopotential description for core electrons the studied laser wavelength has to be above the oxygen 1s absorption edge which is $4 \mathrm{~nm}$. We chose the soft x-ray wavelength of $10 \mathrm{~nm}$, corresponding to a photon energy of $120 \mathrm{eV}$, which is already reachable with the FLASH-FEL in Hamburg. This means that all valence electrons are in principle allowed for possible excitations in the simulation. It also keeps us well within dipole approximation considering the size of the cluster. The time step of the simulation is 0.1 atto-seconds.

We chose the ALDA approach for our calculations. Although it suffers from well known shortcomings, such as self-interaction effects, it has an advantage of being a simple, extensively studied model. We note that the well known problems of linear response TDDFT in describing charge transfer excitations are due to the perturbative treatment used in that approximation,, 129 and are not expected to appear in the present calculation, which goes beyond perturbation theory by employing an explicit time integration.

There is a number of important distinction between the hard x-ray and soft x-ray laser-matter interaction. The higher energy end of the absorption spectra of water is dominated by the two main lines - the $\mathrm{L}$ edge at about $30 \mathrm{eV}$ and the $\mathrm{K}$ edge at about $530 \mathrm{eV} \stackrel{1301131}{ }$ Table 6.1 shows few main x-ray scattering cross sections for oxygen. Here it can be seen that the main contribution to the radiation damage at $100 \mathrm{eV}$ comes from the photoionization. The dominant photoelectric cross section drops four orders of magnitude between $100 \mathrm{eV}$ and $10000 \mathrm{eV}$ having a significant impact on total scattering cross section decrease for hard x-rays.

Table 6.1. Photoelectric, elastic and inelastic scattering cross sections of oxygen at different photon energies in barns/atom, taken from Reference [132.

\begin{tabular}{rccc}
\hline energy $(\mathrm{eV})$ & Photo & Elastic & Inelastic \\
\hline 100 & $1.6510^{6}$ & 42.5 & $2.8310^{-3}$ \\
1000 & $1.2610^{5}$ & 39.9 & $2.2910^{-1}$ \\
10000 & $1.4710^{2}$ & 6.85 & 3.44 \\
\hline
\end{tabular}

An important physical characteristics with respect to the radiation damage is 
the inelastic electron scattering cross section. With hard x-ray photon pulses the main contribution to electron scattering events comes from Auger electrons,, 133 as the cross section for the scattering events with the high energy photo-electrons is very small. The photo-electrons are expected to leave the sample very fast. In the case of soft x-ray pulses, the photo-electrons themselves have energies in the range where electron scattering cross section is high and so contribute to the 'inner ionized' electron gas formation. It is however important to note that for strong fields these cross-sections have to be modified to take multi-photon effects into account. These effects are included in the present calculation, since the field is explicitly included in the simulation (in contrast to other approaches based on perturbation theory).

The simulations of the water cluster $\left(\mathrm{H}_{2} \mathrm{O}\right)_{8}$ in the strong x-ray field starts with the water octamer in its ground state structure. The starting guess for the geometry optimization of this cluster was taken from the Cambridge Cluster Database ${ }^{134}$.

The x-ray pulse in the calculations has a Gaussian shape with the total pulse length of $25 \mathrm{fs}$ in the case of the three weaker fields, and $100 \mathrm{fs}$ in the case of the strongest field. Since the calculated time interval is shorter than the pulse length the simulation starts with the pulse envelope at the maximum intensity at $\mathrm{t}=0$.

\subsection{Energy Absorption}

Within sub-femtoseconds of the application of the x-ray field the electrons start to absorb the laser energy. In the following, the electron and nuclear dynamics are presented on plots of various physical properties as a function of time.

In order to find out which orbitals are most influenced by the strong laser field, by for example electron hole creation, one has to follow the overlap between the ground state and time dependent molecular orbitals $\left|\left\langle\psi_{i}(t) \mid \psi_{i}(0)\right\rangle\right|^{2}$. Figure 6.2 shows these overlaps as a function of time in the first $2 \mathrm{fs}$, in the field of $10^{16} \mathrm{~W} / \mathrm{cm}^{2}$. In this short time the displacement of the nuclei from the starting position is smaller than one spatial grid step $(0.08 \AA)$. Up to about 1 fs the fastest rate of change belongs to the higher valence orbitals. This behavior changes after $1 \mathrm{fs}$ when with a rapid drop of overlap value for the oxygen $2 s$ orbitals, representing creation of electron holes in the corresponding orbitals. Because of symmetry effects there 


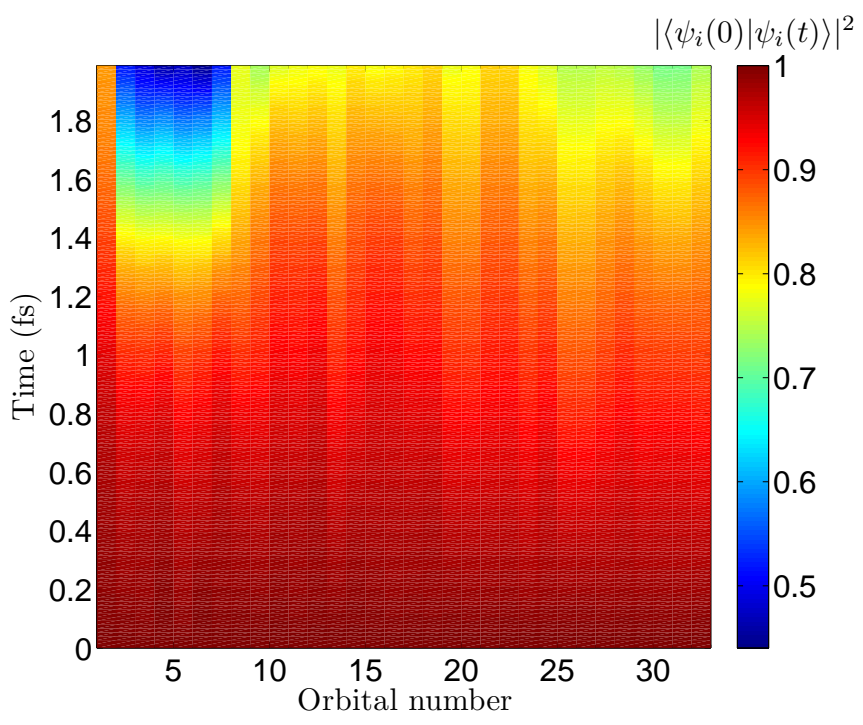

Figure 6.2. The overlap between ground state and time dependent molecular orbitals, under the laser field of $10^{16} \mathrm{~W} / \mathrm{cm}^{2}$. Orbitals are ordered by energy, from core (oxygen $2 s$ ) to valence orbitals, so that orbital 32 is the highest occupied molecular orbital

are two " $2 s "$ molecular orbitals (or rather two molecular orbitals formed from the eight oxygen $2 s$ atomic orbitals) which do not participate in the ionization process, for the given polarization of the x-ray field. These are orbital 1 and 8 in Figure6.2.

Figures 6.3 and 6.4 show the kinetic energy of the ions in the water cluster as a function of time. In Figure 6.3 the kinetic energy corresponding to the two lower laser field intensities $10^{13} \mathrm{~W} / \mathrm{cm}^{2}$ and $10^{14} \mathrm{~W} / \mathrm{cm}^{2}$ can be seen, as both fall into the same scale. In the field of $10^{15} \mathrm{~W} / \mathrm{cm}^{2}$ (Fig. 6.3p) the kinetic energy shows first signs of changes in behavior. It no longer falls into the same scale as the kinetic energies of the two lower fields, but is up to two orders of magnitude higher at the end of the studied time interval. The influence of the strongest field on the kinetic energy of the ions becomes immediately obvious from the Figure 6.4 . Although it contains the kinetic energies corresponding to all four field strengths only the one corresponding to the field of $10^{16} \mathrm{~W} / \mathrm{cm}^{2}$ can be seen, due to the fact that it is yet larger, almost two orders of magnitude, than even the kinetic energy corresponding to the field of $10^{15} \mathrm{~W} / \mathrm{cm}^{2}$. While in the weakest field the kinetic energy shows a close to linear dependence on time this significantly changes for the stronger fields. 


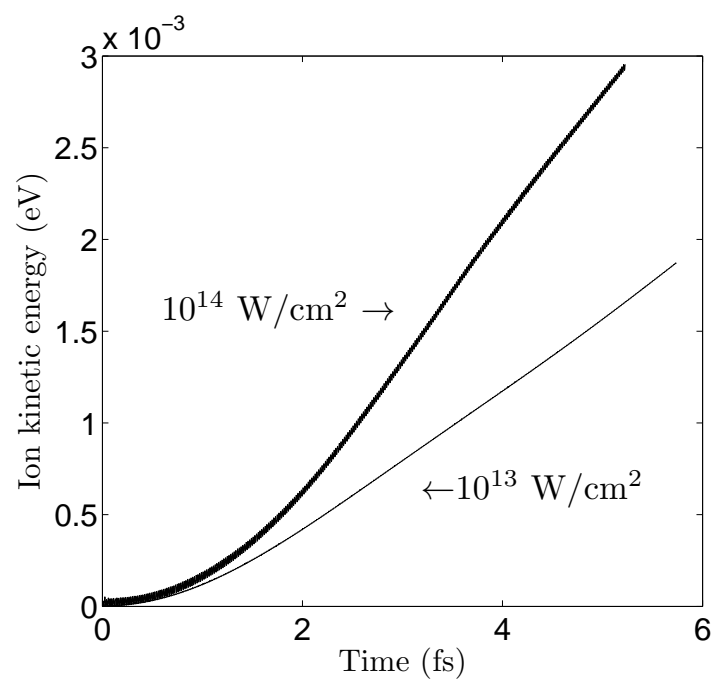

(a)

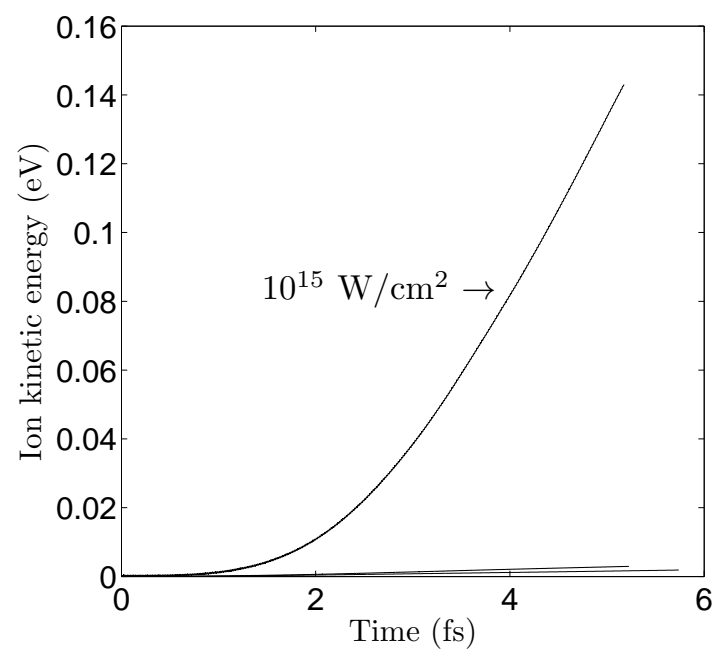

(b)

Figure 6.3. Kinetic energy of the ions under the laser field of a) $10^{13} \mathrm{~W} / \mathrm{cm}^{2}$ and $10^{14} \mathrm{~W} / \mathrm{cm}^{2}$ (thick curve) b) $10^{15} \mathrm{~W} / \mathrm{cm}^{2}$

The effect of the field strength on the ion movement can be observed also on the direct ion response to the oscillation of the laser field. The thickness of the curve corresponding to the field of $10^{14} \mathrm{~W} / \mathrm{cm}^{2}$ in Figure 6.3 a) is caused by the response of ions to the strong laser field. The small oscillations corresponding to the laser field frequency are shown in Figures 6.5a) and 6.5 b). The different 


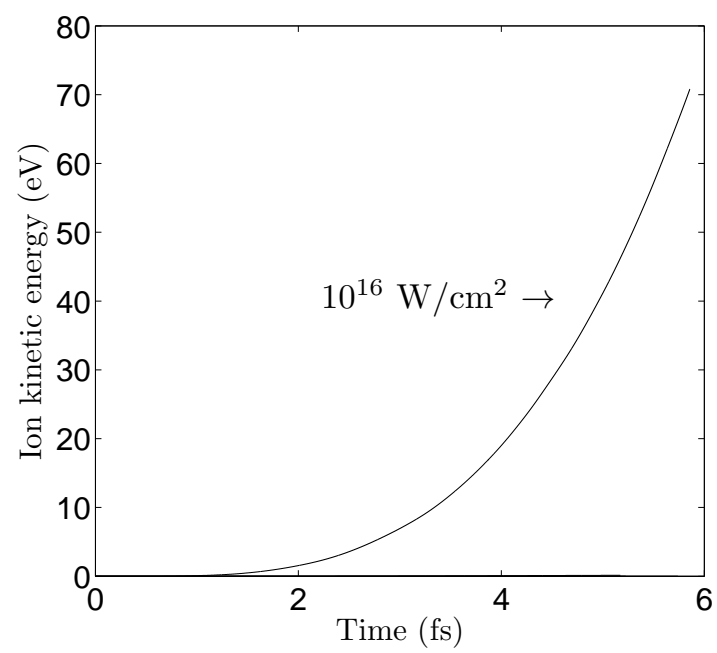

Figure 6.4. Kinetic energy of the ions under the laser field of $10^{16} \mathrm{~W} / \mathrm{cm}^{2}$

field strengths in these three cases are translated into the amplitude of the ion vibrations. For the strongest field the kinetic energy oscillation amplitude amounts to about $4.10^{-3} \mathrm{eV}$, which for comparison, corresponds to about $50 \mathrm{~K}$ thermal energy. The oscillation amplitude is then larger than the total kinetic energy response of to the two weaker laser fields, over the whole simulation time interval of $6 \mathrm{fs}$. In the case of the weaker field $10^{14} \mathrm{~W} / \mathrm{cm}^{2}$ the kinetic energy oscillation amplitude is about $4.10^{-5} \mathrm{eV}$ and so about $0.5 \mathrm{~K}$ and for the field $10^{13} \mathrm{~W} / \mathrm{cm}^{2}$ it is only $4.10^{-6} \mathrm{eV}$ or $0.05 \mathrm{~K}$.

The main part of the energy contribution to the total energy increase (see Fig. 6.8 and Fig. 6.9) comes from the electronic energy (see Fig. 6.6 and Fig. 6.7). This increase, in contrast to the kinetic energy increase, is directly proportional to the field strength, $0.6 \cdot 10^{3} \mathrm{eV} / \mathrm{fs}$ for the strongest field and about $0.6 \mathrm{eV} / \mathrm{fs}$ for the weakest, averaged over the first 5 femtoseconds. In general the energy first has to be absorbed by the electrons before it can be transferred to ion kinetic energy and cause the Coulomb explosion. 


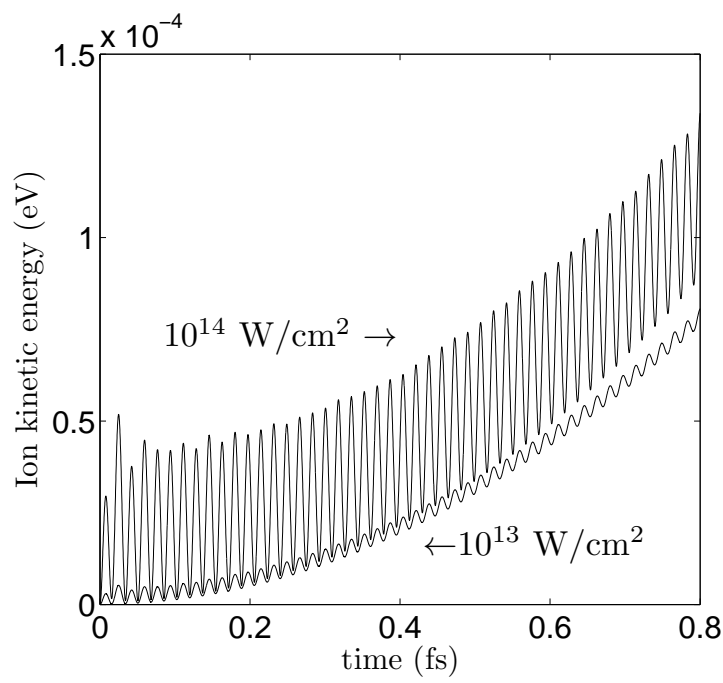

(a)

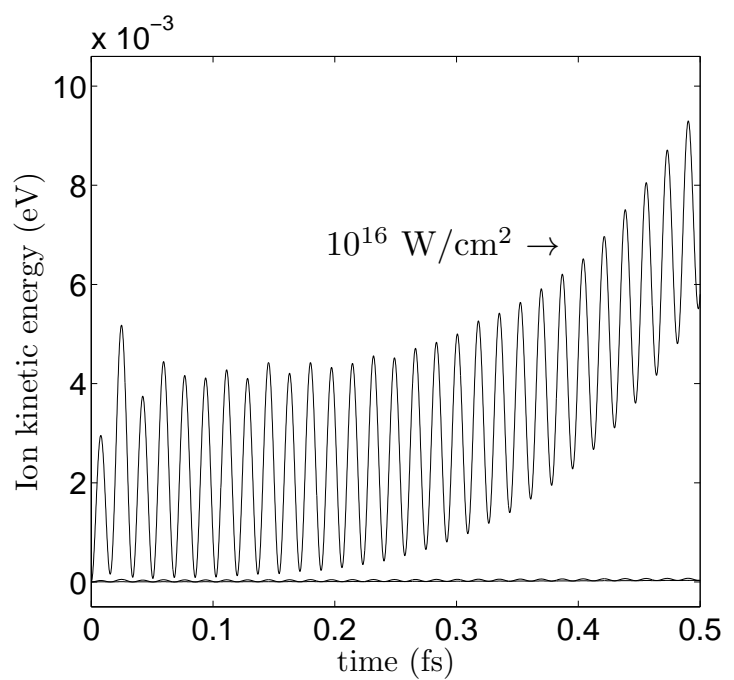

(b)

Figure 6.5. Ion kinetic energy zoom to small oscillations corresponding to the laser field oscillations a) $10^{13} \mathrm{~W} / \mathrm{cm}^{2}$ and $10^{14} \mathrm{~W} / \mathrm{cm}^{2}$ laser intensities b) all three studied laser intensities 


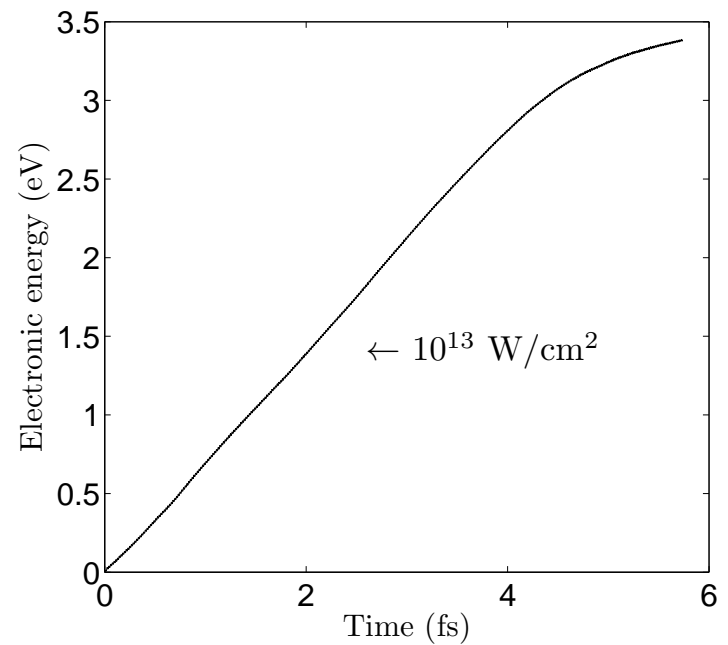

(a)

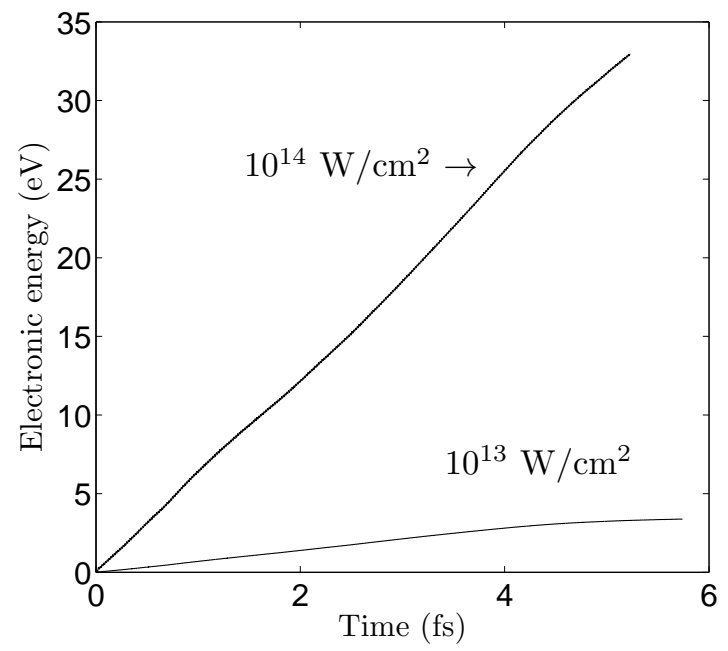

(b)

Figure 6.6. Electronic energy of the water cluster under the laser field of a) $10^{13} \mathrm{~W} / \mathrm{cm}^{2}$, b) $10^{14} \mathrm{~W} / \mathrm{cm}^{2}$ 


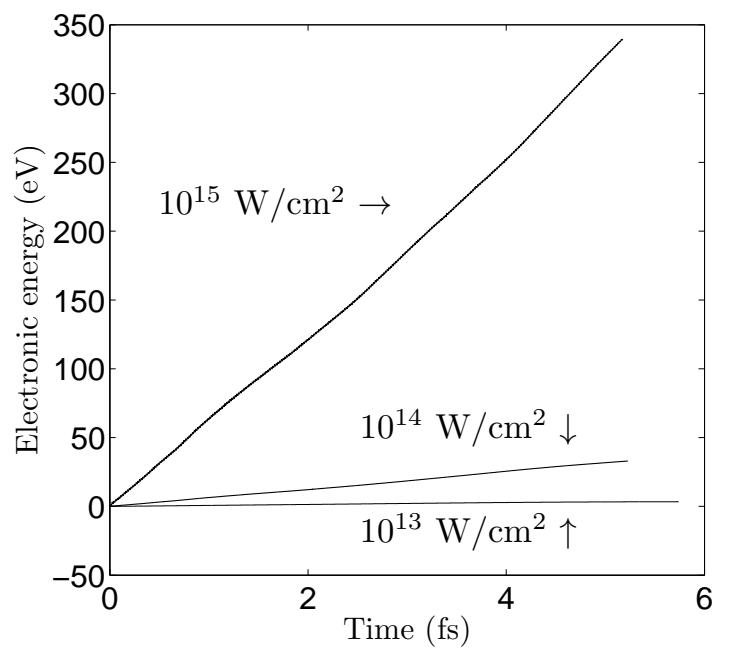

(a)

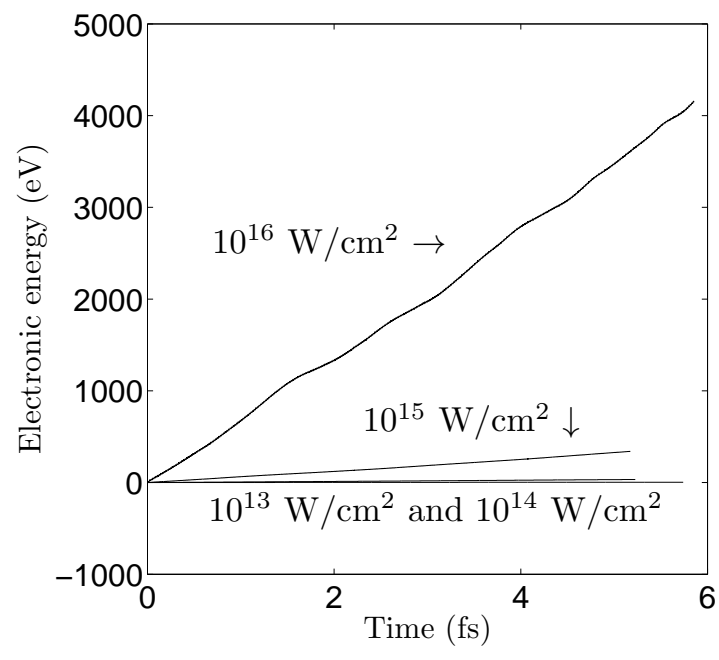

(b)

Figure 6.7. Electronic energy of the water cluster under the laser field of a) $10^{15} \mathrm{~W} / \mathrm{cm}^{2}$, b) $10^{16} \mathrm{~W} / \mathrm{cm}^{2}$ 


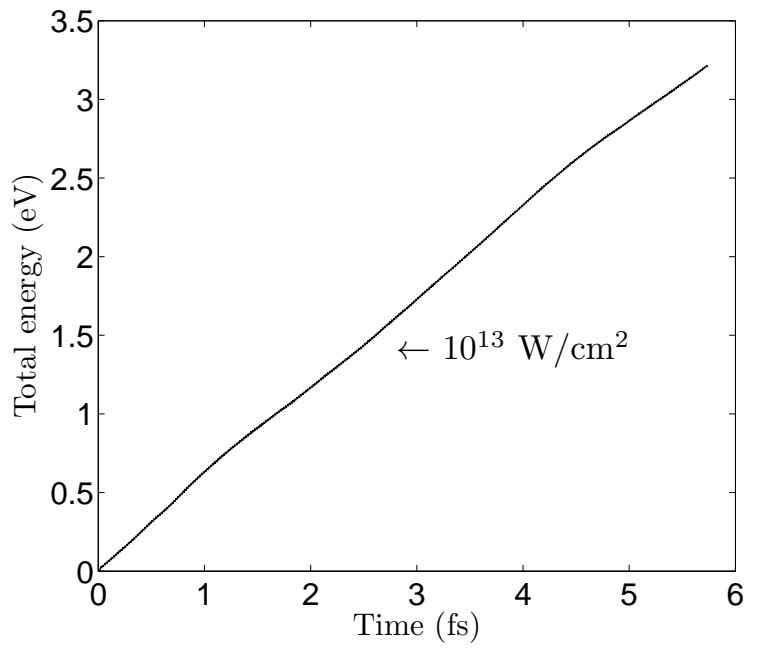

(a)

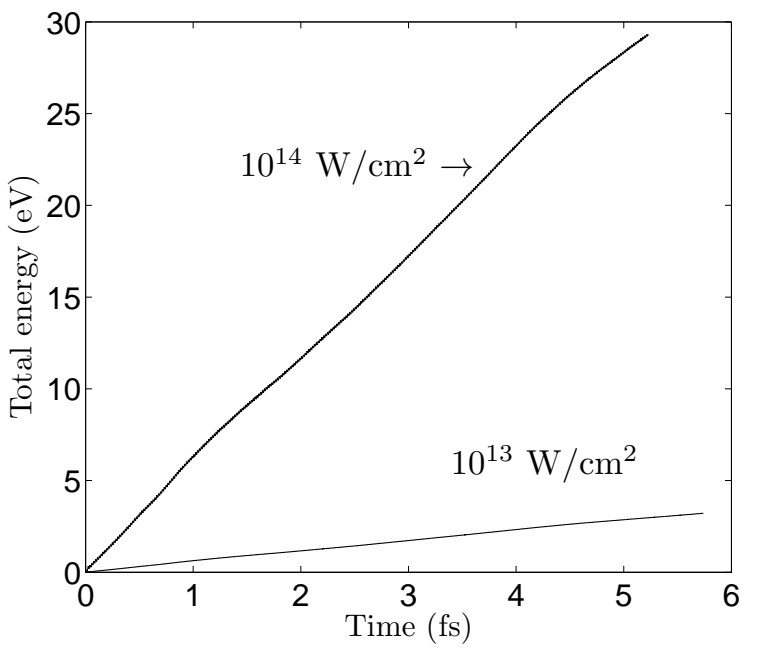

(b)

Figure 6.8. Total energy of the water cluster under the laser field of a) $10^{13} \mathrm{~W} / \mathrm{cm}^{2}$, b) $10^{14} \mathrm{~W} / \mathrm{cm}^{2}$ 


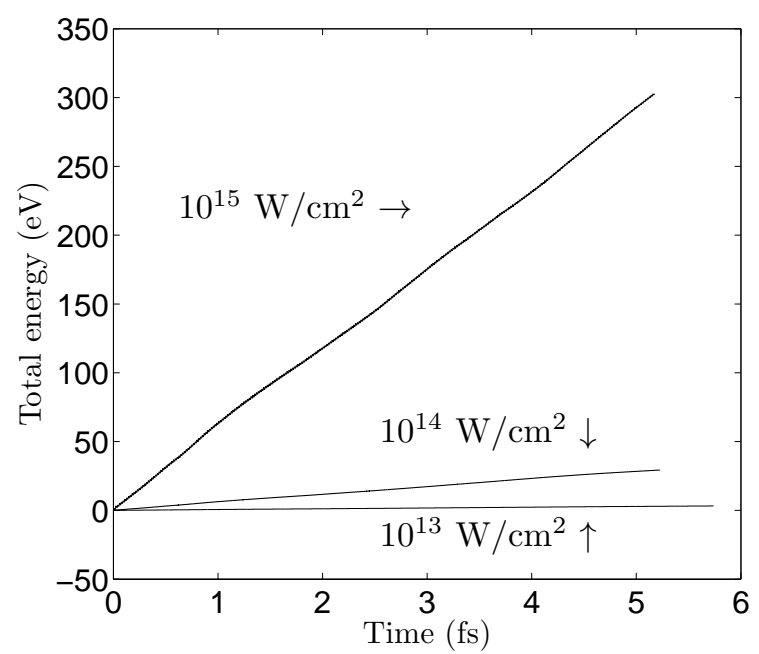

(a)

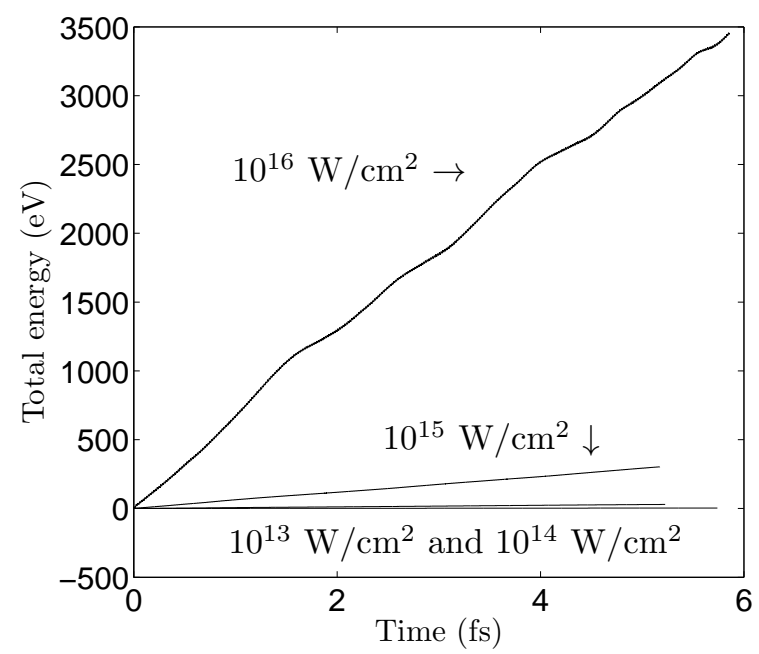

(b)

Figure 6.9. Total energy of the water cluster under the laser field of a) $10^{15} \mathrm{~W} / \mathrm{cm}^{2}$, b) $10^{16} \mathrm{~W} / \mathrm{cm}^{2}$ 


\subsection{Distances and Velocities}

The time-dependent differences of the distances of atoms from their starting positions (see Figures 6.10 and 6.11 show an interesting behavior.

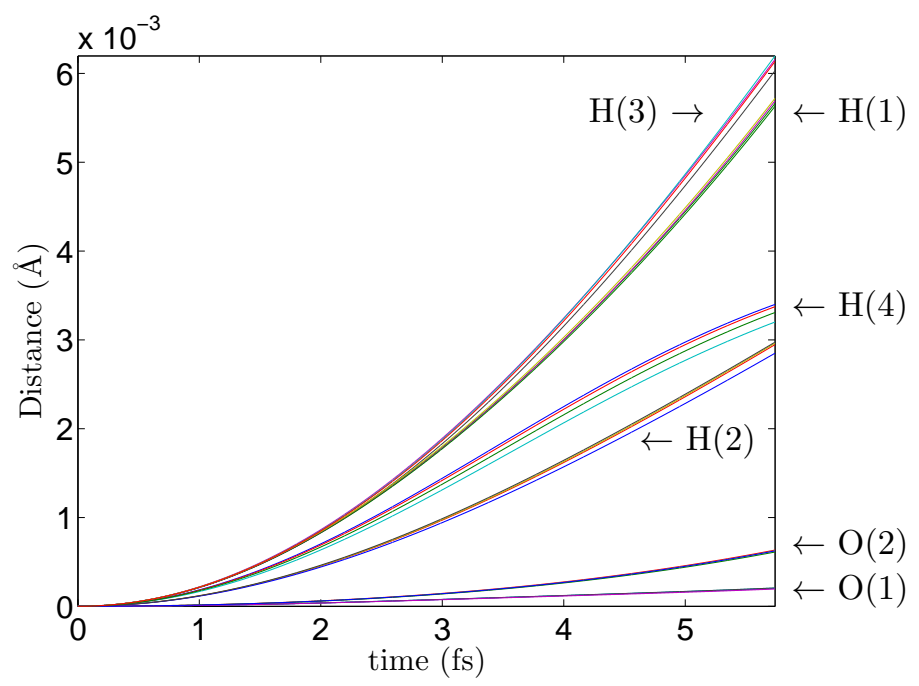

(a)

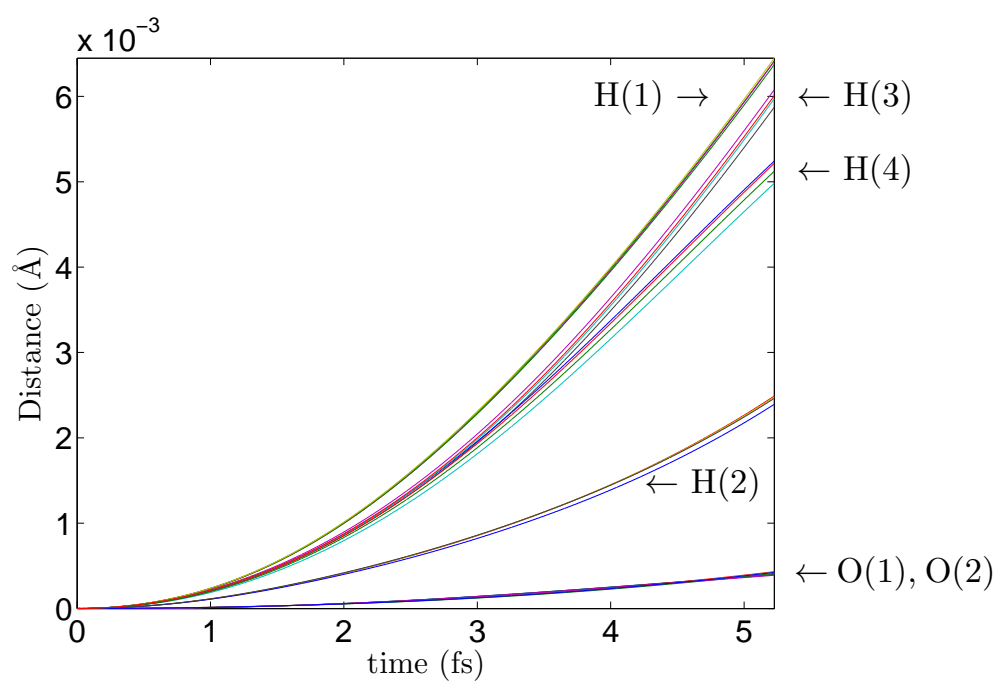

(b)

Figure 6.10. Time evolution of the atomic distances from their starting positions at the field strength of a) $10^{13} \mathrm{~W} / \mathrm{cm}^{2}$ and b) $10^{14} \mathrm{~W} / \mathrm{cm}^{2}$. Several distinct groups of atoms with similar velocities are forming. The atomic labels are introduced on the Fig. 6.1 


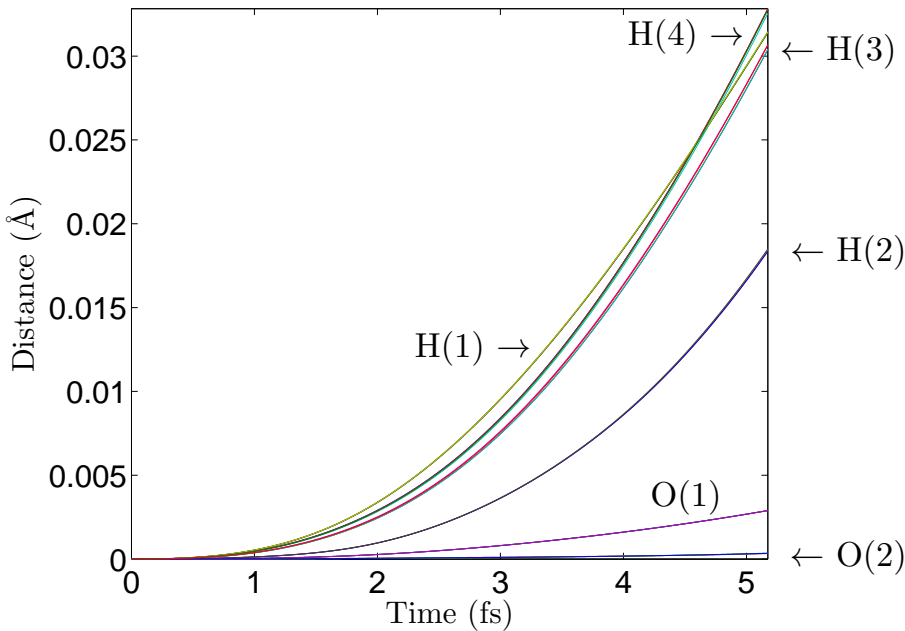

(a)

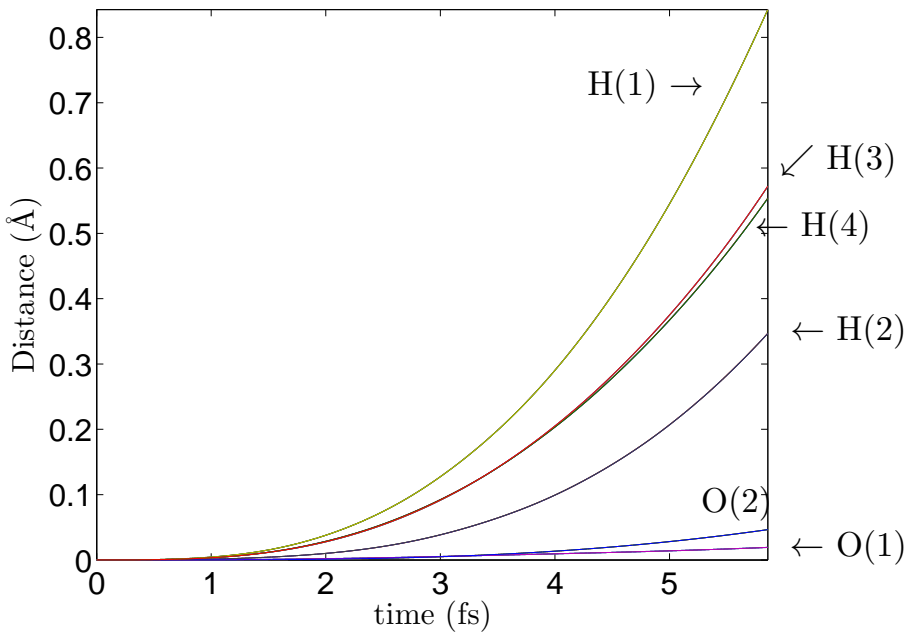

(b)

Figure 6.11. Time evolution of the atomic distances from their starting positions at the field strength of a) $10^{15} \mathrm{~W} / \mathrm{cm}^{2}$ and b) $10^{16} \mathrm{~W} / \mathrm{cm}^{2}$, with atomic group labels as in Fig. 6.1

As one can see in Figure 6.1 the water octamer has the $S_{4}$ symmetry, thus the hydrogen atoms can be organized in groups of four, depending on their position with respect to the center of the cluster. The time-dependent development of the atomic distances and velocities are in correspondence with this symmetry of the cluster. The distance plots show a number of separate bunches of curves that 


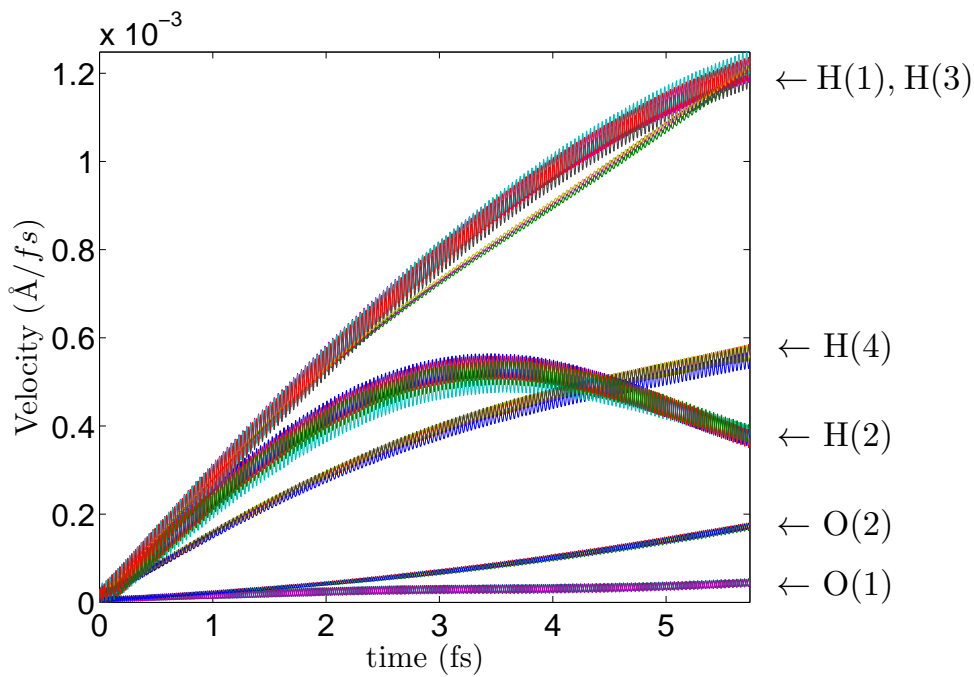

(a)

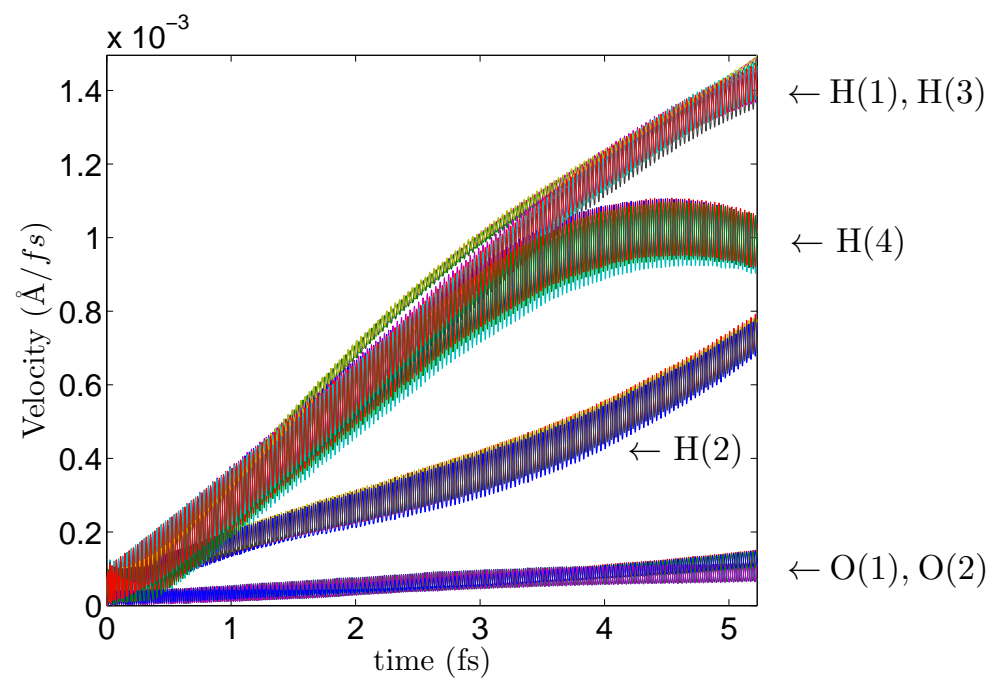

(b)

Figure 6.12. Time evolution of the atomic velocities at the field strength of a) $10^{13} \mathrm{~W} / \mathrm{cm}^{2}$ and b) $10^{14} \mathrm{~W} / \mathrm{cm}^{2}$, with atomic group labels as in Fig. 6.1 The fast oscillations correspond to the ion oscillations driven by the x-ray field

correspond to symmetrically equivalent atoms. Most notable are the four outer hydrogens, labeled as $\mathrm{H}(1)$, with the largest distance to the center of the cluster. From the point of view of velocities and displacement this group of hydrogen 


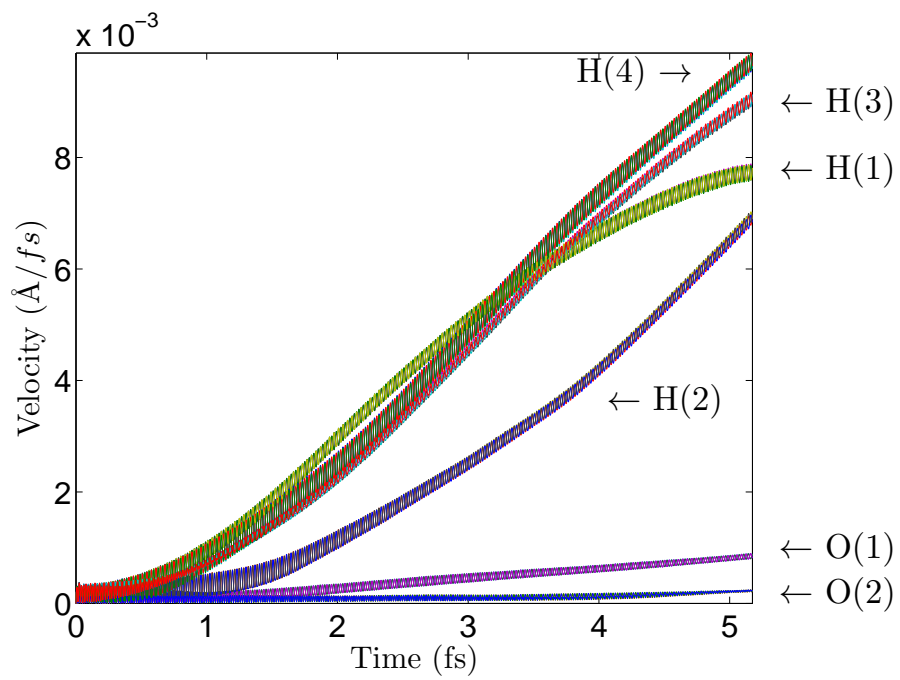

(a)

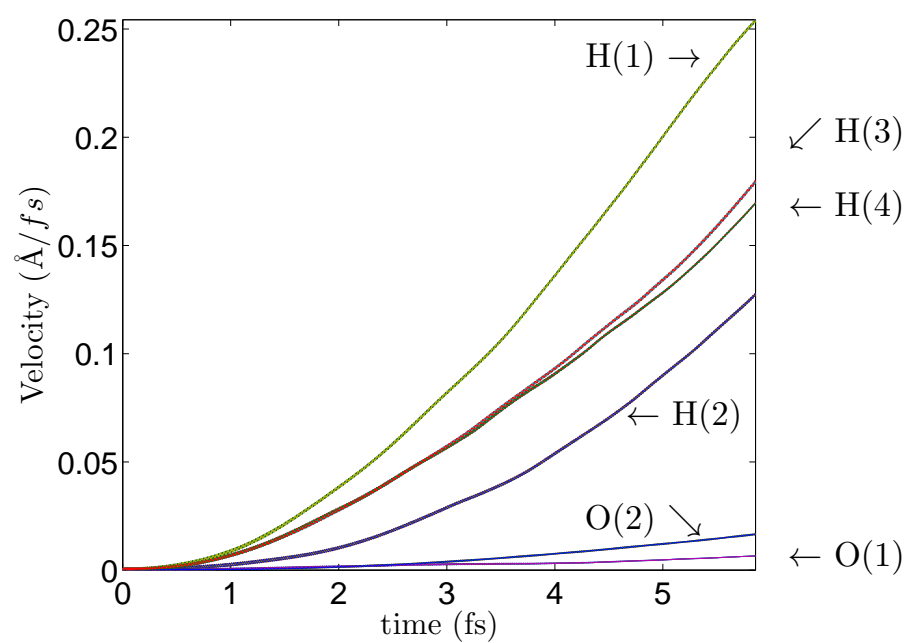

(b)

Figure 6.13. Time evolution of the atomic velocities at the field strength of a) $10^{15} \mathrm{~W} / \mathrm{cm}^{2}$ and b) $10^{16} \mathrm{~W} / \mathrm{cm}^{2}$, with atomic group labels as in Fig. 6.1

atoms is in all four fields either one of the fastest moving groups (for $10^{15} \mathrm{~W} / \mathrm{cm}^{2}$ on Fig. 6.13b) or the fastest moving group (in the other three cases) and so achieves the highest or second highest displacement. On the opposite side are the slowest, least moving hydrogens $\mathrm{H}(2)$ that share the same water molecule with the fastest hydrogens $\mathrm{H}(1)$. 


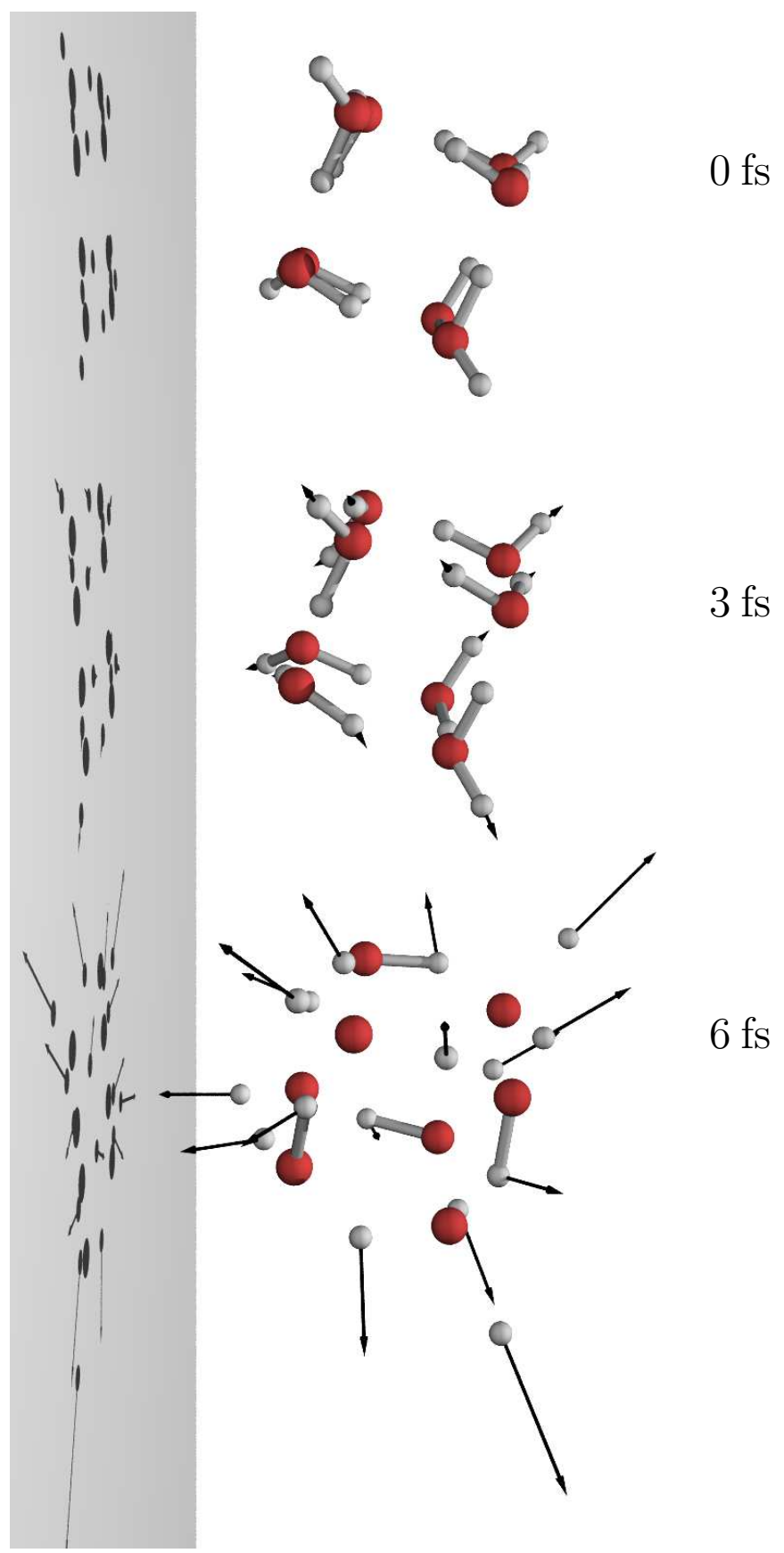

Figure 6.14. The Coulomb explosion of water cluster in the field of $10^{16} \mathrm{~W} / \mathrm{cm}^{2}$ with velocity vectors 
Although the total distance difference for the two lower laser fields stays in both cases in the same order of magnitude they show different time-dependent structure changes. Even the second strongest field causes only small atomic displacements, which even at the end of the simulation time interval are below the size of the electron density spatial grid step of $0.08 \AA$. In all three cases of the weaker fields (Fig. 6.12 and Fig. 6.13b) the velocities for some of the atomic groups start to drop after some time. Only the strongest field shows fast fragmentation corresponding to the Coulomb explosion with the distance change of the outermost hydrogens reaching $0.85 \AA$ in the first 6 fs. In this case there is no hint of any velocity drop over the whole simulation time interval.

Table 6.2. Displacement estimates based on the final velocities in the simulation

\begin{tabular}{cccccc}
\hline Field & & \multicolumn{4}{c}{ Displacement(in $\AA$ ) after: } \\
\cline { 3 - 6 } $\mathrm{W} / \mathrm{cm}^{2}$ & & $10 \mathrm{fs}$ & $25 \mathrm{fs}$ & $50 \mathrm{fs}$ & $100 \mathrm{fs}$ \\
\hline \hline $10^{13}$ & $\mathrm{O}$ & $0.0004-0.0014$ & $0.001-0.0041$ & $0.002-0.0085$ & $0.004-0.017$ \\
& $\mathrm{H}$ & $0.0048-0.011$ & $0.011-0.030$ & $0.020-0.061$ & $0.038-0.12$ \\
\hline $10^{14}$ & $\mathrm{O}$ & $0.0007-0.0011$ & $0.0017-0.0034$ & $0.0034-0.0072$ & $0.007-0.015$ \\
& $\mathrm{H}$ & $0.0058-0.014$ & $0.016-0.036$ & $0.034-0.073$ & $0.069-0.15$ \\
\hline $10^{15}$ & $\mathrm{O}$ & $0.0014-0.0072$ & $0.0047-0.021$ & $0.01-0.043$ & $0.021-0.087$ \\
& $\mathrm{H}$ & $0.051-0.08$ & $0.15-0.23$ & $0.32-0.47$ & $0.67-0.97$ \\
\hline $10^{16}$ & $\mathrm{O}$ & $0.046-0.12$ & $0.14-0.37$ & $0.31-0.78$ & $0.63-1.6$ \\
& $\mathrm{H}$ & $0.87-1.9$ & $2.8-5.7$ & $6.0-12.1$ & $12.3-24.8$ \\
\hline
\end{tabular}

To compare the overall effect of the different field strengths we estimate the displacements after times longer than the simulation times using the velocities from the last stages of the simulation. The results are shown in table 6.2. We can see that all the three weaker fields cause only a small displacement even after a relatively long time of $100 \mathrm{fs}$, while the strong field causes very fast movement of hydrogens (2.8-5.7 $\AA$ in $10 \mathrm{fs})$. Here it should be stressed that only the strongest field exhibits the behavior of Coulomb explosion as it could be inferred from the properties that have been presented so far. At the same time the movement of oxygens is, as expected due to their mass, relatively slow even in the strong field, where in the first 25 fs we estimate the maximum displacement to only $0.36 \AA$. As the structural measurement is focused on heavier elements, and not hydrogen, even the pulses of this intensity and length of up to 25 fs can lead to relatively good results. However longer timescales, 50 to 100 fs adversely affect even the 
heavier oxygen causing displacements of $0.8 \AA$ to $1.6 \AA$.

\subsection{Coulomb Explosion and Electron Density Change}

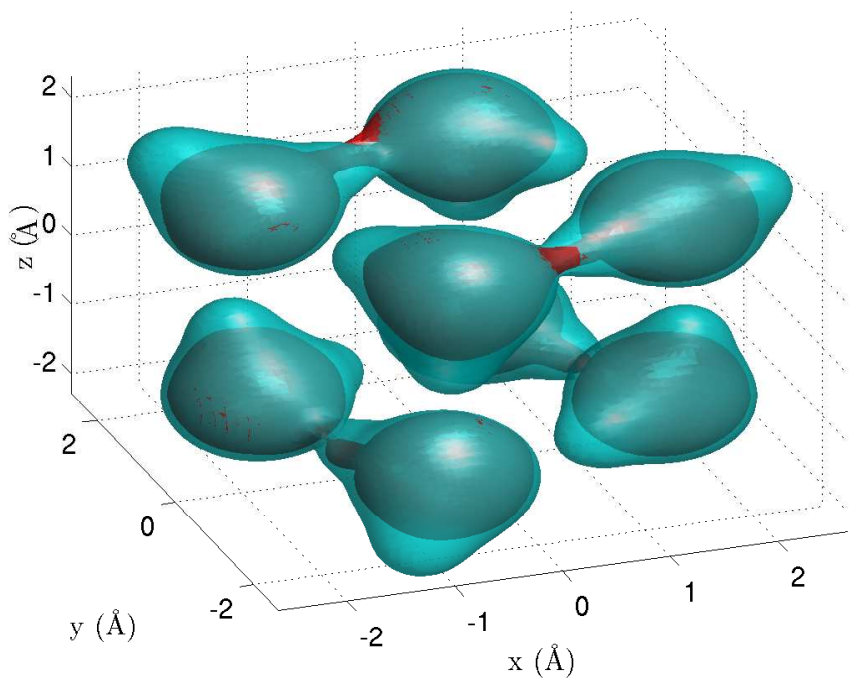

Figure 6.15. Electron density change during the Coulomb explosion in the field of $10^{16} \mathrm{~W} / \mathrm{cm}^{2}$. The isosurface value is $0.6 \AA^{-3}$, blue isosurface corresponds to the density at $0 \mathrm{fs}$, red to the density at $5 \mathrm{fs}$

The most important property of the sample for x-ray structural measurements is the electron density and its possible time-dependent change. Specific features of electron gas formation in a sample can affect x-ray scattering to a various degree. An advantage of using TDDFT from this point of view is that it offers ab-initio electron densities that follow the electron density changes in detail.

Figure 6.15 shows changes in electron density for the strongest field $\left(10^{16} \mathrm{~W} / \mathrm{cm}^{2}\right)$. The two isosurfaces correspond to the electron density of $0.6 \AA^{-3}$, with the blue isosurface corresponding to density at $\mathrm{t}=0 \mathrm{fs}$ and the inner red isosurface to density at time $\mathrm{t}=5 \mathrm{fs}$. It can be seen that with the electron density becoming more diffuse the isovalue moves closer to the positions of the ions. However, in order to evaluate the electron density changes in detail we have to switch from evaluation of the density in real space to Fourier space, which corresponds to scattering intensity spectra. 


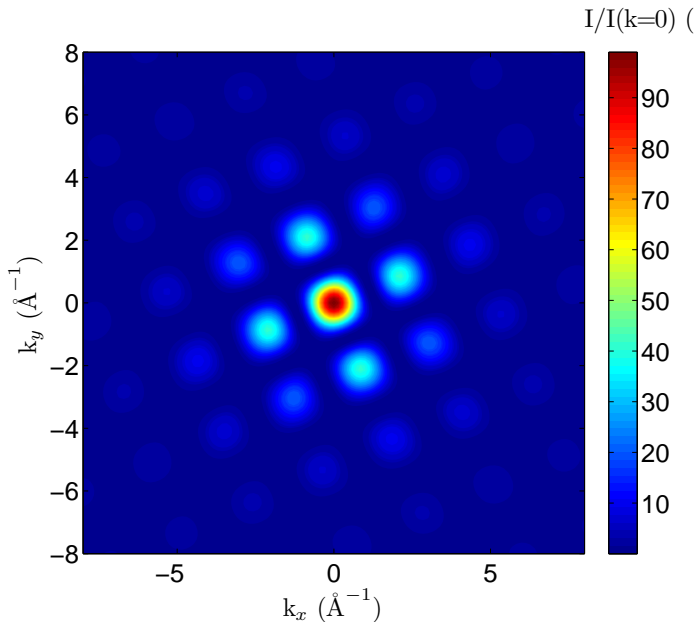

(a)

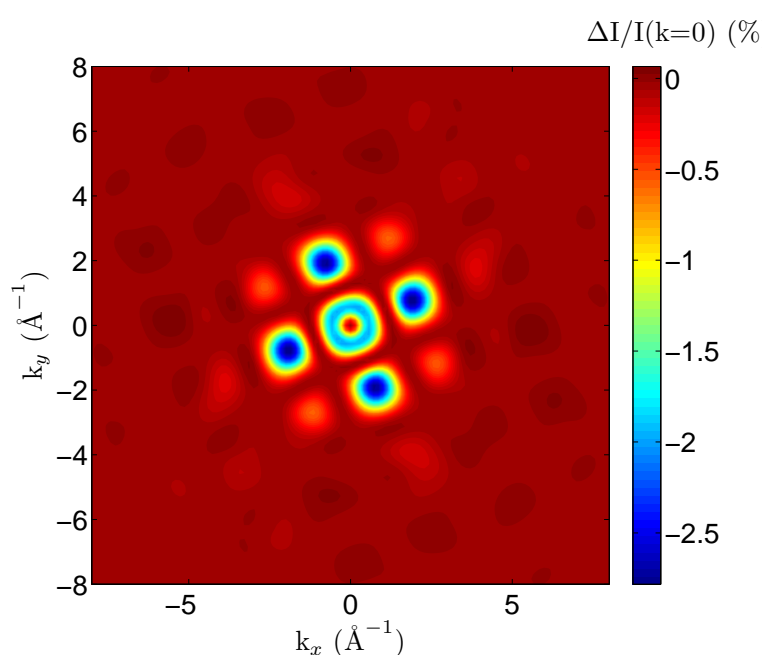

(b)

Figure 6.16. a) Scattering intensity of the water cluster at $t=0$ fs in $\%$ of the $k=0$ intensity and b) difference in scattering intensity between the intensity at $\mathrm{t}=0 \mathrm{fs}$ and $\mathrm{t}=$ $1 \mathrm{fs}$, under the laser field of $10^{16} \mathrm{~W} / \mathrm{cm}^{2}$

A simple way to evaluate the significance of the laser field caused structural damage for the structural measurements is the sensitivity of the x-ray scattering intensity to a change in the electron density $\delta \rho$. The scattering intensity can be 


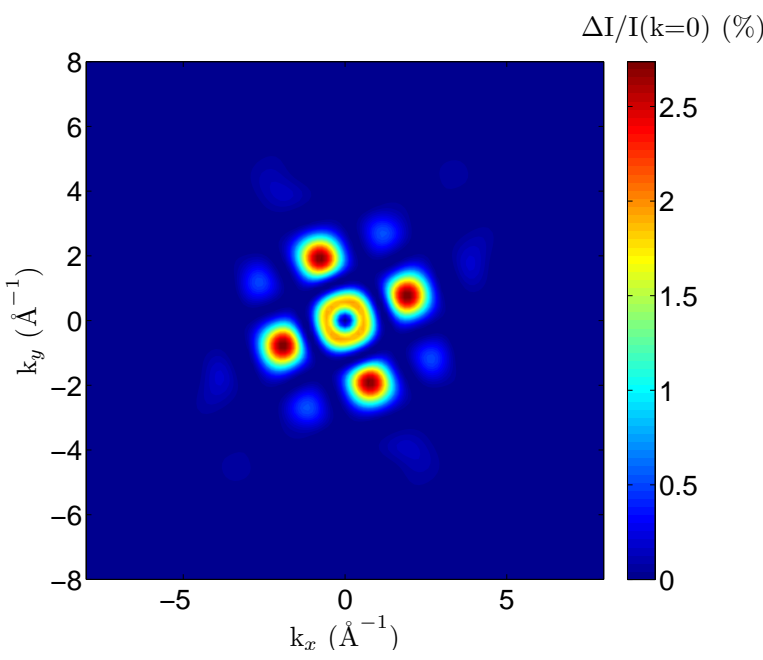

(a)

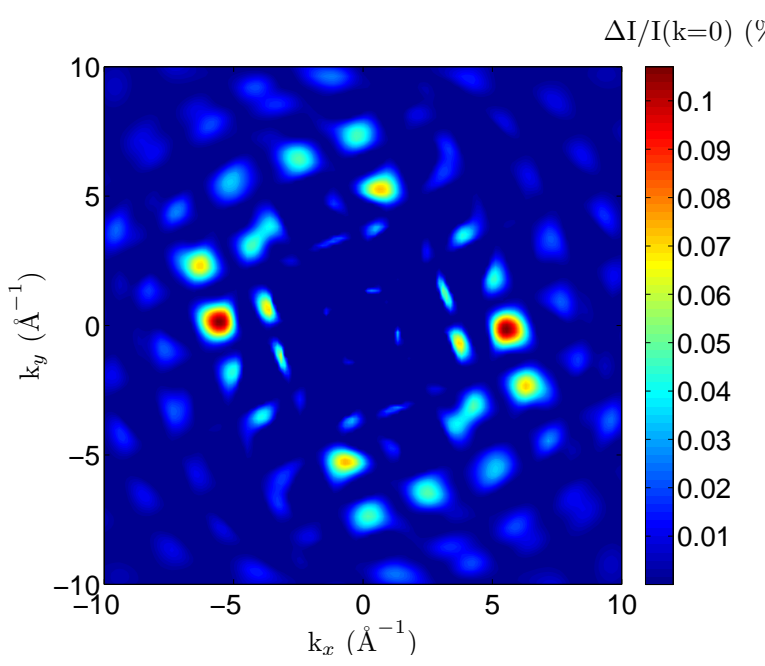

(b)

Figure 6.17. Difference in scattering intensity, in $\%$ of the $k=0$ intensity, between the intensity at $\mathrm{t}=0 \mathrm{fs}$ and $\mathrm{t}=1 \mathrm{fs}$ a) negative part and $\mathrm{b}$ ) positive part of the spectra under the laser field of $10^{16} \mathrm{~W} / \mathrm{cm}^{2}$

expressed simply as

$$
|F T(\rho+\delta \rho)|^{2}=|F T(\rho)+F T(\delta \rho)|^{2} .
$$

We have the time-dependent valence electron densities $\delta \rho(t)$ (hydrogen electrons 


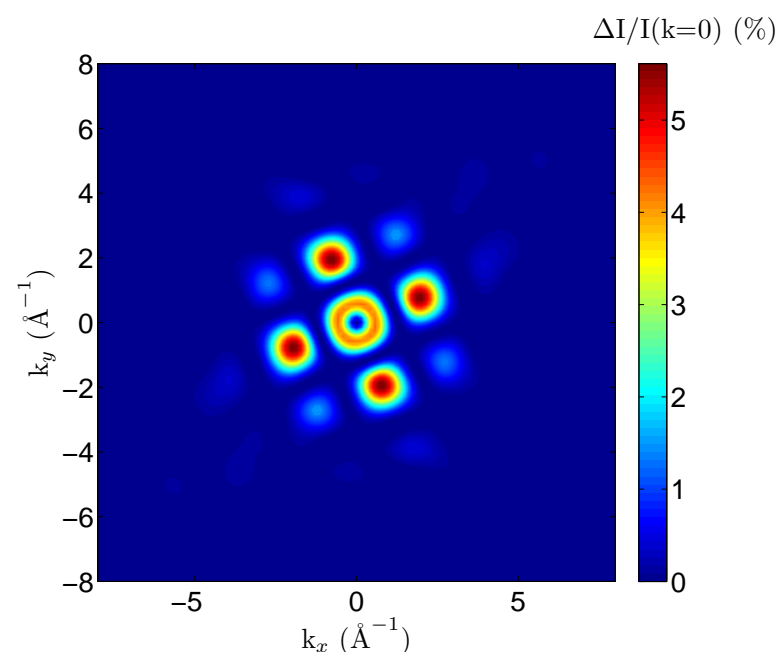

(a)

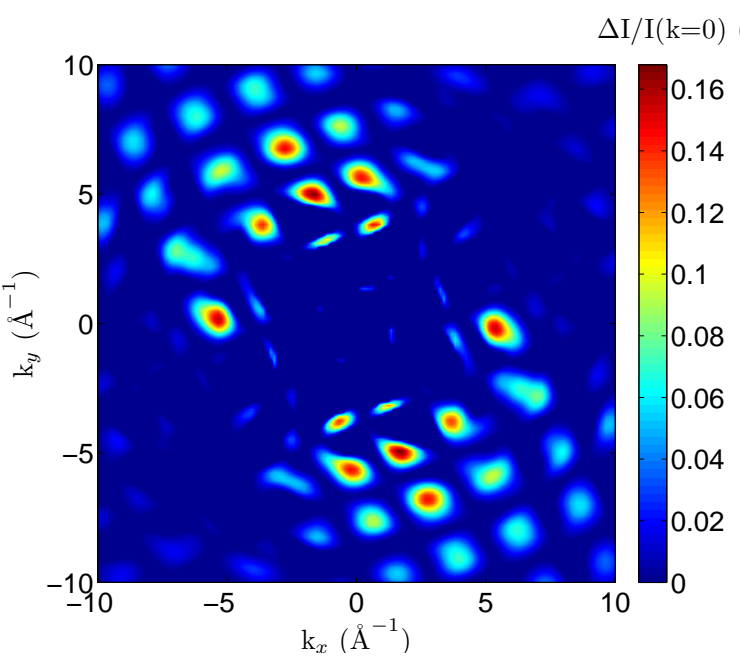

(b)

Figure 6.18. Difference in scattering intensity, in $\%$ of the $k=0$ intensity, between the intensity at $\mathrm{t}=0 \mathrm{fs}$ and $\mathrm{t}=2 \mathrm{fs}$; a) negative part of the spectra and $\mathrm{b}$ ) positive part of the spectra, under the laser field of $10^{16} \mathrm{~W} / \mathrm{cm}^{2}$

and the $2 s$ and $2 p$ electrons of oxygens) expressed on a spatial grid from the TDDFT calculations. Since these are smooth functions in comparison to the core electrons densities the Fourier transformation of these can be done using the fast Fourier transformation method (FFT). The 1s core electrons densities can be expressed using analytical functions, $\delta$ functions in the simplest approximation or 


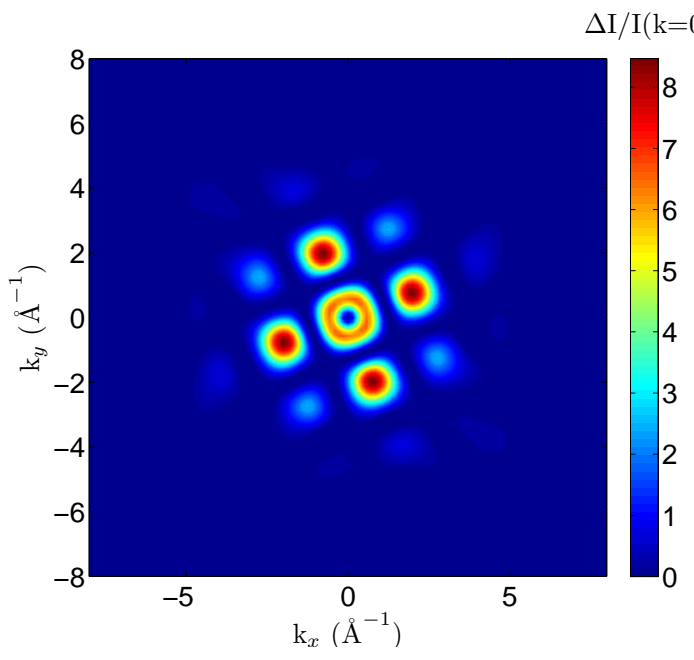

(a)

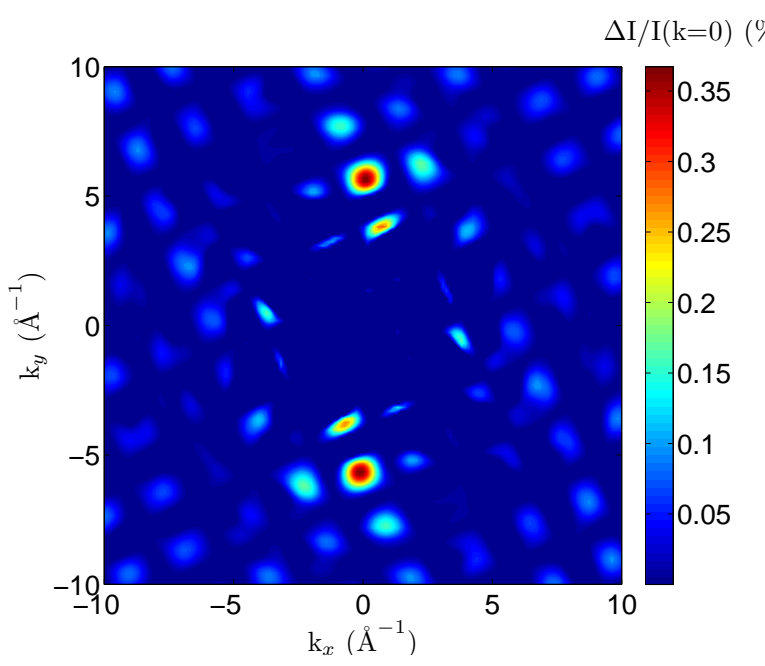

(b)

Figure 6.19. Difference in scattering intensity, in $\%$ of the $k=0$ intensity, between the intensity at $\mathrm{t}=0 \mathrm{fs}$ and $\mathrm{t}=3 \mathrm{fs}$; a) negative part of the spectra and $\mathrm{b}$ ) positive part of the spectra, under the laser field of $10^{16} \mathrm{~W} / \mathrm{cm}^{2}$

correctly chosen s-functions. In the simplest approach the $F T(\rho)$ of the core electrons will be assigned scattering factors and treated accordingly. This also separates the effects of the changes in valence electron density on the scattering spectra.

Figure 6.16 shows the scattering intensity of the water cluster at $t=0$ in $\%$ 


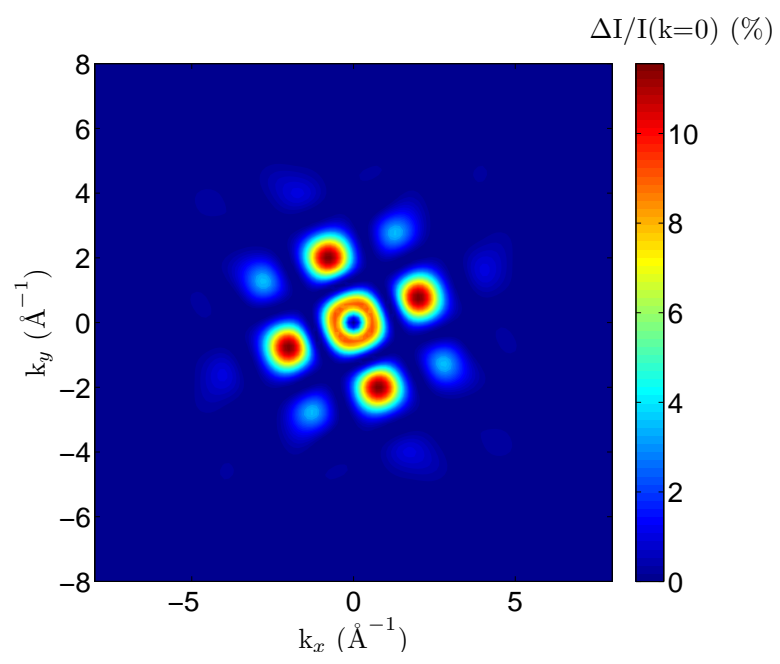

(a)

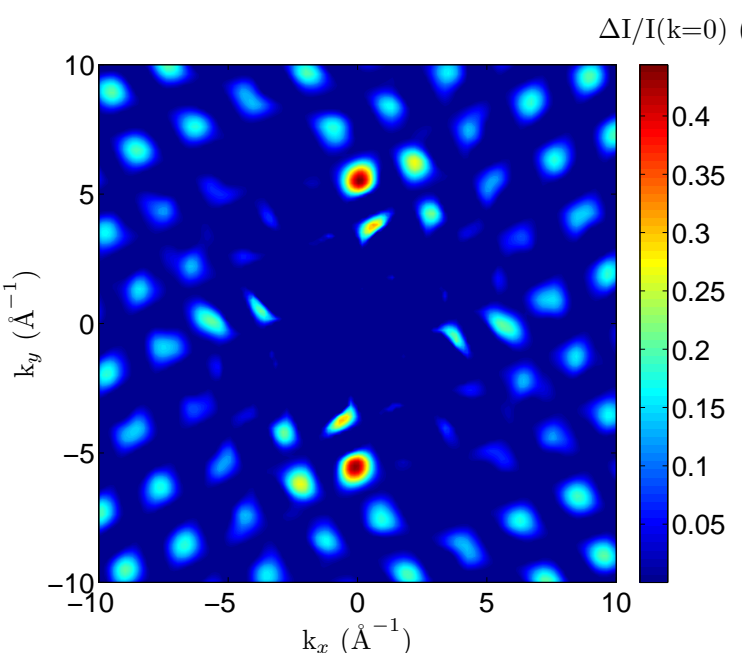

(b)

Figure 6.20. Difference in scattering intensity, in $\%$ of the $k=0$ intensity, between the intensity at $\mathrm{t}=0 \mathrm{fs}$ and $\mathrm{t}=4 \mathrm{fs}$; a) negative part of the spectra and $\mathrm{b}$ ) positive part of the spectra, under the laser field of $10^{16} \mathrm{~W} / \mathrm{cm}^{2}$

of the $I(k=0)$ intensity, with the scattering vector $\mathrm{k}=\frac{4 \pi \sin \theta}{\lambda}$, where $\theta$ is the scattering angle and $\lambda$ the $\mathrm{x}$-ray wavelength. The main maxima correspond to the oxygen atoms caused by the relatively higher number of electrons in comparison to the hydrogens. The hydrogens are included in the calculation in the electron density grid - they have no "core" electrons. This is an important fact to keep 


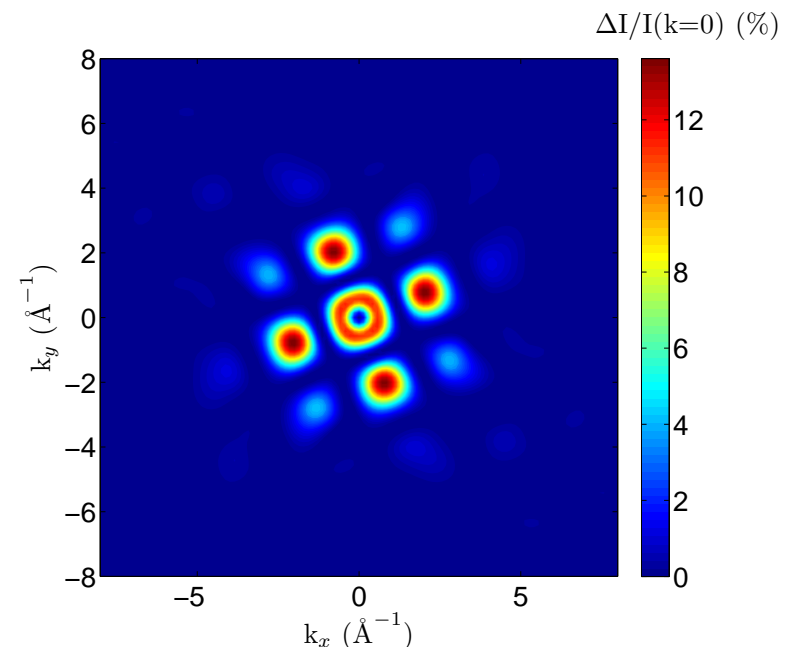

(a)

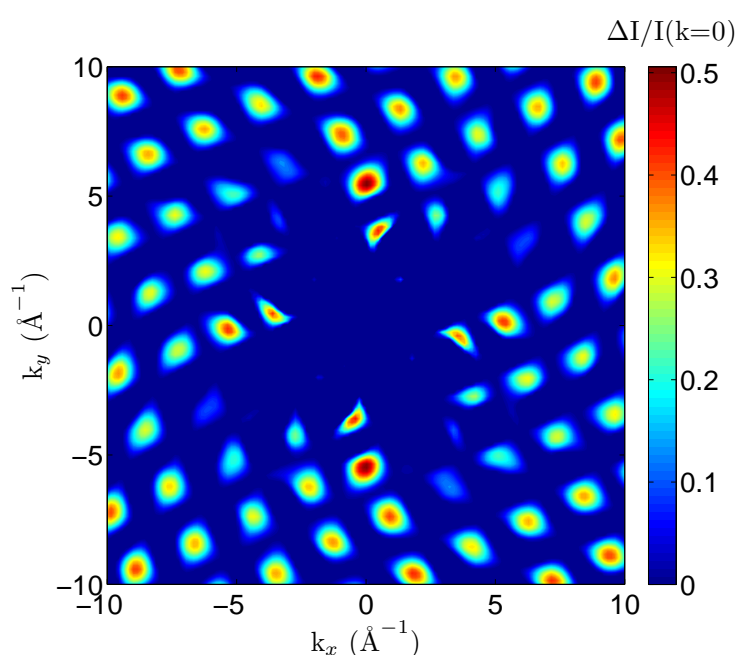

(b)

Figure 6.21. Difference in scattering intensity, in $\%$ of the $k=0$ intensity, between the intensity at $\mathrm{t}=0 \mathrm{fs}$ and $\mathrm{t}=5 \mathrm{fs}$; a) negative part of the spectra and $\mathrm{b}$ ) positive part of the spectra, under the laser field of $10^{16} \mathrm{~W} / \mathrm{cm}^{2}$

in mind while examining the time-dependent scattering intensity development.

The time dependent change of the intensity in the field of $10^{16} \mathrm{~W} / \mathrm{cm}^{2}$ is depicted in Figures 6.17 to 6.21 Here it can be seen that the four strong scattering maxima around $k=0$ are in a consistent fashion diminishing with time, while at the same time getting broader. The intensities from these maxima are redis- 


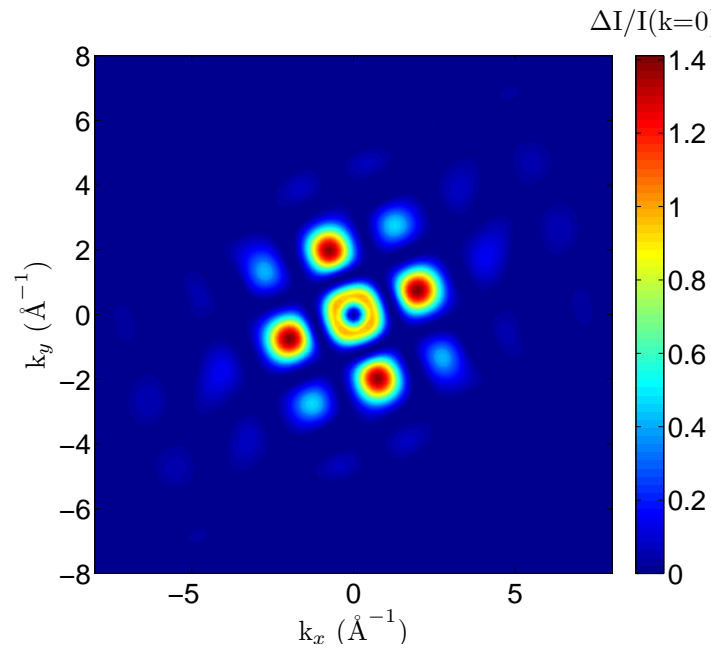

(a)

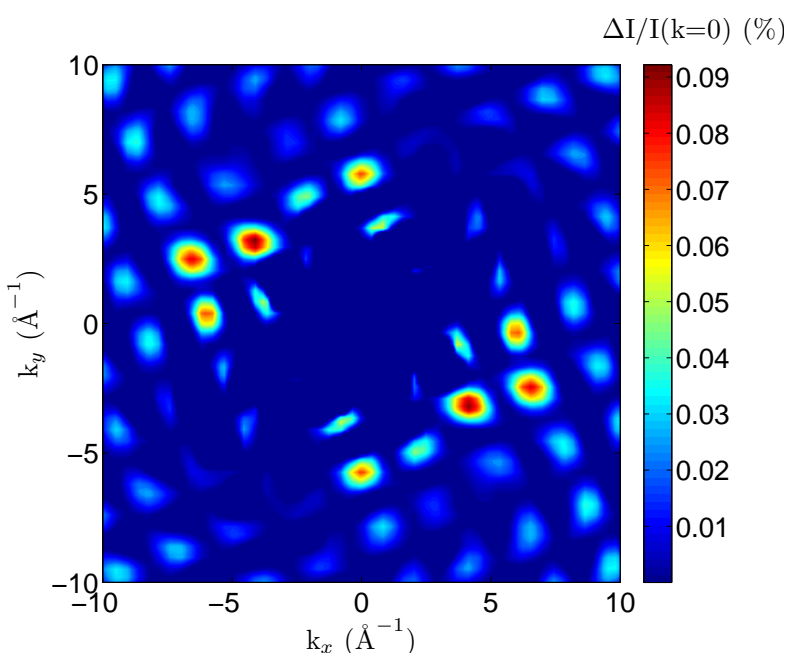

(b)

Figure 6.22. Difference in scattering intensity, in $\%$ of the $k=0$ intensity, between the intensity at $\mathrm{t}=0 \mathrm{fs}$ and $\mathrm{t}=5 \mathrm{fs}$; a) negative part of the spectra and $\mathrm{b}$ ) positive part of the spectra, under the laser field of $10^{15} \mathrm{~W} / \mathrm{cm}^{2}$

tributed to new features in the scattering map, especially in higher k-range. After $\mathrm{t}=5 \mathrm{fs}$ the scattering of this maxima decreases by about $13 \%$ of the $\mathrm{k}=0$ scattering intensity. This means that in the strongest field the scattering intensity becomes gradually smeared out, being redistributed from the strong oxygen maxima close to $\mathrm{k}=0$ to maxima at higher $\mathrm{k}$-range as the electrons start forming 
the quasi free electron gas. When focusing on the part of the scattering spectra that corresponds to the forming electron gas (Fig. 6.17b-6.21 b) these first form symmetrically around the main oxygen maxima. At later time points however, the electron gas part of the scattering spreads to larger scattering vector range. The shown intensity changes in the field of $10^{16} \mathrm{~W} / \mathrm{cm}^{2}$ are very strong, considering that the experimental $\mathrm{x}$-ray probe pulse is a number of times longer than the here studied $5 \mathrm{fs}$ time interval. This shows the very fast build up of the "inner ionized" electron gas, which degrades the scattering signal, in a sense degrading the scattering factors of the atoms in the sample.

Lastly, the negative and positive parts of scattering intensity after 5 fs in the weaker field of $10^{15} \mathrm{~W} / \mathrm{cm}^{2}$ are shown (Fig. 6.22). Although the atomic displacements in this field at this time point are comparable to the displacements in the strongest field at about $2 \mathrm{fs}$, the scattering intensity is only half as influenced, dropping by only about $1.4 \%$ instead of $2.5 \%$. From this example the necessity for the knowledge of the exact electron density, and not just the change in atomic positions, becomes obvious. 


\subsection{Conclusions}

Theoretical studies of x-ray laser-matter interaction have gained importance in recent years in connection with the proposed single molecule coherent diffraction measurements. These measurements are intended for relatively large molecular systems, especially various bio-molecules for which conventional crystallography is not an option. However, theoretical treatment of the interaction of large molecular systems with x-ray lasers necessarily employs severe approximations in order to decrease the computational costs. To describe the x-ray laser-matter interaction in a quantum mechanical ab-initio approach we have limited the size of the studied system to a small water cluster $\left(\mathrm{H}_{2} \mathrm{O}\right)_{8}$. In the current implementation in the OCTOPUS program package the external laser pulse field cannot treat hard x-ray energies, due to the dipole approximation and description of atomic core electrons by pseudopotentials. It is in principle possible to overcome these limitations and extend the method to the hard x-ray regime.

TDDFT may become an important tool in estimating what field strengths and pulse lengths are necessary to get a scattering signal of a needed quality. A detailed ab-initio analysis of the x-ray laser-matter interaction on small molecular systems can play an important role in choosing optimal field parameters for the structural measurements. We should however not forget the limitations the current density functionals impose. The time-dependent electron density measurement can provide a motivation for development of new density functionals, as an additional means of comparison of TDDFT performance with real electron wave function behavior.

In this work, one of the main advantages of the TDDFT approach is that the detailed ab-initio electron density of the system can be followed in time as it changes under the influence of the field. As shown in this Chapter the occupation of the molecular orbitals can be studied as a function of time. No assumptions have to be made about the nature of the excitation and ionization processes. This is particularly important for strong fields where nonlinear effects cannot be neglected, exemplified by the valence excitations evident in Figure 6.2 .

The strongest field considered in the simulation $\left(10^{16} \mathrm{~W} / \mathrm{cm}^{2}\right)$ leads to very fast cluster fragmentation, which renders a structural measurement after just a few femtoseconds meaningless. As it has been shown the scattering intensity on the main oxygen maxima drops by $12 \%$ in just 5 fs. The Coulomb explosion starts very 
fast with a electron plasma formation, the scattering signal of which contributes to the diffuse part of the scattering spectrum. Oxygen ions move relatively slowly even in the strongest field preserving the overall structure of the cluster.

Depending on the objectives of the experiment an encouraging result is the relatively well conserved overall symmetry and structure on short time scales. This is good news if one is interested in overall structure of the sample. However the possibility to determine any details within the structure will be lost very fast due to the fast plasma formation. As could be seen, although the actual ion movements during the first stages of the Coulomb explosion are minimal, especially for the heavier atoms, the scattering intensity is very sensitive to the electron plasma build-up, as shown in Section 6.5. Therefore it is important that simulations of Coulomb explosion accurately describe the detailed changes in electron density. 


\section{Chapter 7}

\section{Summary}

With the emergence of free electron x-ray laser sources, the field of x-ray structural measurements faces new challenges. The possibility to study the structure and dynamics of system with unparalleled resolution in time, owing to the ultrashort nature of the pulses, as well as to shed light on the structure of molecular systems that until now eluded us due to the problems with their crystallization. This thesis was written with the intention to contribute with a few small pieces to the puzzle that the field of x-ray scattering structure and dynamics studies has become. It was attempted to rationalize various physical parameters needed to be optimized in order to extract structural information from the ultrafast x-ray diffraction experiments of the present new and to be built x-ray sources.

First of all, x-ray sources with high brightness enhance the resolution of structural measurements simply by improving the signal to noise ratio, due to the increase in the photon count. This means enhancement for conventional scattering measurements. In order to study the possibilities of utilizing this effect we focus on the case of wide angle x-ray scattering measurements of liquid or gaseous samples. With the assumption of improved signal to noise ratio of the scattering measurement we conclude that the detailed electron densities become increasingly important as the diffuse part of the spectra, i.e. large scattering vectors, becomes measurable. Here, the small scale effects of the electron density, such as electron delocalization on bonds, or in phenyl rings in the studied case of stilbene, significantly contribute to the scattering signal. This to an extent where the use of standard scattering model, as in the Debye equation, would lead to a misin- 
terpretation of the spectra. In particular the Debye equation overestimates the sensitivity of wide angle x-ray scattering which can be misleading when interpreting experimental data.

X-ray scattering experiments bear significance even for processes that involve relatively small molecular systems. If for example one focuses on such a seemingly simple prototypical reaction as the photodissociation of molecular iodine a number of interesting effects can be brought forward. Relatively simple wave-packet dynamics of the molecular vibrations as it unfolds during the photodissociation gives rise to a strong time-dependent $x$-ray scattering signal change. Special interest here belongs to dynamical vibrational processes that can be triggered to unfold coherently in the studied sample, as this coherence gives a boost to the total scattering signal due to the constructive interference from the electron densities modulated by equivalent vibrational wave-packets of the nuclei. With considerable improvement to the x-ray scattering signal to noise ratio, to the extent when signal changes of $1 \%$ of the scattering intensity maximum at $\mathrm{k}=0$ would be distinguishable, even the smallest electron density change, as it occurs during vertical electronic excitation, can become measurable.

An interesting alternative to proposed single molecule coherent diffraction is x-ray scattering measurement on a sample consisting of aligned systems. Especially with the advances in the area of laser alignment this extension to x-ray scattering from amorphous liquid or gaseous samples shows a great potential. As we have shown, even the most simple one-dimensional alignment offers significant improvement compared to the structural information provided by a randomly oriented sample. Although it is sensitive to the uncertainty in the alignment angle, it offers encouraging results even at realistic alignment uncertainty distribution with $20^{\circ}$ full width at half maximum. Everywhere where a specific question about structural dynamics of a system is the main focus of an experiment, rather than high resolution structural analysis, one dimensional laser alignment may provide sufficient information about the structural change. Compared to the proposed single molecule coherent diffraction, laser alignment decreases the demands on the x-ray probe pulse brightness since it benefits from scattering intensity enhancement, due to constructive interference from the ensemble of aligned molecules. In this way the problems with Coulomb explosion do not even enter the picture.

With the interest in structural studies of single molecule or cluster structures 
comes the necessity to consider the dramatic event of Coulomb explosion. The effort has been not only to find the optimum for x-ray laser pulse length and intensity, but simultaneously to find an appropriate theoretical description for the laser-matter interaction itself. Time dependent density functional theory, in its form beyond linear response, shows promise when an ab-initio approach with an explicit treatment of the laser field is preferred, especially when dealing with smaller molecular systems. It is by definition well suited to provide the most important information from the x-ray scattering point of view, the detailed account of electron density changes in response to the strong laser field. This approach, although in its current implementation limited at the high energy end of the spectra to soft x-rays, has been used to study the Coulomb explosion of water octamer. The strongest field considered in the simulation $\left(10^{16} \mathrm{~W} / \mathrm{cm}^{2}\right)$ leads to very fast cluster fragmentation which would render a structural measurement after just few femtoseconds questionable. The Coulomb explosion starts very fast with an electron gas formation. It is first and foremost this electron gas formation that requires adequate theoretical description since it has significant influence on the x-ray scattering signal already in the first stages of the Coulomb explosion. When following the changes of electron density in Fourier space, in terms of scattering intensities, we have shown that the fast buildup of free electron gas in the cluster environment increasingly contributes to diffuse scattering signal. The main features of the cluster structure and symmetry stay well preserved due to the relatively slow moving ions.

Throughout this thesis only a small fraction of problems, that arise with the emergence of the free electron x-ray sources, have been addressed. Undoubtedly, a large number of additional challenges will spring up when the first experiments on one of these sources, namely the Linac Coherent Light Source in Stanford, begin in September 2009. 


\section{Bibliography}

1. Edman, K.; Nollert, P.; Royant, A.; Berlhall, H.; Pebay-Peyroula, E.; Hajdu, J.; Neutze, R.; M., L. E. Nature 1999, 401, 822.

2. Techert, S.; Schotte, F.; Wulff, M. Phys. Rev. Lett 2001, 86, 2030.

3. Siders, C. W.; Cavalleri, A.; Sokolowski-Tinten, K.; Tóth, C.; Guo, T.; Kammler, M.; Horn von Hoegen, M.; Wilson, K. R.; von der Linde, D.; Barty, C. P. J. Science 1999, 286, 1340.

4. Henderson, R. Proc. R. Soc. Lond. B 1990, 241, 6.

5. Neutze, R.; Wouts, R.; van der Spoel, D.; Weckert, E.; Hajdu, J. Nature 2000, 406, 752 .

6. Miao, J.; Charalambous, P.; Kirz, J.; Sayre, D. Nature 2006, 400, 342.

7. Chapman, H. N.; Barty, A.; Marchesini, S.; Noy, A.; Hau-Riege, S. P.; Cui, C.; Howells, M. R.; Rosen, R.; He, H.; Spence, J. C. H.; Weierstall, U.; Beetz, T.; Jacobsen, C.; Shapiro, D. J. Opt. Soc. Am. A 2009, 23, 1179.

8. Barty, A.; Boutet, S.; Bogan, M. J.; Hau-Riege, S.; Marchesini, S.; Sokolowski-Tinten, K.; Stojanovic, N.; Tobey, R.; Ehrke, H.; Cavalleri, A.; Düsterer, S.; Frank, M.; Bajt, S.; Woods, B.; Siebert, M. M.; Hajdu, J.; Treusch, R. Nature Photon. 2008, 2, 415.

9. Jenssen, F. Introduction to Computational Chemistry; John Wiley \& Sons Ltd., 1999.

10. Domcke, W., Yarkony, D. R., Köppel, H., Eds. Conical Intersections Electronic Structure Dynamics and Spectroscopy; World Scientific, 2004. 
11. May, V.; Kühn, O. Charge and Energy Transfer Dynamics in Molecular Systems; WILEY-VCH Verlag, 2000.

12. Ditchfield, R.; Hehre, W. J.; Pople, J. A. J. Chem. Phys. 1971, 54, 724.

13. Hehre, W. J.; Ditchfield, R.; Pople, J. A. J. Chem. Phys. 1972, 56, 2257.

14. Helliwell, J. R., Rentzepis, P. M., Eds. Time-resolved Diffraction; Oxford University Press, 1997.

15. Ben-Num, M.; Martínez, T. J.; Weber, P. M.; Wilson, K. R. Chem. Phys. Lett. 1996, 262, 405.

16. Ben-Num, M.; Cao, J.; Wilson, K. R. J. Phys. Chem. A 1997, 101, 8743.

17. Cao, J.; Wilson, K. R. J. Phys. Chem. A 1998, 102, 9523.

18. Parr, R. G.; Yang, W. Density Functional Theory of Atoms and Molecules; Oxford University Press, 1994.

19. Gross, E. K. U.; Rubio, A.; Burke, K.; van Leeuwen, R.; Marques, M. A. L.; de Boeij, P.; Marini, A.; Stefanucci, G.; Dahlen, N. E.; Almbladh, C.-O. Time-Dependent Density Functional Theory; Springer Berlin / Heidelberg, 2006.

20. Fiolhais, C., Nogueira, F., Marques, M., Eds. Primer in Density Functional Theory; Springer Verlag, 2003.

21. Thomas, L. H. Proc. Cambridge Phil. Soc. 1927, 23, 542.

22. Teller, E. Rev. Mod. Phys. 2001, 34, 627.

23. Lee, C.; Yang, W.; Parr, R. G. Phys. Rev. B 1988, 37, 785.

24. Becke, A. D. Phys. Rev. B 1988, 38, 3098.

25. Perdew, J. P.; Wang, Y. Phys. Rev. B 1986, 33, 8800.

26. Runge, E.; Gross, E. K. U. Phys. Rev. Lett 1984, 52, 997.

27. Casida, M. E. Recent Developments and Application of Modern Density Functional Theory 1996, page 391. 
28. Castro, A.; Appel, H.; Oliveira, M.; Rozzi, A.; Andrade, X.; Lorenzen, F.; Marques, M. A. L.; Gross, E. K. U.; Rubio, A. Phys. Stat. Sol. (b) 2006, 243, 2465.

29. Verlet, L. Phys. Rev. 1967, 159(1), 98.

30. Verlet, L. Phys. Rev. 1968, 165, 201.

31. Beeman, D. J. Comput. Phys. 1976, 20, 130.

32. Allen, M. P.; Tildesley, D. J. Computer Simulation of Liquids; Oxford University Press: Oxford, U.K., 1987.

33. Billing, G. D.; Mikkelsen, K. V. Advanced Molecular Dynamics and Chemical Kinetics; John Wiley \& Sons, Inc., 1997.

34. Kosloff, R. Ann. Rev. Chem. 1994, 45, 145.

35. Cullum, J. K.; Willoughby, R. A. Lanczos Algorithms for Large Symmetric Eigenvalue Computations; Birkhäuser: Boston, 1985.

36. Neutze, R.; Wouts, R.; Techert, S.; Davidsson, J.and Kocsis, M.; Kirrander, A.; Schotte, F.; Wulff, M. Phys. Rev. Lett 2001, 87, 195508.

37. Davidsson, J.; Poulsen, J.; Cammarata, M.; Georgiou, P.; Wouts, R.; Katona, G.; Jacobson, F.; Plech, A.; Wulff, M.; Nyman, G.; Neutze, R. Phys. Rev. Lett 2005, 94(24), 245503.

38. Ihee, H.; Lorenc, M.; Kim, T. K.; Kong, Q. Y.; Cammarata, M.; Lee, J. H.; Bratos, S.; Wulff, M. Science 2005, 309(5738), 1223.

39. Lindenberg, A. M.; Acremann, Y.; Lowney, D. P.; Heimann, P. A.; Allison, T. K.; Matthews, T.; Falcone, R. W. J. Chem. Phys. 2005, 122(20), 204507.

40. Techert, S.; Schmatz, S. Z. Phys. Chem. 2002, 216, 575.

41. Bressler, C.; Chergui, M. Chem. Rev. 2004, 104, 1781.

42. Srajer, V.; Teng, T.-Y.; Ursby, T.; Pradevard, C.; Ren, Z.; Adachi, S.; Schildkamp, W.; Bourgeois, D.; Wulff, M.; Moffat, K. Science 1996, 274, 1726. 
43. Rousse, A.; Rischel, C.; Fourmaux, S.; Uschmann, I.; Sebban, S.; Grillon, G.; Balcou, P.; Föster, E.; Geindre, J. P.; Audebert, P.; Gauthier, J. C.; Hulin, D. Nature 2001, 410(6824), 65.

44. Sokolowski-Tinten, K.; Blome, C.; Blums, J.; Cavalleri, A.; Dietrich, C.; Tarasevitch, A.; Uschmann, I.; Förster, E.; Kammler, M.; von Hoegen, M. H.; von der Linde, D. Nature 2003, 422(6926), 287.

45. Lee, T.; Jiang, Y.; Rose-Petruck, C. G.; Benesch, F. J. Chem. Phys. 2005, $122(8), 084506$.

46. Rose-Petruck, C.; Jimenez, R.; Guo, T.; Cavalleri, A.; Siders, C. W.; Raksi, F.; Squier, J. A.; Walker, B. C.; Wilson, K. R.; Barty, C. P. J. Nature 1999, 398,310 .

47. Cavalieri, A. L.; Fritz, D. M.; Lee, S. H.; Bucksbaum, P. H.; Reis, D. A.; Rudati, J.; Mills, D. M.; Fuoss, P. H.; Stephenson, G. B.; Kao, C. C.; Siddons, D. P.; Lowney, D. P.; MacPhee, A. G.; Weinstein, D.; Falcone, R. W.; Pahl, R.; Als-Nielsen, J.; Blome, C.; DÃ $\frac{1}{4}$ sterer, S.; Ischebeck, R.; Schlarb, H.; Schulte-Schrepping, H.; Tschentscher, T.; Schneider, J.; Hignette, O.; Sette, F.; Sokolowski-Tinten, K.; Chapman, H. N.; Lee, R. W.; Hansen, T. N.; Synnergren, O.; Larsson, J.; Techert, S.; Sheppard, J.; Wark, J. S.; Bergh, M.; Caleman, C.; Huldt, G.; van der Spoel, D.; Timneanu, N.; Hajdu, J.; Akre, R. A.; Bong, E.; Emma, P.; Krejcik, P.; Arthur, J.; Brennan, S.; Gaffney, K. J.; Lindenberg, A. M.; Luening, K.; Hastings, J. B. Phys. Rev. Lett 2005, 94(11), 114801.

48. Arthur, J.; others. Lcls conceptual design report Technical report, SLAC-R593 LCLS, Menlo Park, CA, USA, 2002.

49. Materlik, G.; Tschentscher, T. Eu-xfel - technical design report Technical report, Hasylab/DESY, 2006.

50. Bilderback, D. H.; Elleaume, P.; Weckert, E. J. Phys. B 2005, 38, 773.

51. Koppel, H.; Domcke, W.; Cederbaum, L. S. Adv. Chem. Phys. 1984, 57, 59.

52. Krause, J. L.; Whitnell, R.; Wilson, K. R.; Yan, Y. J. J. Chem. Phys. 1993, 99,6562 . 
53. Kohler, B.; Yakovlev, V. V.; Che, J.; Krause, J. L.; Messina, M.; Wilson, K. R. Phys. Rev. Lett 1995, 74, 3360.

54. Feil, D. J. Mol. Struct.: THEOCHEM 1992, 255, 221.

55. Tsirelson, V. G.; Ozerov, R. P. J. Mol. Struct.: THEOCHEM 1992, 87, 335.

56. Coppens, P. X-ray charge densities and chemical bonding.; Oxford University Press: Oxford, 1997.

57. Boys, S. F. Proc. R. Soc. London A 1960, 258(1294), 402.

58. Hohenberg, P.; Kohn, W. Phys. Rev. B 1964, 136(3B), 864.

59. Feil, D. Isr. J. Chem. 1977, 16, 103.

60. Warren, B. E. X-Ray Diffraction; Addison-Wesley: Reading, MA, 1969.

61. Syage, J. A.; Felker, P. M.; Zewail, A. H. J. Chem. Phys. 1984, 81(11), 4685.

62. Ishii, K.; Takeuchi, S.; Tahara, T. Chem. Phys. Lett. 2004, 398(4-6), 400.

63. Schroeder, J.; Troe, J. Activated Barrier Crossing; G. R. Fleming and P. Haenggi, World Scientific Press: Singapore, 1993.

64. Syage, J. A.; Lambert, W.; Felker, P. M.; Zewail, A. H.; Hochstrasser, R. Chem. Phys. Lett. 1982, 88, 266.

65. Troe, J.; Weitzel, K.-M. J. Chem. Phys. 1988, 88, 7030.

66. Balk, M. W.; Fleming, G. R. J. Phys. Chem. 1986, 90, 3975.

67. Schroeder, J.; Schwarzer, D.; Troe, J.; Voss, F. J. Chem. Phys. 1990, 93, 2393.

68. Mohrschladt, R.; Schroeder, J.; Troe, J.; Vöhringer, P. J. Chem. Phys. 1994, 101,7566 .

69. Dietl, C.; Papastathopoulos, E.; Niklaus, P.; Improta, R.; Santoro, F.; Gerber, G. Chem. Phys. 2005, 310(1-3), 201.

70. Improta, R.; Santoro, F. J. Phys. Chem. A 2005, 109(44), 10058. 
71. Gagliardi, L.; Orlandi, G.; Molina, V.; Malqvist, P.-Å.; Roos, B. J. Phys. Chem. A 2002, 106(32), 7355.

72. Gaussian 03, Revision C.02. Frisch, M. J.; Trucks, G. W.; Schlegel, H. B.; Scuseria, G. E.; Robb, M. A.; Cheeseman, J. R.; Montgomery, Jr., J. A.; Vreven, T.; Kudin, K. N.; Burant, J. C.; Millam, J. M.; Iyengar, S. S.; Tomasi, J.; Barone, V.; Mennucci, B.; Cossi, M.; Scalmani, G.; Rega, N.; Petersson, G. A.; Nakatsuji, H.; Hada, M.; Ehara, M.; Toyota, K.; Fukuda, R.; Hasegawa, J.; Ishida, M.; Nakajima, T.; Honda, Y.; Kitao, O.; Nakai, H.; Klene, M.; Li, X.; Knox, J. E.; Hratchian, H. P.; Cross, J. B.; Bakken, V.; Adamo, C.; Jaramillo, J.; Gomperts, R.; Stratmann, R. E.; Yazyev, O.; Austin, A. J.; Cammi, R.; Pomelli, C.; Ochterski, J. W.; Ayala, P. Y.; Morokuma, K.; Voth, G. A.; Salvador, P.; Dannenberg, J. J.; Zakrzewski, V. G.; Dapprich, S.; Daniels, A. D.; Strain, M. C.; Farkas, O.; Malick, D. K.; Rabuck, A. D.; Raghavachari, K.; Foresman, J. B.; Ortiz, J. V.; Cui, Q.; Baboul, A. G.; Clifford, S.; Cioslowski, J.; Stefanov, B. B.; Liu, G.; Liashenko, A.; Piskorz, P.; Komaromi, I.; Martin, R. L.; Fox, D. J.; Keith, T.; Al-Laham, M. A.; Peng, C. Y.; Nanayakkara, A.; Challacombe, M.; Gill, P. M. W.; Johnson, B.; Chen, W.; Wong, M. W.; Gonzalez, C.; Pople, J. A.

73. Becke, A. J. Chem. Phys. 1996, 104, 1040.

74. Pedrew, J. D. Phys. Rev. B 1986, 33, 8822.

75. Wiberg, K. B.; de Oliveira, A. E.; Trucks, G. J. Phys. Chem. A 2002, 106(16), 4192 .

76. Felker, P. M.; Zewail, A. H. J. Phys. Chem. A 1985, 89(25), 5402.

77. Leitner, D. M.; Levine, B.; Quenneville, J.; Martínez, T. J.; Wolynes, P. G. J. Phys. Chem. A 2003, 107(49), 10706.

78. Gaussview, Version 3.09. Dennington II, R.; Keith, T.; Millam, J.; Eppinnett, K.; Hovell, W. L.; Gilliland, R.

79. Feit, M. D.; Fleck Jr., J. A.; Steiger, A. Jour. Comp. Phys. 1982, 47, 412.

80. T. J. Chuang, G. W. H.; Eisenthal, K. B. Chem. Phys. Lett. 1974, 25, 201.

81. D. F. Kelley, N. A. A.-H.; Jang, D. J. Chem. Phys. 1984, 80, 4105. 
82. A. L. Harris, M. B.; Harris, C. B. J. Chem. Phys. 1986, 84, 788.

83. Abul-Haj, N. A.; Kelley, D. F. J. Chem. Phys. 1986, 84, 1335.

84. Harris, A. L.; Brown, J. K.; Harris, C. B. Ann. Rev. Phys. Chem. 1988, 39, 341.

85. Bratos, S.; Mirloup, F.; Vuilleumier, R. J. Chem. Phys. 2002, 116, 10615.

86. Plech, A.; Wulff, M.; Bratos, S.; Mirloup, F.; Vuilleumeir, R.; Schotte, F.; Anfinrud, A. Phys. Rev. Lett 2001, 92, 125505.

87. Wulff, M.; Bratos, S.; Plech, A.; Vuilleumeir, R.; Mirloup, F.; Lorenc, M.; Kong, Q.; Ihee, H. J. Chem. Phys. 2006, 124, 034501.

88. Kong, Q.; Wulff, M.; Bratos, S.; Vuilleumeir, R.; Kim, J.; Ihee, H. J. Phys. Chem. 2006, 110, 11178.

89. Smith, D. E.; Harris, C. B. J. Chem. Phys. 1987, 87, 2709.

90. Rabinovitch, E.; Wood, C. W. Trans. Faraday. Soc. 1936, 32, 547.

91. P. Bado, C. Dupuy, D. M.; Wilson, K. R. J. Chem. Phys. 1984, 80, 5531.

92. M. Berg, A. L. H.; Harris, C. B. Phys. Rev. Lett 1985, 54, 951.

93. Mulliken, R. S. J. Chem. Phys. 1971, 55, 288.

94. Das, G.; Wahl, A. C. J. Chem. Phys. 1978, 69, 53.

95. Teichteil, C.; Pelissier, M. Chem. Phys 1994, 180, 1.

96. Dolg, M. Mol. Phys. 1996, 88, 1645.

97. Viguè, J.; Broyer, M.; Lehman, J. C. J. Chem. Phys. 1981, 42, 949.

98. Viguè, J.; Broyer, M.; Lehman, J. C. J. Chem. Phys. 1981, 42, 961.

99. Viguè, J.; Broyer, M.; Lehman, J. C. J. Chem. Phys. 1981, 42, 937.

100. de Jong, W. A.; Vischer, L.; Nieuwpoort, W. C. J. Chem. Phys. 1997, 107, 9046.

101. Ho, P. J.; Santra, R. Phys. Rev. A 2008, 78, 053409. 
102. Larsen, J. J.; Sakai, H.; Safvan, C. P.; Wendt-Larsen, I.; Stapelfelds, H. J. Chem. Phys. 1999, 111, 7774.

103. Viftrup, S. S.; Kumarappan, V.; Trippel, S.; Stapelfeldt, H. Phys. Rev. Lett 2007, 99, 143602.

104. Tehini, R.; Sugny, D. Phys. Rev. A 2008, 77, 023407.

105. Pelzer, A.; Ramakrishna, S.; Seideman, T. J. Chem. Phys. 2008, 129, 134301.

106. Stapelfeldt, H.; Seideman, T. Rev. Mod. Phys. 2003, 75, 543.

107. Zholents, A. A.; Zolotorev, M. S. New Journal of Physics 2008, 10, 025005.

108. Saldin, E. L.; Schneidmiller, E. A.; Yurkov, M. V. Physical Review Special Topics - Accelerators and Beams 2006, 9, 050702.

109. Stapelfeldt, H. Eur. Phys. J. D 2003, 26, 15.

110. Saalmann, U.; Siedschlag, C.; Rost, J. M. J. Phys. B: At. Mol. Opt. Phys. 2006, 39, R39.

111. Yamanouchi, K. Science 2002, 295, 1695.

112. Miao, J.; Chapman, H. N.; Kirz, J.; Sayre, D.; Hodgson, K. O. Annu. Rev. Biophys. Biomol. Struct. 2004, 33, 157.

113. Gaffney, K. J.; Chapman, H. N. Science 2007, 316, 1444.

114. Chapman, H. N. Nature Mater. 2009, 8, 29.

115. Hau-Riege, S. P.; London, R. A.; Huldt, G.; Chapman, H. N. Phys. Rev. E 2005, 71, 061919 .

116. Hau-Riege, S. P.; London, R. A.; Szöke, A. Phys. Rev. E 2004, 69, 051906.

117. Jurek, Z.; Faigel, G.; Tegze, M. Eur. Phys. J. D 2004, 29, 217.

118. Jurek, Z.; Faigel, G. Europhys. Lett. 2004, 65, 491.

119. Bergh, M.; Tîmneanu, N.; van der Spoel, D. Phys. Rev. E 2004, 70, 051904.

120. Hau-Riege, S. P.; London, R. A.; Chapman, H. N.; Bergh, M. Phys. Rev. E 2007, 76, 046403. 
121. Karthikeyan, S.; Park, M.; Shin, I.; Kim, K. S. J. Phys. Chem. A 2008, 112, 10120.

122. Gruenloh, C. J.; Carney, J. R.; Arrington, C. A.; Zwier, T. S.; Fredericks, S. Y.; Jordan, K. D. Science 1997, 276, 1678.

123. Du, Q.; Superfine, R.; Freysz, E.; Shen, Y. Phys. Rev. Lett 1993, 70, 2313.

124. Rowland, B.; Kadagathur, N.; Devlin, J.; Buch, V.; Feldman, T.; Wojcik, M. J. Chem. Phys. 1995, 102, 8328.

125. Marques, M.; Castro, A.; Bertsch, G. F.; Rubio, A. Comput. Phys. Comm. 2002, 151, 60 .

126. Isla, M.; Alonso, J. A. Phys. Rev. A 2005, 72, 023201.

127. Livshits, E.; Baer, R. J. Phys. Chem. 2006, 110, 8443.

128. Baer, R.; Neuhauser, D. Phys. Rev. Lett 2005, 94, 043002.

129. Ziegler, T.; Seth, M.; Krykunov, M.; Autschbach, J. J. Chem. Phys. 2008, $129,184114$.

130. Yang, B. X.; J., K. Phys. Rev. B 1987, 36, 1361.

131. Hayashi, H.; Watanabe, N.; Udagawa, Y.; Kao, C. PNAS 2000, 97, 6264.

132. Veigele, W. J. Atomic Data 1973, 5, 51.

133. Timneanu, N.; Caleman, C.; Hajdu, J.; van der Spoel, D. Chem. Phys. 2004, $299,277$.

134. The Cambridge Cluster Database. Wales, D. J.; Doye, J. P. K.; Dullweber, A.; Hodges, M. P.; Naumkin, F. Y.; Calvo, F.; Hernández-Rojas, J.; Middleton, T. F. http://www-wales.ch.cam.ac.uk/CCD.html. 


\section{Appendix A}

\section{Program for x-ray scattering on aligned molecules}

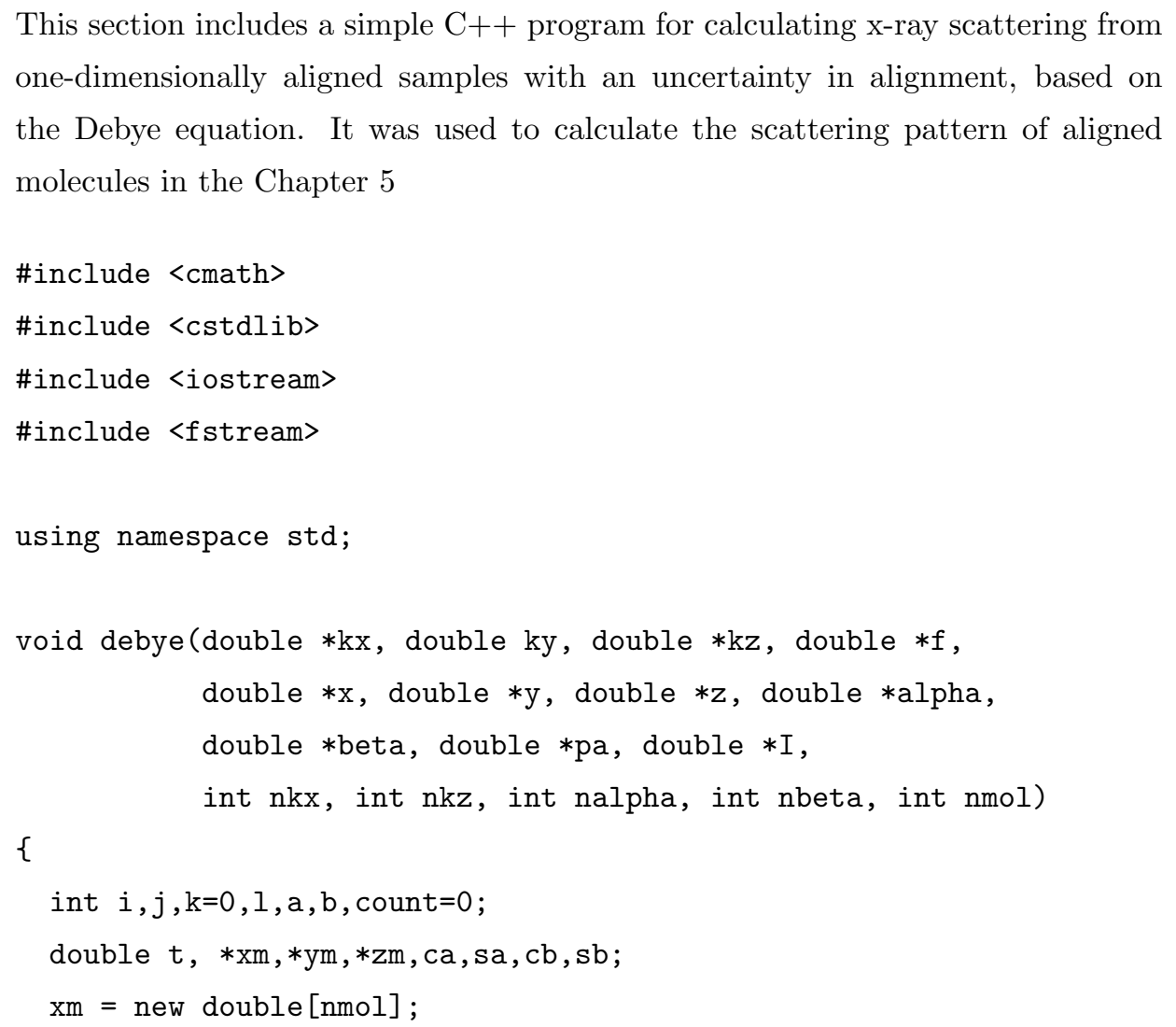




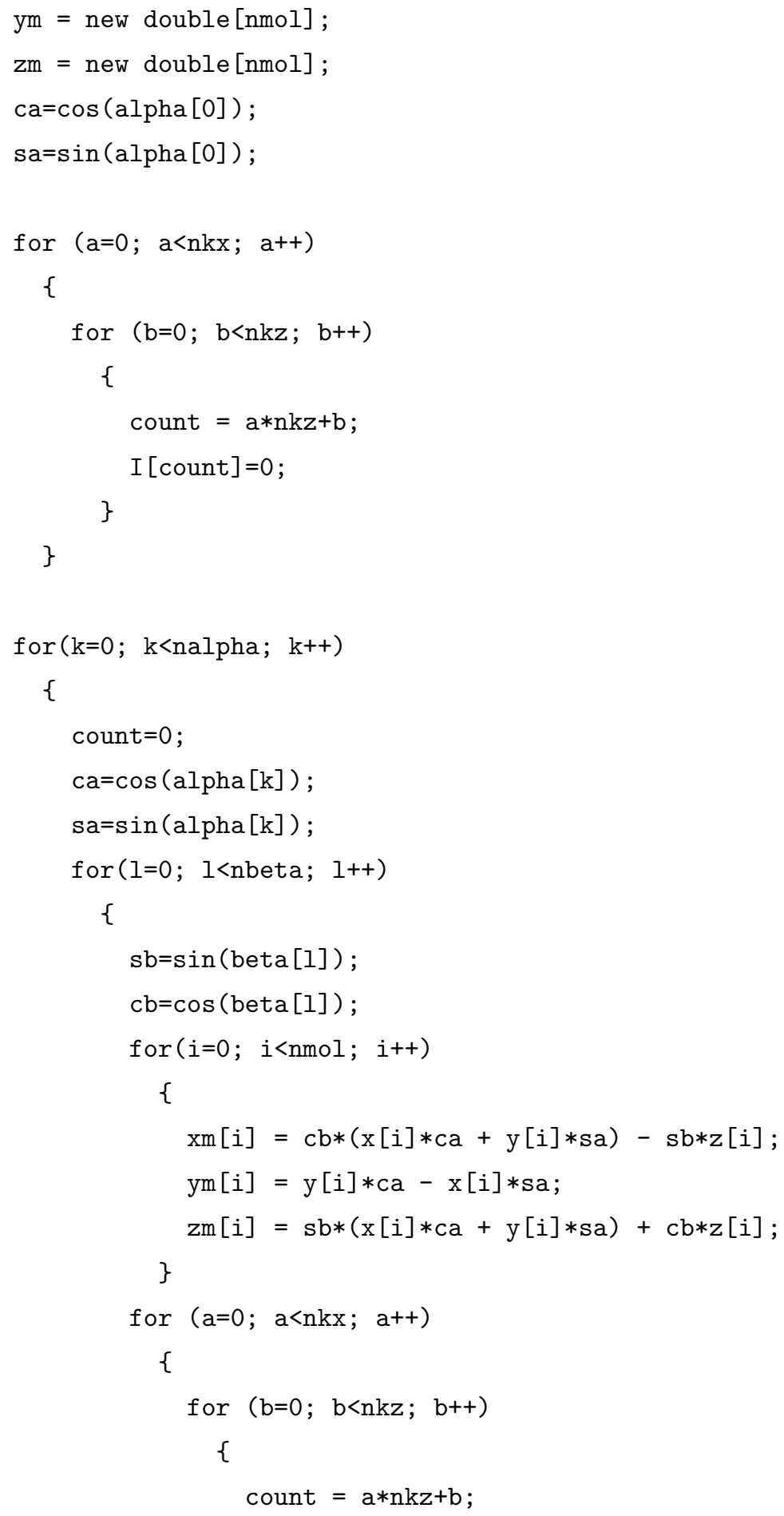




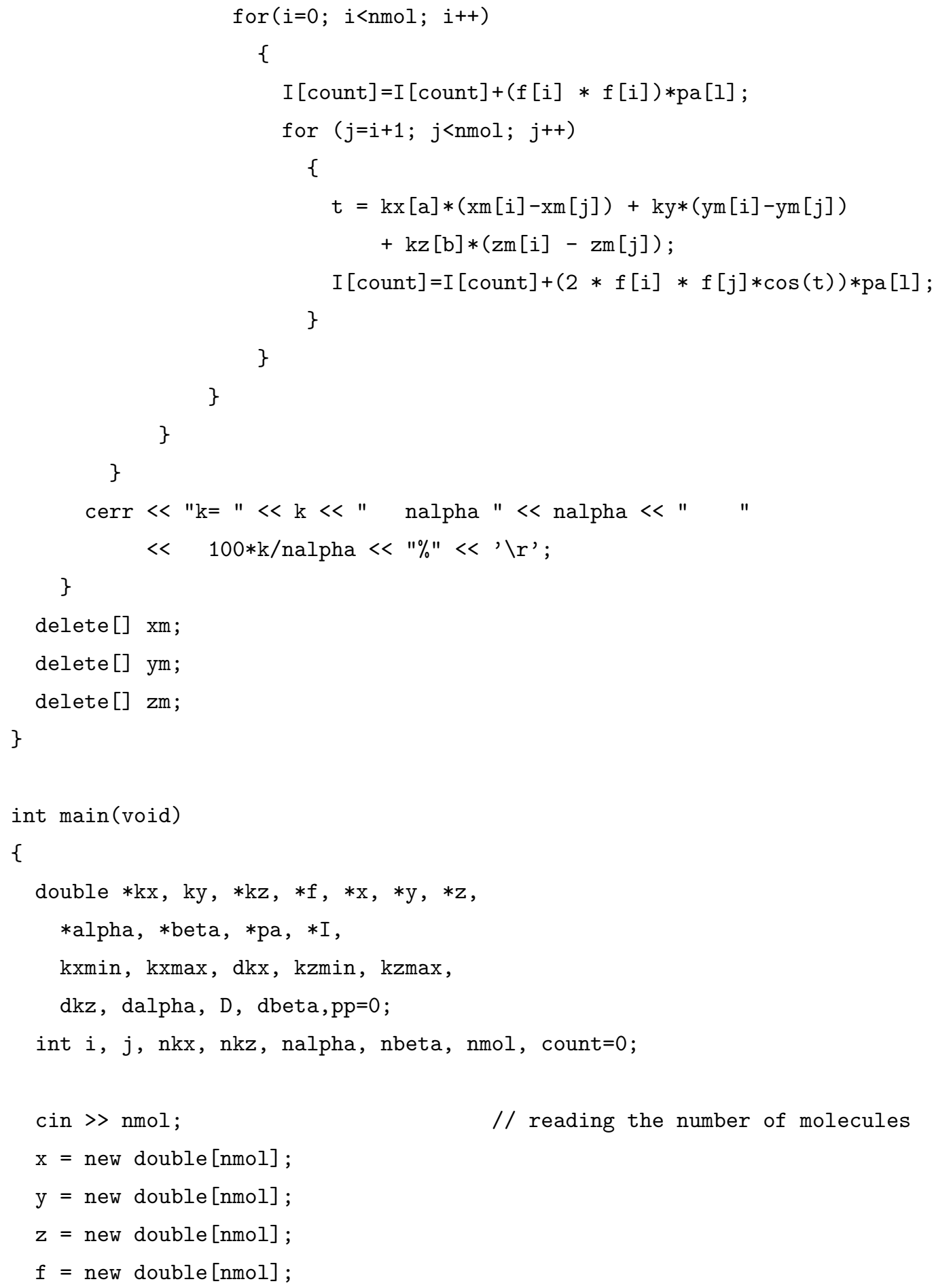




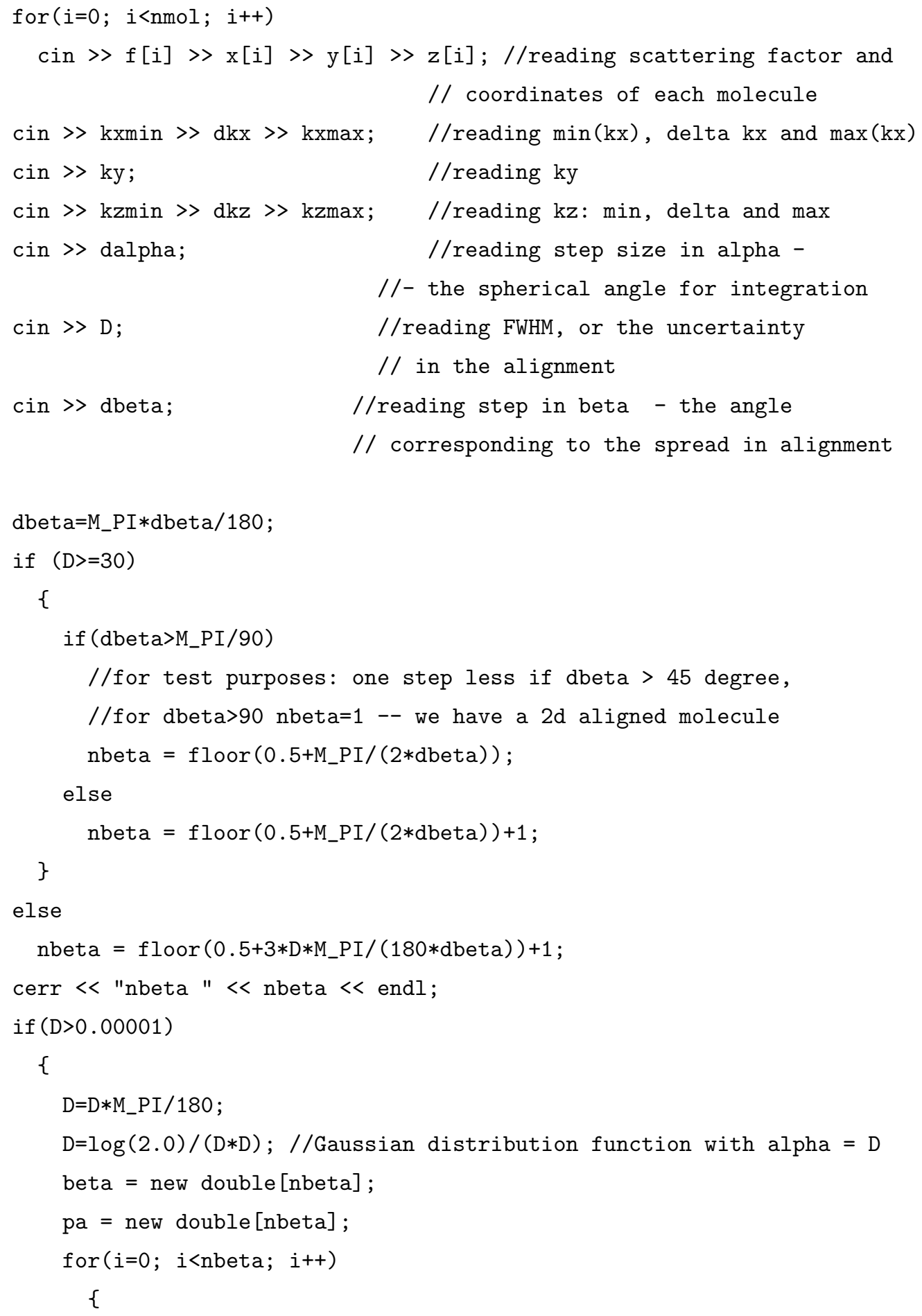




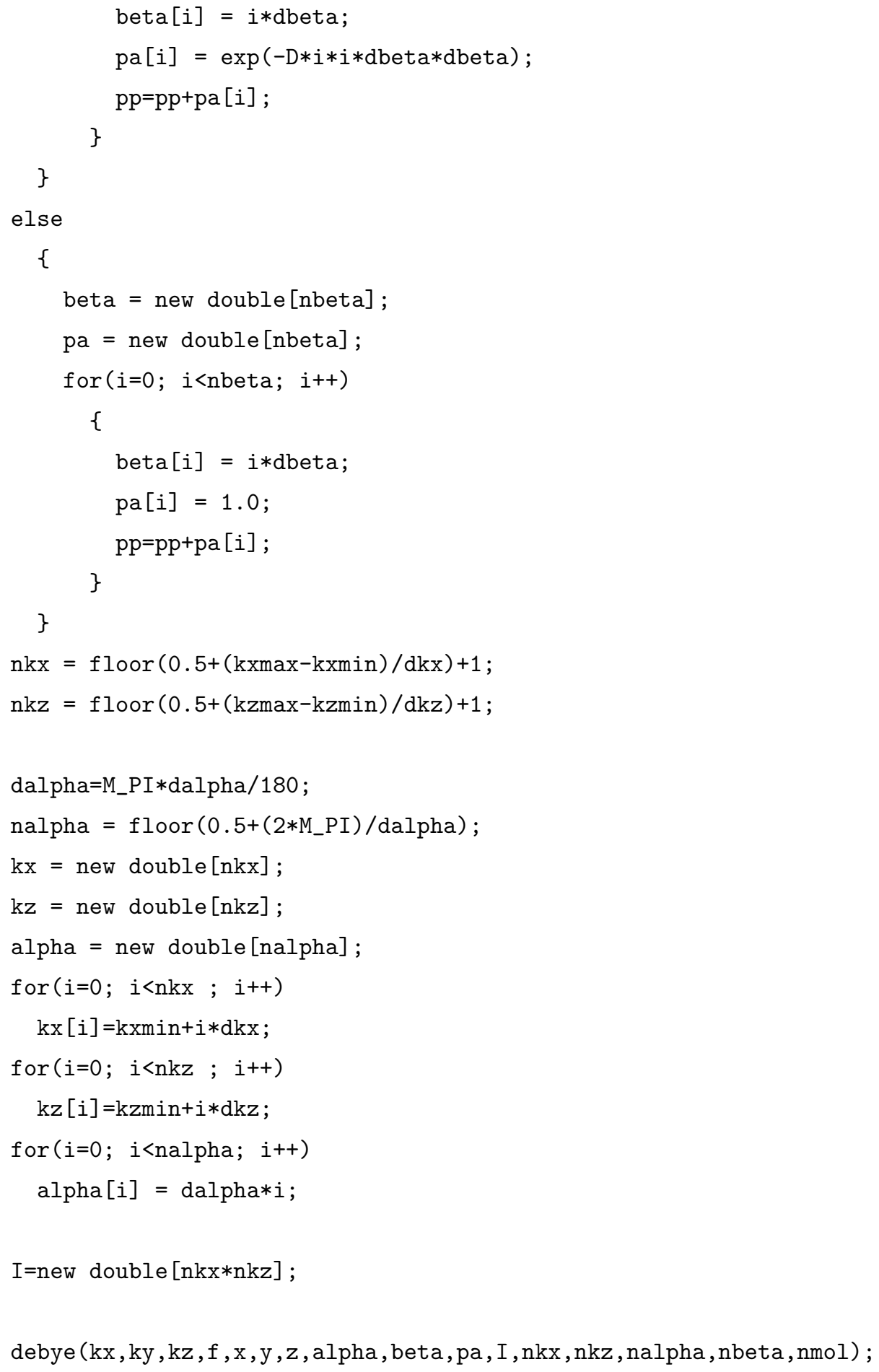




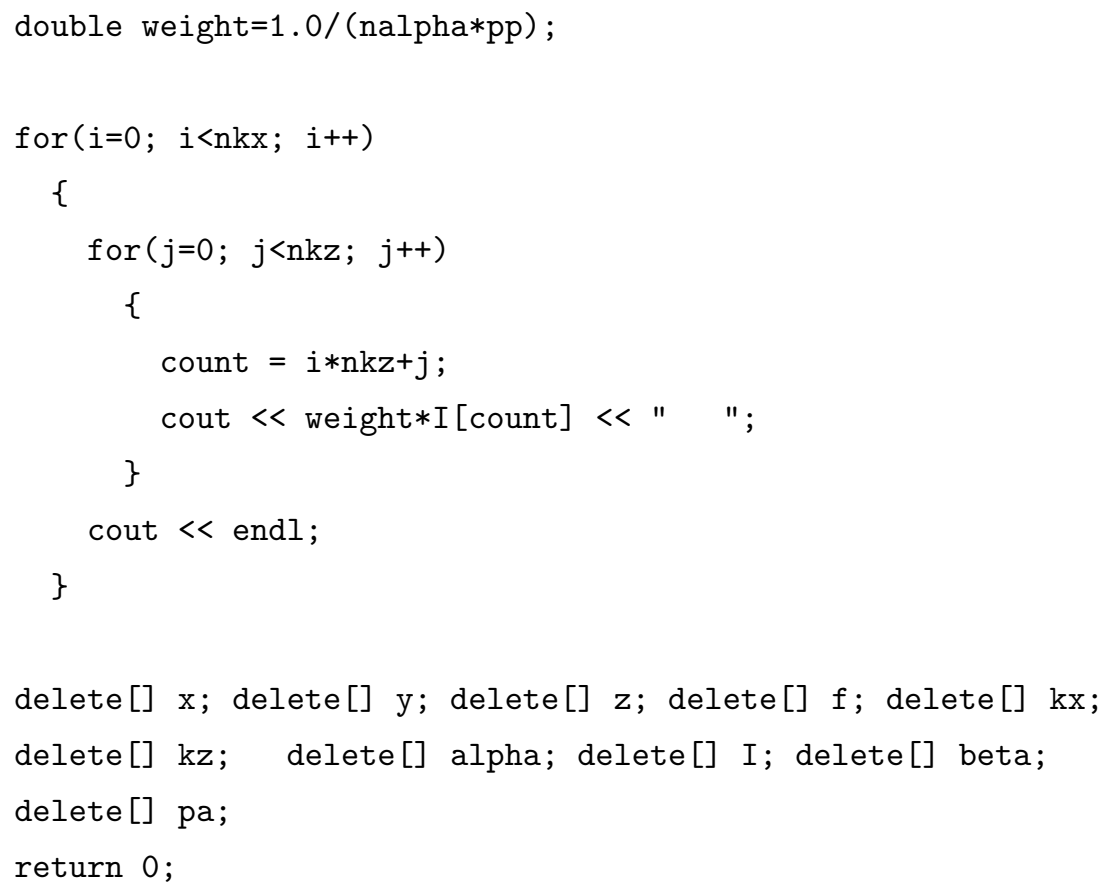




\title{
Lebenslauf von Andrea Debnárová
}

\author{
Geburtsdatum/-ort: 14 Oktober, 1979, Banská Bystrica, Slowakei \\ Nationalität: Slowakisch
}

\author{
Ausbildung \\ 10/2006-09/2009 Promotionsstudium-Physik \\ Max-Planck-Institut für biophysikalische Chemie, Göttingen \\ und Georg-August Universität, Göttingen \\ Betreuer: $\quad$ Prof. Markus Münzenberg \\ Thema: "Ab-initio Studies of X-ray Scattering" \\ 09/1998-06/2003 Hauptstudium - Physik/Festkörperphysik \\ Comenius University, Bratislava, Slowakei \\ Betreuer: $\quad$ RNDr. Eva Majková, DrSc. \\ Thema: "X-ray Scattering Study of Sc/Cr Multilayers" \\ 1998 Abitur, Gymnasium in Rimavská Sobota, Slowakei
}

